\title{
Regiodivergent Oxidative Cross-Coupling of Catechols with Persistent tert-Carbon Radicals
}

Masumi Sugawara,$^{\dagger}$ Rikako Ohnishi, ${ }^{\dagger}$ Tetsuya Ezawa ${ }^{\ddagger}$ Mai Akakabe, ${ }^{\dagger}$ Miki Sawamura ${ }^{\dagger}$ Daiki Hojo, ${ }^{\ddagger}$ Daisuke Hashizume, ${ }^{\S}$ Yoshihiro Sohtome, ${ }^{* \dagger, *}$ and Mikiko Sodeoka ${ }^{*,+, \$}$

${ }^{\dagger}$ Synthetic Organic Chemistry Laboratory, RIKEN Cluster for Pioneering Research, 2-1 Hirosawa, Wako, Saitama, 3510198 Japan

${ }^{\dagger}$ RIKEN Center for Sustainable Resource Science, 351-0198, 2-1 Hirosawa, Wako, Saitama, 351-0198 Japan

${ }^{9}$ Graduate School of Science and Engineering, Saitama University; 255 Shimo-okubo, Sakura-ku, Saitama, 338-8570 Japan

${ }^{\S}$ RIKEN Center for Emergent Matter Science, 2-1 Hirosawa, Wako, Saitama 351-0198 Japan

*E-Mail for Y. Sohtome: sohtome@riken.jp

*E-Mail for M. Sodeoka: sodeoka@ riken.jp 


\section{Supporting Information}

Table of Contents:

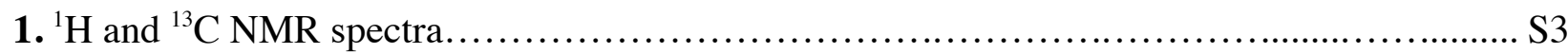

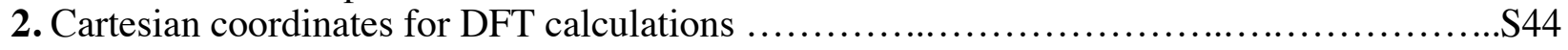




\section{1. ${ }^{1} \mathrm{H}$ and ${ }^{13} \mathrm{C}$ NMR spectra}
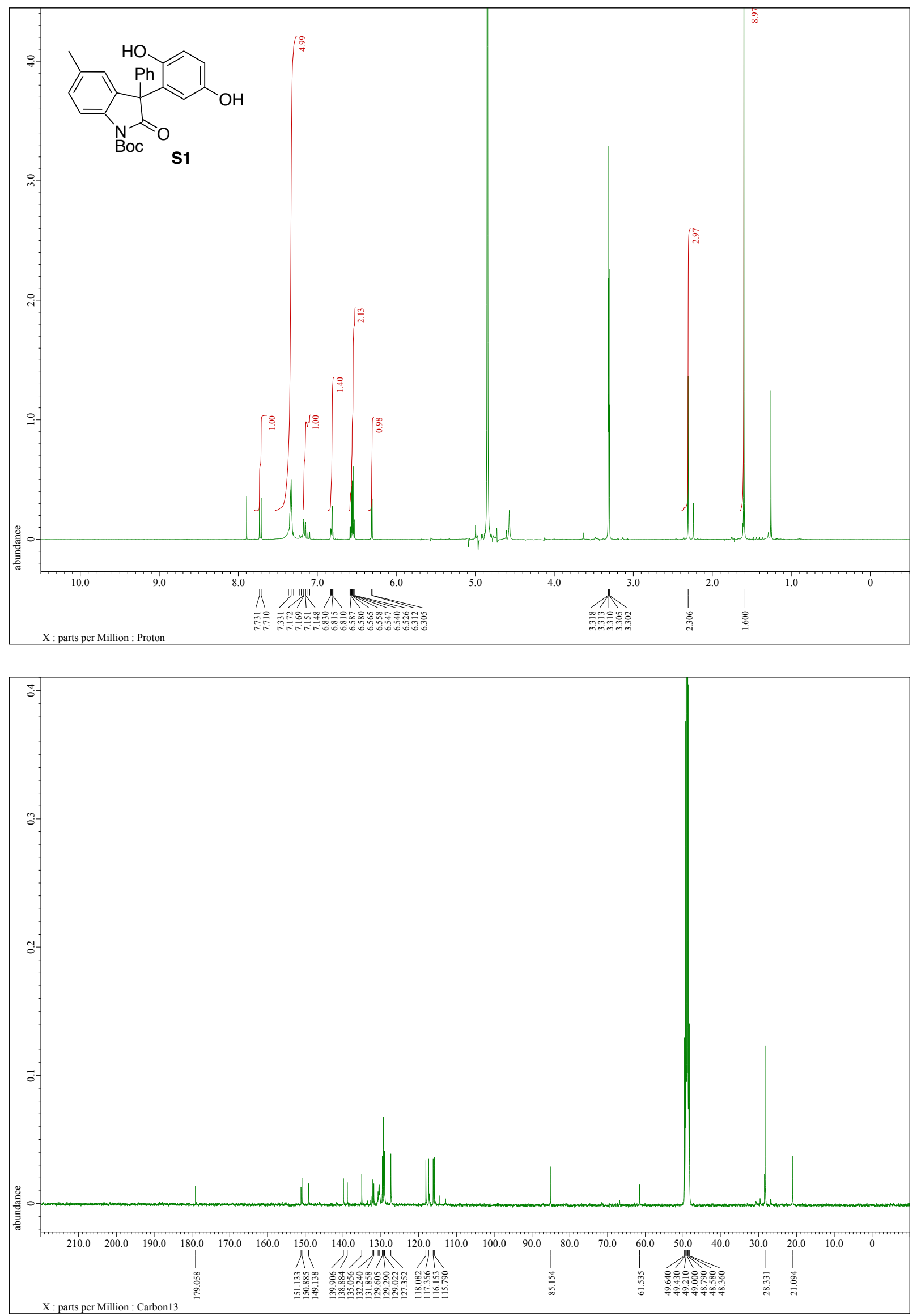

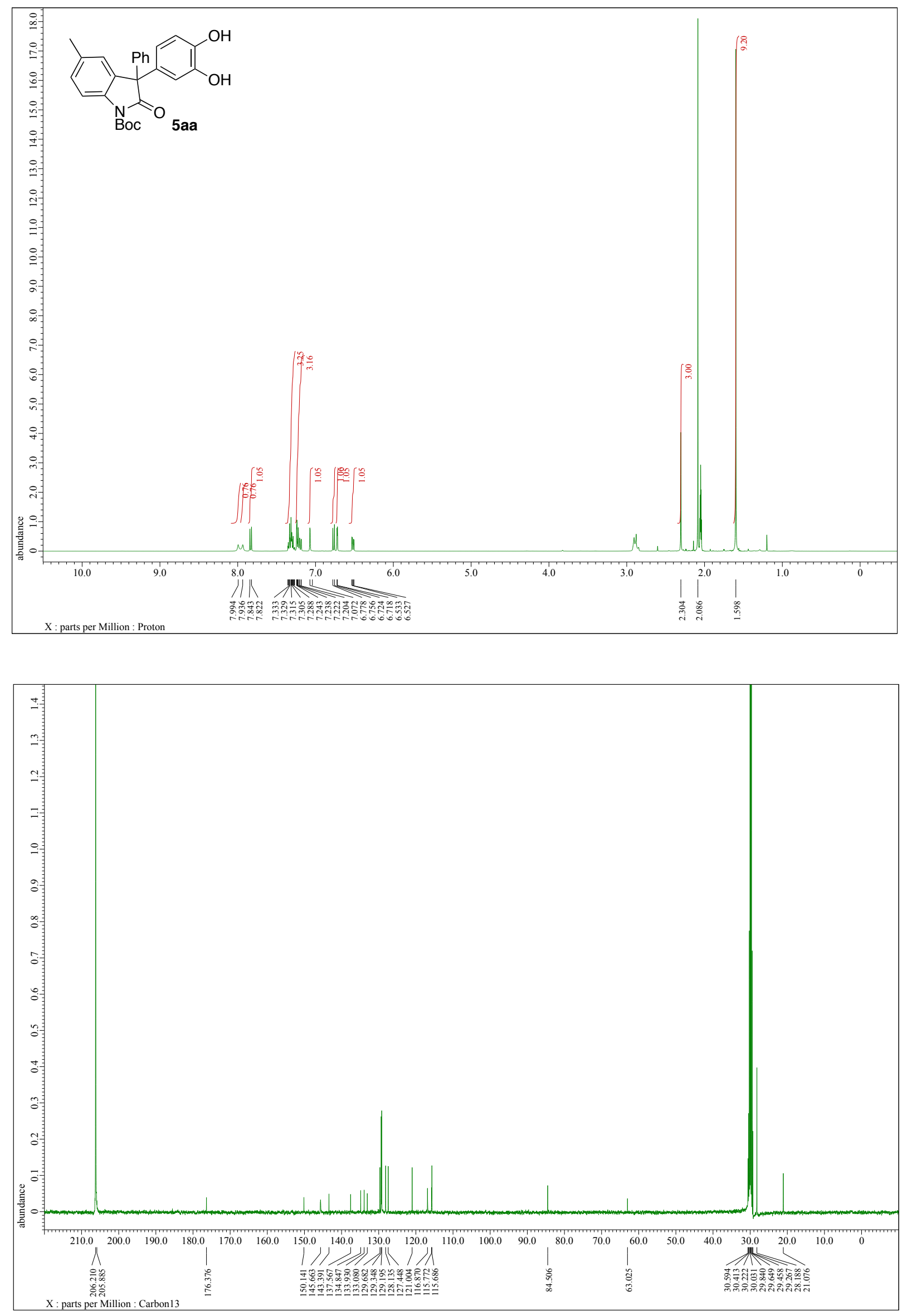

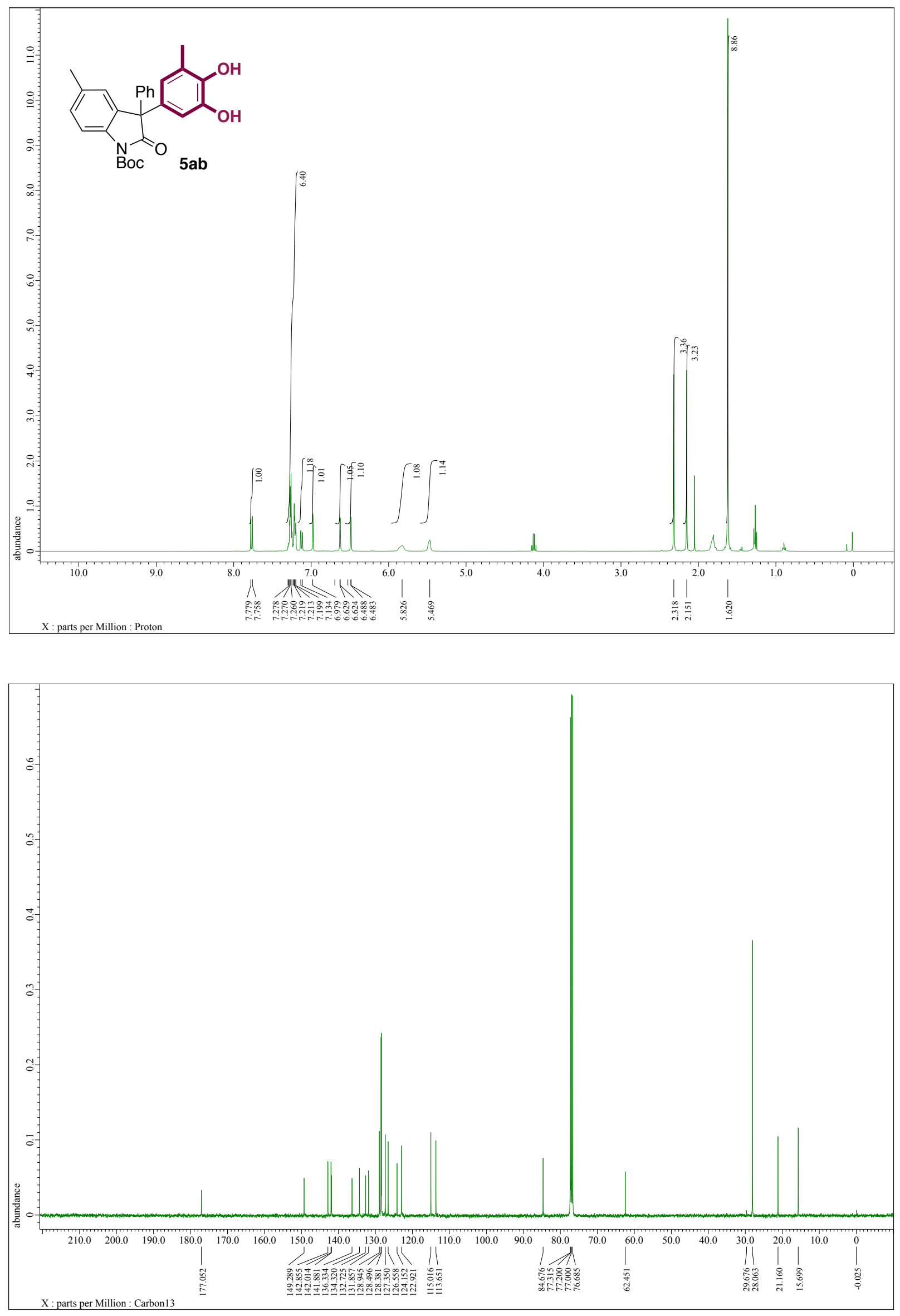

S5 

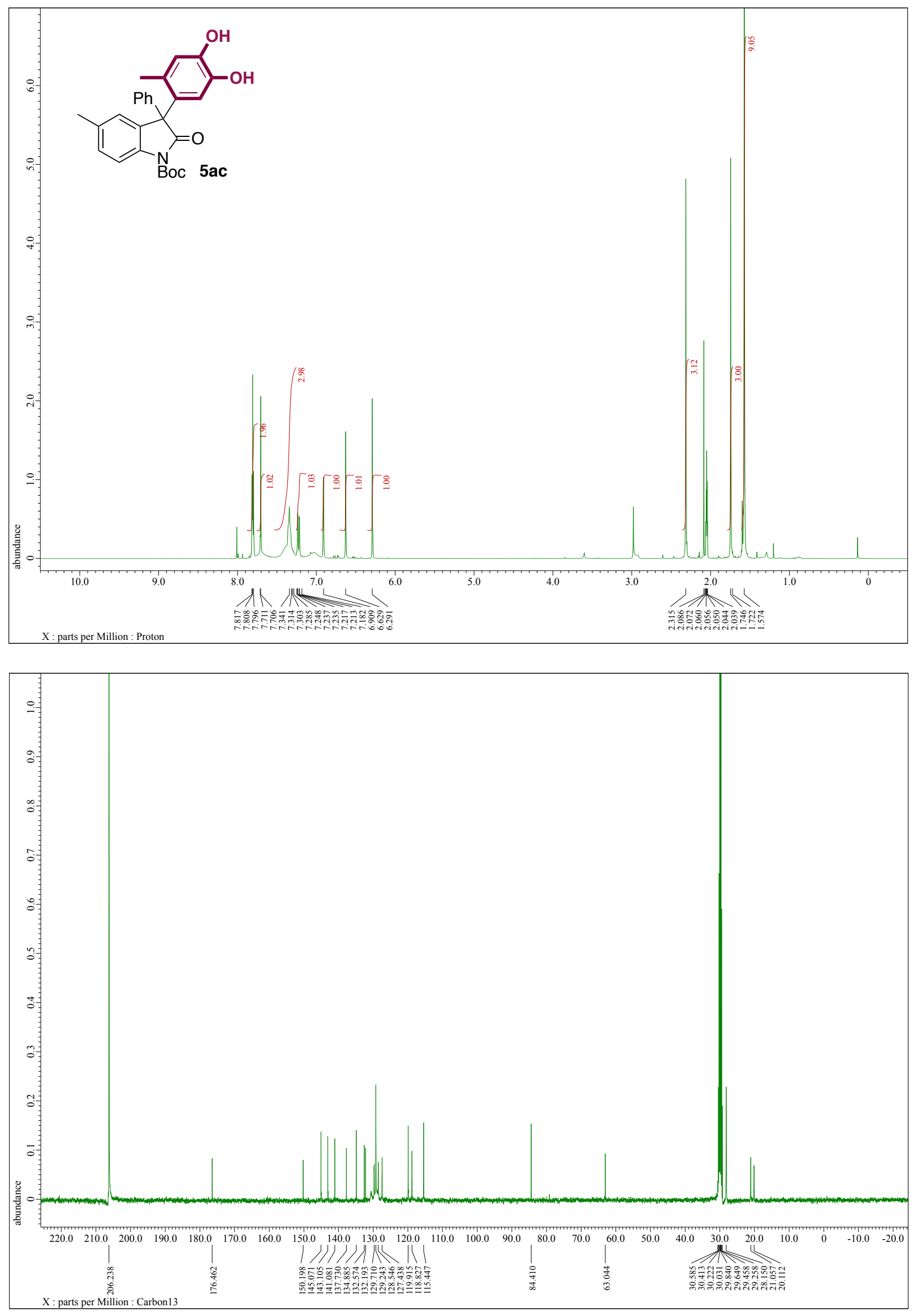

S6 

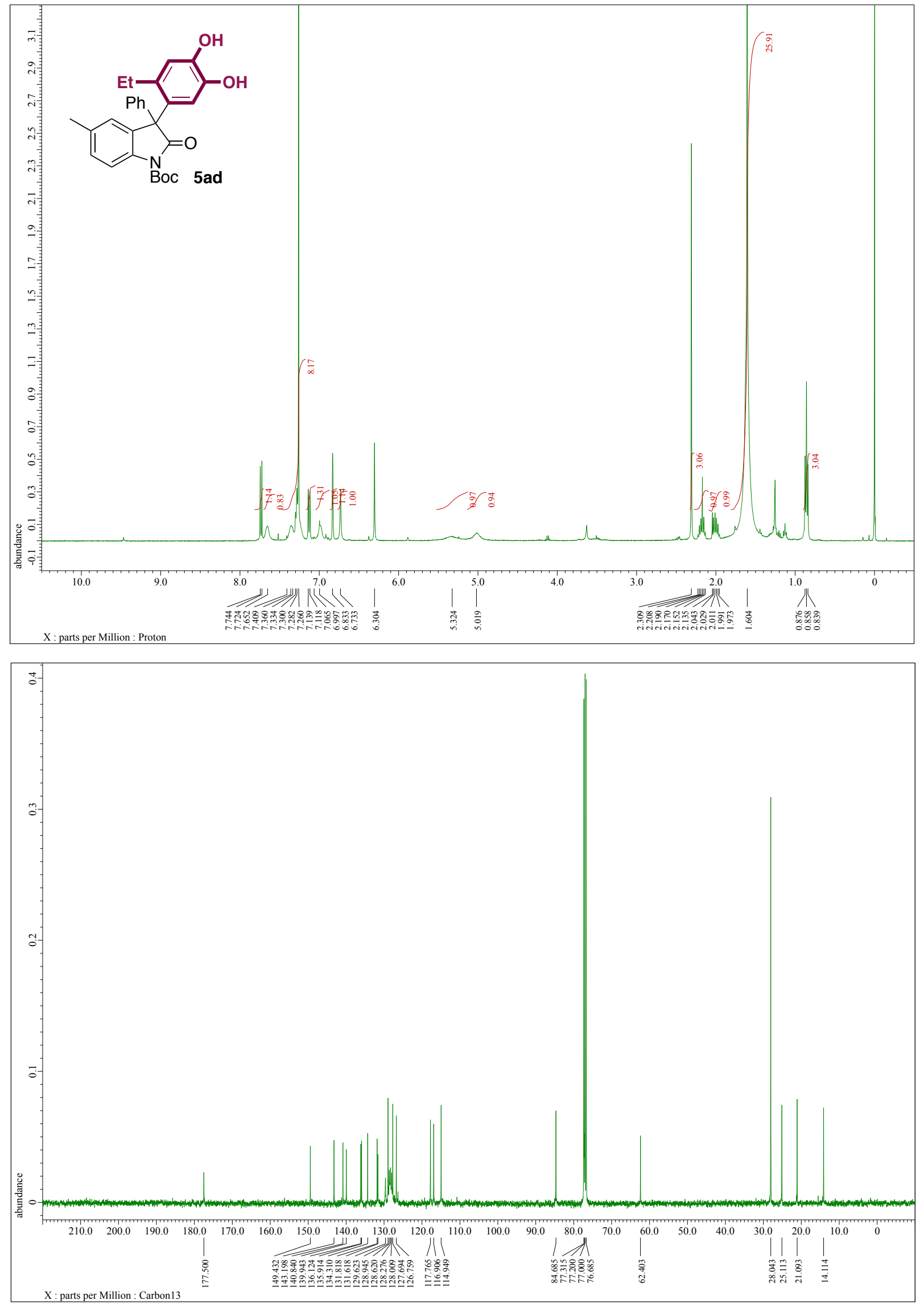

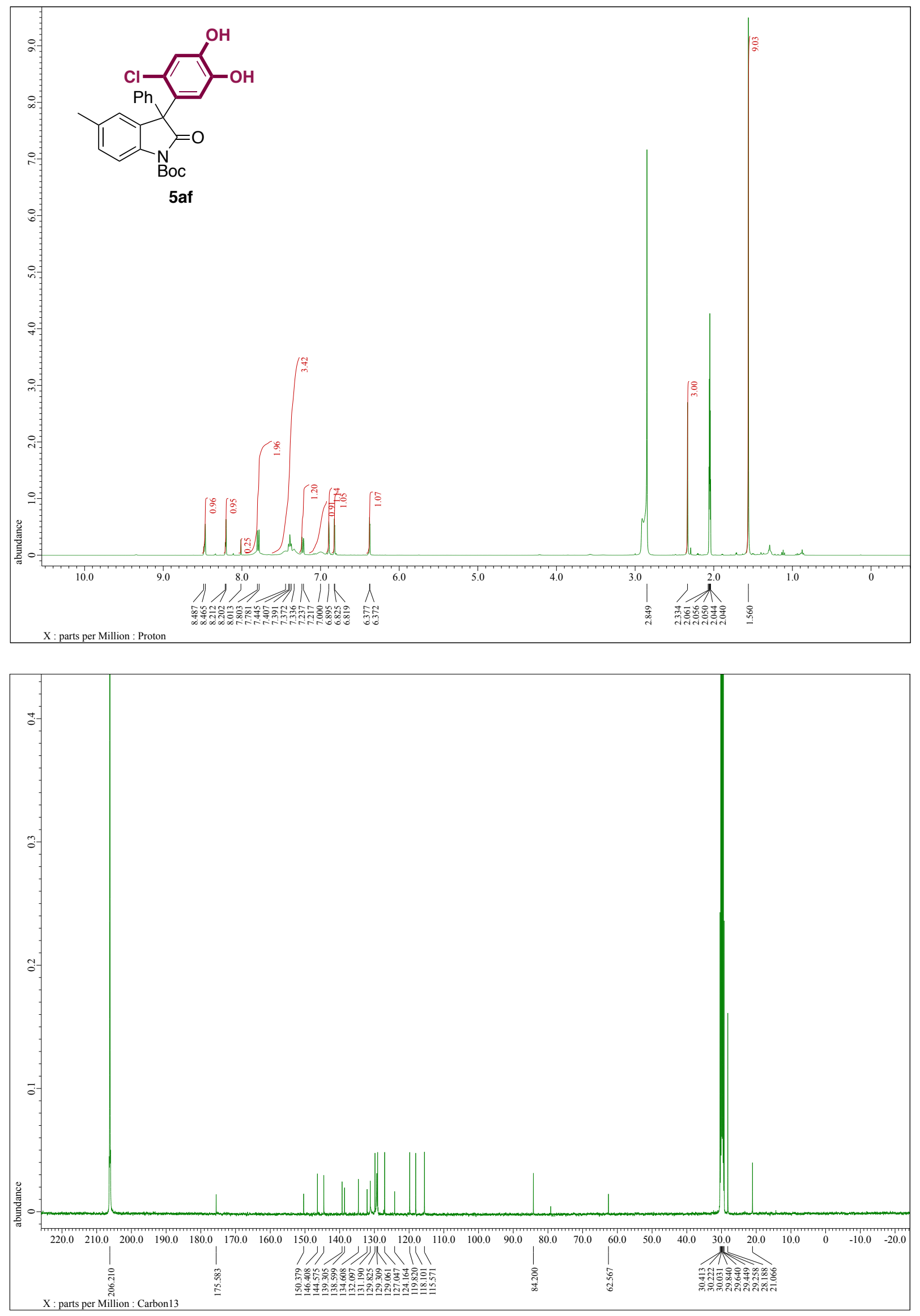

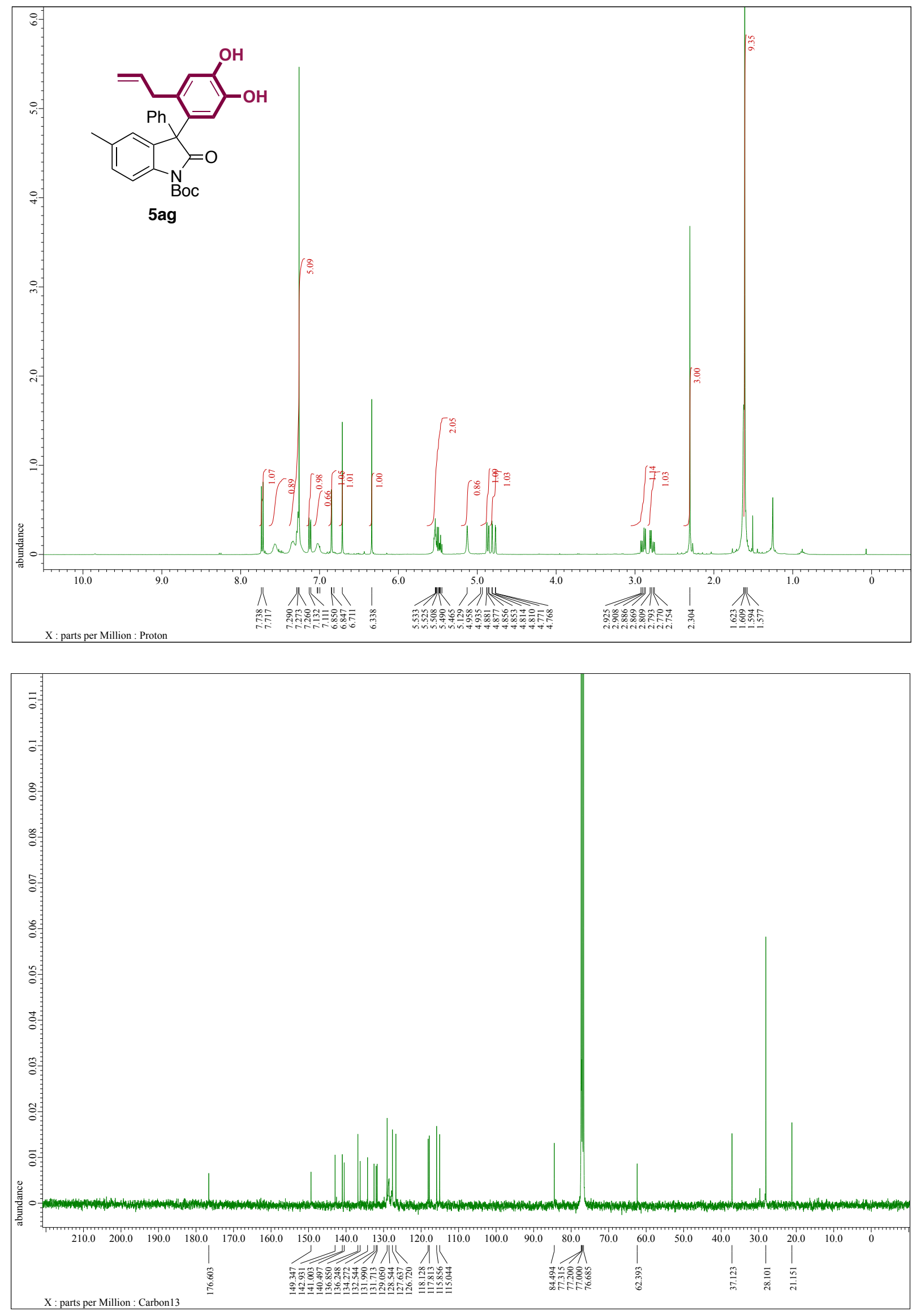

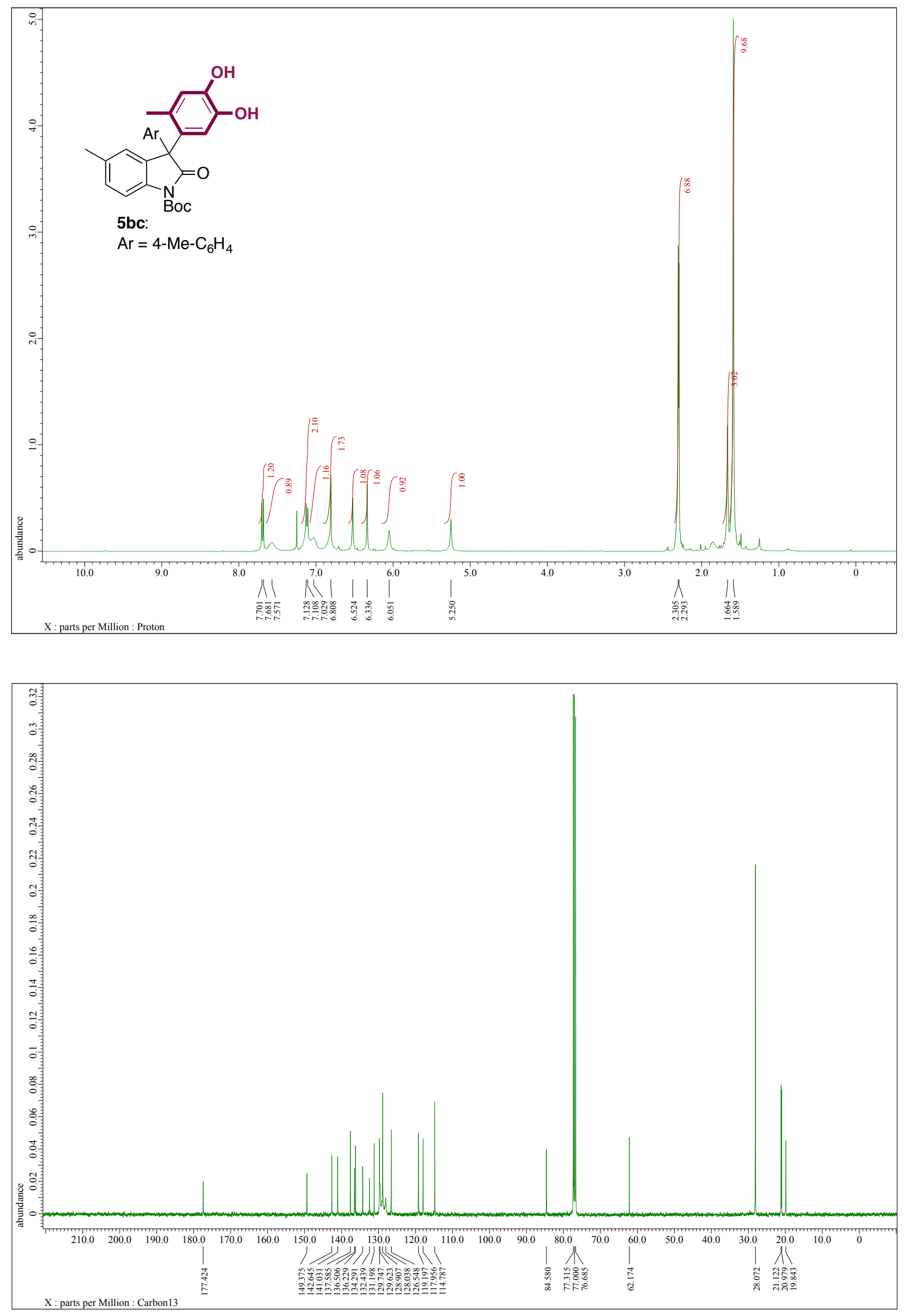

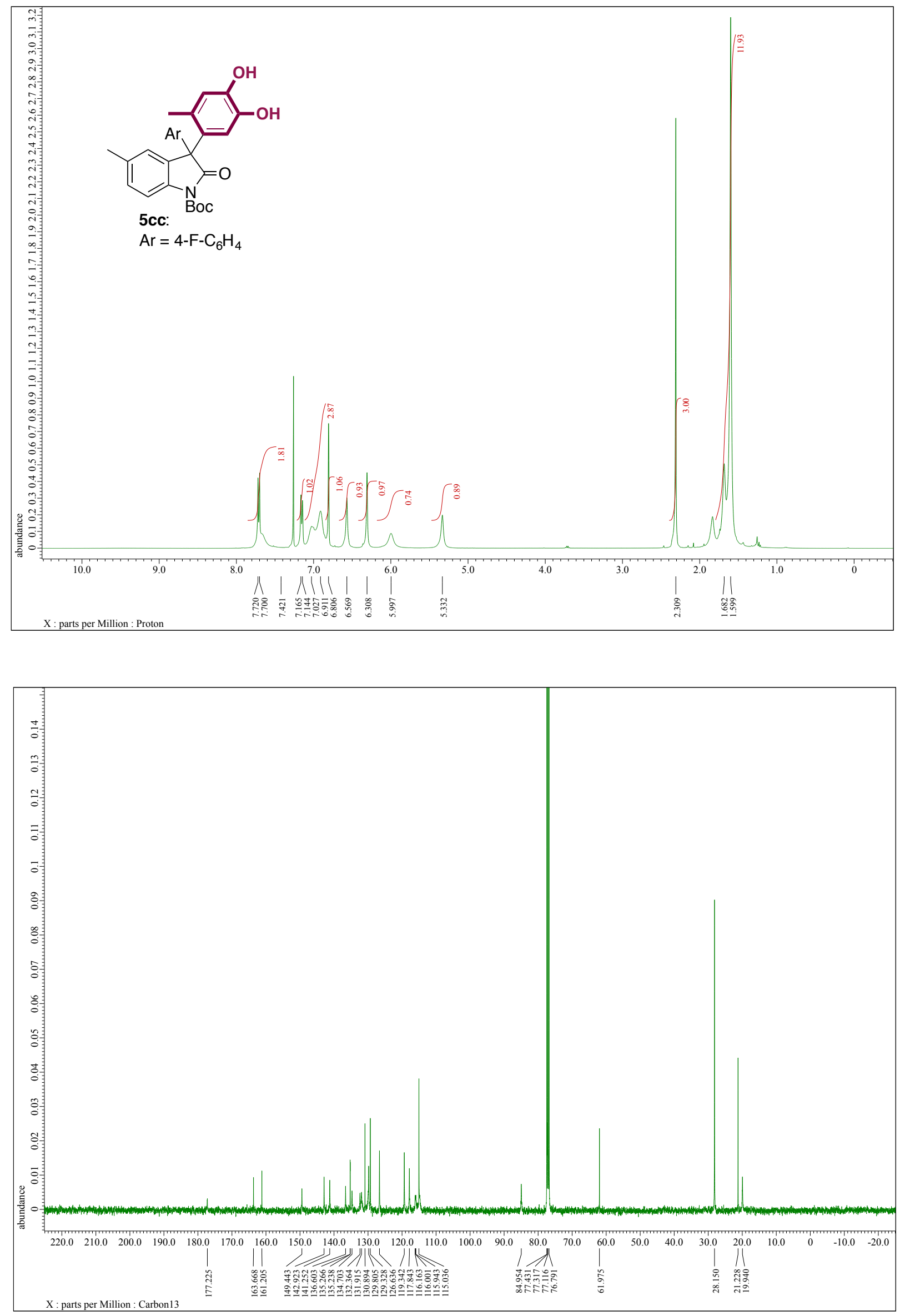

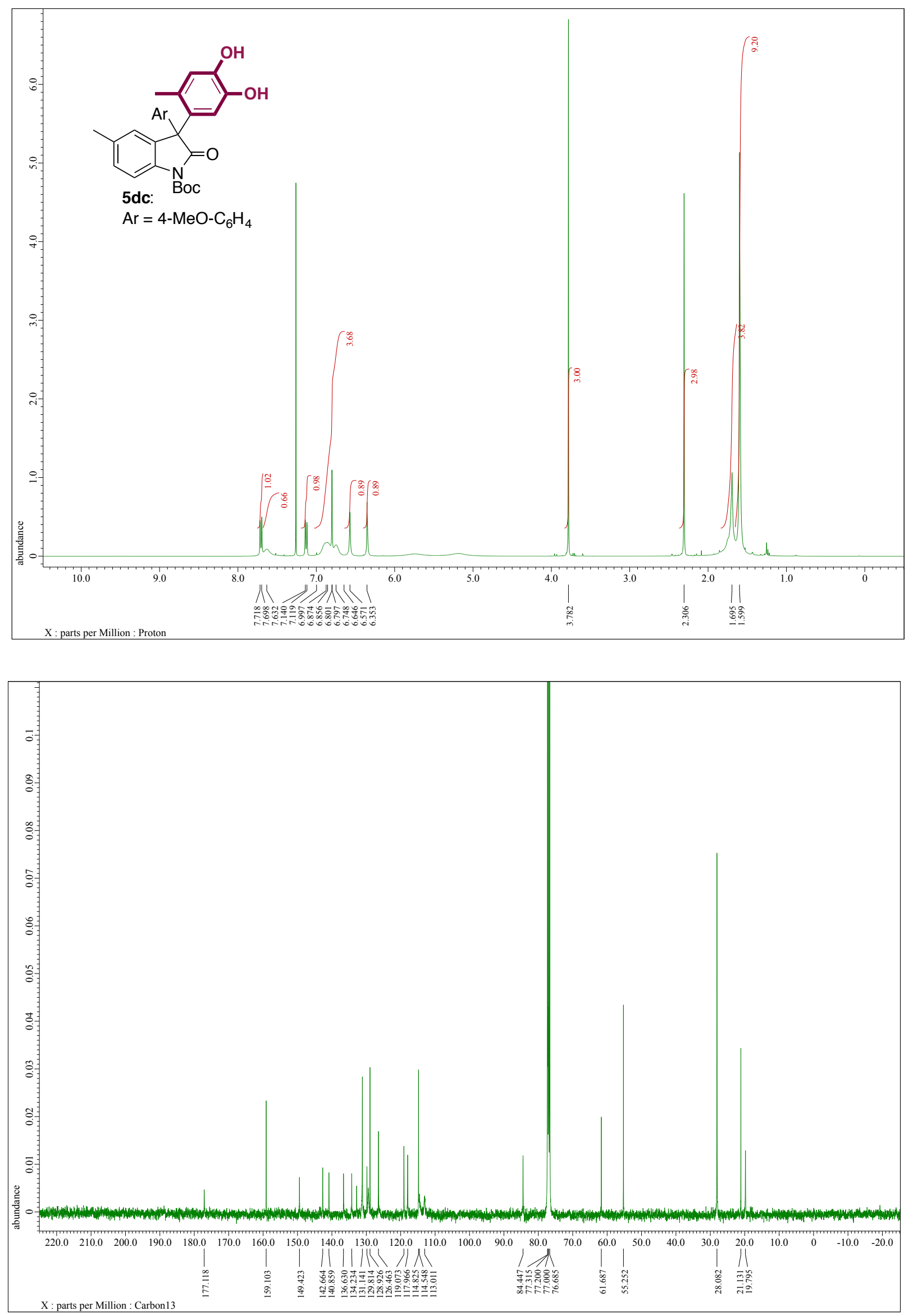

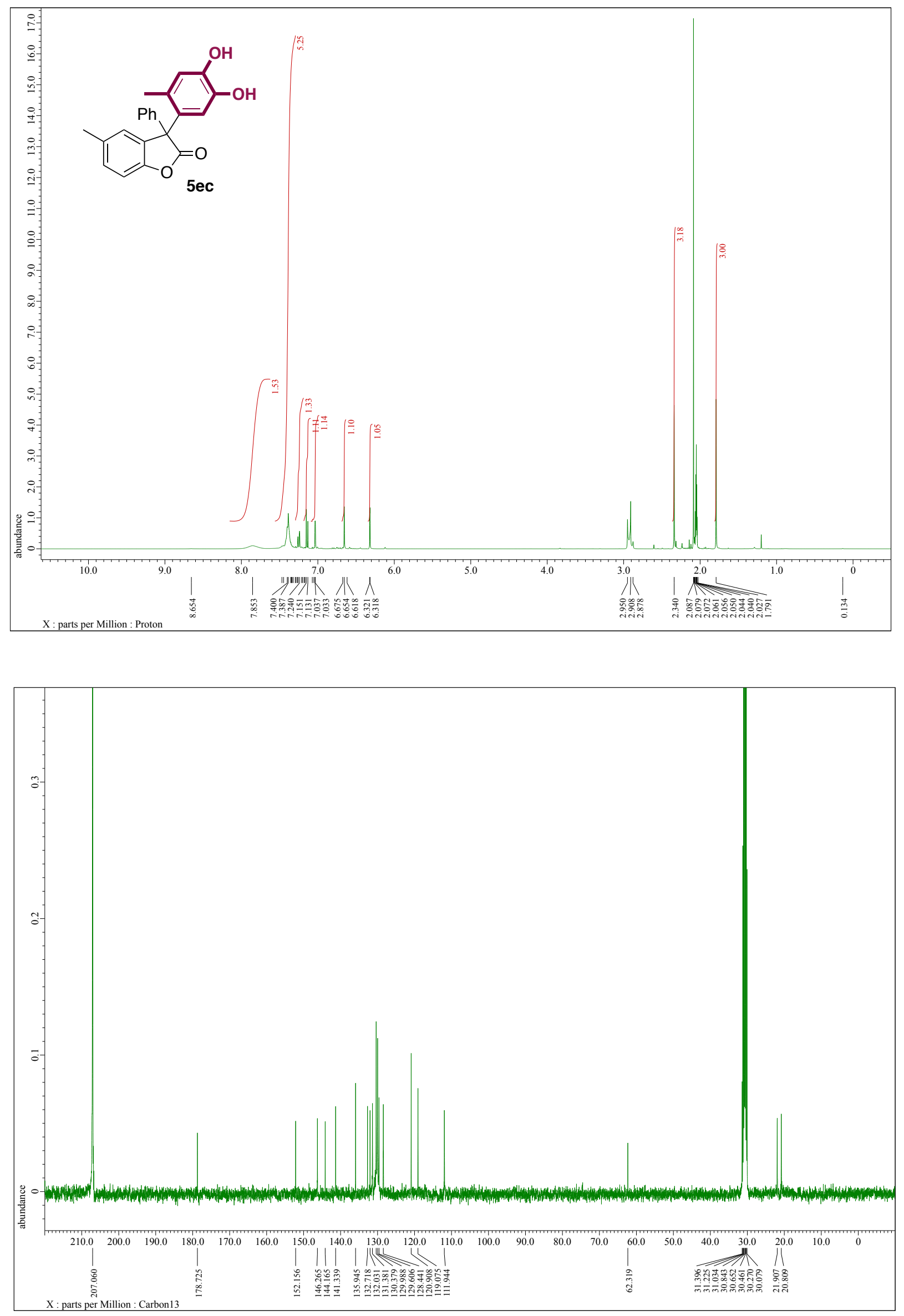

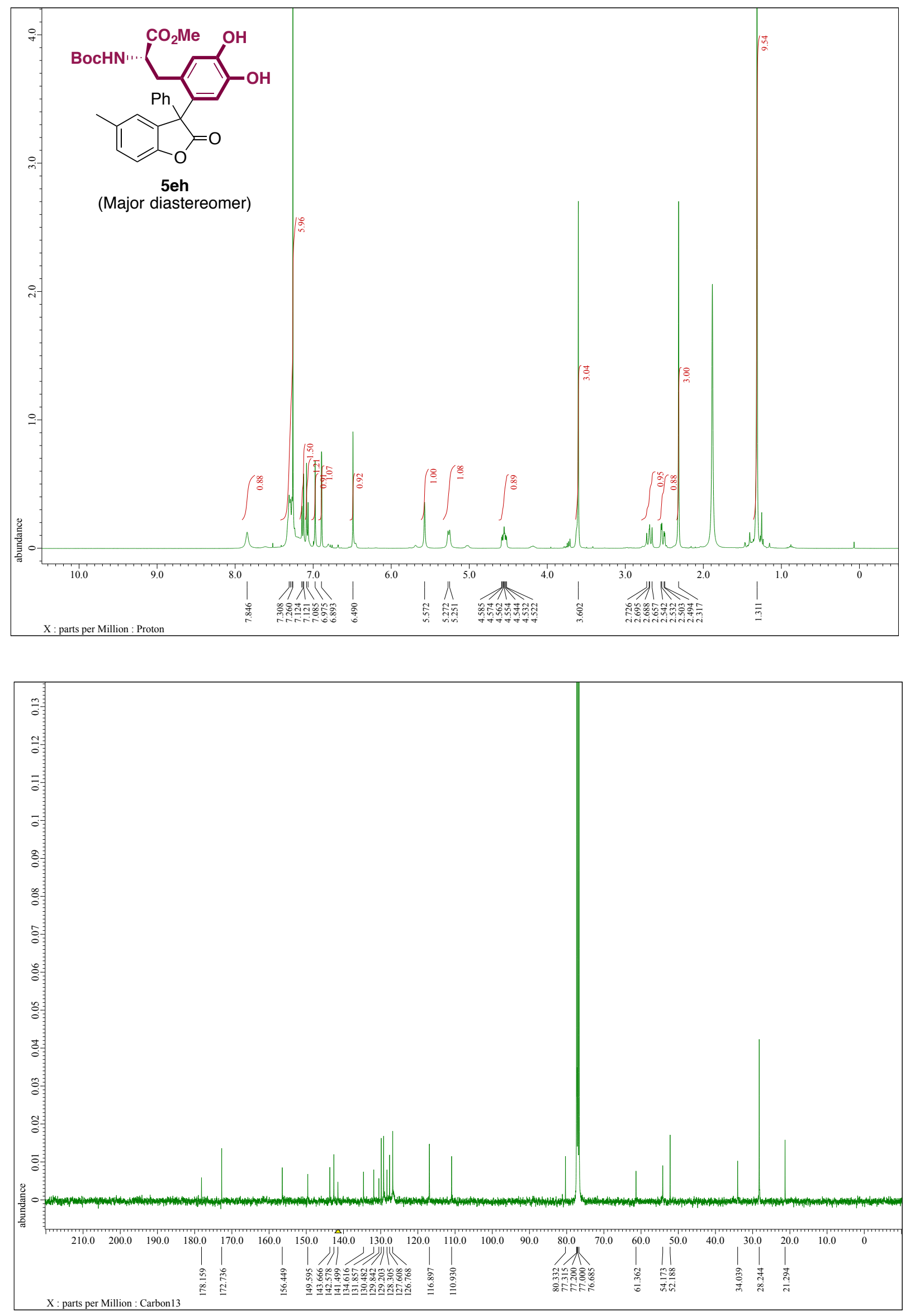

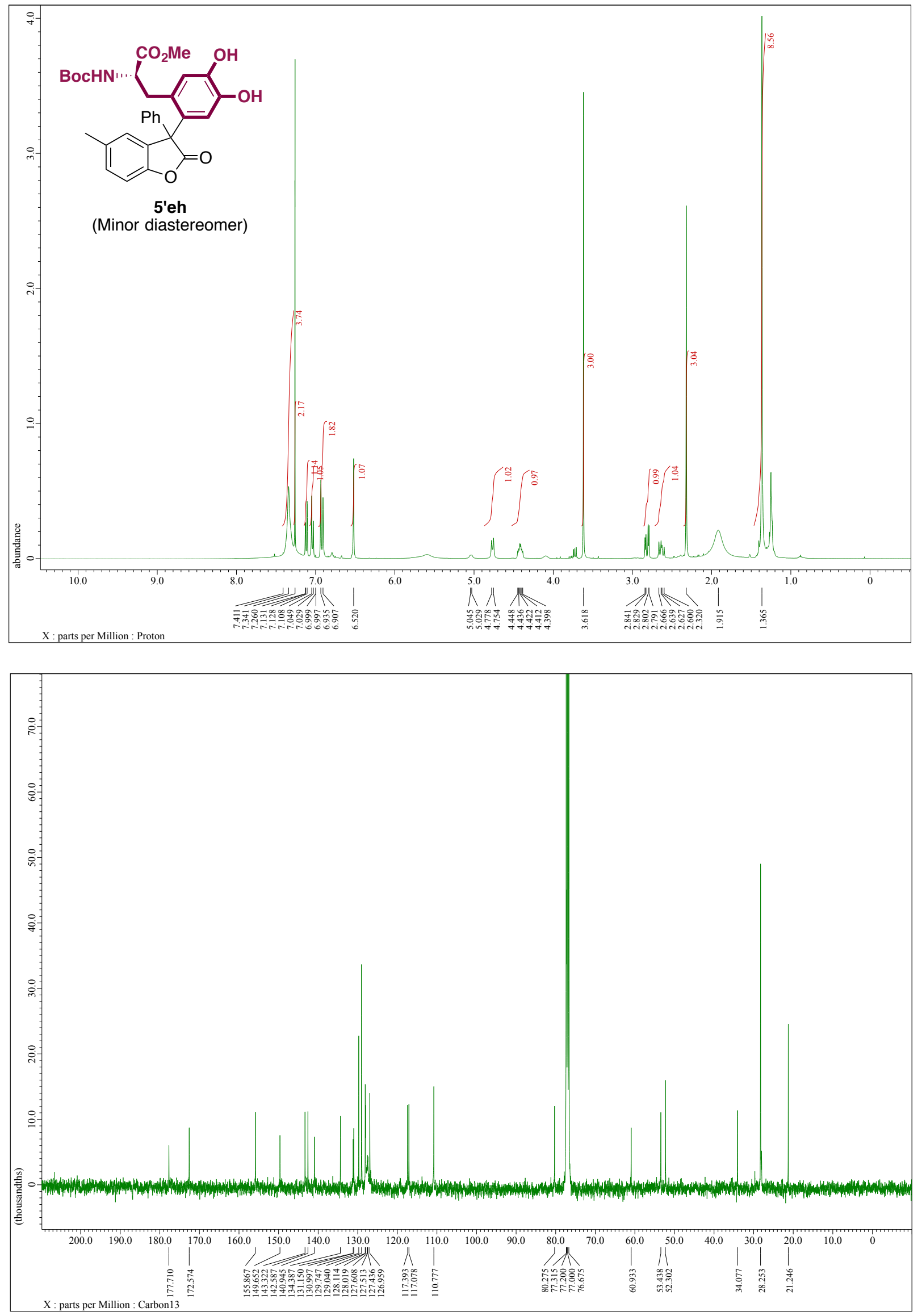

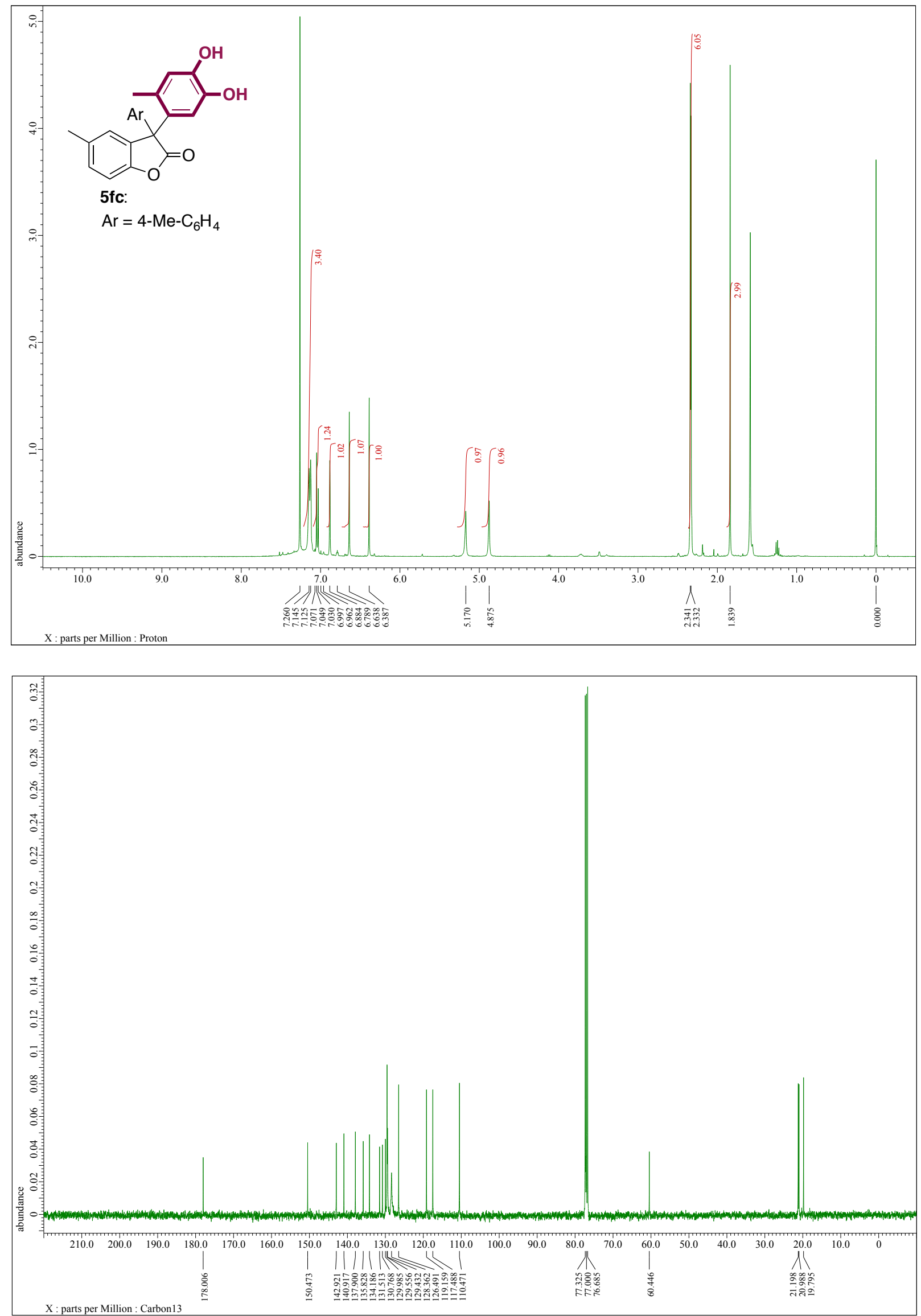

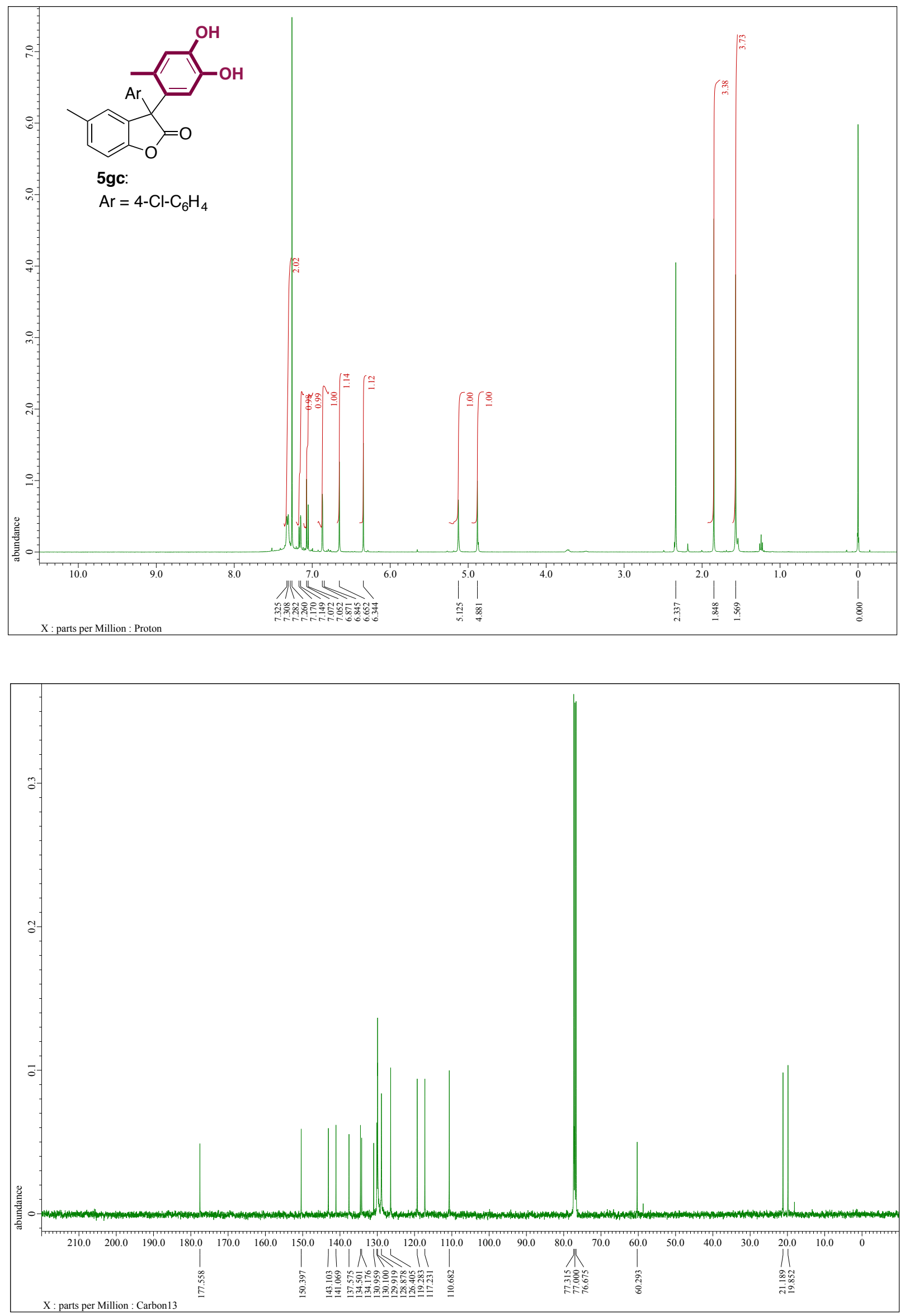

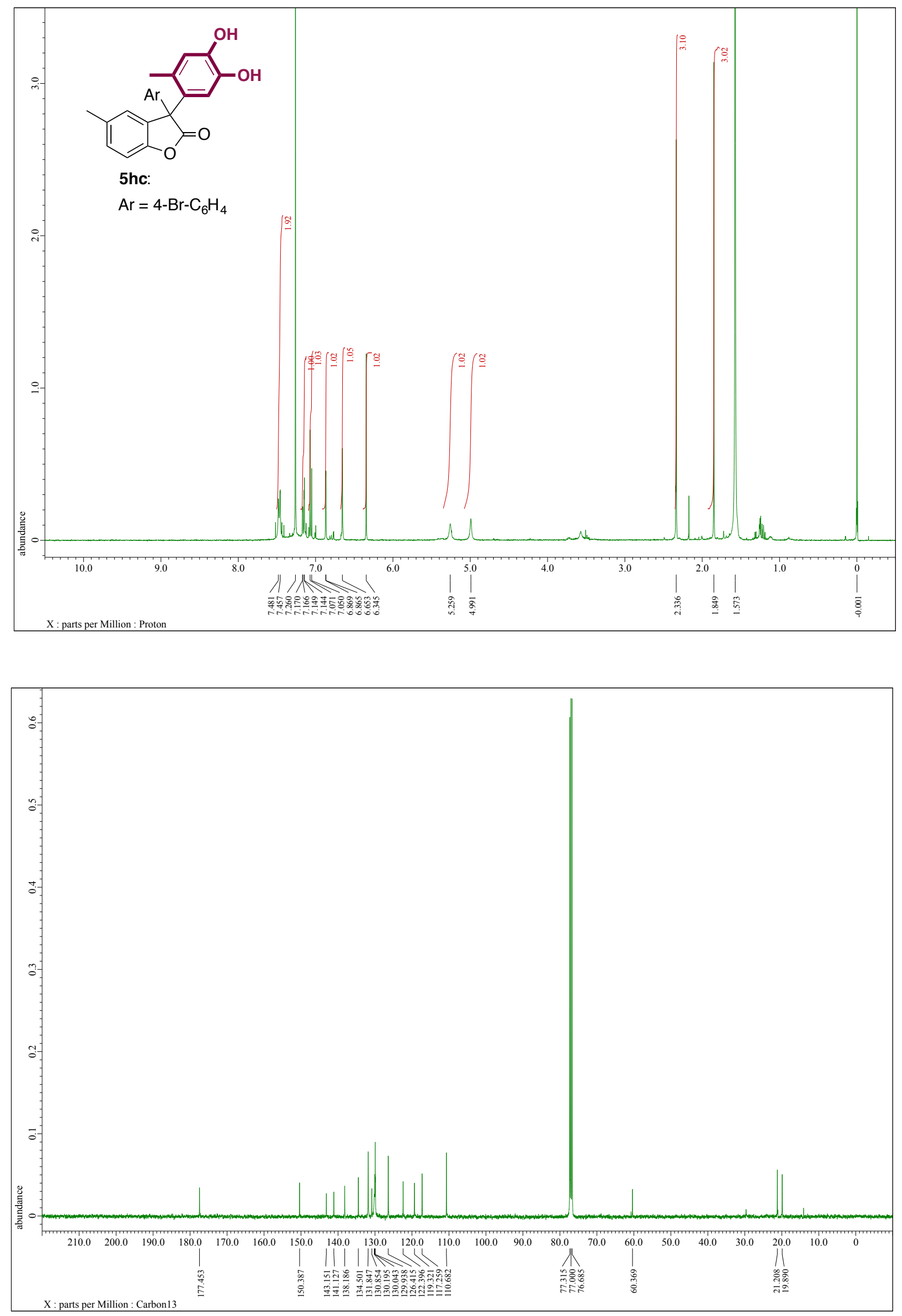

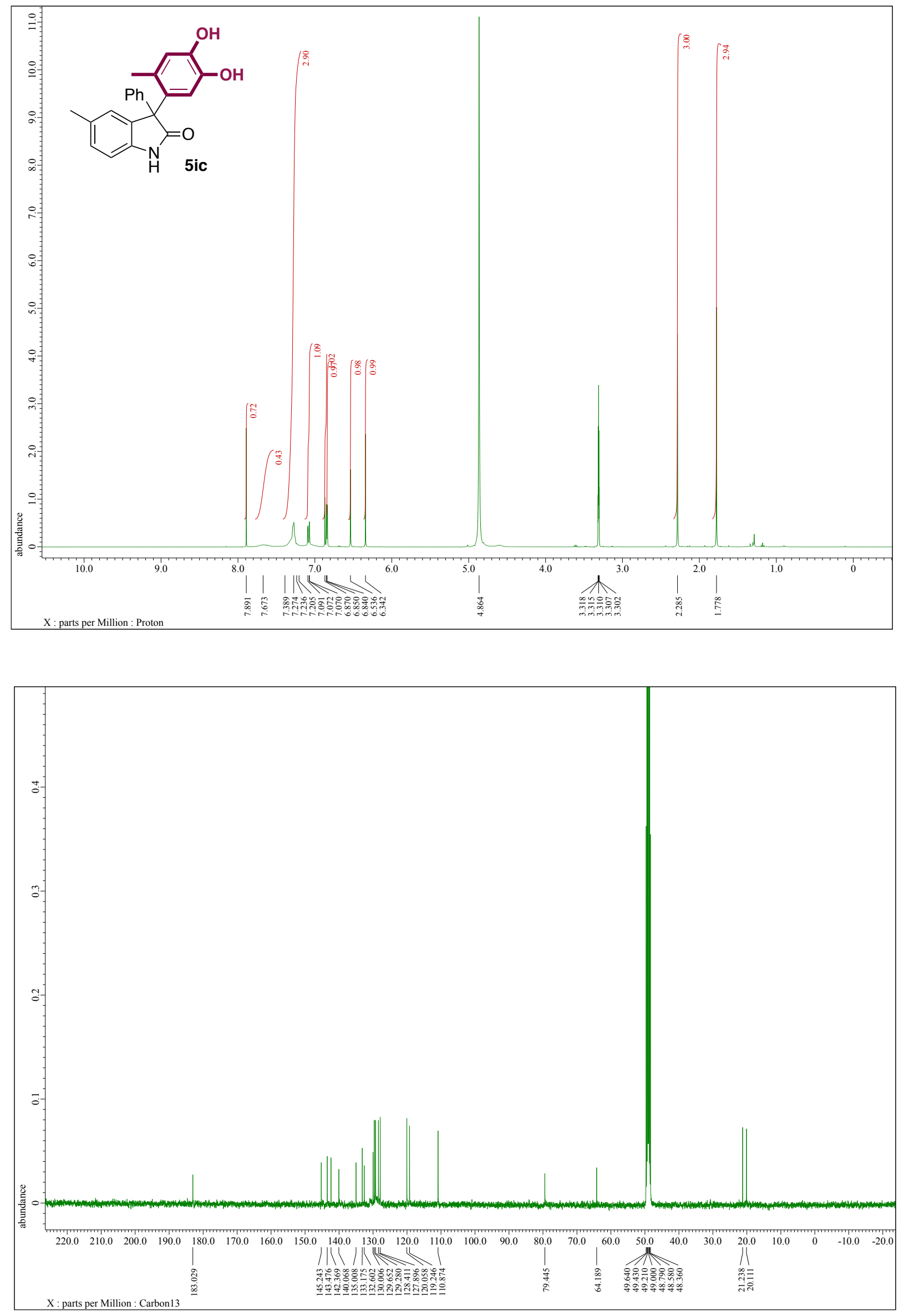

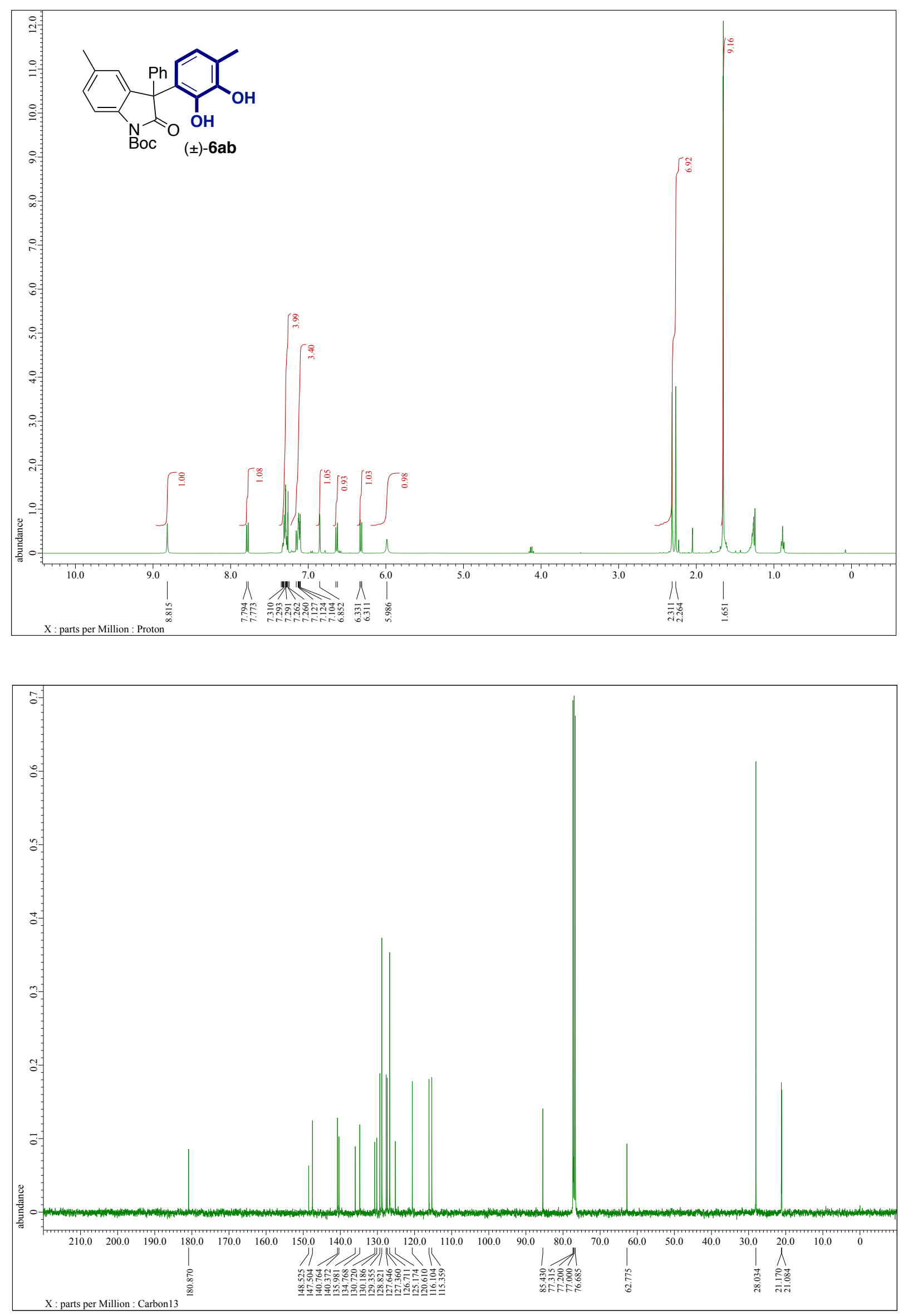

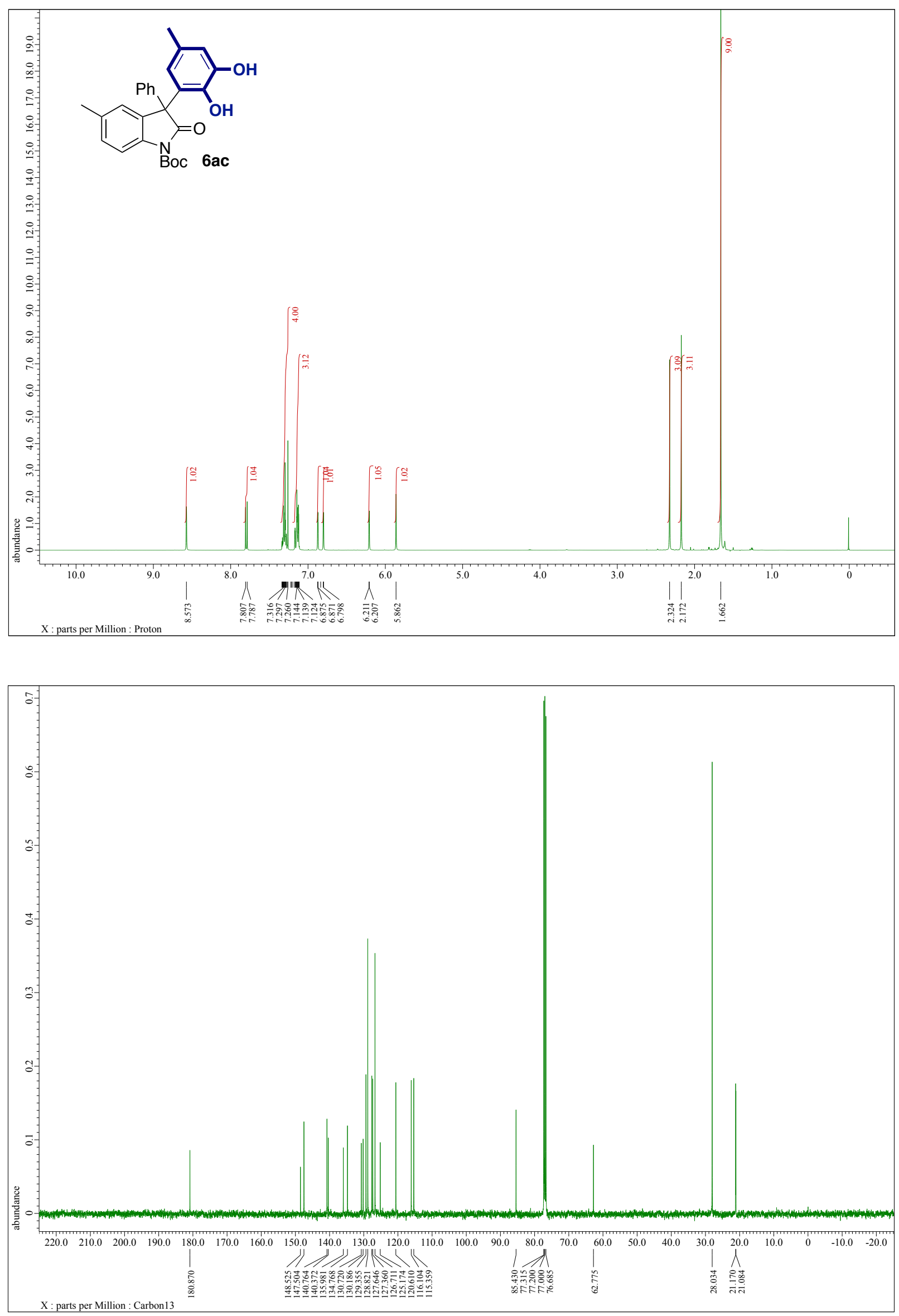

S21 

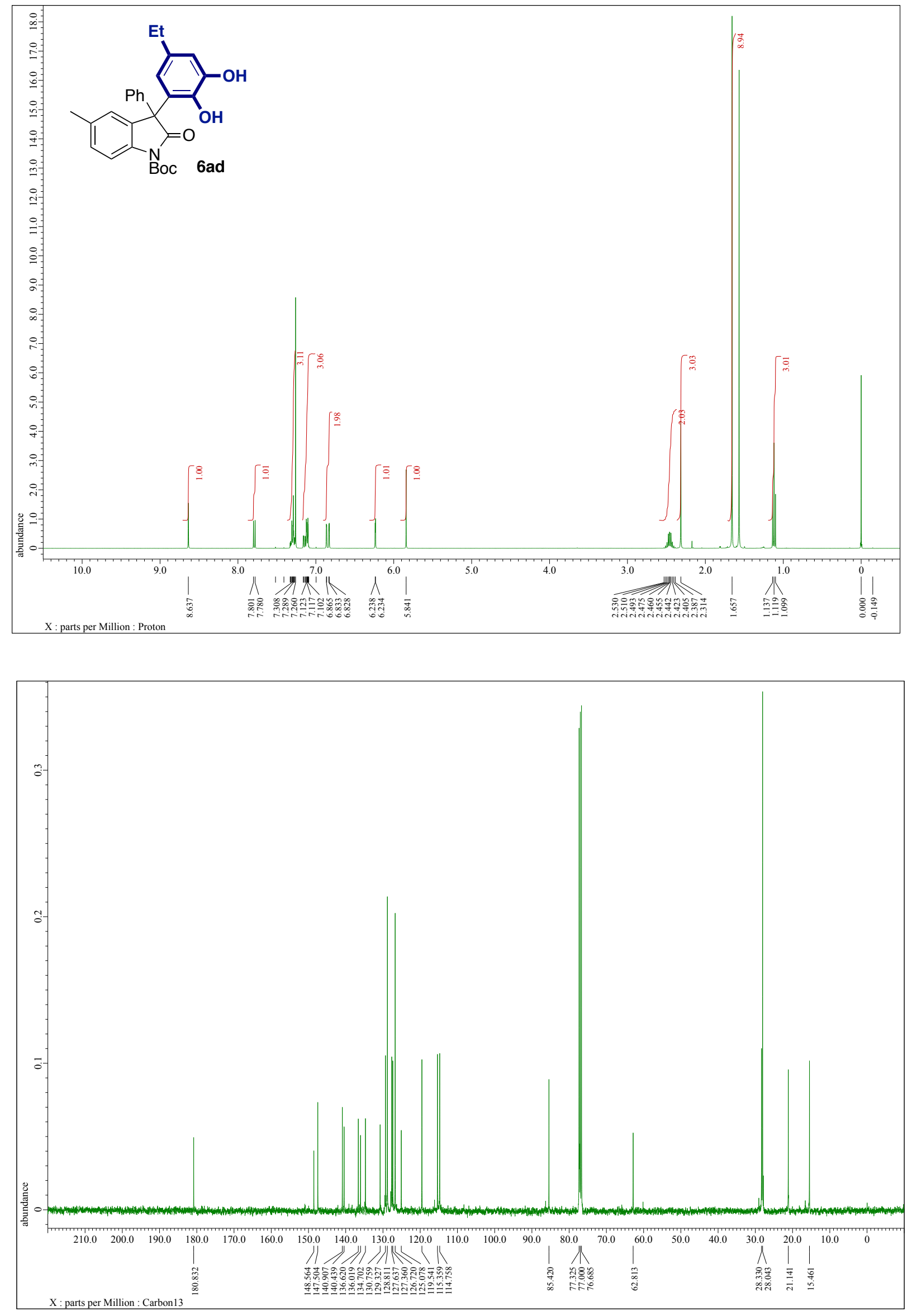

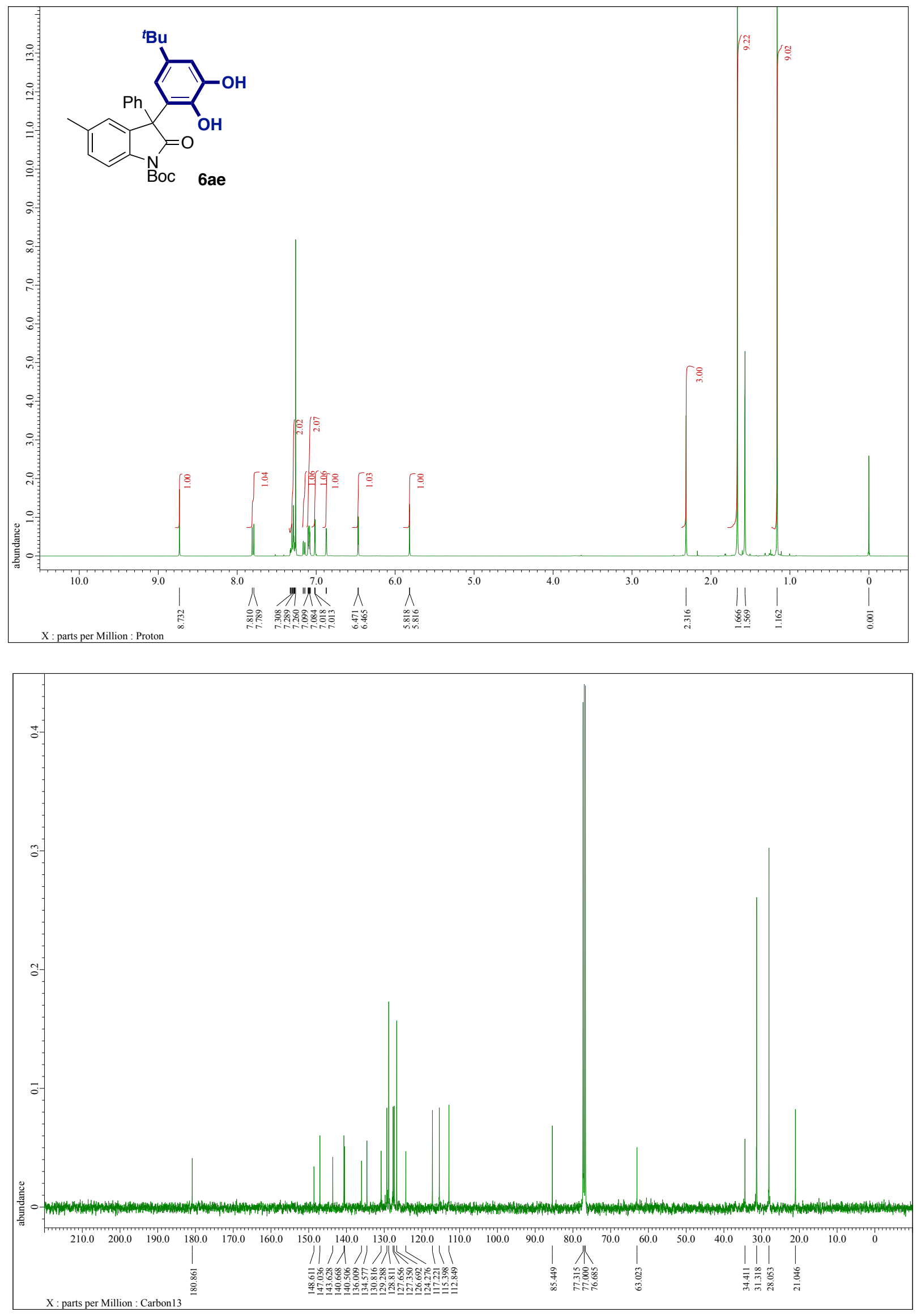

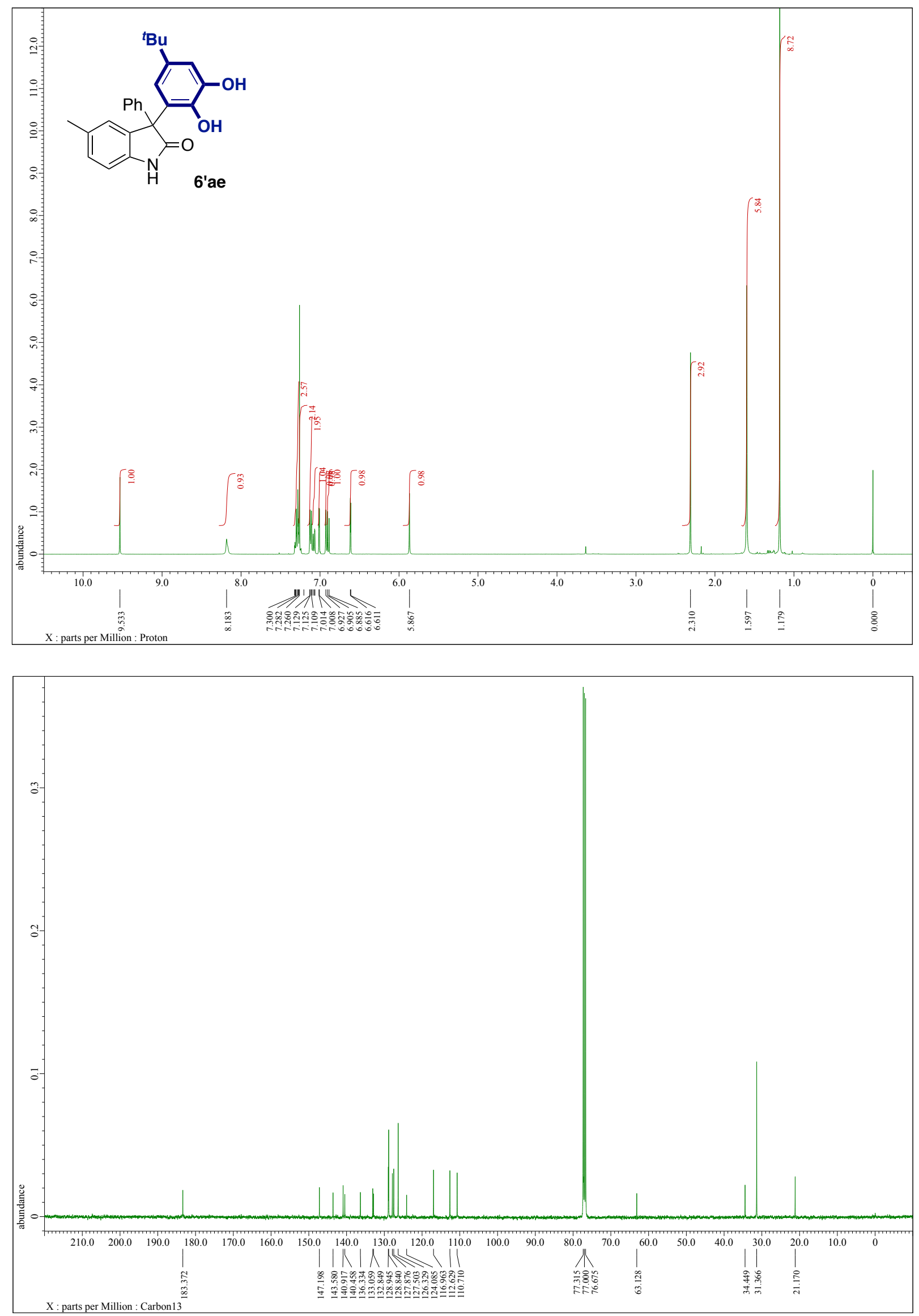

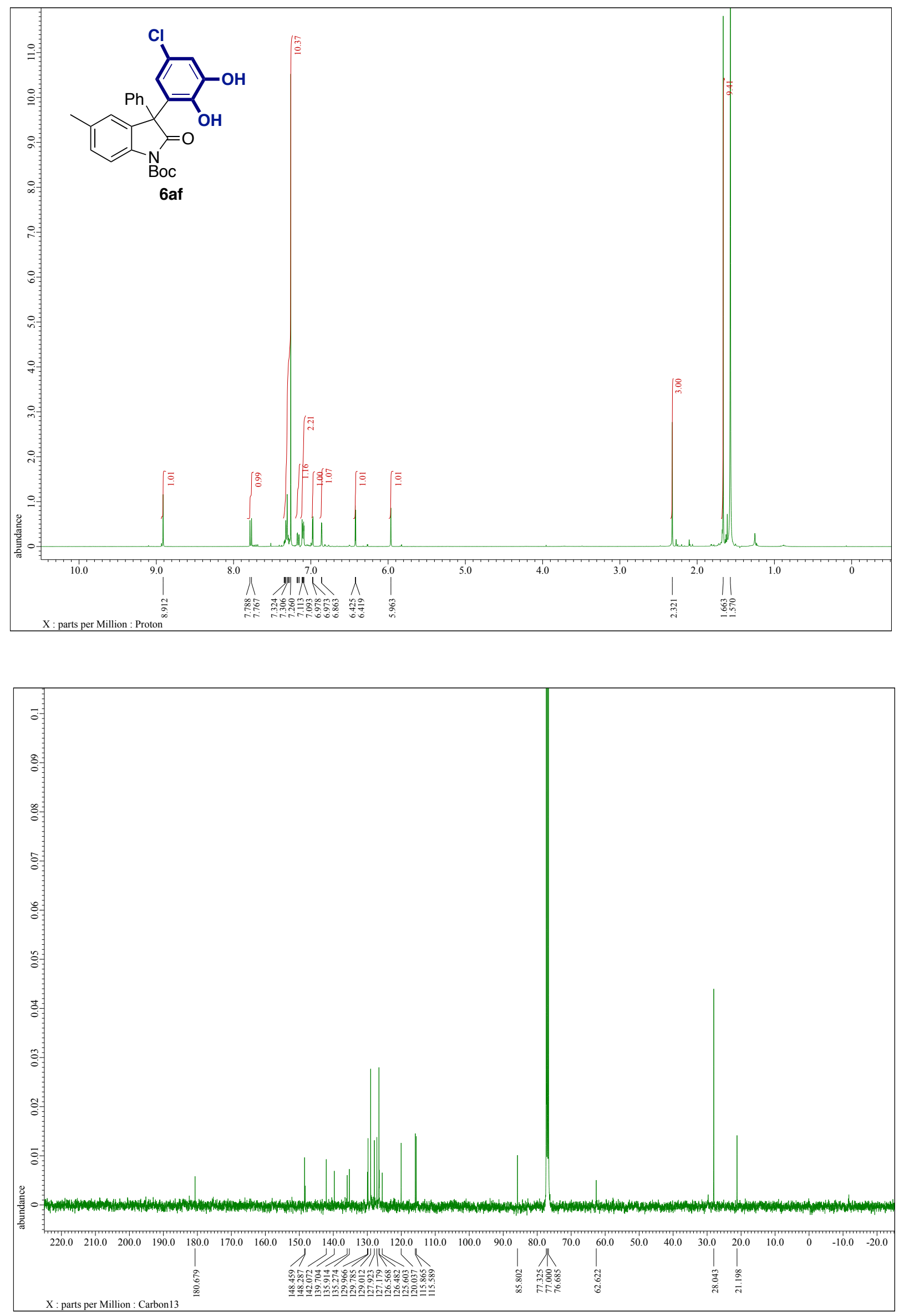

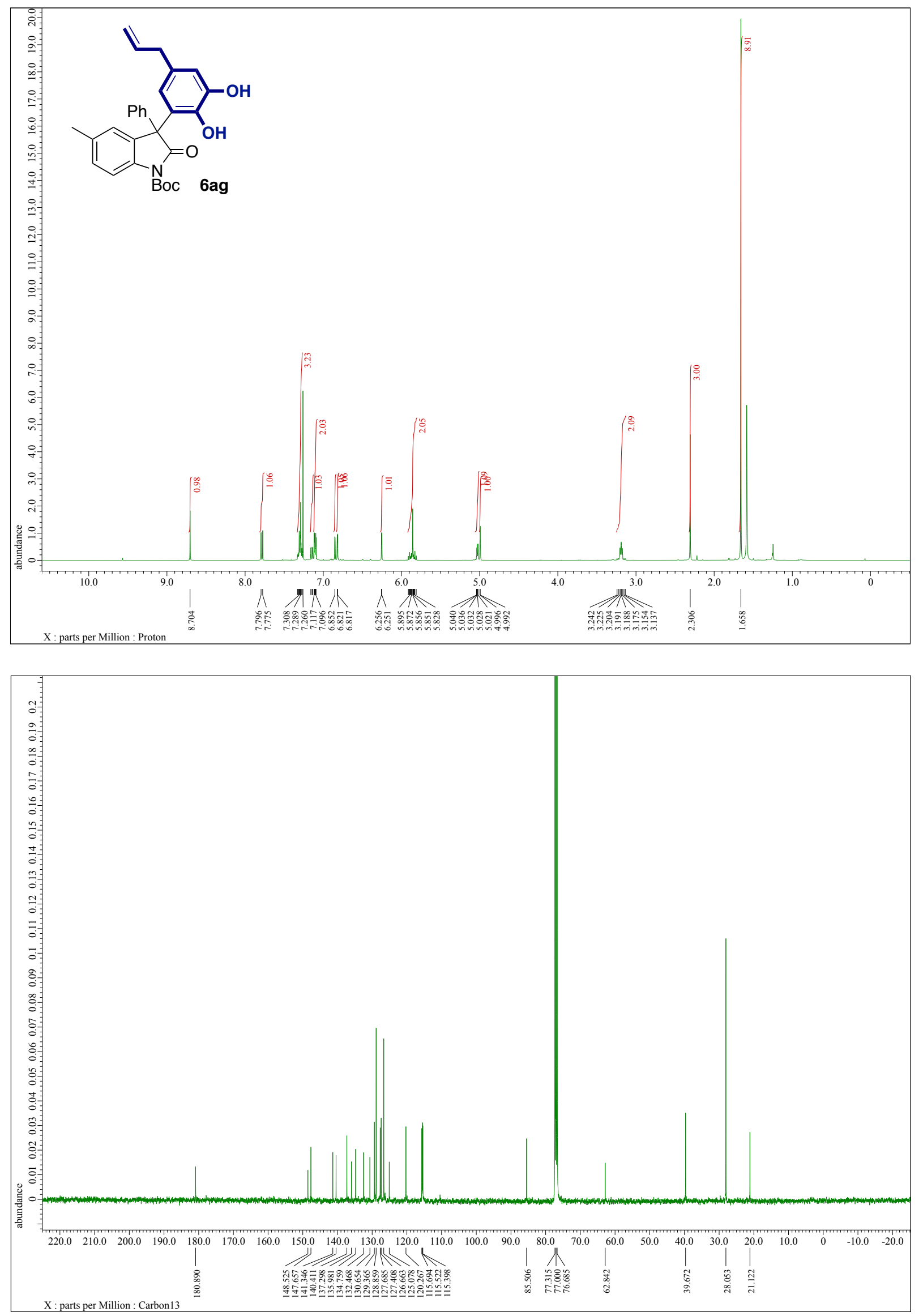

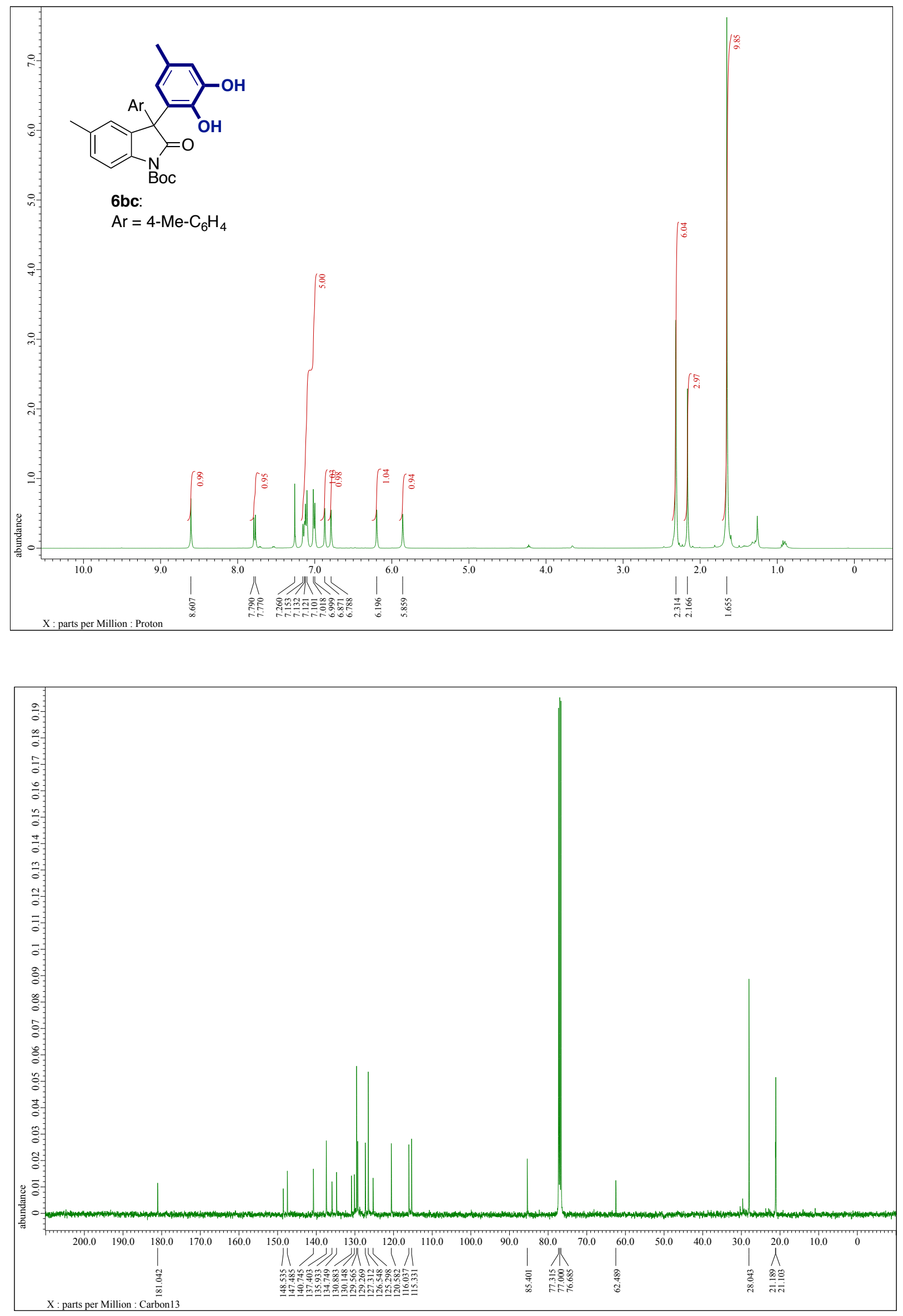

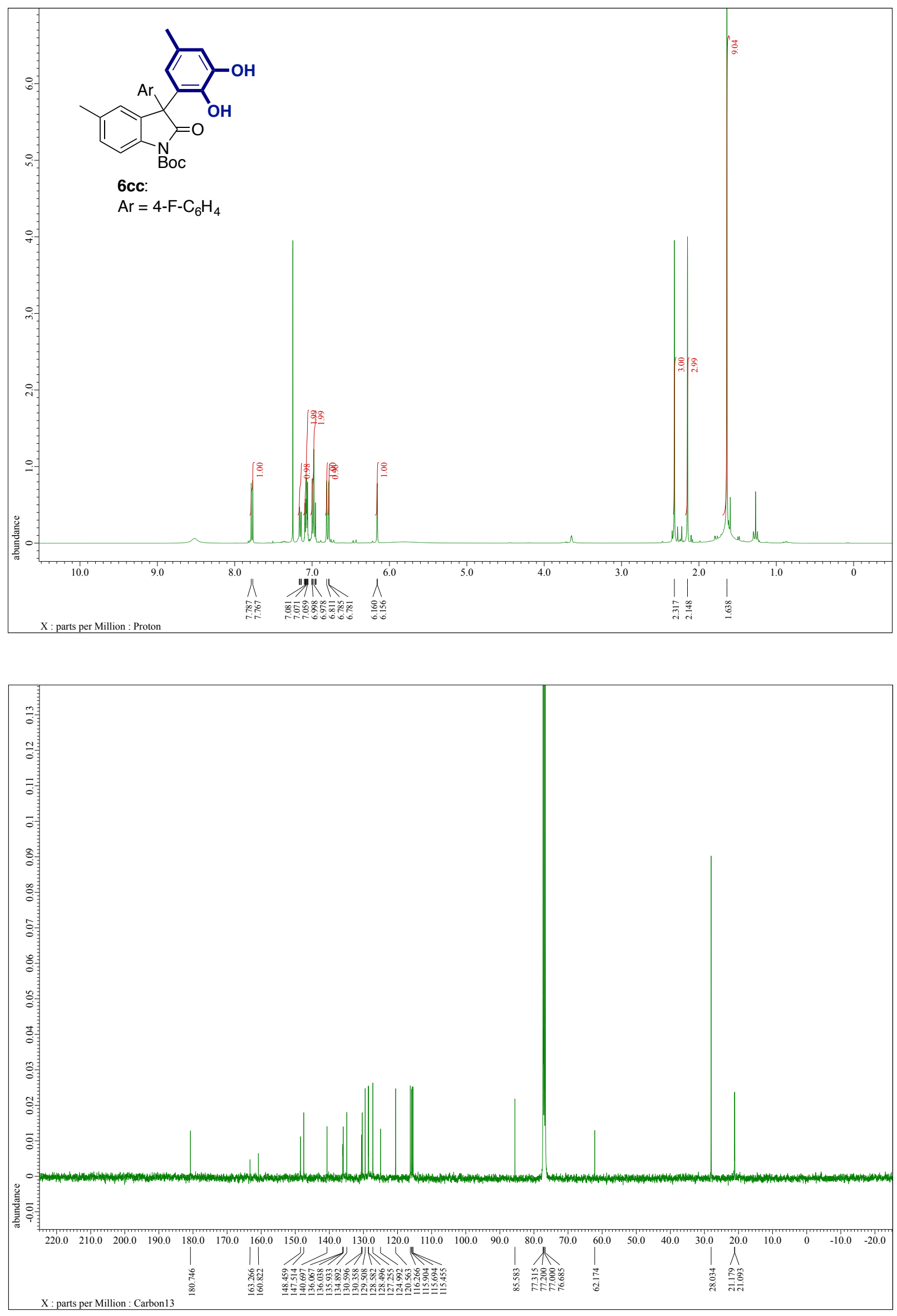

S28 

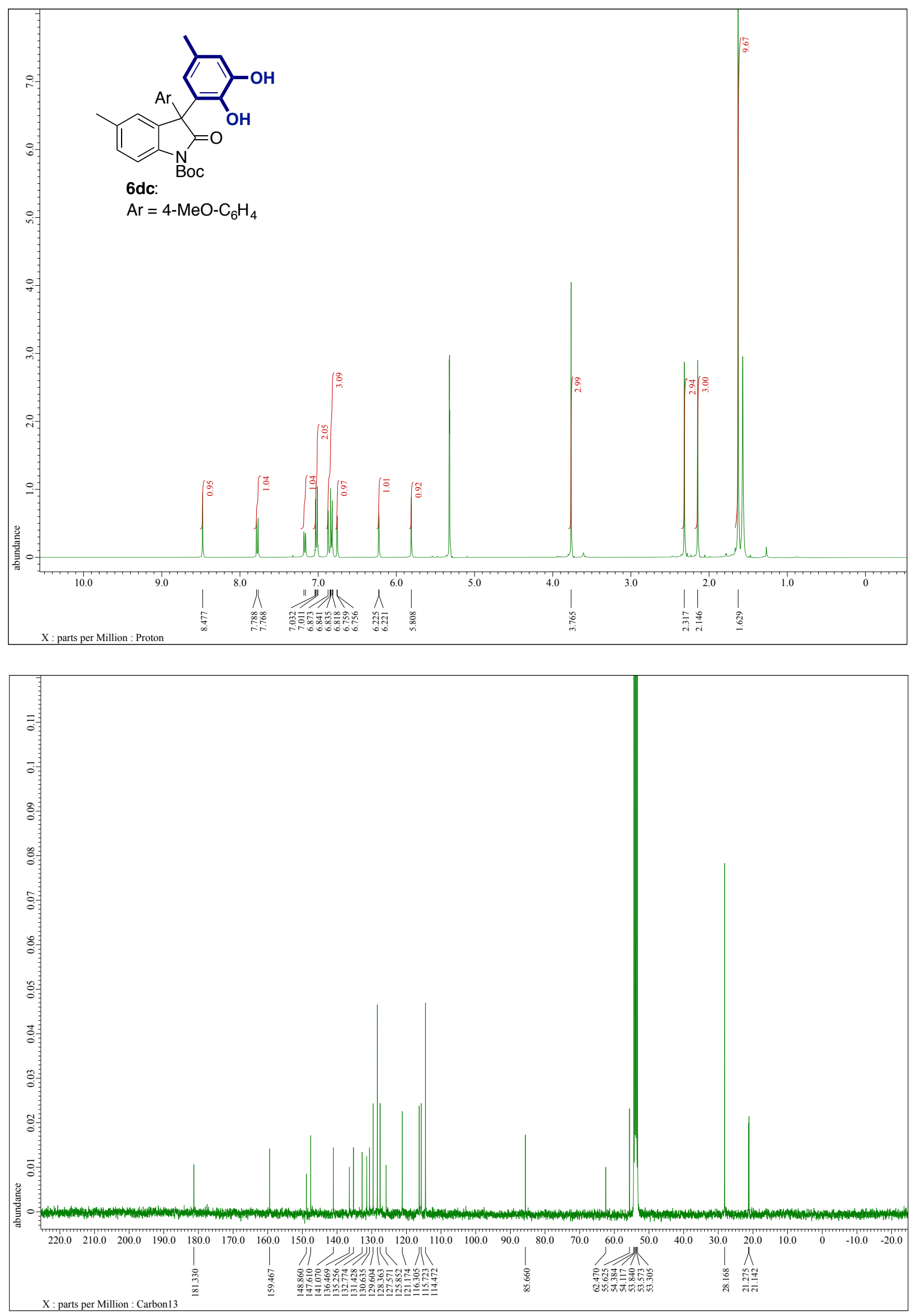

S29 

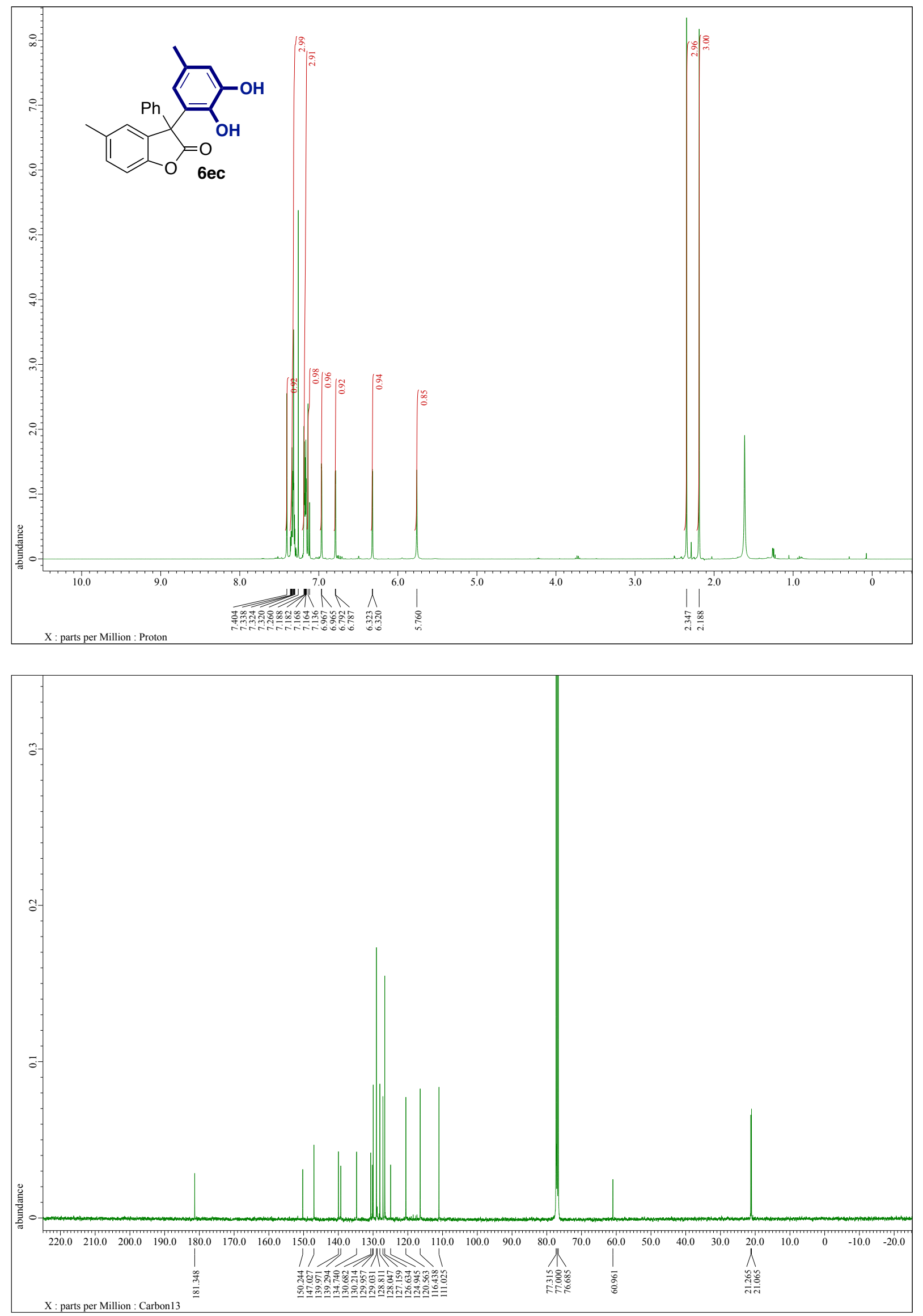

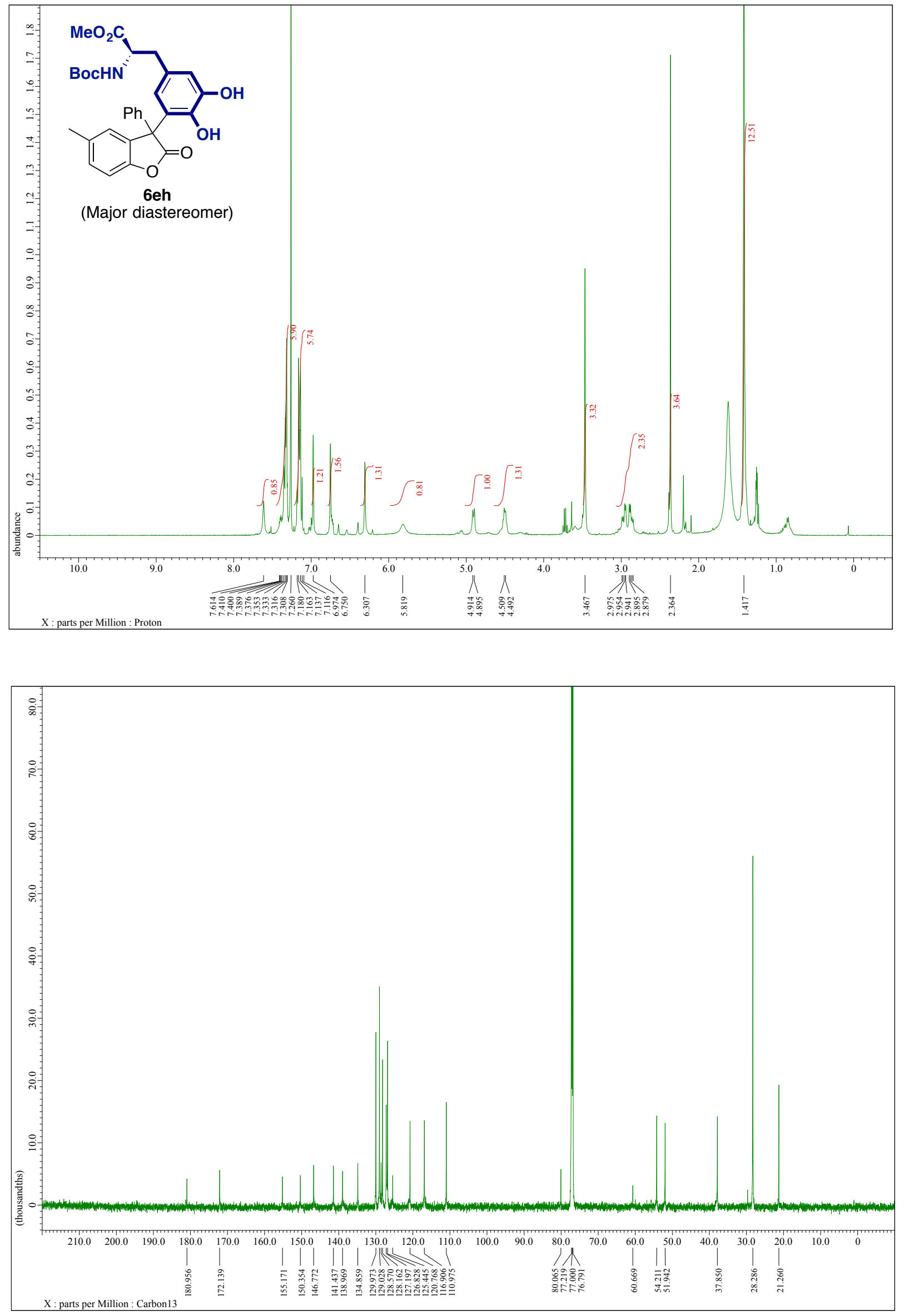

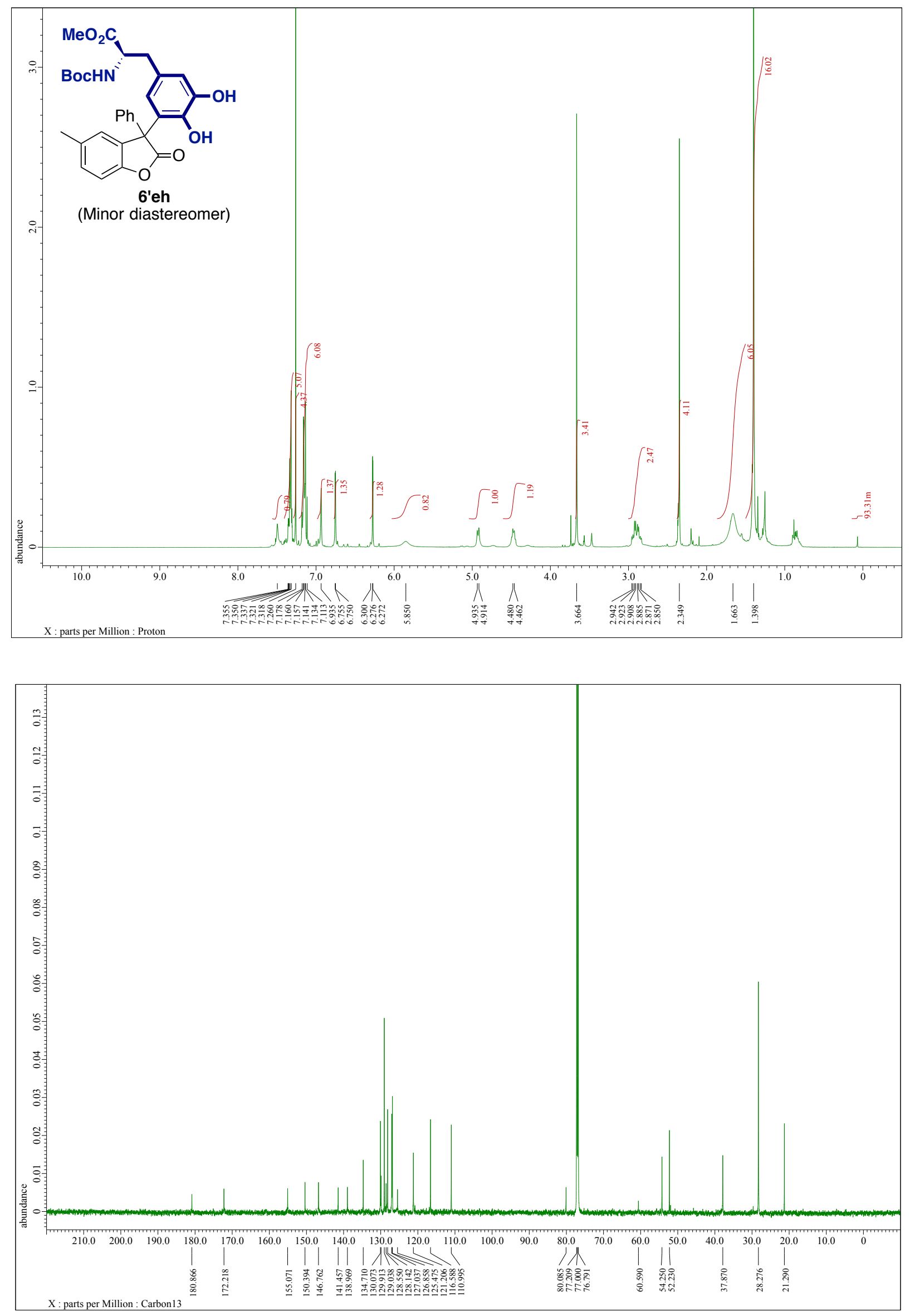

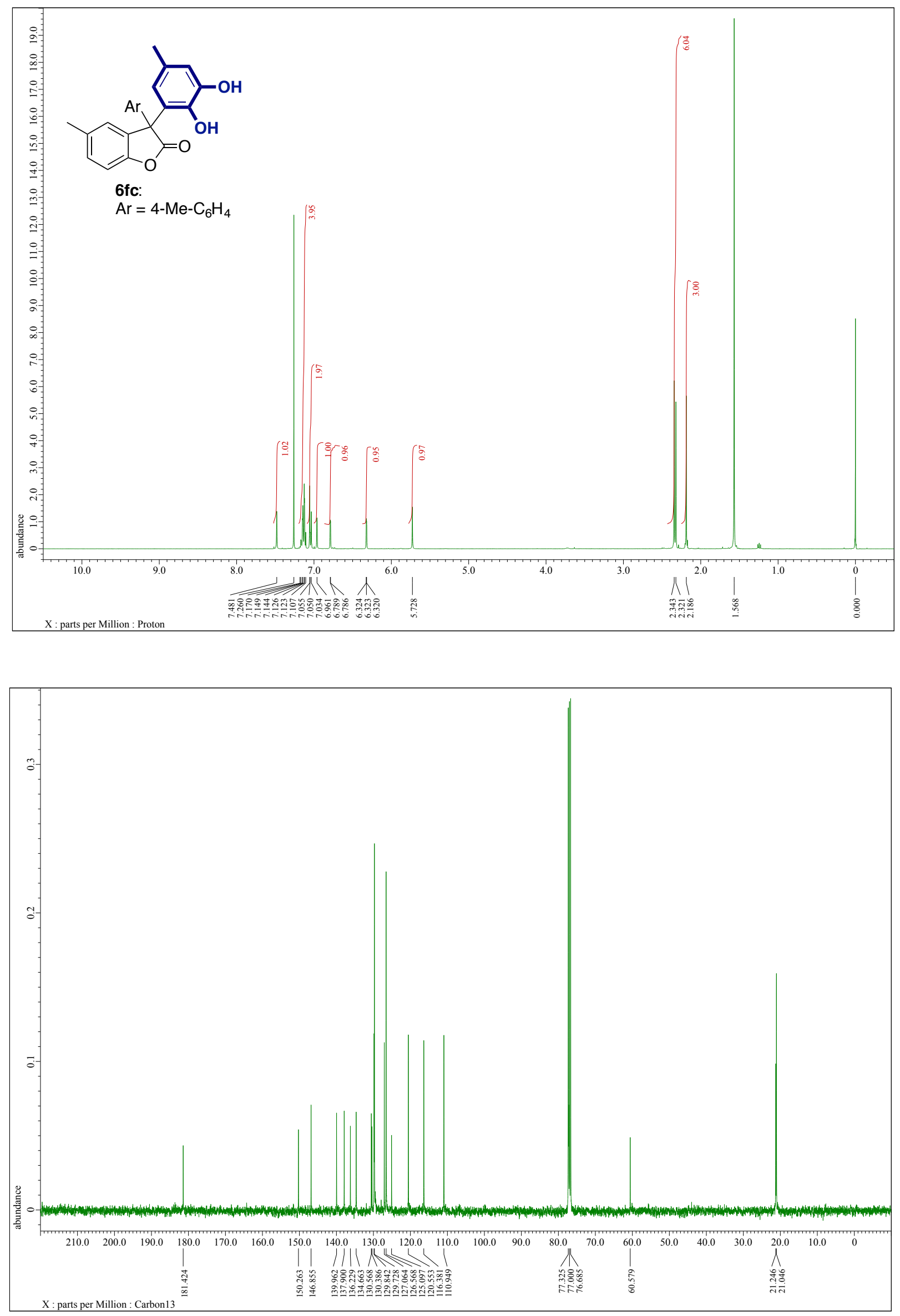

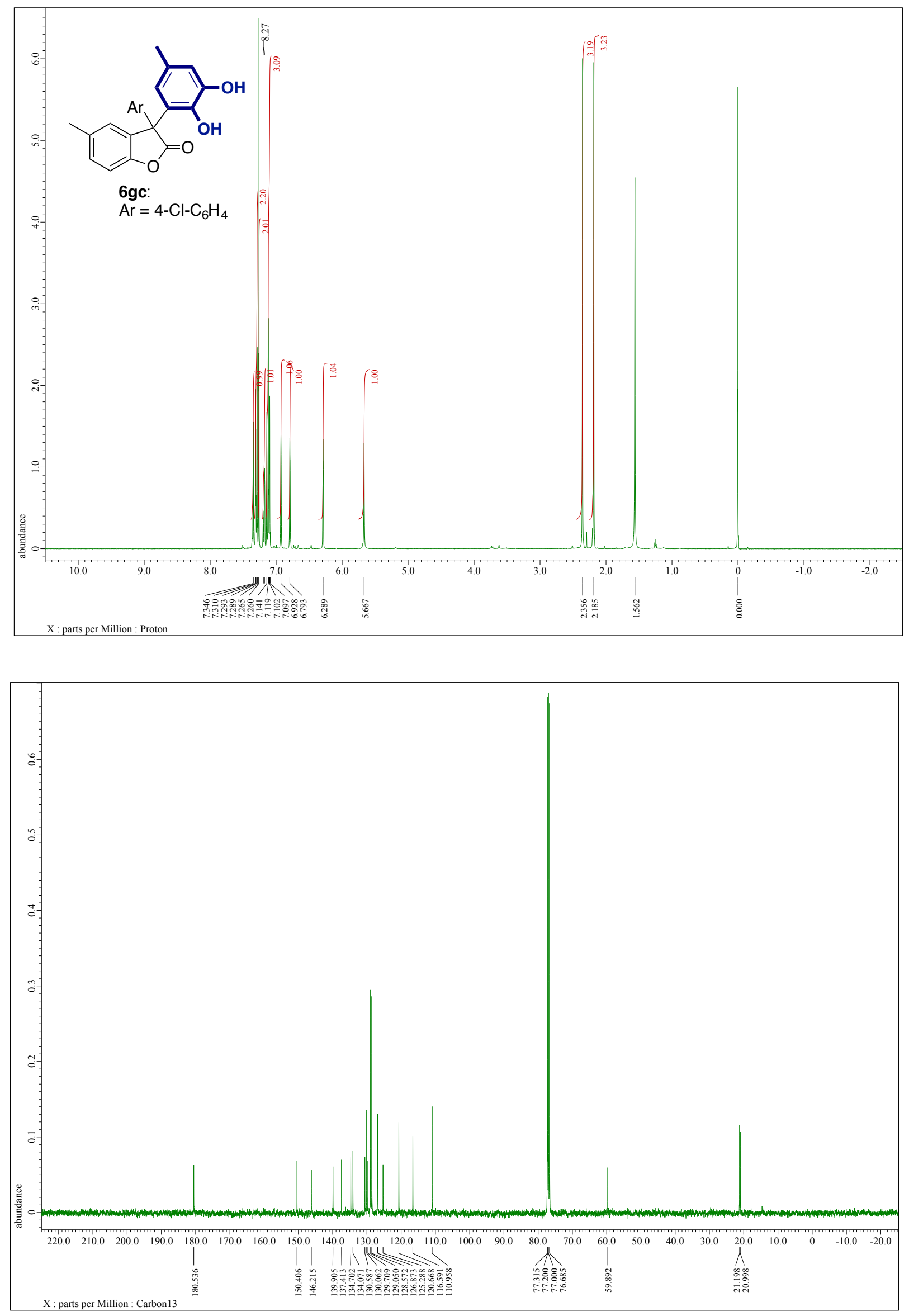

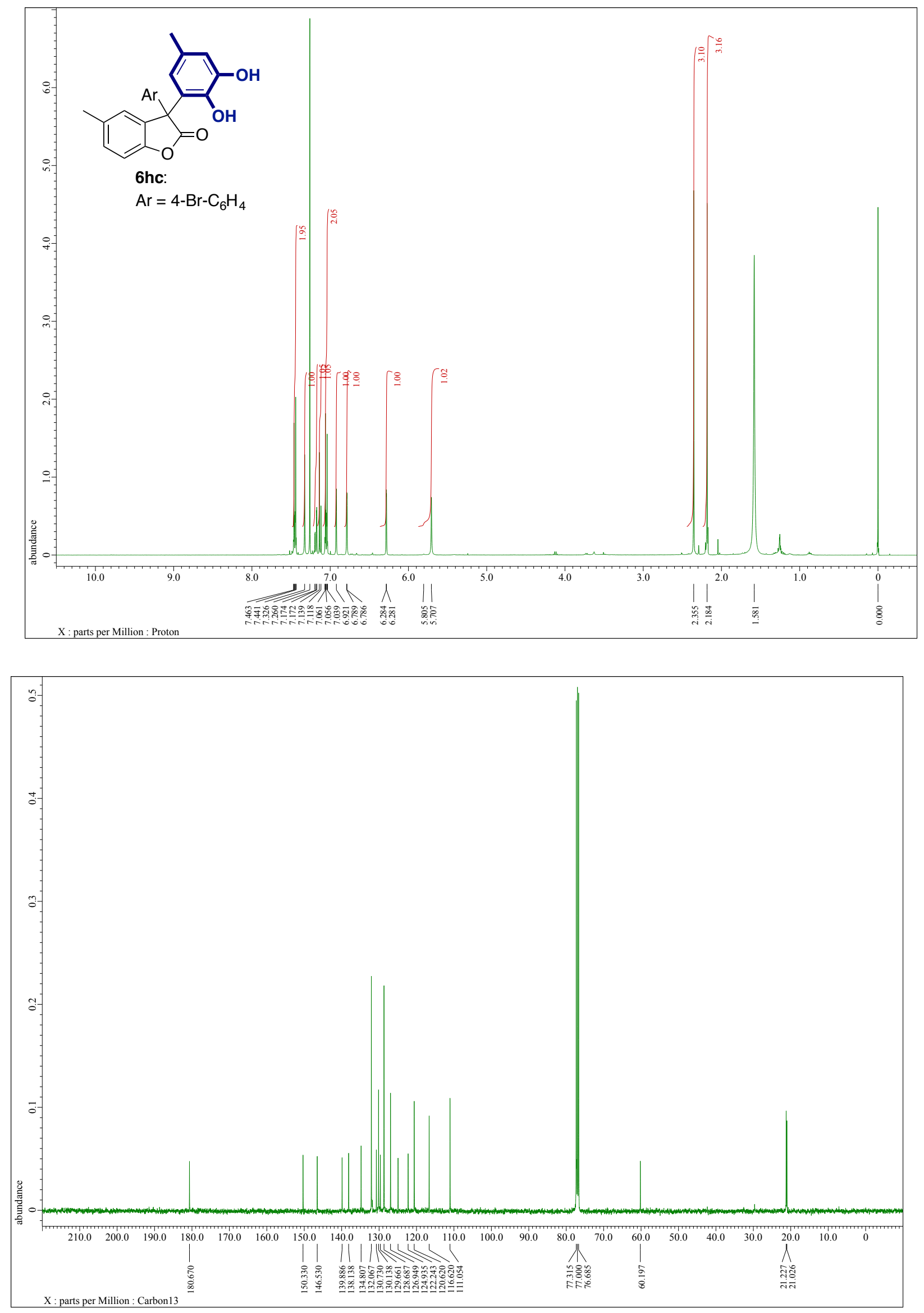

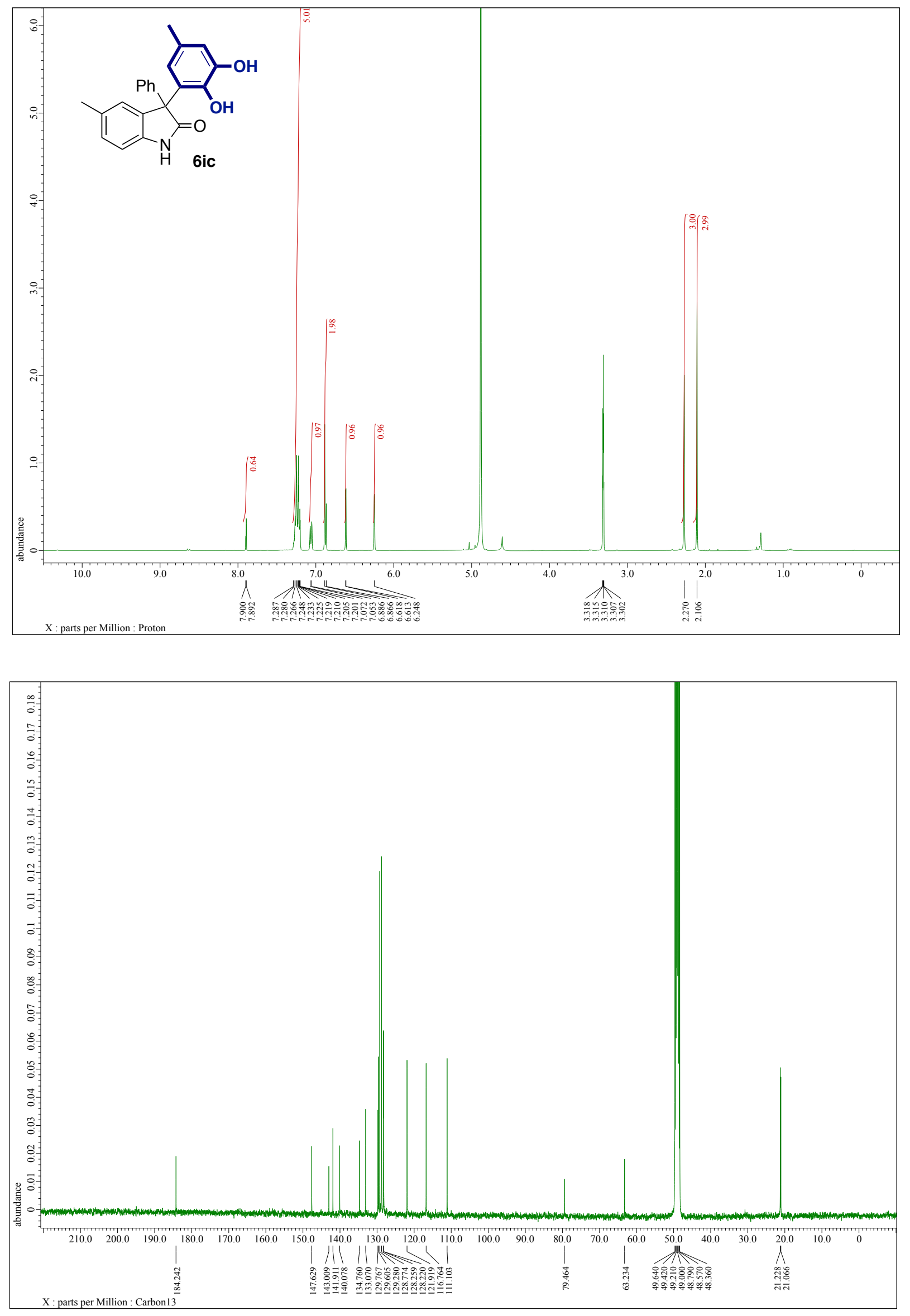

S36 

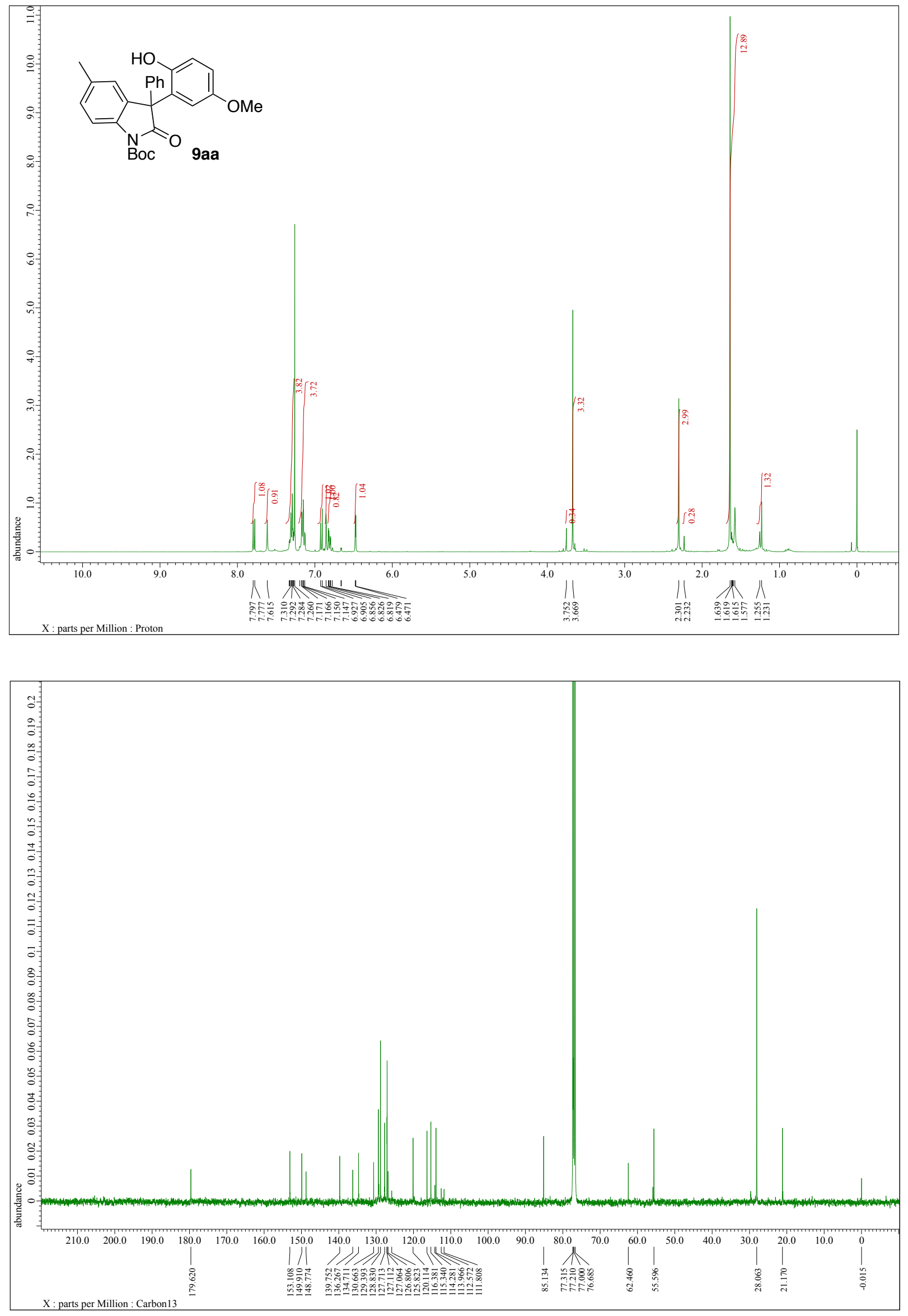

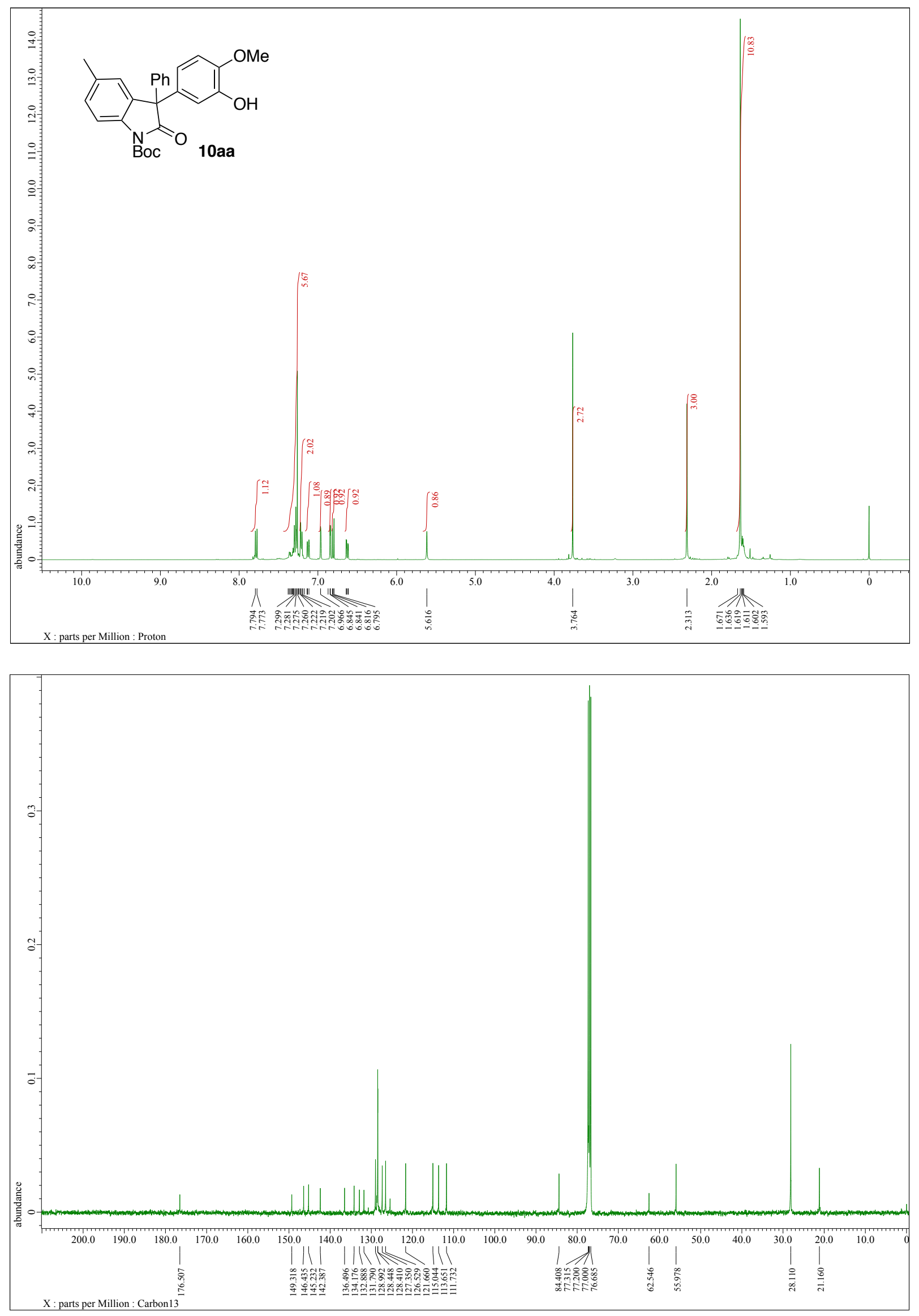

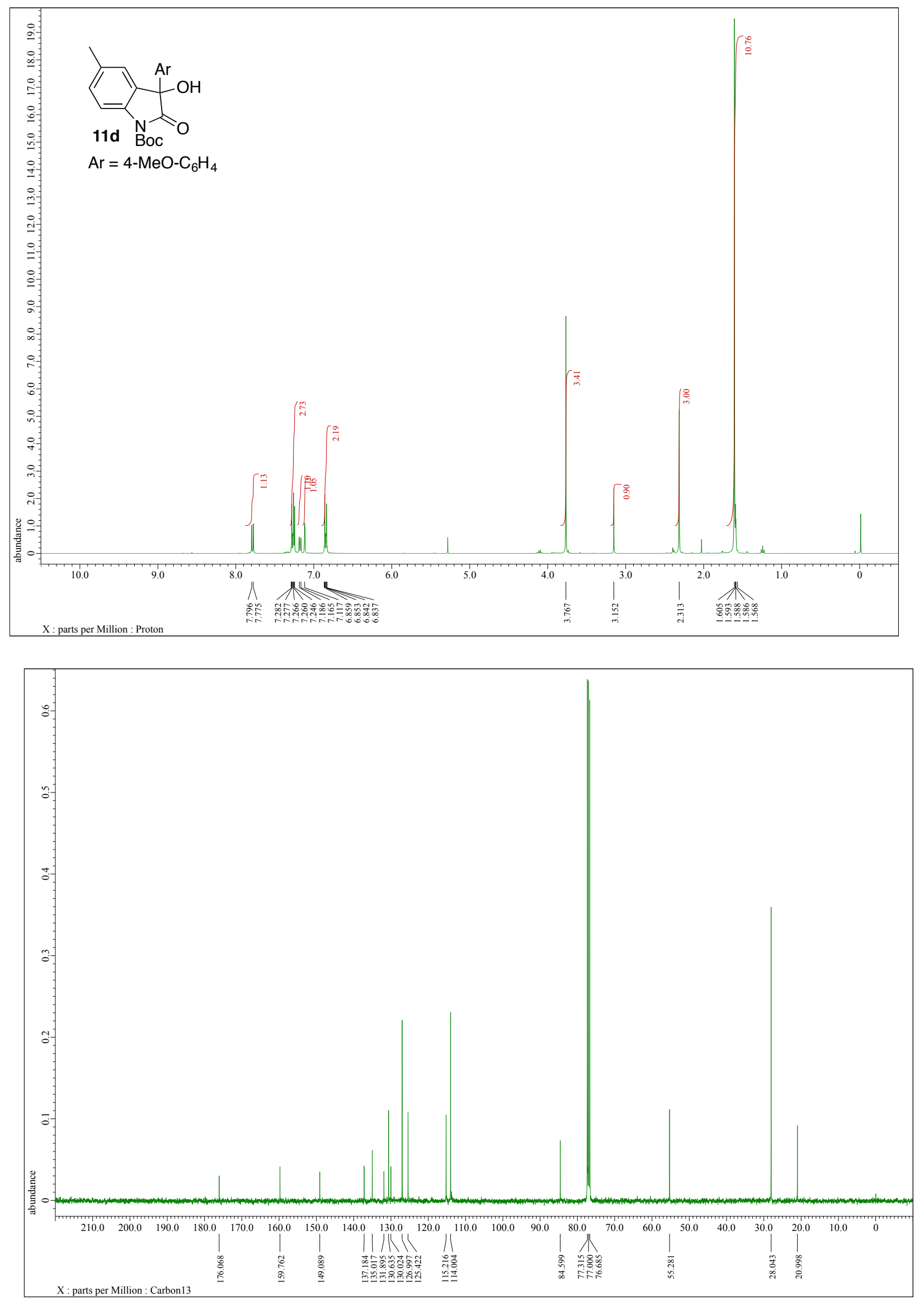

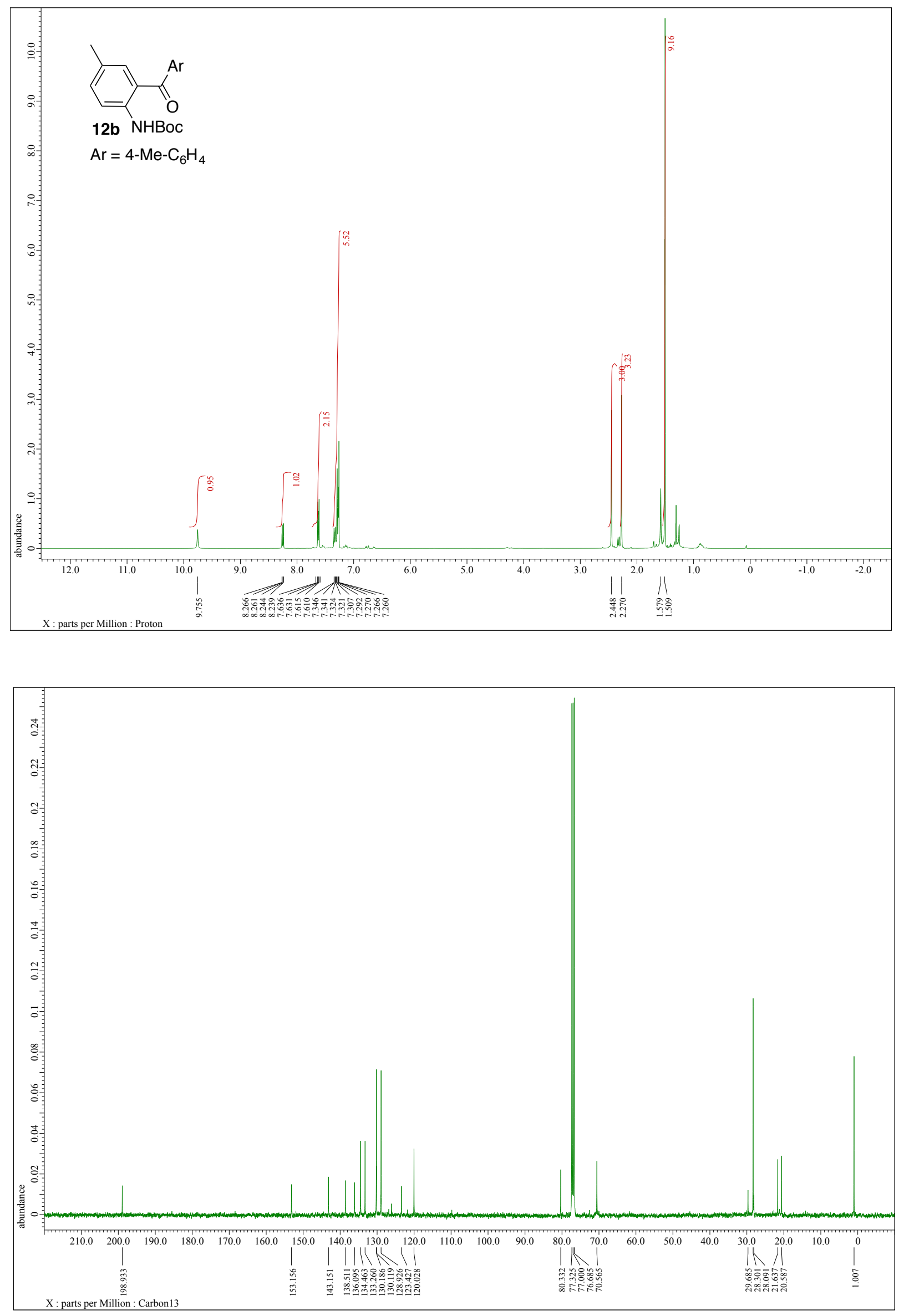

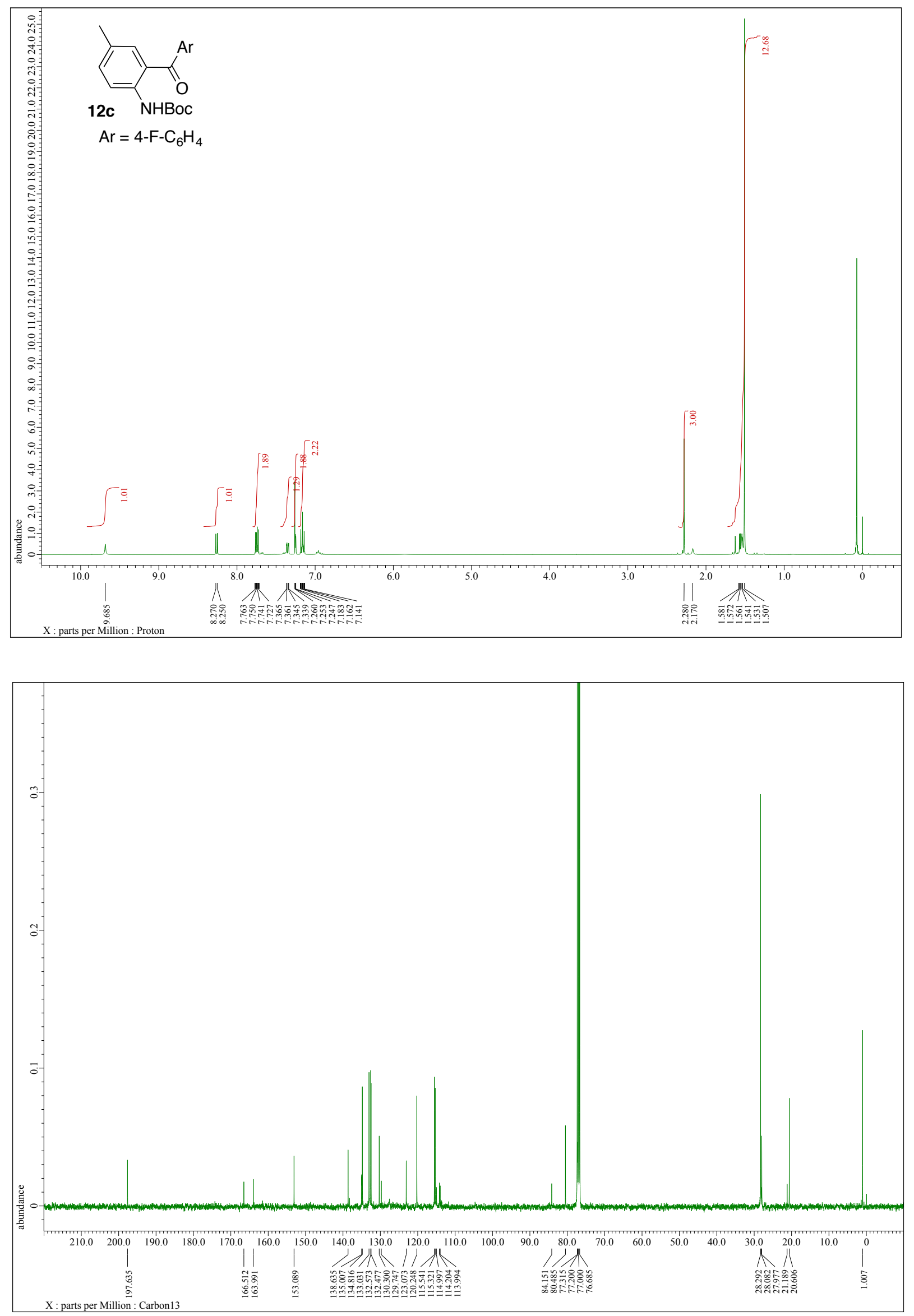

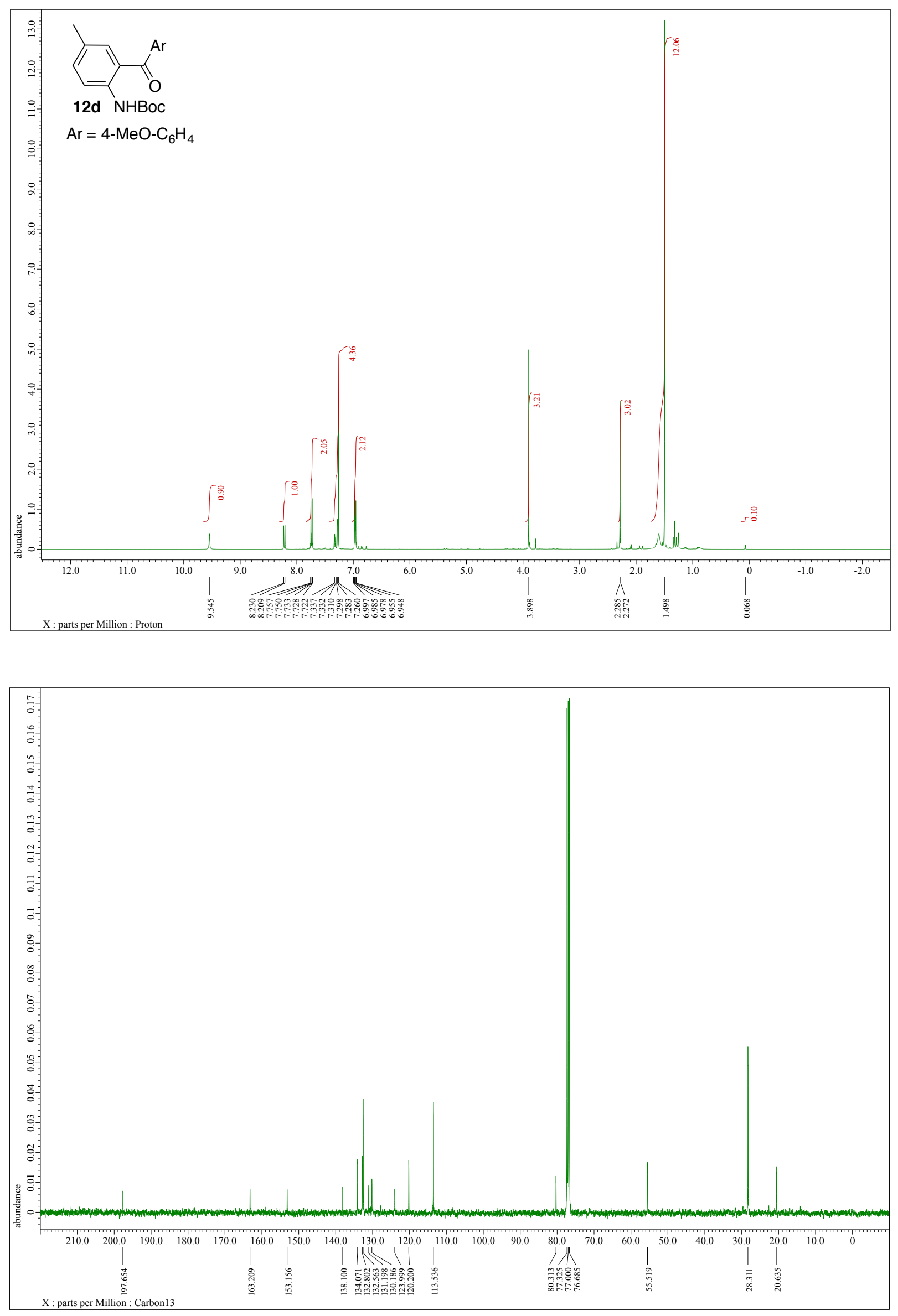

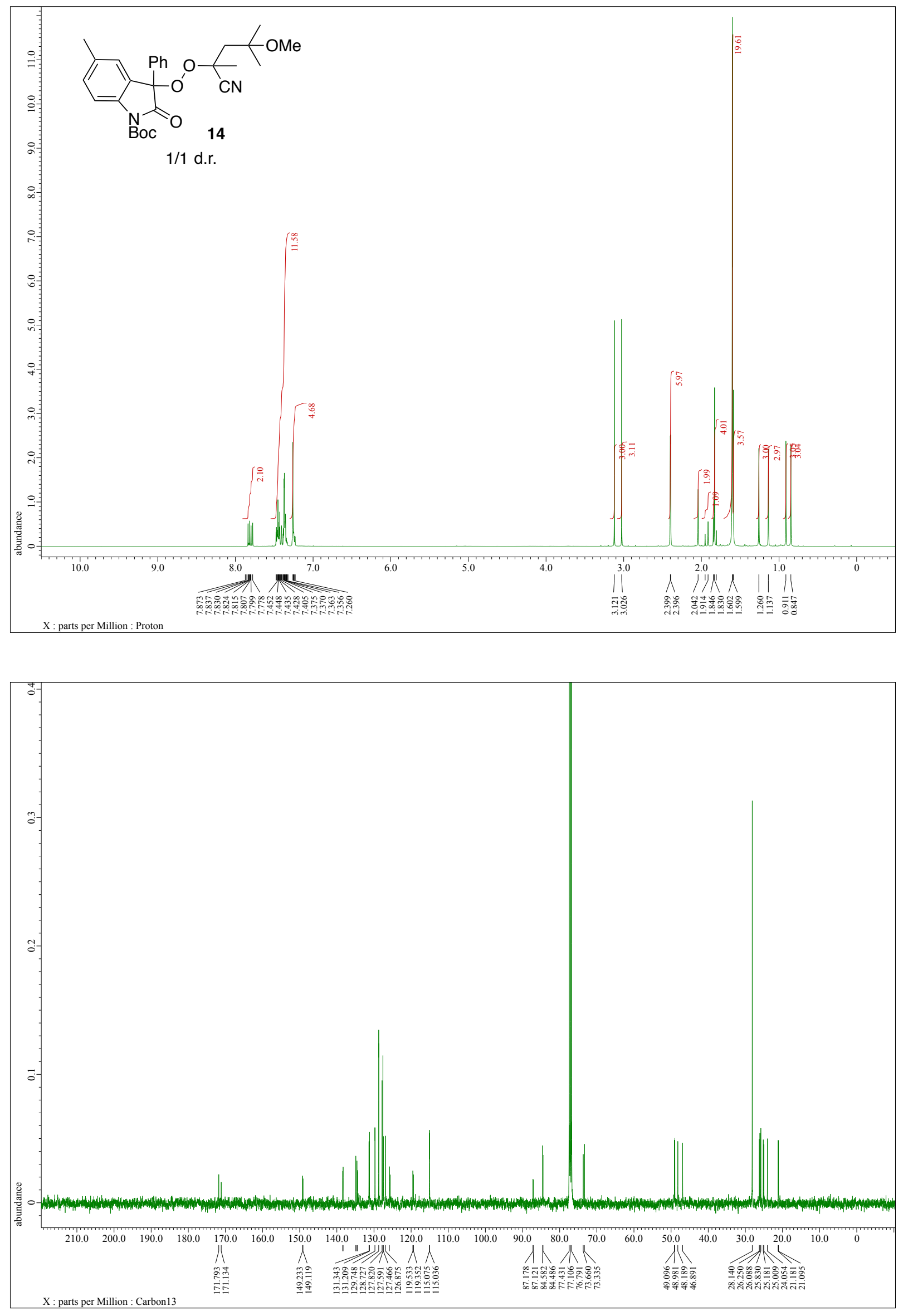


\section{Cartesian coordinates for DFT calculations}

Cartesian coordinates (in $\AA$ ) and energy of $1 \mathrm{e}^{\bullet}$ $\mathrm{E}(\mathrm{UM} 06)=-727.601138719 \mathrm{au}$

\begin{tabular}{|c|c|c|c|c|c|}
\hline \multirow{2}{*}{$\begin{array}{l}\text { Center } \\
\text { number }\end{array}$} & \multirow{2}{*}{$\begin{array}{l}\text { Atomic } \\
\text { number }\end{array}$} & \multirow{2}{*}{$\begin{array}{l}\text { Atomic } \\
\text { type }\end{array}$} & \multicolumn{3}{|c|}{ Coordinates (Angstroms) } \\
\hline & & & $\mathrm{X}$ & $\mathrm{Y}$ & $\mathrm{Z}$ \\
\hline 1 & 8 & 0 & -1.107662 & 2.941633 & -0.088291 \\
\hline 2 & 8 & 0 & 1.073895 & 2.386217 & -0.133485 \\
\hline 3 & 6 & 0 & -0.279434 & 2.075102 & -0.074607 \\
\hline 4 & 6 & 0 & 1.773726 & 1.230408 & -0.084378 \\
\hline 5 & 6 & 0 & 3.152157 & 1.135848 & -0.086694 \\
\hline 6 & 6 & 0 & 3.703736 & -0.142177 & 0.005451 \\
\hline 7 & 1 & 0 & 4.795874 & -0.254249 & 0.008320 \\
\hline 8 & 6 & 0 & 2.905517 & -1.301365 & 0.114264 \\
\hline 9 & 6 & 0 & 1.520403 & -1.174746 & 0.113160 \\
\hline 10 & 1 & 0 & 0.901754 & -2.071360 & 0.231992 \\
\hline 11 & 6 & 0 & 0.926783 & 0.099787 & -0.006286 \\
\hline 12 & 6 & 0 & 3.563560 & -2.644171 & 0.232153 \\
\hline 13 & 6 & 0 & -1.765331 & -1.431553 & -0.428222 \\
\hline 14 & 1 & 0 & -0.876802 & -1.944489 & -0.810427 \\
\hline 15 & 6 & 0 & -2.982463 & -2.098547 & -0.416163 \\
\hline 16 & 6 & 0 & -4.138446 & -1.443394 & 0.012456 \\
\hline 17 & 6 & 0 & -4.065205 & -0.108634 & 0.413962 \\
\hline 18 & 6 & 0 & -2.852894 & 0.567957 & 0.397452 \\
\hline 19 & 1 & 0 & -2.803010 & 1.615768 & 0.706317 \\
\hline 20 & 6 & 0 & -1.668968 & -0.085453 & -0.012152 \\
\hline 21 & 6 & 0 & -0.402521 & 0.605848 & -0.015831 \\
\hline 22 & 1 & 0 & 3.772111 & 2.035279 & -0.150245 \\
\hline 23 & 1 & 0 & 2.823753 & -3.447860 & 0.392632 \\
\hline 24 & 1 & 0 & 4.136085 & -2.895863 & -0.681445 \\
\hline 25 & 1 & 0 & 4.279366 & -2.672565 & 1.075457 \\
\hline 26 & 1 & 0 & -5.098745 & -1.971593 & 0.024429 \\
\hline 27 & 1 & 0 & -3.034236 & -3.139483 & -0.755477 \\
\hline 28 & 1 & 0 & -4.969739 & 0.414169 & 0.745252 \\
\hline
\end{tabular}

Cartesian coordinates (in $\AA$ ) and energy of $2 \mathrm{e}$ $\mathrm{E}(\mathrm{RM} 06)=-1455.25453686 \mathrm{au}$

\begin{tabular}{|c|c|c|c|c|c|}
\hline \multirow{2}{*}{$\begin{array}{l}\text { Center } \\
\text { number }\end{array}$} & \multirow{2}{*}{$\begin{array}{l}\text { Atomic } \\
\text { number }\end{array}$} & \multirow{2}{*}{$\begin{array}{l}\text { Atomic } \\
\text { type }\end{array}$} & \multicolumn{3}{|c|}{ Coordinates (Angstroms) } \\
\hline & & & $\mathrm{X}$ & $\mathrm{Y}$ & $\mathrm{Z}$ \\
\hline 1 & 8 & 0 & -1.549258 & -2.857071 & 0.650504 \\
\hline 2 & 8 & 0 & 0.653158 & -3.198275 & 0.547080 \\
\hline 3 & 8 & 0 & 0.090167 & -2.033190 & -2.284497 \\
\hline 4 & 8 & 0 & 0.836886 & 0.030653 & -2.722352 \\
\hline 5 & 6 & 0 & -0.452630 & -2.402935 & 0.560589 \\
\hline 6 & 6 & 0 & 1.771940 & -2.418749 & 0.458783 \\
\hline 7 & 6 & 0 & 3.057630 & -2.915919 & 0.353999 \\
\hline 8 & 6 & 0 & 4.092308 & -1.987026 & 0.240126 \\
\hline 9 & 1 & 0 & 5.124895 & -2.346051 & 0.144268 \\
\hline 10 & 6 & 0 & 3.850650 & -0.607881 & 0.233403 \\
\hline 11 & 6 & 0 & 2.529528 & -0.149973 & 0.344558 \\
\hline 12 & 1 & 0 & 2.341604 & 0.930914 & 0.339407 \\
\hline 13 & 6 & 0 & 1.480664 & -1.058340 & 0.459937 \\
\hline 14 & 6 & 0 & 4.978837 & 0.375429 & 0.125407 \\
\hline 15 & 6 & 0 & 0.289518 & 0.457572 & 2.654331 \\
\hline 16 & 1 & 0 & 1.369114 & 0.468973 & 2.473966 \\
\hline 17 & 6 & 0 & -0.217515 & 1.137472 & 3.760349 \\
\hline 18 & 6 & 0 & -1.586187 & 1.134265 & 4.017384 \\
\hline 19 & 6 & 0 & -2.437373 & 0.421477 & 3.174925 \\
\hline 20 & 6 & 0 & -1.931377 & -0.262523 & 2.072315 \\
\hline 21 & 1 & 0 & -2.612524 & -0.842506 & 1.442834 \\
\hline
\end{tabular}




$\begin{array}{rlrrrc}22 & 6 & 0 & -0.559472 & -0.229968 & 1.779274 \\ 23 & 6 & 0 & -0.024714 & -0.911829 & 0.521157 \\ 24 & 6 & 0 & 0.129658 & -0.877071 & -1.995667 \\ 25 & 6 & 0 & 0.825274 & 1.232406 & -2.062274 \\ 26 & 6 & 0 & 1.561538 & 2.336460 & -2.448956 \\ 27 & 6 & 0 & 1.503130 & 3.459833 & -1.621580 \\ 28 & 1 & 0 & 2.079631 & 4.353685 & -1.892079 \\ 29 & 6 & 0 & 0.734505 & 3.478185 & -0.450202 \\ 30 & 6 & 0 & 0.001587 & 2.333346 & -0.100013 \\ 31 & 1 & 0 & -0.584507 & 2.337075 & 0.828022 \\ 32 & 6 & 0 & 0.046488 & 1.203794 & -0.909653 \\ 33 & 6 & 0 & 0.671692 & 4.703721 & 0.413067 \\ 34 & 6 & 0 & -2.711752 & -1.345573 & -1.505864 \\ 35 & 1 & 0 & -2.132769 & -2.236190 & -1.761604 \\ 36 & 6 & 0 & -4.094565 & -1.370640 & -1.665304 \\ 37 & 1 & 0 & -4.573561 & -2.282980 & -2.038043 \\ 38 & 6 & 0 & -4.863064 & -0.251329 & -1.355517 \\ 39 & 6 & 0 & -4.231701 & 0.902828 & -0.899681 \\ 40 & 6 & 0 & -2.848533 & 0.928428 & -0.742010 \\ 41 & 1 & 0 & -2.381223 & 1.850898 & -0.386440 \\ 42 & 6 & 0 & -2.065690 & -0.199075 & -1.022226 \\ 43 & 6 & 0 & -0.547492 & -0.179052 & -0.798707 \\ 44 & 1 & 0 & 3.237604 & -3.995026 & 0.345909 \\ 45 & 1 & 0 & 4.679209 & 1.269895 & -0.451715 \\ 46 & 1 & 0 & 5.310353 & 0.729096 & 1.121800 \\ 47 & 1 & 0 & 5.860180 & -0.069380 & -0.370396 \\ 48 & 1 & 0 & -0.201330 & 5.336086 & 0.157504 \\ 49 & 1 & 0 & 0.575257 & 4.439893 & 1.482355 \\ 50 & 1 & 0 & 1.572447 & 5.332493 & 0.295752 \\ 51 & 1 & 0 & 2.172454 & 2.310656 & -3.356419 \\ 52 & 1 & 0 & -1.988006 & 1.671020 & 4.884601 \\ 53 & 1 & 0 & 0.469309 & 1.669674 & 4.428800 \\ 54 & 1 & 0 & -3.513566 & 0.386739 & 3.379965 \\ 55 & 1 & 0 & -4.817604 & 1.799238 & -0.665987 \\ 56 & 1 & 0 & -5.951881 & -0.275116 & -1.480083 \\ -----------------------------------------------------------------------\end{array}$

Cartesian coordinates (in $\AA$ ) and energy of $\mathbf{4 c}$ $\mathrm{E}(\mathrm{RM} 06)=-421.392049676 \mathrm{au}$

\begin{tabular}{|c|c|c|c|c|c|}
\hline \multirow{2}{*}{$\begin{array}{l}\text { Center } \\
\text { number }\end{array}$} & \multirow{2}{*}{$\begin{array}{l}\text { Atomic } \\
\text { number }\end{array}$} & \multirow{2}{*}{$\begin{array}{l}\text { Atomic } \\
\text { type }\end{array}$} & \multicolumn{3}{|c|}{ Coordinates (Angstroms) } \\
\hline & & & $\mathrm{X}$ & $\mathrm{Y}$ & $\mathrm{Z}$ \\
\hline 1 & 8 & 0 & -1.613400 & -1.802292 & 0.000044 \\
\hline 2 & 1 & 0 & -2.507796 & -1.415969 & 0.000101 \\
\hline 3 & 6 & 0 & -0.718897 & -0.792082 & 0.000028 \\
\hline 4 & 6 & 0 & 0.643956 & -1.056786 & -0.000058 \\
\hline 5 & 6 & 0 & -1.162169 & 0.541805 & 0.000043 \\
\hline 6 & 6 & 0 & 1.587601 & -0.019613 & -0.000133 \\
\hline 7 & 1 & 0 & 0.960891 & -2.107344 & -0.000109 \\
\hline 8 & 6 & 0 & -0.240737 & 1.577192 & -0.000061 \\
\hline 9 & 6 & 0 & 1.129480 & 1.297908 & -0.000167 \\
\hline 10 & 1 & 0 & -0.598015 & 2.616213 & -0.000096 \\
\hline 11 & 1 & 0 & 1.849523 & 2.125324 & -0.000287 \\
\hline 12 & 8 & 0 & -2.518527 & 0.701431 & 0.000152 \\
\hline 13 & 1 & 0 & -2.747224 & 1.643532 & -0.000291 \\
\hline 14 & 6 & 0 & 3.052689 & -0.341113 & 0.000096 \\
\hline 15 & 1 & 0 & 3.338962 & -0.934293 & 0.889985 \\
\hline 16 & 1 & 0 & 3.338081 & -0.939605 & -0.886487 \\
\hline 17 & 1 & 0 & 3.669464 & 0.575171 & -0.002875 \\
\hline
\end{tabular}

Cartesian coordinates (in $\AA$ ) and energy of $\mathrm{O}_{2}$

$\mathrm{E}(\mathrm{UM} 06)=-150.131519374$ au

\begin{tabular}{cccccc}
$\begin{array}{l}\text { Center } \\
\text { number }\end{array}$ & $\begin{array}{c}\text { Atomic } \\
\text { number }\end{array}$ & $\begin{array}{c}\text { Atomic } \\
\text { type }\end{array}$ & $X$ & \multicolumn{2}{c}{$\begin{array}{c}\text { Coordinates (Angstroms) } \\
\text { Y }\end{array}$} \\
\hline 1 & 8 & 0 & 0.000000 & 0.000000 & 0.596528 \\
2 & 8 & 0 & 0.000000 & 0.000000 & -0.596528
\end{tabular}


Cartesian coordinates (in $\AA$ ) and energy of $\mathrm{H}_{2} \mathrm{O}$

$\mathrm{E}(\mathrm{RM} 06)=-76.3059331791 \mathrm{au}$

\begin{tabular}{cccccc}
$\begin{array}{l}\text { Center } \\
\text { number }\end{array}$ & $\begin{array}{c}\text { Atomic } \\
\text { number }\end{array}$ & $\begin{array}{c}\text { Atomic } \\
\text { type }\end{array}$ & $\mathrm{X}$ & \multicolumn{3}{c}{ Coordinates (Angstroms) } \\
-1 & 8 & 0 & -0.000000 & -0.000000 & 0.118793 \\
2 & 1 & 0 & -0.000000 & -0.766621 & -0.473621 \\
3 & 1 & 0 & 0.000000 & 0.766621 & -0.473621
\end{tabular}

Cartesian coordinates (in $\AA$ ) and energy of 5ec

$\mathrm{E}(\mathrm{RM} 06)=-2296.89546352 \mathrm{au}$

\begin{tabular}{|c|c|c|c|c|c|}
\hline \multirow{2}{*}{$\begin{array}{l}\text { Center } \\
\text { number }\end{array}$} & \multirow{2}{*}{$\begin{array}{l}\text { Atomic } \\
\text { number }\end{array}$} & \multirow{2}{*}{$\begin{array}{l}\text { Atomic } \\
\text { type }\end{array}$} & \multicolumn{3}{|c|}{ Coordinates (Angstroms) } \\
\hline & & & $\mathrm{X}$ & Y & $\mathrm{Z}$ \\
\hline 1 & 8 & 0 & -1.852025 & 0.917514 & 2.025357 \\
\hline 2 & 8 & 0 & 0.021978 & -2.639684 & -2.597079 \\
\hline 3 & 1 & 0 & 0.497416 & -1.790650 & -2.737019 \\
\hline 4 & 8 & 0 & -0.355465 & -4.721537 & -0.956825 \\
\hline 5 & 1 & 0 & 0.194857 & -4.573171 & -1.750561 \\
\hline 6 & 6 & 0 & -2.890551 & 0.722468 & 1.457727 \\
\hline 7 & 6 & 0 & -3.113698 & 0.155541 & 0.041614 \\
\hline 8 & 6 & 0 & -4.610443 & 0.002781 & 0.047356 \\
\hline 9 & 6 & 0 & -5.484614 & -0.599705 & -0.840787 \\
\hline 10 & 1 & 0 & -5.098682 & -1.084892 & -1.748238 \\
\hline 11 & 6 & 0 & -6.861880 & -0.594622 & -0.565768 \\
\hline 12 & 6 & 0 & -7.314352 & 0.015855 & 0.612284 \\
\hline 13 & 1 & 0 & -8.389828 & 0.011388 & 0.830396 \\
\hline 14 & 6 & 0 & -6.441050 & 0.614749 & 1.525540 \\
\hline 15 & 1 & 0 & -6.793905 & 1.067964 & 2.456906 \\
\hline 16 & 6 & 0 & -5.097642 & 0.587708 & 1.207152 \\
\hline 17 & 6 & 0 & -2.375241 & -1.160286 & -0.201989 \\
\hline 18 & 6 & 0 & -1.552645 & -1.304385 & -1.323627 \\
\hline 19 & 1 & 0 & -1.392133 & -0.472161 & -2.015342 \\
\hline 20 & 6 & 0 & -0.870595 & -2.484038 & -1.579876 \\
\hline 21 & 6 & 0 & -1.050142 & -3.584214 & -0.738464 \\
\hline 22 & 6 & 0 & -1.894750 & -3.464393 & 0.358401 \\
\hline 23 & 6 & 0 & -2.553660 & -2.267687 & 0.658492 \\
\hline 24 & 6 & 0 & -2.665452 & 1.320240 & -0.861207 \\
\hline 25 & 6 & 0 & -1.312230 & 1.683014 & -0.885708 \\
\hline 26 & 1 & 0 & -0.579250 & 1.108728 & -0.308661 \\
\hline 27 & 6 & 0 & -0.869915 & 2.746337 & -1.664092 \\
\hline 28 & 1 & 0 & 0.199056 & 2.994532 & -1.669681 \\
\hline 29 & 6 & 0 & -1.779043 & 3.479802 & -2.427029 \\
\hline 30 & 1 & 0 & -1.434333 & 4.320424 & -3.040143 \\
\hline 31 & 6 & 0 & -3.126885 & 3.135432 & -2.403246 \\
\hline 32 & 1 & 0 & -3.851906 & 3.704854 & -2.996365 \\
\hline 33 & 6 & 0 & -3.569253 & 2.064523 & -1.624682 \\
\hline 34 & 1 & 0 & -4.636036 & 1.815956 & -1.618020 \\
\hline 35 & 8 & 0 & 1.380193 & -0.335274 & -1.922017 \\
\hline 36 & 8 & 0 & -0.143402 & 3.071230 & 2.207406 \\
\hline 37 & 1 & 0 & -0.715098 & 2.279347 & 2.317034 \\
\hline 38 & 8 & 0 & 1.071633 & 5.169342 & 1.127552 \\
\hline 39 & 1 & 0 & 0.281819 & 5.056713 & 1.691148 \\
\hline 40 & 6 & 0 & 2.482895 & -0.443091 & -1.461436 \\
\hline 41 & 6 & 0 & 2.961824 & -0.065091 & -0.051512 \\
\hline 42 & 6 & 0 & 4.450044 & -0.299412 & -0.197588 \\
\hline 43 & 6 & 0 & 5.524897 & -0.080339 & 0.648433 \\
\hline 44 & 1 & 0 & 5.376229 & 0.391235 & 1.628892 \\
\hline 45 & 6 & 0 & 6.818776 & -0.432382 & 0.233829 \\
\hline 46 & 6 & 0 & 6.993676 & -1.003795 & -1.033910 \\
\hline 47 & 1 & 0 & 8.006688 & -1.272268 & -1.359161 \\
\hline 48 & 6 & 0 & 5.919805 & -1.235200 & -1.897571 \\
\hline 49 & 1 & 0 & 6.055384 & -1.668461 & -2.893194 \\
\hline 50 & 6 & 0 & 4.667921 & -0.874450 & -1.441552 \\
\hline
\end{tabular}




\begin{tabular}{|c|c|c|c|c|c|}
\hline 51 & 6 & 0 & 2.514996 & 1.360554 & 0.273776 \\
\hline 52 & 6 & 0 & 1.415828 & 1.549178 & 1.127385 \\
\hline 53 & 1 & 0 & 0.900610 & 0.688513 & 1.570484 \\
\hline 54 & 6 & 0 & 0.922207 & 2.812694 & 1.416506 \\
\hline 55 & 6 & 0 & 1.541734 & 3.938863 & 0.853759 \\
\hline 56 & 6 & 0 & 2.611256 & 3.757837 & -0.012136 \\
\hline 57 & 6 & 0 & 3.105963 & 2.486690 & -0.337918 \\
\hline 58 & 6 & 0 & 2.460692 & -1.128408 & 0.936512 \\
\hline 59 & 6 & 0 & 1.822631 & -2.301104 & 0.527653 \\
\hline 60 & 1 & 0 & 1.578224 & -2.477587 & -0.527410 \\
\hline 61 & 6 & 0 & 1.480737 & -3.289673 & 1.454139 \\
\hline 62 & 1 & 0 & 0.982572 & -4.199134 & 1.096897 \\
\hline 63 & 6 & 0 & 1.768331 & -3.115422 & 2.802266 \\
\hline 64 & 1 & 0 & 1.496955 & -3.888776 & 3.529934 \\
\hline 65 & 6 & 0 & 2.406011 & -1.946598 & 3.222593 \\
\hline 66 & 1 & 0 & 2.636235 & -1.794809 & 4.283546 \\
\hline 67 & 6 & 0 & 2.752797 & -0.968681 & 2.299099 \\
\hline 68 & 1 & 0 & 3.244557 & -0.050705 & 2.643610 \\
\hline 69 & 6 & 0 & 7.988857 & -0.219054 & 1.147955 \\
\hline 70 & 1 & 0 & 7.888188 & 0.718083 & 1.725270 \\
\hline 71 & 1 & 0 & 8.940695 & -0.174146 & 0.589405 \\
\hline 72 & 1 & 0 & 8.080506 & -1.041956 & 1.883869 \\
\hline 73 & 8 & 0 & 3.502925 & -0.971389 & -2.178994 \\
\hline 74 & 8 & 0 & -4.080412 & 1.053771 & 2.015182 \\
\hline 75 & 1 & 0 & 3.050587 & 4.653383 & -0.468074 \\
\hline 76 & 6 & 0 & 4.201716 & 2.415303 & -1.368250 \\
\hline 77 & 1 & 0 & 5.179497 & 2.108963 & -0.950737 \\
\hline 78 & 1 & 0 & 3.970457 & 1.709067 & -2.189279 \\
\hline 79 & 1 & 0 & 4.342874 & 3.404439 & -1.838303 \\
\hline 80 & 6 & 0 & -3.413723 & -2.226565 & 1.890008 \\
\hline 81 & 1 & 0 & -3.095582 & -1.444180 & 2.606247 \\
\hline 82 & 1 & 0 & -4.482294 & -2.049129 & 1.658587 \\
\hline 83 & 1 & 0 & -3.355135 & -3.185710 & 2.432929 \\
\hline 84 & 1 & 0 & -2.004458 & -4.334786 & 1.016962 \\
\hline 85 & 6 & 0 & -7.828185 & -1.217250 & -1.529480 \\
\hline 86 & 1 & 0 & -7.418218 & -2.142859 & -1.973054 \\
\hline 87 & 1 & 0 & -8.786592 & -1.468833 & -1.041541 \\
\hline 88 & 1 & 0 & -8.059232 & -0.533499 & -2.369889 \\
\hline
\end{tabular}

Cartesian coordinates (in $\AA$ ) and energy of 6ec $\mathrm{E}(\mathrm{RM} 06)=-1148.44099575 \mathrm{au}$

\begin{tabular}{|c|c|c|c|c|c|}
\hline \multirow{2}{*}{$\begin{array}{l}\text { Center } \\
\text { number }\end{array}$} & \multirow{2}{*}{$\begin{array}{l}\text { Atomic } \\
\text { number }\end{array}$} & \multirow{2}{*}{$\begin{array}{l}\text { Atomic } \\
\text { type }\end{array}$} & \multicolumn{3}{|c|}{ Coordinates (Angstroms) } \\
\hline & & & $X$ & Y & $\mathrm{Z}$ \\
\hline 1 & 8 & 0 & -2.593573 & 1.291040 & 0.866711 \\
\hline 2 & 1 & 0 & -1.938392 & 1.534528 & 1.561625 \\
\hline 3 & 8 & 0 & -0.551845 & 1.194871 & 2.646421 \\
\hline 4 & 6 & 0 & 1.024133 & 3.918778 & -0.574885 \\
\hline 5 & 1 & 0 & 1.585923 & 4.769003 & -0.171498 \\
\hline 6 & 6 & 0 & 0.922201 & 2.745224 & 0.167765 \\
\hline 7 & 1 & 0 & 1.418490 & 2.686777 & 1.146387 \\
\hline 8 & 6 & 0 & 0.414296 & 4.009478 & -1.824573 \\
\hline 9 & 1 & 0 & 0.491502 & 4.932920 & -2.409810 \\
\hline 10 & 6 & 0 & -0.296029 & 2.919802 & -2.323731 \\
\hline 11 & 1 & 0 & -0.780875 & 2.982367 & -3.304795 \\
\hline 12 & 6 & 0 & -0.403325 & 1.748274 & -1.577154 \\
\hline 13 & 1 & 0 & -0.975076 & 0.898468 & -1.970482 \\
\hline 14 & 6 & 0 & 0.201650 & 1.651817 & -0.322274 \\
\hline 15 & 6 & 0 & -1.072202 & -0.504446 & 0.172429 \\
\hline 16 & 6 & 0 & -2.341939 & 0.044797 & 0.389107 \\
\hline 17 & 6 & 0 & -3.492787 & -0.691865 & 0.049301 \\
\hline 18 & 6 & 0 & -2.118368 & -2.549694 & -0.666684 \\
\hline 19 & 6 & 0 & -0.982884 & -1.808606 & -0.338208 \\
\hline 20 & 1 & 0 & 0.003320 & -2.261659 & -0.488358 \\
\hline 21 & 6 & 0 & -3.377436 & -1.972391 & -0.474361 \\
\hline 22 & 1 & 0 & -4.297386 & -2.518354 & -0.717214 \\
\hline 23 & 6 & 0 & 2.084145 & -0.546820 & 1.494903 \\
\hline 24 & 6 & 0 & 1.481851 & -0.394606 & 0.254602 \\
\hline
\end{tabular}




\begin{tabular}{cccccc}
25 & 6 & 0 & 2.136687 & -0.840336 & -0.883183 \\
26 & 1 & 0 & 1.680805 & -0.698530 & -1.872981 \\
27 & 6 & 0 & 3.383821 & -1.471831 & -0.763522 \\
28 & 6 & 0 & 3.948442 & -1.619554 & 0.511343 \\
29 & 1 & 0 & 4.928058 & -2.105458 & 0.602832 \\
30 & 6 & 0 & 3.312830 & -1.154965 & 1.665149 \\
31 & 1 & 0 & 3.762292 & -1.252669 & 2.657907 \\
32 & 6 & 0 & -2.006122 & -3.943611 & -1.209043 \\
33 & 1 & 0 & -2.457551 & -4.684027 & -0.520591 \\
34 & 1 & 0 & -0.952844 & -4.235890 & -1.367059 \\
35 & 1 & 0 & -2.533641 & -4.046843 & -2.176527 \\
36 & 6 & 0 & 0.177179 & 0.335895 & 0.454534 \\
37 & 6 & 0 & 0.235805 & 0.587804 & 1.973757 \\
38 & 8 & 0 & -4.701633 & -0.137541 & 0.245831 \\
39 & 1 & 0 & -4.549418 & 0.742336 & 0.644175 \\
40 & 6 & 0 & 4.087496 & -1.994966 & -1.980743 \\
41 & 1 & 0 & 5.175872 & -2.085103 & -1.815611 \\
42 & 1 & 0 & 3.716486 & -3.000058 & -2.262527 \\
43 & 1 & 0 & 3.929055 & -1.337245 & -2.854618 \\
44 & 8 & 0 & 1.331642 & 0.010426 & 2.510034 \\
\hline--------------------------------------------------------1754
\end{tabular}

Cartesian coordinates (in A) and energy of DFT-Pd(II)-I $\mathrm{E}(\mathrm{UM} 06)=-3776.91766541 \mathrm{au}$

\begin{tabular}{|c|c|c|c|c|c|}
\hline \multirow{2}{*}{$\begin{array}{l}\text { Center } \\
\text { number }\end{array}$} & \multirow{2}{*}{$\begin{array}{l}\text { Atomic } \\
\text { number }\end{array}$} & \multirow{2}{*}{$\begin{array}{l}\text { Atomic } \\
\text { type }\end{array}$} & \multicolumn{3}{|c|}{ Coordinates (Angstroms) } \\
\hline & & & $\mathrm{X}$ & $\mathrm{Y}$ & $\mathrm{Z}$ \\
\hline 1 & 46 & 0 & -1.510607 & 0.023544 & -0.401019 \\
\hline 2 & 15 & 0 & -3.165494 & 1.474176 & 0.204240 \\
\hline 3 & 15 & 0 & -3.076285 & -1.610843 & -0.124052 \\
\hline 4 & 8 & 0 & 0.009094 & 1.440162 & -0.887765 \\
\hline 5 & 6 & 0 & -4.719804 & -0.787762 & 0.063960 \\
\hline 6 & 6 & 0 & -4.562943 & 0.473081 & 0.896996 \\
\hline 7 & 1 & 0 & -4.297689 & 0.235557 & 1.946179 \\
\hline 8 & 1 & 0 & -5.492034 & 1.072155 & 0.931850 \\
\hline 9 & 1 & 0 & -5.047880 & -0.543911 & -0.967201 \\
\hline 10 & 1 & 0 & -5.472535 & -1.486920 & 0.474367 \\
\hline 11 & 6 & 0 & -3.176374 & -2.683350 & -1.576284 \\
\hline 12 & 6 & 0 & -3.301073 & -4.072821 & -1.464249 \\
\hline 13 & 6 & 0 & -3.167956 & -2.075677 & -2.841492 \\
\hline 14 & 6 & 0 & -3.425895 & -4.847057 & -2.616275 \\
\hline 15 & 1 & 0 & -3.306537 & -4.554319 & -0.478519 \\
\hline 16 & 6 & 0 & -3.295363 & -2.856068 & -3.985522 \\
\hline 17 & 1 & 0 & -3.061544 & -0.983808 & -2.931444 \\
\hline 18 & 6 & 0 & -3.424035 & -4.241598 & -3.871710 \\
\hline 19 & 1 & 0 & -3.529702 & -5.934122 & -2.530951 \\
\hline 20 & 1 & 0 & -3.293787 & -2.382996 & -4.973429 \\
\hline 21 & 1 & 0 & -3.524367 & -4.855630 & -4.773400 \\
\hline 22 & 6 & 0 & -2.735119 & -2.632426 & 1.329310 \\
\hline 23 & 6 & 0 & -3.686476 & -2.878806 & 2.326896 \\
\hline 24 & 6 & 0 & -1.458587 & -3.212772 & 1.413933 \\
\hline 25 & 6 & 0 & -3.364353 & -3.707824 & 3.400590 \\
\hline 26 & 1 & 0 & -4.690126 & -2.439298 & 2.276217 \\
\hline 27 & 6 & 0 & -1.153096 & -4.049018 & 2.483019 \\
\hline 28 & 1 & 0 & -0.710003 & -2.993068 & 0.637359 \\
\hline 29 & 6 & 0 & -2.102749 & -4.296250 & 3.475762 \\
\hline 30 & 1 & 0 & -4.110607 & -3.903886 & 4.178048 \\
\hline 31 & 1 & 0 & -0.158347 & -4.507651 & 2.547025 \\
\hline 32 & 1 & 0 & -1.859490 & -4.956705 & 4.315621 \\
\hline 33 & 6 & 0 & -2.678918 & 2.634003 & 1.510325 \\
\hline 34 & 6 & 0 & -2.791702 & 4.019913 & 1.354930 \\
\hline 35 & 6 & 0 & -2.205056 & 2.099715 & 2.719574 \\
\hline 36 & 6 & 0 & -2.469614 & 4.863300 & 2.418404 \\
\hline 37 & 1 & 0 & -3.149631 & 4.445448 & 0.409081 \\
\hline 38 & 6 & 0 & -1.900141 & 2.946101 & 3.779755 \\
\hline 39 & 1 & 0 & -2.087827 & 1.011536 & 2.836960 \\
\hline 40 & 6 & 0 & -2.040909 & 4.328150 & 3.630942 \\
\hline 41 & 1 & 0 & -2.572990 & 5.947479 & 2.300871 \\
\hline 42 & 1 & 0 & -1.557095 & 2.527563 & 4.732879 \\
\hline 43 & 1 & 0 & -1.812685 & 4.993795 & 4.471437 \\
\hline 44 & 6 & 0 & -3.799602 & 2.399301 & -1.216094 \\
\hline
\end{tabular}




\begin{tabular}{|c|c|c|c|c|c|}
\hline 45 & 6 & 0 & -5.071930 & 2.989634 & -1.192631 \\
\hline 46 & 6 & 0 & -2.983079 & 2.537695 & -2.348049 \\
\hline 47 & 6 & 0 & -5.525737 & 3.701661 & -2.298997 \\
\hline 48 & 1 & 0 & -5.715210 & 2.910376 & -0.306501 \\
\hline 49 & 6 & 0 & -3.445238 & 3.255487 & -3.449565 \\
\hline 50 & 1 & 0 & -1.978085 & 2.092407 & -2.357674 \\
\hline 51 & 6 & 0 & -4.714247 & 3.831355 & -3.427115 \\
\hline 52 & 1 & 0 & -6.520158 & 4.160477 & -2.280607 \\
\hline 53 & 1 & 0 & -2.808646 & 3.364609 & -4.334252 \\
\hline 54 & 1 & 0 & -5.076728 & 4.390571 & -4.296627 \\
\hline 55 & 1 & 0 & 0.015572 & 2.247789 & -0.346403 \\
\hline 56 & 46 & 0 & 1.541359 & 0.072240 & -0.355183 \\
\hline 57 & 15 & 0 & 3.198054 & 1.575051 & 0.071287 \\
\hline 58 & 15 & 0 & 3.028860 & -1.491042 & 0.371970 \\
\hline 59 & 6 & 0 & 4.678976 & -0.675284 & 0.595719 \\
\hline 60 & 6 & 0 & 4.452927 & 0.730799 & 1.132108 \\
\hline 61 & 1 & 0 & 4.026247 & 0.706134 & 2.155585 \\
\hline 62 & 1 & 0 & 5.387459 & 1.319354 & 1.194088 \\
\hline 63 & 1 & 0 & 5.184854 & -0.652634 & -0.389270 \\
\hline 64 & 1 & 0 & 5.309783 & -1.282354 & 1.272283 \\
\hline 65 & 6 & 0 & 3.230661 & -2.908970 & -0.726691 \\
\hline 66 & 6 & 0 & 2.766425 & -4.184021 & -0.382282 \\
\hline 67 & 6 & 0 & 3.789978 & -2.685958 & -1.994560 \\
\hline 68 & 6 & 0 & 2.895390 & -5.234498 & -1.288522 \\
\hline 69 & 1 & 0 & 2.308709 & -4.363015 & 0.598553 \\
\hline 70 & 6 & 0 & 3.918056 & -3.740499 & -2.892011 \\
\hline 71 & 1 & 0 & 4.121657 & -1.680517 & -2.291699 \\
\hline 72 & 6 & 0 & 3.474648 & -5.015638 & -2.537068 \\
\hline 73 & 1 & 0 & 2.542908 & -6.234694 & -1.014506 \\
\hline 74 & 1 & 0 & 4.365106 & -3.567516 & -3.876798 \\
\hline 75 & 1 & 0 & 3.579211 & -5.845883 & -3.244021 \\
\hline 76 & 6 & 0 & 2.543208 & -2.054175 & 2.029073 \\
\hline 77 & 6 & 0 & 3.364257 & -2.944164 & 2.739227 \\
\hline 78 & 6 & 0 & 1.380395 & -1.554175 & 2.629274 \\
\hline 79 & 6 & 0 & 3.024185 & -3.322417 & 4.034585 \\
\hline 80 & 1 & 0 & 4.274906 & -3.351644 & 2.279422 \\
\hline 81 & 6 & 0 & 1.048991 & -1.931652 & 3.929651 \\
\hline 82 & 1 & 0 & 0.723967 & -0.872794 & 2.068064 \\
\hline 83 & 6 & 0 & 1.870033 & -2.811341 & 4.631860 \\
\hline 84 & 1 & 0 & 3.667978 & -4.016438 & 4.585690 \\
\hline 85 & 1 & 0 & 0.136003 & -1.541136 & 4.394087 \\
\hline 86 & 1 & 0 & 1.611445 & -3.104442 & 5.655478 \\
\hline 87 & 6 & 0 & 2.653270 & 3.085687 & 0.903441 \\
\hline 88 & 6 & 0 & 3.024996 & 4.353564 & 0.440755 \\
\hline 89 & 6 & 0 & 1.838377 & 2.961883 & 2.039715 \\
\hline 90 & 6 & 0 & 2.594657 & 5.490611 & 1.122692 \\
\hline 91 & 1 & 0 & 3.654555 & 4.457724 & -0.451635 \\
\hline 92 & 6 & 0 & 1.423881 & 4.101481 & 2.720007 \\
\hline 93 & 1 & 0 & 1.530135 & 1.967194 & 2.395865 \\
\hline 94 & 6 & 0 & 1.800070 & 5.365138 & 2.260323 \\
\hline 95 & 1 & 0 & 2.888623 & 6.482624 & 0.763340 \\
\hline 96 & 1 & 0 & 0.801901 & 4.004652 & 3.616430 \\
\hline 97 & 1 & 0 & 1.470786 & 6.261900 & 2.797137 \\
\hline 98 & 6 & 0 & 3.987966 & 2.033777 & -1.490584 \\
\hline 99 & 6 & 0 & 5.370100 & 2.233299 & -1.602442 \\
\hline 100 & 6 & 0 & 3.157333 & 2.233930 & -2.604145 \\
\hline 101 & 6 & 0 & 5.916891 & 2.625055 & -2.822618 \\
\hline 102 & 1 & 0 & 6.034829 & 2.093468 & -0.740907 \\
\hline 103 & 6 & 0 & 3.712063 & 2.631490 & -3.817439 \\
\hline 104 & 1 & 0 & 2.072031 & 2.081787 & -2.513350 \\
\hline 105 & 6 & 0 & 5.089629 & 2.823893 & -3.927254 \\
\hline 106 & 1 & 0 & 6.997683 & 2.780226 & -2.909292 \\
\hline 107 & 1 & 0 & 3.064630 & 2.790596 & -4.686533 \\
\hline 108 & 1 & 0 & 5.523818 & 3.133241 & -4.884280 \\
\hline 109 & 8 & 0 & 0.043807 & -1.379516 & -0.789721 \\
\hline 110 & 1 & 0 & 0.079893 & -1.599335 & -1.736488 \\
\hline
\end{tabular}

Cartesian coordinates (in $\AA$ ) and energy of DFT-Pd(II)-catecholate II $\mathrm{E}(\mathrm{RM} 06)=-2884.02036664 \mathrm{au}$ 


\begin{tabular}{|c|c|c|c|c|c|}
\hline \multirow{2}{*}{$\begin{array}{l}\text { Center } \\
\text { number }\end{array}$} & \multirow{2}{*}{$\begin{array}{l}\text { Atomic } \\
\text { number }\end{array}$} & \multirow{2}{*}{$\begin{array}{l}\text { Atomic } \\
\text { type }\end{array}$} & \multicolumn{3}{|c|}{ Coordinates (Angstroms) } \\
\hline & & & $\mathrm{X}$ & $\mathrm{Y}$ & $\mathrm{Z}$ \\
\hline 1 & 46 & 0 & -1.713181 & 0.164817 & -0.083283 \\
\hline 2 & 15 & 0 & -0.264519 & -1.569889 & -0.417342 \\
\hline 3 & 15 & 0 & 0.010573 & 1.660286 & 0.321752 \\
\hline 4 & 6 & 0 & 2.245839 & -0.279652 & -0.648920 \\
\hline 5 & 6 & 0 & 1.267258 & -1.046819 & -1.281127 \\
\hline 6 & 6 & 0 & 1.355084 & -1.304390 & -2.675469 \\
\hline 7 & 1 & 0 & 0.602482 & -1.932055 & -3.163635 \\
\hline 8 & 6 & 0 & 2.380871 & -0.782821 & -3.421808 \\
\hline 9 & 1 & 0 & 2.439073 & -0.988108 & -4.497508 \\
\hline 10 & 6 & 0 & 3.381721 & 0.017152 & -2.823149 \\
\hline 11 & 6 & 0 & 4.434797 & 0.576304 & -3.590659 \\
\hline 12 & 1 & 0 & 4.457637 & 0.378772 & -4.669225 \\
\hline 13 & 6 & 0 & 5.409713 & 1.339708 & -2.995593 \\
\hline 14 & 1 & 0 & 6.223717 & 1.761367 & -3.595451 \\
\hline 15 & 6 & 0 & 5.366512 & 1.576144 & -1.602789 \\
\hline 16 & 1 & 0 & 6.148602 & 2.181602 & -1.131132 \\
\hline 17 & 6 & 0 & 4.353383 & 1.052996 & -0.834648 \\
\hline 18 & 1 & 0 & 4.334583 & 1.254159 & 0.242281 \\
\hline 19 & 6 & 0 & 3.328111 & 0.258004 & -1.416641 \\
\hline 20 & 6 & 0 & 2.215754 & 0.006842 & 0.816400 \\
\hline 21 & 6 & 0 & 1.343754 & 0.948673 & 1.357986 \\
\hline 22 & 6 & 0 & 1.375963 & 1.238607 & 2.746798 \\
\hline 23 & 1 & 0 & 0.713409 & 2.003939 & 3.164063 \\
\hline 24 & 6 & 0 & 2.243731 & 0.576475 & 3.577940 \\
\hline 25 & 1 & 0 & 2.263861 & 0.805681 & 4.650164 \\
\hline 26 & 6 & 0 & 3.132089 & -0.401425 & 3.071758 \\
\hline 27 & 6 & 0 & 4.022055 & -1.100983 & 3.926015 \\
\hline 28 & 1 & 0 & 4.002866 & -0.875896 & 4.999204 \\
\hline 29 & 6 & 0 & 4.894361 & -2.033844 & 3.419976 \\
\hline 30 & 1 & 0 & 5.582147 & -2.565336 & 4.086765 \\
\hline 31 & 6 & 0 & 4.912202 & -2.302229 & 2.032035 \\
\hline 32 & 1 & 0 & 5.616331 & -3.040235 & 1.631717 \\
\hline 33 & 6 & 0 & 4.057731 & -1.642953 & 1.180425 \\
\hline 34 & 1 & 0 & 4.085938 & -1.865442 & 0.107642 \\
\hline 35 & 6 & 0 & 3.137078 & -0.677051 & 1.670659 \\
\hline 36 & 8 & 0 & -3.280155 & -1.140985 & -0.253596 \\
\hline 37 & 8 & 0 & -3.395491 & 1.549436 & 0.047926 \\
\hline 38 & 6 & 0 & -4.603107 & 0.826206 & 0.039654 \\
\hline 39 & 6 & 0 & -4.463047 & -0.560611 & -0.148168 \\
\hline 40 & 6 & 0 & -5.830009 & 1.458940 & 0.160027 \\
\hline 41 & 6 & 0 & -5.655731 & -1.304937 & -0.213807 \\
\hline 42 & 6 & 0 & -6.994742 & 0.694435 & 0.108248 \\
\hline 43 & 6 & 0 & -6.894180 & -0.685020 & -0.084213 \\
\hline 44 & 1 & 0 & -5.569900 & -2.387630 & -0.359711 \\
\hline 45 & 1 & 0 & -7.972411 & 1.176676 & 0.208741 \\
\hline 46 & 1 & 0 & -7.804219 & -1.294146 & -0.132108 \\
\hline 47 & 1 & 0 & -3.415629 & 2.311352 & 0.655979 \\
\hline 48 & 6 & 0 & -0.778788 & 2.998652 & 1.284642 \\
\hline 49 & 6 & 0 & -1.598107 & 2.644548 & 2.371895 \\
\hline 50 & 6 & 0 & -0.677576 & 4.343391 & 0.913525 \\
\hline 51 & 6 & 0 & -2.286372 & 3.623410 & 3.082639 \\
\hline 52 & 1 & 0 & -1.702247 & 1.588692 & 2.662383 \\
\hline 53 & 6 & 0 & -1.372074 & 5.320618 & 1.628162 \\
\hline 54 & 1 & 0 & -0.062284 & 4.636725 & 0.054917 \\
\hline 55 & 6 & 0 & -2.172709 & 4.965044 & 2.710180 \\
\hline 56 & 1 & 0 & -2.917301 & 3.338071 & 3.931826 \\
\hline 57 & 1 & 0 & -1.287048 & 6.371079 & 1.329310 \\
\hline 58 & 6 & 0 & 0.754910 & 2.427055 & -1.142675 \\
\hline 59 & 6 & 0 & 0.152919 & 2.240368 & -2.392032 \\
\hline 60 & 6 & 0 & 1.905505 & 3.218794 & -1.030624 \\
\hline 61 & 6 & 0 & 0.704964 & 2.834739 & -3.524838 \\
\hline 62 & 1 & 0 & -0.749186 & 1.617714 & -2.477067 \\
\hline 63 & 6 & 0 & 2.449572 & 3.814014 & -2.164406 \\
\hline 64 & 1 & 0 & 2.382430 & 3.364186 & -0.051439 \\
\hline 65 & 6 & 0 & 1.852702 & 3.617525 & -3.410697 \\
\hline 66 & 1 & 0 & 0.234759 & 2.685525 & -4.502922 \\
\hline 67 & 1 & 0 & 3.352092 & 4.429420 & -2.076976 \\
\hline 68 & 6 & 0 & -1.096232 & -2.793808 & -1.469970 \\
\hline 69 & 6 & 0 & -1.825887 & -2.349999 & -2.582067 \\
\hline
\end{tabular}




$\begin{array}{lllrrr}70 & 6 & 0 & -1.046604 & -4.159199 & -1.172778 \\ 71 & 6 & 0 & -2.469844 & -3.265832 & -3.404299 \\ 72 & 1 & 0 & -1.902749 & -1.276156 & -2.798421 \\ 73 & 6 & 0 & -1.699704 & -5.073371 & -1.998655 \\ 74 & 1 & 0 & -0.507713 & -4.520690 & -0.289442 \\ 75 & 6 & 0 & -2.406401 & -4.629598 & -3.112861 \\ 76 & 1 & 0 & -3.040778 & -2.912949 & -4.269900 \\ 77 & 1 & 0 & -1.659145 & -6.141947 & -1.761096 \\ 78 & 6 & 0 & 0.161510 & -2.386121 & 1.142842 \\ 79 & 6 & 0 & -0.647917 & -2.184495 & 2.266938 \\ 80 & 6 & 0 & 1.244968 & -3.271450 & 1.208770 \\ 81 & 6 & 0 & -0.361370 & -2.852211 & 3.455345 \\ 82 & 1 & 0 & -1.512460 & -1.509017 & 2.203731 \\ 83 & 6 & 0 & 1.523131 & -3.938620 & 2.397851 \\ 84 & 1 & 0 & 1.876484 & -3.439877 & 0.326174 \\ 85 & 6 & 0 & 0.723280 & -3.725577 & 3.521037 \\ 86 & 1 & 0 & -0.995124 & -2.694520 & 4.334759 \\ 87 & 1 & 0 & 2.375016 & -4.626109 & 2.449120 \\ 88 & 1 & 0 & -5.870896 & 2.548355 & 0.289833 \\ 89 & 1 & 0 & 0.946009 & -4.250884 & 4.456511 \\ 90 & 1 & 0 & 2.286851 & 4.083781 & -4.302265 \\ 91 & 1 & 0 & -2.717805 & 5.735496 & 3.266472 \\ 92 & 1 & 0 & -2.923075 & -5.350497 & -3.756193\end{array}$

Cartesian coordinates (in $\AA$ ) and energy of INT-I-Pd-C(6)-A $\mathrm{E}(\mathrm{UM} 06)=-2961.17180791 \mathrm{au}$

\begin{tabular}{|c|c|c|c|c|c|}
\hline \multirow{2}{*}{$\begin{array}{l}\text { Center } \\
\text { number }\end{array}$} & \multirow{2}{*}{$\begin{array}{l}\text { Atomic } \\
\text { number }\end{array}$} & \multirow{2}{*}{$\begin{array}{l}\text { Atomic } \\
\text { type }\end{array}$} & \multicolumn{3}{|c|}{ Coordinates (Angstroms) } \\
\hline & & & $\mathrm{X}$ & $\mathrm{Y}$ & $\mathrm{Z}$ \\
\hline 1 & 46 & 0 & -0.713114 & 0.314948 & -0.551884 \\
\hline 2 & 15 & 0 & -2.936484 & 0.849512 & -0.616592 \\
\hline 3 & 15 & 0 & -1.463987 & -1.859118 & -0.886764 \\
\hline 4 & 8 & 0 & -0.037304 & 2.347278 & -0.143196 \\
\hline 5 & 8 & 0 & 1.324805 & 0.143822 & -0.987817 \\
\hline 6 & 6 & 0 & 1.948349 & 1.306614 & -0.938795 \\
\hline 7 & 6 & 0 & 1.317105 & 2.484317 & -0.507479 \\
\hline 8 & 6 & 0 & 3.303582 & 1.444336 & -1.301139 \\
\hline 9 & 6 & 0 & 1.962337 & 3.707371 & -0.396131 \\
\hline 10 & 6 & 0 & 3.310914 & 3.823342 & -0.744866 \\
\hline 11 & 1 & 0 & 1.405937 & 4.580298 & -0.025925 \\
\hline 12 & 1 & 0 & 3.826957 & 0.553091 & -1.667975 \\
\hline 13 & 6 & 0 & 4.030062 & 5.136894 & -0.665239 \\
\hline 14 & 1 & 0 & 5.054457 & 5.014896 & -0.267242 \\
\hline 15 & 1 & 0 & 4.130176 & 5.611833 & -1.661170 \\
\hline 16 & 1 & 0 & 3.500434 & 5.855096 & -0.013829 \\
\hline 17 & 6 & 0 & -3.270866 & -1.738411 & -1.387754 \\
\hline 18 & 6 & 0 & -3.659637 & -0.335725 & -1.819529 \\
\hline 19 & 1 & 0 & -3.211614 & -0.075151 & -2.799023 \\
\hline 20 & 1 & 0 & -4.754004 & -0.204984 & -1.920956 \\
\hline 21 & 1 & 0 & -3.868570 & -2.064880 & -0.516415 \\
\hline 22 & 1 & 0 & -3.457945 & -2.469167 & -2.195091 \\
\hline 23 & 6 & 0 & 3.958242 & 2.665468 & -1.200668 \\
\hline 24 & 1 & 0 & 5.016877 & 2.724879 & -1.487727 \\
\hline 25 & 6 & 0 & 2.789918 & -0.847786 & 1.316496 \\
\hline 26 & 6 & 0 & 4.156182 & -0.567316 & 1.068578 \\
\hline 27 & 6 & 0 & 4.550875 & -1.441690 & 0.029613 \\
\hline 28 & 6 & 0 & 1.840914 & -0.162308 & 2.162535 \\
\hline 29 & 6 & 0 & 0.697851 & -0.818949 & 2.667292 \\
\hline 30 & 6 & 0 & 2.023029 & 1.199581 & 2.481410 \\
\hline 31 & 6 & 0 & -0.194356 & -0.146575 & 3.494730 \\
\hline 32 & 1 & 0 & 0.535398 & -1.876026 & 2.428742 \\
\hline 33 & 6 & 0 & 1.115867 & 1.870652 & 3.292871 \\
\hline 34 & 1 & 0 & 2.870173 & 1.746910 & 2.053105 \\
\hline 35 & 6 & 0 & 0.010461 & 1.197793 & 3.815307 \\
\hline 36 & 1 & 0 & -1.056817 & -0.687346 & 3.905694 \\
\hline 37 & 1 & 0 & 1.276598 & 2.930259 & 3.523488 \\
\hline 38 & 1 & 0 & -0.686492 & 1.721498 & 4.481891 \\
\hline
\end{tabular}




\begin{tabular}{|c|c|c|c|c|c|}
\hline 39 & 8 & 0 & 3.529352 & -2.244187 & -0.367030 \\
\hline 40 & 6 & 0 & 2.425807 & -1.940434 & 0.404878 \\
\hline 41 & 8 & 0 & 1.391777 & -2.539016 & 0.257642 \\
\hline 42 & 6 & 0 & 5.823198 & -1.442808 & -0.503977 \\
\hline 43 & 6 & 0 & 5.111814 & 0.318153 & 1.608103 \\
\hline 44 & 6 & 0 & 6.735796 & -0.529947 & 0.031523 \\
\hline 45 & 1 & 0 & 7.757728 & -0.502832 & -0.368123 \\
\hline 46 & 6 & 0 & 6.400066 & 0.348413 & 1.085532 \\
\hline 47 & 1 & 0 & 6.095990 & -2.132628 & -1.308382 \\
\hline 48 & 1 & 0 & 4.852395 & 0.976469 & 2.445473 \\
\hline 49 & 6 & 0 & 7.428126 & 1.305280 & 1.610899 \\
\hline 50 & 1 & 0 & 7.110661 & 1.761310 & 2.564801 \\
\hline 51 & 1 & 0 & 8.400183 & 0.806660 & 1.780521 \\
\hline 52 & 1 & 0 & 7.611926 & 2.130428 & 0.894950 \\
\hline 53 & 1 & 0 & -0.564938 & 3.102740 & -0.461455 \\
\hline 54 & 6 & 0 & -1.540379 & -2.915038 & 0.594512 \\
\hline 55 & 6 & 0 & -0.850501 & -4.129765 & 0.664788 \\
\hline 56 & 6 & 0 & -2.314003 & -2.491266 & 1.684993 \\
\hline 57 & 6 & 0 & -0.951814 & -4.917279 & 1.808981 \\
\hline 58 & 1 & 0 & -0.212373 & -4.452652 & -0.163494 \\
\hline 59 & 6 & 0 & -2.407407 & -3.280939 & 2.826571 \\
\hline 60 & 1 & 0 & -2.848225 & -1.532240 & 1.657027 \\
\hline 61 & 6 & 0 & -1.724783 & -4.495957 & 2.889543 \\
\hline 62 & 1 & 0 & -0.410826 & -5.868469 & 1.857946 \\
\hline 63 & 1 & 0 & -3.016100 & -2.941754 & 3.673112 \\
\hline 64 & 1 & 0 & -1.795652 & -5.118672 & 3.788275 \\
\hline 65 & 6 & 0 & -3.861575 & 0.760116 & 0.934232 \\
\hline 66 & 6 & 0 & -5.170596 & 0.268710 & 1.006334 \\
\hline 67 & 6 & 0 & -3.203204 & 1.179750 & 2.098322 \\
\hline 68 & 6 & 0 & -5.810011 & 0.182668 & 2.241241 \\
\hline 69 & 1 & 0 & -5.701511 & -0.057516 & 0.103198 \\
\hline 70 & 6 & 0 & -3.852907 & 1.101420 & 3.326964 \\
\hline 71 & 1 & 0 & -2.165540 & 1.543612 & 2.045457 \\
\hline 72 & 6 & 0 & -5.151601 & 0.596239 & 3.399181 \\
\hline 73 & 1 & 0 & -6.831246 & -0.209204 & 2.299147 \\
\hline 74 & 1 & 0 & -3.337765 & 1.428154 & 4.237517 \\
\hline 75 & 1 & 0 & -5.657335 & 0.525122 & 4.368484 \\
\hline 76 & 6 & 0 & -3.125947 & 2.529998 & -1.277748 \\
\hline 77 & 6 & 0 & -2.371629 & 2.887789 & -2.408491 \\
\hline 78 & 6 & 0 & -3.980473 & 3.467411 & -0.684562 \\
\hline 79 & 6 & 0 & -2.483756 & 4.167425 & -2.943359 \\
\hline 80 & 1 & 0 & -1.685145 & 2.162605 & -2.869814 \\
\hline 81 & 6 & 0 & -4.086957 & 4.747205 & -1.227405 \\
\hline 82 & 1 & 0 & -4.567462 & 3.199763 & 0.202724 \\
\hline 83 & 6 & 0 & -3.342489 & 5.096488 & -2.352470 \\
\hline 84 & 1 & 0 & -1.896139 & 4.442364 & -3.825944 \\
\hline 85 & 1 & 0 & -4.758731 & 5.478180 & -0.764480 \\
\hline 86 & 1 & 0 & -3.428455 & 6.104430 & -2.772987 \\
\hline 87 & 6 & 0 & -0.644013 & -2.768141 & -2.223091 \\
\hline 88 & 6 & 0 & -1.238085 & -3.944990 & -2.706608 \\
\hline 89 & 6 & 0 & 0.575254 & -2.326869 & -2.748398 \\
\hline 90 & 6 & 0 & -0.619583 & -4.667638 & -3.720676 \\
\hline 91 & 1 & 0 & -2.178777 & -4.319243 & -2.280615 \\
\hline 92 & 6 & 0 & 1.183725 & -3.058792 & -3.767575 \\
\hline 93 & 1 & 0 & 1.053362 & -1.423222 & -2.347674 \\
\hline 94 & 6 & 0 & 0.590523 & -4.220956 & -4.253860 \\
\hline 95 & 1 & 0 & -1.082668 & -5.587441 & -4.094503 \\
\hline 96 & 1 & 0 & 2.139368 & -2.715853 & -4.178582 \\
\hline 97 & 1 & 0 & 1.076426 & -4.791143 & -5.053608 \\
\hline
\end{tabular}

Cartesian coordinates (in $\AA$ ) and energy of INT-I-Pd-C(6)-B $\mathrm{E}(\mathrm{UM} 06)=-2961.16268645 \mathrm{au}$

\begin{tabular}{cccccc}
$\begin{array}{l}\text { Center } \\
\text { number }\end{array}$ & $\begin{array}{c}\text { Atomic } \\
\text { number }\end{array}$ & $\begin{array}{c}\text { Atomic } \\
\text { type }\end{array}$ & X & \multicolumn{3}{c}{ Coordinates (Angstroms) } \\
- & -1 & & Y & Z \\
\hline 1 & 46 & 0 & -0.975595 & 0.254680 & -0.560785 \\
3 & 15 & 0 & -3.262708 & 0.015748 & -0.684477 \\
3 & 15 & 0 & -0.958512 & -2.025084 & -0.733479
\end{tabular}




\begin{tabular}{|c|c|c|c|c|c|}
\hline 4 & 8 & 0 & -0.814857 & 2.389763 & -0.154500 \\
\hline 5 & 8 & 0 & 1.040302 & 0.514957 & -0.857765 \\
\hline 6 & 6 & 0 & 1.385496 & 1.794442 & -0.842310 \\
\hline 7 & 6 & 0 & 0.501323 & 2.810765 & -0.456684 \\
\hline 8 & 6 & 0 & 2.674123 & 2.215800 & -1.221740 \\
\hline 9 & 6 & 0 & 0.868846 & 4.144328 & -0.351029 \\
\hline 10 & 6 & 0 & 2.169553 & 4.539752 & -0.674206 \\
\hline 11 & 1 & 0 & 0.132761 & 4.883837 & -0.005166 \\
\hline 12 & 1 & 0 & 3.370103 & 1.454133 & -1.593102 \\
\hline 13 & 6 & 0 & 2.621073 & 5.959650 & -0.512098 \\
\hline 14 & 1 & 0 & 3.199904 & 6.306660 & -1.387841 \\
\hline 15 & 1 & 0 & 1.769480 & 6.649607 & -0.373904 \\
\hline 16 & 1 & 0 & 3.283312 & 6.070029 & 0.369357 \\
\hline 17 & 6 & 0 & -2.647505 & -2.597156 & -1.296884 \\
\hline 18 & 6 & 0 & -3.496664 & -1.436629 & -1.796877 \\
\hline 19 & 1 & 0 & -3.154129 & -1.094059 & -2.793341 \\
\hline 20 & 1 & 0 & -4.564080 & -1.707544 & -1.904784 \\
\hline 21 & 1 & 0 & -3.131636 & -3.113070 & -0.447020 \\
\hline 22 & 1 & 0 & -2.500948 & -3.354447 & -2.088815 \\
\hline 23 & 6 & 0 & 3.051019 & 3.549627 & -1.131442 \\
\hline 24 & 1 & 0 & 4.074777 & 3.831823 & -1.410665 \\
\hline 25 & 6 & 0 & 4.171312 & 0.507752 & 0.880974 \\
\hline 26 & 6 & 0 & 3.105677 & 0.880956 & 1.743486 \\
\hline 27 & 6 & 0 & 3.382902 & 2.206560 & 2.154682 \\
\hline 28 & 6 & 0 & 4.415744 & -0.732507 & 0.185351 \\
\hline 29 & 6 & 0 & 5.234130 & -0.782310 & -0.964935 \\
\hline 30 & 6 & 0 & 3.850840 & -1.941115 & 0.645743 \\
\hline 31 & 6 & 0 & 5.450619 & -1.983257 & -1.630028 \\
\hline 32 & 1 & 0 & 5.708812 & 0.137047 & -1.321065 \\
\hline 33 & 6 & 0 & 4.076760 & -3.139275 & -0.020142 \\
\hline 34 & 1 & 0 & 3.277365 & -1.937208 & 1.579310 \\
\hline 35 & 6 & 0 & 4.871635 & -3.166196 & -1.167951 \\
\hline 36 & 1 & 0 & 6.092290 & -1.997798 & -2.518682 \\
\hline 37 & 1 & 0 & 3.650924 & -4.072777 & 0.371208 \\
\hline 38 & 1 & 0 & 5.056630 & -4.113333 & -1.688156 \\
\hline 39 & 8 & 0 & 4.533723 & 2.672025 & 1.618805 \\
\hline 40 & 6 & 0 & 5.048205 & 1.690235 & 0.783041 \\
\hline 41 & 8 & 0 & 6.034157 & 1.890335 & 0.131361 \\
\hline 42 & 6 & 0 & 2.531397 & 2.932717 & 2.962559 \\
\hline 43 & 6 & 0 & 1.888399 & 0.296030 & 2.135792 \\
\hline 44 & 6 & 0 & 1.342656 & 2.312193 & 3.355503 \\
\hline 45 & 1 & 0 & 0.640561 & 2.863681 & 3.994065 \\
\hline 46 & 6 & 0 & 1.004747 & 1.008053 & 2.943504 \\
\hline 47 & 1 & 0 & 2.779300 & 3.956932 & 3.257842 \\
\hline 48 & 1 & 0 & 1.606613 & -0.701784 & 1.780366 \\
\hline 49 & 6 & 0 & -0.288721 & 0.380739 & 3.373446 \\
\hline 50 & 1 & 0 & -0.733522 & -0.223808 & 2.555675 \\
\hline 51 & 1 & 0 & -0.150370 & -0.304270 & 4.234282 \\
\hline 52 & 1 & 0 & -1.027117 & 1.144241 & 3.683839 \\
\hline 53 & 1 & 0 & -1.469365 & 3.031203 & -0.482895 \\
\hline 54 & 6 & 0 & -0.693617 & -2.732309 & 0.919099 \\
\hline 55 & 6 & 0 & 0.598737 & -3.131847 & 1.281798 \\
\hline 56 & 6 & 0 & -1.727353 & -2.786347 & 1.866145 \\
\hline 57 & 6 & 0 & 0.849682 & -3.592223 & 2.572513 \\
\hline 58 & 1 & 0 & 1.415938 & -3.078696 & 0.550736 \\
\hline 59 & 6 & 0 & -1.470437 & -3.252461 & 3.151721 \\
\hline 60 & 1 & 0 & -2.745452 & -2.459620 & 1.617337 \\
\hline 61 & 6 & 0 & -0.182662 & -3.655295 & 3.507079 \\
\hline 62 & 1 & 0 & 1.862494 & -3.909327 & 2.848396 \\
\hline 63 & 1 & 0 & -2.285876 & -3.299424 & 3.882237 \\
\hline 64 & 1 & 0 & 0.016934 & -4.022697 & 4.519711 \\
\hline 65 & 6 & 0 & -4.137204 & -0.311026 & 0.866379 \\
\hline 66 & 6 & 0 & -5.209648 & -1.207230 & 0.956757 \\
\hline 67 & 6 & 0 & -3.673235 & 0.341202 & 2.016385 \\
\hline 68 & 6 & 0 & -5.797039 & -1.461692 & 2.194719 \\
\hline 69 & 1 & 0 & -5.594133 & -1.718958 & 0.065399 \\
\hline 70 & 6 & 0 & -4.266422 & 0.087039 & 3.249523 \\
\hline 71 & 1 & 0 & -2.825582 & 1.037522 & 1.945790 \\
\hline 72 & 6 & 0 & -5.322958 & -0.819886 & 3.339155 \\
\hline 73 & 1 & 0 & -6.633053 & -2.166002 & 2.265802 \\
\hline 74 & 1 & 0 & -3.895822 & 0.593986 & 4.147452 \\
\hline 75 & 1 & 0 & -5.785243 & -1.026738 & 4.31 \\
\hline
\end{tabular}




\begin{tabular}{|c|c|c|c|c|c|}
\hline 76 & 6 & 0 & -4.016566 & 1.461019 & -1.476431 \\
\hline 77 & 6 & 0 & -5.190208 & 2.051795 & -0.993177 \\
\hline 78 & 6 & 0 & -3.373054 & 1.997587 & -2.603868 \\
\hline 79 & 6 & 0 & -5.721228 & 3.163742 & -1.644958 \\
\hline 80 & 1 & 0 & -5.693362 & 1.644115 & -0.107502 \\
\hline 81 & 6 & 0 & -3.911716 & 3.105467 & -3.250138 \\
\hline 82 & 1 & 0 & -2.441191 & 1.547543 & -2.976980 \\
\hline 83 & 6 & 0 & -5.086149 & 3.687674 & -2.769792 \\
\hline 84 & 1 & 0 & -6.641068 & 3.624729 & -1.269115 \\
\hline 85 & 1 & 0 & -3.411186 & 3.519866 & -4.131955 \\
\hline 86 & 1 & 0 & -5.508209 & 4.562306 & -3.276796 \\
\hline 87 & 6 & 0 & 0.270039 & -2.773194 & -1.833521 \\
\hline 88 & 6 & 0 & 1.224553 & -1.998637 & -2.501160 \\
\hline 89 & 6 & 0 & 0.251193 & -4.169787 & -1.988108 \\
\hline 90 & 6 & 0 & 2.145803 & -2.627011 & -3.339906 \\
\hline 91 & 1 & 0 & 1.264694 & -0.915243 & -2.333382 \\
\hline 92 & 6 & 0 & 1.175949 & -4.782413 & -2.825319 \\
\hline 93 & 1 & 0 & -0.479314 & -4.786127 & -1.445844 \\
\hline 94 & 6 & 0 & 2.118994 & -4.008521 & -3.506168 \\
\hline 95 & 1 & 0 & 2.896821 & -2.023521 & -3.861396 \\
\hline 96 & 1 & 0 & 1.161290 & -5.870838 & -2.949307 \\
\hline 97 & 1 & 0 & 2.844589 & -4.494633 & -4.168452 \\
\hline
\end{tabular}

Cartesian coordinates (in $\AA$ ) and energy of INT-I-Pd-C(6)-C $\mathrm{E}(\mathrm{UM} 06)=-2961.15185105 \mathrm{au}$

\begin{tabular}{|c|c|c|c|c|c|}
\hline \multirow{2}{*}{$\begin{array}{l}\text { Center } \\
\text { number }\end{array}$} & \multirow{2}{*}{$\begin{array}{l}\text { Atomic } \\
\text { number }\end{array}$} & \multirow{2}{*}{$\begin{array}{l}\text { Atomic } \\
\text { type }\end{array}$} & \multicolumn{3}{|c|}{ Coordinates (Angstroms) } \\
\hline & & & $\mathrm{X}$ & $\mathrm{Y}$ & $\mathrm{Z}$ \\
\hline 1 & 46 & 0 & -0.965534 & -0.410373 & 0.200008 \\
\hline 2 & 15 & 0 & -3.154503 & -1.252113 & 0.271591 \\
\hline 3 & 15 & 0 & -1.944963 & 1.666853 & 0.863175 \\
\hline 4 & 8 & 0 & 0.228096 & -2.549836 & -0.199172 \\
\hline 5 & 8 & 0 & 1.285707 & -0.232322 & 0.634199 \\
\hline 6 & 6 & 0 & 2.008603 & -1.268948 & 0.665070 \\
\hline 7 & 6 & 0 & 1.527898 & -2.548212 & 0.182495 \\
\hline 8 & 6 & 0 & 3.371139 & -1.239343 & 1.114833 \\
\hline 9 & 6 & 0 & 2.353945 & -3.636259 & 0.113296 \\
\hline 10 & 6 & 0 & 3.714445 & -3.554655 & 0.518056 \\
\hline 11 & 1 & 0 & 1.976237 & -4.582691 & -0.298589 \\
\hline 12 & 1 & 0 & 3.714749 & -0.296274 & 1.553910 \\
\hline 13 & 6 & 0 & 4.607308 & -4.731585 & 0.319434 \\
\hline 14 & 1 & 0 & 5.564685 & -4.616587 & 0.855888 \\
\hline 15 & 1 & 0 & 4.129260 & -5.672476 & 0.650847 \\
\hline 16 & 1 & 0 & 4.836622 & -4.848854 & -0.758790 \\
\hline 17 & 6 & 0 & -3.726909 & 1.275118 & 1.246603 \\
\hline 18 & 6 & 0 & -3.928279 & -0.194965 & 1.586353 \\
\hline 19 & 1 & 0 & -3.419828 & -0.460551 & 2.535254 \\
\hline 20 & 1 & 0 & -4.999763 & -0.439982 & 1.718244 \\
\hline 21 & 1 & 0 & -4.299036 & 1.541630 & 0.336208 \\
\hline 22 & 1 & 0 & -4.107691 & 1.932317 & 2.050019 \\
\hline 23 & 6 & 0 & 4.181384 & -2.366294 & 1.080747 \\
\hline 24 & 1 & 0 & 5.219105 & -2.302405 & 1.432135 \\
\hline 25 & 6 & 0 & 4.142243 & 0.004822 & -1.140734 \\
\hline 26 & 6 & 0 & 3.200274 & 1.051350 & -1.177886 \\
\hline 27 & 6 & 0 & 2.034119 & 0.515182 & -1.784202 \\
\hline 28 & 6 & 0 & 5.533395 & 0.000886 & -0.733174 \\
\hline 29 & 6 & 0 & 6.442449 & -0.930339 & -1.274235 \\
\hline 30 & 6 & 0 & 6.004114 & 0.908254 & 0.237718 \\
\hline 31 & 6 & 0 & 7.772989 & -0.934413 & -0.870032 \\
\hline 32 & 1 & 0 & 6.097456 & -1.648672 & -2.022740 \\
\hline 33 & 6 & 0 & 7.329797 & 0.890794 & 0.646202 \\
\hline 34 & 1 & 0 & 5.307303 & 1.606938 & 0.713660 \\
\hline 35 & 6 & 0 & 8.222132 & -0.028576 & 0.089219 \\
\hline 36 & 1 & 0 & 8.469132 & -1.656431 & -1.310826 \\
\hline 37 & 1 & 0 & 7.673299 & 1.595058 & 1.411833 \\
\hline 38 & 1 & 0 & 9.269866 & -0.039663 & 0.409498 \\
\hline 39 & 8 & 0 & 2.185566 & -0.771421 & -2.132277 \\
\hline 40 & 6 & 0 & 3.492902 & -1.155372 & -1.793833 \\
\hline
\end{tabular}




\begin{tabular}{|c|c|c|c|c|c|}
\hline 41 & 8 & 0 & 3.894865 & -2.254366 & -2.042910 \\
\hline 42 & 6 & 0 & 0.912914 & 1.291334 & -2.069963 \\
\hline 43 & 6 & 0 & 3.199658 & 2.416139 & -0.797697 \\
\hline 44 & 6 & 0 & 0.959681 & 2.620686 & -1.693318 \\
\hline 45 & 1 & 0 & 0.102801 & 3.268289 & -1.918752 \\
\hline 46 & 6 & 0 & 2.085444 & 3.196609 & -1.039303 \\
\hline 47 & 1 & 0 & 0.047245 & 0.859245 & -2.584920 \\
\hline 48 & 1 & 0 & 4.089131 & 2.873525 & -0.351867 \\
\hline 49 & 6 & 0 & 2.034562 & 4.638662 & -0.639181 \\
\hline 50 & 1 & 0 & 3.015821 & 5.001910 & -0.287628 \\
\hline 51 & 1 & 0 & 1.712686 & 5.281094 & -1.480398 \\
\hline 52 & 1 & 0 & 1.303276 & 4.794890 & 0.179186 \\
\hline 53 & 1 & 0 & -0.069722 & -3.429443 & -0.489837 \\
\hline 54 & 6 & 0 & -2.090697 & 3.077883 & -0.290975 \\
\hline 55 & 6 & 0 & -1.676876 & 4.372432 & 0.042397 \\
\hline 56 & 6 & 0 & -2.603338 & 2.828140 & -1.573529 \\
\hline 57 & 6 & 0 & -1.781780 & 5.402645 & -0.892653 \\
\hline 58 & 1 & 0 & -1.273790 & 4.584736 & 1.041124 \\
\hline 59 & 6 & 0 & -2.710877 & 3.859881 & -2.500848 \\
\hline 60 & 1 & 0 & -2.919373 & 1.811589 & -1.853093 \\
\hline 61 & 6 & 0 & -2.296294 & 5.150001 & -2.162751 \\
\hline 62 & 1 & 0 & -1.461600 & 6.415366 & -0.621453 \\
\hline 63 & 1 & 0 & -3.118458 & 3.656259 & -3.497492 \\
\hline 64 & 1 & 0 & -2.379154 & 5.962120 & -2.893606 \\
\hline 65 & 6 & 0 & -4.200314 & -1.005545 & -1.203577 \\
\hline 66 & 6 & 0 & -5.596310 & -0.905024 & -1.128960 \\
\hline 67 & 6 & 0 & -3.572676 & -0.910501 & -2.451993 \\
\hline 68 & 6 & 0 & -6.348334 & -0.708799 & -2.284553 \\
\hline 69 & 1 & 0 & -6.109684 & -0.990982 & -0.162052 \\
\hline 70 & 6 & 0 & -4.327225 & -0.720756 & -3.608828 \\
\hline 71 & 1 & 0 & -2.477055 & -0.983490 & -2.512214 \\
\hline 72 & 6 & 0 & -5.714460 & -0.616798 & -3.524143 \\
\hline 73 & 1 & 0 & -7.439163 & -0.629922 & -2.218090 \\
\hline 74 & 1 & 0 & -3.828637 & -0.653132 & -4.582213 \\
\hline 75 & 1 & 0 & -6.308961 & -0.463683 & -4.431772 \\
\hline 76 & 6 & 0 & -3.424069 & -2.966860 & 0.827109 \\
\hline 77 & 6 & 0 & -2.694350 & -3.405388 & 1.943500 \\
\hline 78 & 6 & 0 & -4.270239 & -3.863063 & 0.163081 \\
\hline 79 & 6 & 0 & -2.836008 & -4.709531 & 2.406567 \\
\hline 80 & 1 & 0 & -2.001794 & -2.718098 & 2.450932 \\
\hline 81 & 6 & 0 & -4.399204 & -5.172536 & 0.624791 \\
\hline 82 & 1 & 0 & -4.835364 & -3.541072 & -0.720334 \\
\hline 83 & 6 & 0 & -3.689362 & -5.595464 & 1.746633 \\
\hline 84 & 1 & 0 & -2.271681 & -5.040512 & 3.285674 \\
\hline 85 & 1 & 0 & -5.065395 & -5.868022 & 0.102051 \\
\hline 86 & 1 & 0 & -3.797012 & -6.624309 & 2.107837 \\
\hline 87 & 6 & 0 & -1.182695 & 2.378814 & 2.357462 \\
\hline 88 & 6 & 0 & -1.902949 & 3.021101 & 3.371718 \\
\hline 89 & 6 & 0 & 0.218316 & 2.341346 & 2.420016 \\
\hline 90 & 6 & 0 & -1.231099 & 3.619809 & 4.435516 \\
\hline 91 & 1 & 0 & -2.998026 & 3.076456 & 3.337681 \\
\hline 92 & 6 & 0 & 0.884304 & 2.955218 & 3.478743 \\
\hline 93 & 1 & 0 & 0.789692 & 1.815656 & 1.641180 \\
\hline 94 & 6 & 0 & 0.162037 & 3.591963 & 4.487185 \\
\hline 95 & 1 & 0 & -1.801280 & 4.118336 & 5.227369 \\
\hline 96 & 1 & 0 & 1.979102 & 2.925146 & 3.522058 \\
\hline 97 & 1 & 0 & 0.687398 & 4.067241 & 5.323236 \\
\hline
\end{tabular}

Cartesian coordinates (in $\AA$ ) and energy of INT-I-Pd-C(6)-D $\mathrm{E}(\mathrm{UM} 06)=-2961.15734455 \mathrm{au}$

\begin{tabular}{cccccc}
$\begin{array}{l}\text { Center } \\
\text { number }\end{array}$ & $\begin{array}{c}\text { Atomic } \\
\text { number }\end{array}$ & $\begin{array}{c}\text { Atomic } \\
\text { type }\end{array}$ & \multicolumn{3}{c}{ Coordinates (Angstroms) } \\
\hline 1 & 46 & 0 & Y & $Z$ \\
2 & 15 & 0 & 2.781849 & -0.564251 & 0.223366 \\
3 & 15 & 0 & 1.269111 & 2.263124 & 0.021373 \\
4 & 8 & 0 & -0.166906 & -2.372772 & 0.475767 \\
5 & 8 & 0 & -1.387672 & -0.235843 & 1.561529 \\
6 & 6 & 0 & -2.010316 & -1.327447 & 1.515192
\end{tabular}




\begin{tabular}{|c|c|c|c|c|c|}
\hline 7 & 6 & 0 & -1.415442 & -2.528640 & 0.959276 \\
\hline 8 & 6 & 0 & -3.353510 & -1.494401 & 2.019973 \\
\hline 9 & 6 & 0 & -2.067879 & -3.749946 & 0.962197 \\
\hline 10 & 6 & 0 & -3.367670 & -3.866348 & 1.457490 \\
\hline 11 & 1 & 0 & -1.566341 & -4.629850 & 0.536324 \\
\hline 12 & 6 & 0 & -4.107631 & -5.157559 & 1.422465 \\
\hline 13 & 1 & 0 & -4.531173 & -5.405262 & 2.414239 \\
\hline 14 & 1 & 0 & -4.958179 & -5.084322 & 0.716936 \\
\hline 15 & 1 & 0 & -3.468872 & -5.994928 & 1.094618 \\
\hline 16 & 6 & 0 & 3.081381 & 2.173020 & 0.42279 \\
\hline 17 & 6 & 0 & 3.743373 & 0.956650 & -0.21507 \\
\hline 18 & 1 & 0 & 3.736569 & 1.041024 & -1.319067 \\
\hline 19 & 1 & 0 & 4.798721 & 0.853168 & 0.10541 \\
\hline 20 & 1 & 0 & 3.129513 & 2.104101 & 1.5288 \\
\hline 21 & 1 & 0 & 3.587356 & 3.118059 & 0.14334 \\
\hline 22 & 6 & 0 & -3.988110 & -2.706958 & 2.006255 \\
\hline 23 & 1 & 0 & -5.010018 & -2.795378 & 2.395340 \\
\hline 24 & 1 & 0 & 0.050630 & -3.049654 & -0.195165 \\
\hline 25 & 6 & 0 & 0.579912 & 3.436524 & 1.230156 \\
\hline 26 & 6 & 0 & -0.169859 & 2.925537 & 2.297823 \\
\hline 27 & 6 & 0 & 0.805576 & 4.816176 & 1.128154 \\
\hline 28 & 6 & 0 & -0.678087 & 3.792886 & 3.264329 \\
\hline 29 & 1 & 0 & -0.380546 & 1.847612 & 2.358162 \\
\hline 30 & 6 & 0 & 0.287233 & 5.675976 & 2.092329 \\
\hline 31 & 1 & 0 & 1.385307 & 5.221903 & 0.28808 \\
\hline 32 & 6 & 0 & -0.451277 & 5.164016 & 3.160978 \\
\hline 33 & 1 & 0 & -1.264893 & 3.392986 & 4.098730 \\
\hline 34 & 1 & 0 & 0.462265 & 6.754677 & 2.012025 \\
\hline 35 & 1 & 0 & -0.856354 & 5.844641 & 3.918400 \\
\hline 36 & 6 & 0 & 3.308937 & -0.960835 & 1.926026 \\
\hline 37 & 6 & 0 & 4.634898 & -1.322518 & 2.204011 \\
\hline 38 & 6 & 0 & 2.378511 & -0.879163 & 2.968892 \\
\hline 39 & 6 & 0 & 5.024594 & -1.589835 & 3.512547 \\
\hline 40 & 1 & 0 & 5.367840 & -1.404889 & 1.389657 \\
\hline 41 & 6 & 0 & 2.774689 & -1.146667 & 4.279423 \\
\hline 42 & 1 & 0 & 1.333920 & -0.610680 & 2.748137 \\
\hline 43 & 6 & 0 & 4.094684 & -1.499820 & 4.550111 \\
\hline 44 & 1 & 0 & 6.061301 & -1.872779 & 3.726524 \\
\hline 45 & 1 & 0 & 2.044087 & -1.082190 & 5.093290 \\
\hline 46 & 1 & 0 & 4.404188 & -1.711676 & 5.579732 \\
\hline 47 & 6 & 0 & 3.420628 & -1.919478 & -0.807723 \\
\hline 48 & 6 & 0 & 4.050339 & -1.709594 & -2.040600 \\
\hline 49 & 6 & 0 & 3.141982 & -3.234187 & -0.398642 \\
\hline 50 & 6 & 0 & 4.403565 & -2.793645 & -2.844036 \\
\hline 51 & 1 & 0 & 4.281777 & -0.695215 & -2.388061 \\
\hline 52 & 6 & 0 & 3.489196 & -4.312146 & -1.206768 \\
\hline 53 & 1 & 0 & 2.661893 & -3.412593 & 0.574199 \\
\hline 54 & 6 & 0 & 4.119118 & -4.094245 & -2.433705 \\
\hline 55 & 1 & 0 & 4.911404 & -2.617693 & -3.799187 \\
\hline 56 & 1 & 0 & 3.278527 & -5.334359 & -0.872186 \\
\hline 57 & 1 & 0 & 4.398828 & -4.943740 & -3.066476 \\
\hline 58 & 6 & 0 & 1.165447 & 3.095827 & -1.600971 \\
\hline 59 & 6 & 0 & 2.271859 & 3.268449 & -2.443091 \\
\hline 60 & 6 & 0 & -0.101262 & 3.489320 & -2.062084 \\
\hline 61 & 6 & 0 & 2.111368 & 3.806627 & -3.720206 \\
\hline 62 & 1 & 0 & 3.281052 & 2.999158 & -2.110129 \\
\hline 63 & 6 & 0 & -0.258426 & 4.027817 & -3.334623 \\
\hline 64 & 1 & 0 & -0.977727 & 3.391744 & -1.407137 \\
\hline 65 & 6 & 0 & 0.847949 & 4.179442 & -4.171683 \\
\hline 66 & 1 & 0 & 2.987161 & 3.941332 & -4.364847 \\
\hline 67 & 1 & 0 & -1.253135 & 4.335131 & -3.675916 \\
\hline 68 & 1 & 0 & 0.724562 & 4.599346 & -5.175906 \\
\hline 69 & 6 & 0 & -2.929070 & -1.023258 & -1.161743 \\
\hline 70 & 6 & 0 & -3.468772 & 0.228885 & -0.721885 \\
\hline 71 & 6 & 0 & -4.843786 & -0.000501 & -0.495595 \\
\hline 72 & 6 & 0 & -4.049916 & -1.988537 & -1.058855 \\
\hline 73 & 8 & 0 & -5.185824 & -1.295327 & -0.709648 \\
\hline 74 & 8 & 0 & -4.074636 & -3.183388 & -1.181996 \\
\hline 75 & 6 & 0 & -1.700157 & -1.294474 & -1.859111 \\
\hline 76 & 6 & 0 & -0.806036 & -0.244400 & -2.186963 \\
\hline 77 & 6 & 0 & -1.347485 & -2.606879 & -2.272418 \\
\hline 7 & 6 & 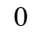 & 0.378457 & -0.496063 & -2.88 \\
\hline
\end{tabular}




$\begin{array}{rrrrrr}79 & 1 & 0 & -1.099980 & 0.795242 & -2.016298 \\ 80 & 6 & 0 & -0.165461 & -2.844017 & -2.955828 \\ 81 & 1 & 0 & -2.038991 & -3.428863 & -2.059901 \\ 82 & 6 & 0 & 0.710500 & -1.792763 & -3.262696 \\ 83 & 1 & 0 & 1.026765 & 0.349356 & -3.156147 \\ 84 & 1 & 0 & 0.078089 & -3.863803 & -3.277041 \\ 85 & 1 & 0 & 1.636090 & -1.991855 & -3.814408 \\ 86 & 6 & 0 & -2.942671 & 1.491585 & -0.392437 \\ 87 & 6 & 0 & -5.704323 & 0.972386 & -0.037732 \\ 88 & 6 & 0 & -5.153509 & 2.227925 & 0.236030 \\ 89 & 1 & 0 & -5.809911 & 3.025917 & 0.605650 \\ 90 & 6 & 0 & -3.783695 & 2.501523 & 0.079154 \\ 91 & 1 & 0 & -1.866169 & 1.682375 & -0.449566 \\ 92 & 1 & 0 & -6.764912 & 0.754541 & 0.118628 \\ 93 & 6 & 0 & -3.249340 & 3.867353 & 0.396214 \\ 94 & 1 & 0 & -3.431669 & 4.574706 & -0.437101 \\ 95 & 1 & 0 & -3.733808 & 4.297293 & 1.292038 \\ 96 & 1 & 0 & -2.160450 & 3.848691 & 0.585335 \\ 97 & 1 & 0 & -3.837417 & -0.594661 & 2.420868\end{array}$

Cartesian coordinates (in $\AA$ ) and energy of INT-I-Pd-C(6)-E $\mathrm{E}(\mathrm{UM} 06)=-2961.17667643 \mathrm{au}$

\begin{tabular}{|c|c|c|c|c|c|}
\hline \multirow{2}{*}{$\begin{array}{l}\text { Center } \\
\text { number }\end{array}$} & \multirow{2}{*}{$\begin{array}{l}\text { Atomic } \\
\text { number }\end{array}$} & \multirow{2}{*}{$\begin{array}{l}\text { Atomic } \\
\text { type }\end{array}$} & \multicolumn{3}{|c|}{ Coordinates (Angstroms) } \\
\hline & & & $X$ & $\mathrm{Y}$ & $\mathrm{Z}$ \\
\hline 1 & 46 & 0 & -1.006530 & 0.063187 & -0.385843 \\
\hline 2 & 15 & 0 & -2.791194 & -1.158639 & 0.386092 \\
\hline 3 & 15 & 0 & -2.132021 & 1.857527 & 0.461435 \\
\hline 4 & 8 & 0 & 0.203230 & -1.522561 & -1.287549 \\
\hline 5 & 8 & 0 & 0.543176 & 1.203863 & -1.120404 \\
\hline 6 & 6 & 0 & 1.463809 & 0.469900 & -1.719068 \\
\hline 7 & 6 & 0 & 1.361517 & -0.926409 & -1.837549 \\
\hline 8 & 6 & 0 & 2.634633 & 1.048236 & -2.248518 \\
\hline 9 & 6 & 0 & 2.338910 & -1.711408 & -2.429510 \\
\hline 10 & 6 & 0 & 3.514133 & -1.129786 & -2.911995 \\
\hline 11 & 1 & 0 & 2.189198 & -2.799084 & -2.484213 \\
\hline 12 & 6 & 0 & 4.606860 & -1.959045 & -3.517807 \\
\hline 13 & 1 & 0 & 5.604382 & -1.618549 & -3.179336 \\
\hline 14 & 1 & 0 & 4.505309 & -3.027202 & -3.250400 \\
\hline 15 & 1 & 0 & 4.610369 & -1.899872 & -4.624098 \\
\hline 16 & 6 & 0 & -3.807924 & 1.283065 & 1.019573 \\
\hline 17 & 6 & 0 & -3.719286 & -0.119650 & 1.599893 \\
\hline 18 & 1 & 0 & -3.167613 & -0.108643 & 2.558543 \\
\hline 19 & 1 & 0 & -4.719668 & -0.553702 & 1.790923 \\
\hline 20 & 1 & 0 & -4.464426 & 1.295194 & 0.126140 \\
\hline 21 & 1 & 0 & -4.216625 & 2.002758 & 1.754040 \\
\hline 22 & 6 & 0 & 3.632956 & 0.264741 & -2.810133 \\
\hline 23 & 1 & 0 & 4.551492 & 0.750880 & -3.167479 \\
\hline 24 & 1 & 0 & 0.469749 & -2.218671 & -0.648423 \\
\hline 25 & 6 & 0 & -2.402594 & 3.227156 & -0.684031 \\
\hline 26 & 6 & 0 & -1.365498 & 3.589326 & -1.555059 \\
\hline 27 & 6 & 0 & -3.597143 & 3.961371 & -0.659592 \\
\hline 28 & 6 & 0 & -1.535790 & 4.682524 & -2.401061 \\
\hline 29 & 1 & 0 & -0.436211 & 3.002035 & -1.566348 \\
\hline 30 & 6 & 0 & -3.753232 & 5.052649 & -1.509826 \\
\hline 31 & 1 & 0 & -4.411488 & 3.698702 & 0.026396 \\
\hline 32 & 6 & 0 & -2.724169 & 5.411528 & -2.380109 \\
\hline 33 & 1 & 0 & -0.729647 & 4.966240 & -3.086398 \\
\hline 34 & 1 & 0 & -4.685397 & 5.627770 & -1.490530 \\
\hline 35 & 1 & 0 & -2.851573 & 6.270114 & -3.048913 \\
\hline 36 & 6 & 0 & -3.978579 & -1.530984 & -0.936830 \\
\hline 37 & 6 & 0 & -5.090559 & -2.342651 & -0.668357 \\
\hline 38 & 6 & 0 & -3.803963 & -0.981942 & -2.212579 \\
\hline 39 & 6 & 0 & -6.021585 & -2.594666 & -1.670271 \\
\hline 40 & 1 & 0 & -5.226055 & -2.788491 & 0.326633 \\
\hline 41 & 6 & 0 & -4.741174 & -1.239690 & -3.212648 \\
\hline 42 & 1 & 0 & -2.926221 & -0.355598 & -2.426435 \\
\hline 43 & 6 & 0 & -5.846463 & -2.042570 & -2.940937 \\
\hline 44 & 1 & 0 & -6.889728 & -3.229285 & -1.461217 \\
\hline
\end{tabular}




\begin{tabular}{|c|c|c|c|c|c|}
\hline 45 & 1 & 0 & -4.602041 & -0.812489 & -4.211524 \\
\hline 46 & 1 & 0 & -6.580569 & -2.245587 & -3.728543 \\
\hline 47 & 6 & 0 & -2.357076 & -2.737631 & 1.161405 \\
\hline 48 & 6 & 0 & -2.246328 & -2.882527 & 2.549759 \\
\hline 49 & 6 & 0 & -2.023054 & -3.810643 & 0.320906 \\
\hline 50 & 6 & 0 & -1.822784 & -4.095214 & 3.091307 \\
\hline 51 & 1 & 0 & -2.494336 & -2.055509 & 3.226104 \\
\hline 52 & 6 & 0 & -1.598514 & -5.017684 & 0.868565 \\
\hline 53 & 1 & 0 & -2.106674 & -3.703328 & -0.769462 \\
\hline 54 & 6 & 0 & -1.499097 & -5.161292 & 2.253460 \\
\hline 55 & 1 & 0 & -1.748736 & -4.207595 & 4.178371 \\
\hline 56 & 1 & 0 & -1.350520 & -5.856740 & 0.208897 \\
\hline 57 & 1 & 0 & -1.170802 & -6.113990 & 2.683501 \\
\hline 58 & 6 & 0 & -1.277026 & 2.489506 & 1.924568 \\
\hline 59 & 6 & 0 & -0.851653 & 1.588118 & 2.910129 \\
\hline 60 & 6 & 0 & -1.042101 & 3.860419 & 2.082065 \\
\hline 61 & 6 & 0 & -0.229334 & 2.058470 & 4.059307 \\
\hline 62 & 1 & 0 & -0.959195 & 0.505831 & 2.759174 \\
\hline 63 & 6 & 0 & -0.405880 & 4.321819 & 3.231615 \\
\hline 64 & 1 & 0 & -1.351455 & 4.572026 & 1.306930 \\
\hline 65 & 6 & 0 & -0.006856 & 3.425317 & 4.220380 \\
\hline 66 & 1 & 0 & 0.111194 & 1.350589 & 4.822816 \\
\hline 67 & 1 & 0 & -0.217211 & 5.394022 & 3.352534 \\
\hline 68 & 1 & 0 & 0.498078 & 3.794308 & 5.119899 \\
\hline 69 & 6 & 0 & 3.551191 & 0.209057 & 0.904779 \\
\hline 70 & 6 & 0 & 3.307325 & -1.196437 & 0.840524 \\
\hline 71 & 6 & 0 & 2.014231 & -1.393972 & 1.373282 \\
\hline 72 & 6 & 0 & 2.342025 & 0.809242 & 1.493857 \\
\hline 73 & 8 & 0 & 1.444746 & -0.232943 & 1.773834 \\
\hline 74 & 8 & 0 & 2.053005 & 1.940042 & 1.761213 \\
\hline 75 & 6 & 0 & 4.699008 & 0.971865 & 0.488317 \\
\hline 76 & 6 & 0 & 5.952246 & 0.352517 & 0.287255 \\
\hline 77 & 6 & 0 & 4.594207 & 2.357525 & 0.228918 \\
\hline 78 & 6 & 0 & 7.037822 & 1.072785 & -0.190485 \\
\hline 79 & 1 & 0 & 6.086589 & -0.700904 & 0.550590 \\
\hline 80 & 6 & 0 & 5.682287 & 3.069941 & -0.255270 \\
\hline 81 & 1 & 0 & 3.636522 & 2.859633 & 0.394883 \\
\hline 82 & 6 & 0 & 6.905509 & 2.433166 & -0.476965 \\
\hline 83 & 1 & 0 & 8.002964 & 0.573182 & -0.330251 \\
\hline 84 & 1 & 0 & 5.577821 & 4.140572 & -0.464401 \\
\hline 85 & 1 & 0 & 7.761388 & 3.000895 & -0.859015 \\
\hline 86 & 6 & 0 & 3.987445 & -2.312003 & 0.316464 \\
\hline 87 & 6 & 0 & 1.400165 & -2.632739 & 1.418619 \\
\hline 88 & 6 & 0 & 2.111866 & -3.721659 & 0.897810 \\
\hline 89 & 1 & 0 & 1.657439 & -4.721049 & 0.931555 \\
\hline 90 & 6 & 0 & 3.396449 & -3.575185 & 0.340714 \\
\hline 91 & 1 & 0 & 4.970890 & -2.200276 & -0.152352 \\
\hline 92 & 1 & 0 & 0.404327 & -2.749280 & 1.865827 \\
\hline 93 & 6 & 0 & 4.093294 & -4.762947 & -0.254155 \\
\hline 94 & 1 & 0 & 5.158178 & -4.552840 & -0.455395 \\
\hline 95 & 1 & 0 & 4.044044 & -5.642399 & 0.414241 \\
\hline 96 & 1 & 0 & 3.630592 & -5.064925 & -1.214923 \\
\hline 97 & 1 & 0 & 2.749649 & 2.135065 & -2.163556 \\
\hline
\end{tabular}

Cartesian coordinates (in $\AA$ ) and energy of INT-I-Pd-C(6)-F $\mathrm{E}(\mathrm{UM} 06)=-2961.18710618 \mathrm{au}$

\begin{tabular}{cccccc}
$\begin{array}{c}\text { Center } \\
\text { number }\end{array}$ & $\begin{array}{c}\text { Atomic } \\
\text { number }\end{array}$ & $\begin{array}{c}\text { Atomic } \\
\text { type }\end{array}$ & \multicolumn{3}{c}{ Coordinates (Angstroms) } \\
\hline 1 & 46 & 0 & -0.951640 & -0.209184 & -0.400957 \\
2 & 15 & 0 & -2.955227 & -1.179334 & 0.210829 \\
3 & 15 & 0 & -2.091359 & 1.743233 & -0.241378 \\
4 & 8 & 0 & 0.378483 & -1.962186 & -0.671070 \\
5 & 8 & 0 & 0.667334 & 0.722481 & -1.262821 \\
6 & 6 & 0 & 1.602187 & -0.124506 & -1.621545 \\
7 & 6 & 0 & 1.536404 & -1.505235 & -1.338923 \\
8 & 6 & 0 & 2.750506 & 0.305510 & -2.322335 \\
9 & 6 & 0 & 2.538403 & -2.396726 & -1.688563 \\
10 & 6 & 0 & 3.687278 & -1.949703 & -2.348775
\end{tabular}




\begin{tabular}{|c|c|c|c|c|c|}
\hline 11 & 1 & 0 & 2.421500 & -3.457911 & -1.427186 \\
\hline 12 & 6 & 0 & 4.781926 & -2.901990 & -2.721422 \\
\hline 13 & 1 & 0 & 5.714960 & -2.364831 & -2.971910 \\
\hline 14 & 1 & 0 & 5.008910 & -3.601925 & -1.893853 \\
\hline 15 & 1 & 0 & 4.514393 & -3.518254 & -3.602595 \\
\hline 16 & 6 & 0 & -3.894079 & 1.347107 & -0.331569 \\
\hline 17 & 6 & 0 & -4.149969 & 0.175602 & 0.607474 \\
\hline 18 & 1 & 0 & -3.953024 & 0.458630 & 1.660791 \\
\hline 19 & 1 & 0 & -5.195433 & -0.183469 & 0.567736 \\
\hline 20 & 1 & 0 & -4.137916 & 1.079102 & -1.378804 \\
\hline 21 & 1 & 0 & -4.486294 & 2.242293 & -0.057484 \\
\hline 22 & 6 & 0 & 3.759567 & -0.582635 & -2.662868 \\
\hline 23 & 1 & 0 & 4.643968 & -0.208771 & -3.195893 \\
\hline 24 & 1 & 0 & 0.628673 & -2.348240 & 0.194364 \\
\hline 25 & 6 & 0 & -1.664373 & 2.972400 & -1.481294 \\
\hline 26 & 6 & 0 & -0.359524 & 3.487974 & -1.443306 \\
\hline 27 & 6 & 0 & -2.567559 & 3.401902 & -2.461438 \\
\hline 28 & 6 & 0 & 0.026334 & 4.441037 & -2.378679 \\
\hline 29 & 1 & 0 & 0.354807 & 3.131256 & -0.690820 \\
\hline 30 & 6 & 0 & -2.169432 & 4.358234 & -3.392595 \\
\hline 31 & 1 & 0 & -3.588303 & 3.003233 & -2.504988 \\
\hline 32 & 6 & 0 & -0.875446 & 4.875666 & -3.350962 \\
\hline 33 & 1 & 0 & 1.045342 & 4.843505 & -2.350357 \\
\hline 34 & 1 & 0 & -2.876418 & 4.701920 & -4.155401 \\
\hline 35 & 1 & 0 & -0.566089 & 5.626062 & -4.087100 \\
\hline 36 & 6 & 0 & -3.612278 & -2.056743 & -1.241071 \\
\hline 37 & 6 & 0 & -4.909654 & -1.866803 & -1.731378 \\
\hline 38 & 6 & 0 & -2.758317 & -2.972099 & -1.874270 \\
\hline 39 & 6 & 0 & -5.348383 & -2.588093 & -2.840695 \\
\hline 40 & 1 & 0 & -5.598397 & -1.158648 & -1.254430 \\
\hline 41 & 6 & 0 & -3.204282 & -3.691997 & -2.977936 \\
\hline 42 & 1 & 0 & -1.733205 & -3.115006 & -1.505547 \\
\hline 43 & 6 & 0 & -4.498206 & -3.499820 & -3.462598 \\
\hline 44 & 1 & 0 & -6.365046 & -2.436096 & -3.219488 \\
\hline 45 & 1 & 0 & -2.533074 & -4.405220 & -3.468450 \\
\hline 46 & 1 & 0 & -4.845910 & -4.064340 & -4.334748 \\
\hline 47 & 6 & 0 & -2.980568 & -2.355229 & 1.589387 \\
\hline 48 & 6 & 0 & -1.897946 & -2.422846 & 2.474830 \\
\hline 49 & 6 & 0 & -4.098713 & -3.180950 & 1.773861 \\
\hline 50 & 6 & 0 & -1.944188 & -3.313792 & 3.547124 \\
\hline 51 & 1 & 0 & -1.009613 & -1.791501 & 2.327388 \\
\hline 52 & 6 & 0 & -4.137072 & -4.060947 & 2.849944 \\
\hline 53 & 1 & 0 & -4.940390 & -3.143481 & 1.069077 \\
\hline 54 & 6 & 0 & -3.059735 & -4.126792 & 3.735470 \\
\hline 55 & 1 & 0 & -1.096437 & -3.373231 & 4.238344 \\
\hline 56 & 1 & 0 & -5.010309 & -4.706065 & 2.996318 \\
\hline 57 & 1 & 0 & -3.090281 & -4.825921 & 4.578653 \\
\hline 58 & 6 & 0 & -1.885436 & 2.527561 & 1.385348 \\
\hline 59 & 6 & 0 & -1.365709 & 1.818391 & 2.472807 \\
\hline 60 & 6 & 0 & -2.314592 & 3.852981 & 1.549257 \\
\hline 61 & 6 & 0 & -1.284607 & 2.432893 & 3.722229 \\
\hline 62 & 1 & 0 & -0.988073 & 0.796415 & 2.336391 \\
\hline 63 & 6 & 0 & -2.228915 & 4.458887 & 2.798817 \\
\hline 64 & 1 & 0 & -2.710335 & 4.418998 & 0.695158 \\
\hline 65 & 6 & 0 & -1.713980 & 3.748501 & 3.884999 \\
\hline 66 & 1 & 0 & -0.871749 & 1.879075 & 4.572924 \\
\hline 67 & 1 & 0 & -2.563204 & 5.494390 & 2.926248 \\
\hline 68 & 1 & 0 & -1.645366 & 4.228698 & 4.867581 \\
\hline 69 & 6 & 0 & 3.096653 & 0.193176 & 1.180993 \\
\hline 70 & 6 & 0 & 4.248870 & -0.603548 & 0.930699 \\
\hline 71 & 6 & 0 & 3.867280 & -1.940165 & 1.172952 \\
\hline 72 & 6 & 0 & 2.048670 & -0.736174 & 1.583325 \\
\hline 73 & 8 & 0 & 2.555565 & -2.021966 & 1.543987 \\
\hline 74 & 8 & 0 & 0.879664 & -0.573640 & 1.873241 \\
\hline 75 & 6 & 0 & 2.892047 & 1.619678 & 1.062551 \\
\hline 76 & 6 & 0 & 1.912958 & 2.273756 & 1.837397 \\
\hline 77 & 6 & 0 & 3.657234 & 2.395469 & 0.168561 \\
\hline 78 & 6 & 0 & 1.714157 & 3.644821 & 1.729132 \\
\hline 79 & 1 & 0 & 1.322903 & 1.693896 & 2.552911 \\
\hline 80 & 6 & 0 & 3.449134 & 3.764431 & 0.055286 \\
\hline 81 & 1 & 0 & 4.395349 & 1.904727 & -0.474824 \\
\hline 82 & 6 & 0 & 2.479294 & 4.397600 & 0.836957 \\
\hline
\end{tabular}




$\begin{array}{rrrrrr}83 & 1 & 0 & 0.956482 & 4.131065 & 2.357010 \\ 84 & 1 & 0 & 4.051851 & 4.347670 & -0.650463 \\ 85 & 1 & 0 & 2.324688 & 5.479690 & 0.753277 \\ 86 & 6 & 0 & 5.584581 & -0.345580 & 0.561262 \\ 87 & 6 & 0 & 4.735310 & -3.006938 & 1.069951 \\ 88 & 6 & 0 & 6.046331 & -2.716828 & 0.687808 \\ 89 & 1 & 0 & 6.769467 & -3.537544 & 0.595607 \\ 90 & 6 & 0 & 6.483376 & -1.399542 & 0.428063 \\ 91 & 1 & 0 & 5.930174 & 0.681822 & 0.398742 \\ 92 & 1 & 0 & 4.404744 & -4.028043 & 1.285179 \\ 93 & 6 & 0 & 7.902814 & -1.162118 & 0.007668 \\ 94 & 1 & 0 & 8.616663 & -1.673275 & 0.679751 \\ 95 & 1 & 0 & 8.088376 & -1.552180 & -1.012263 \\ 96 & 1 & 0 & 8.153761 & -0.087294 & 0.002367 \\ 97 & 1 & 0 & 2.813590 & 1.370587 & -2.575234\end{array}$

Cartesian coordinates (in Å) and energy of TS-Pd-C(6)-A $\mathrm{E}(\mathrm{UM} 06)=-2961.14348841 \mathrm{au}$

\begin{tabular}{|c|c|c|c|c|c|}
\hline \multirow{2}{*}{$\begin{array}{l}\text { Center } \\
\text { number }\end{array}$} & \multirow{2}{*}{$\begin{array}{l}\text { Atomic } \\
\text { number }\end{array}$} & \multirow{2}{*}{$\begin{array}{l}\text { Atomic } \\
\text { type }\end{array}$} & \multicolumn{3}{|c|}{ Coordinates (Angstroms) } \\
\hline & & & $\mathrm{X}$ & $\mathrm{Y}$ & $\mathrm{Z}$ \\
\hline 1 & 46 & 0 & 1.305161 & -0.101373 & -0.827006 \\
\hline 2 & 15 & 0 & 3.572085 & -0.324615 & -0.356770 \\
\hline 3 & 15 & 0 & 1.513515 & 2.026375 & 0.200838 \\
\hline 4 & 8 & 0 & 0.364742 & -2.479912 & -0.972777 \\
\hline 5 & 8 & 0 & -0.662611 & -0.180756 & -1.862439 \\
\hline 6 & 6 & 0 & -1.448143 & -1.136167 & -1.666932 \\
\hline 7 & 6 & 0 & -0.961101 & -2.442121 & -1.249804 \\
\hline 8 & 6 & 0 & -2.882396 & -1.014455 & -1.839776 \\
\hline 9 & 6 & 0 & -1.795330 & -3.516511 & -1.194164 \\
\hline 10 & 6 & 0 & -3.192818 & -3.402490 & -1.508304 \\
\hline 11 & 1 & 0 & -1.399475 & -4.498852 & -0.896360 \\
\hline 12 & 1 & 0 & -3.211967 & -0.106275 & -2.360539 \\
\hline 13 & 6 & 0 & -4.040626 & -4.629762 & -1.434876 \\
\hline 14 & 1 & 0 & -5.097278 & -4.412333 & -1.668586 \\
\hline 15 & 1 & 0 & -3.689142 & -5.407828 & -2.140191 \\
\hline 16 & 1 & 0 & -3.996391 & -5.080933 & -0.423092 \\
\hline 17 & 6 & 0 & 3.335184 & 2.284785 & 0.530253 \\
\hline 18 & 6 & 0 & 4.200724 & 1.417455 & -0.369502 \\
\hline 19 & 1 & 0 & 4.140711 & 1.749928 & -1.425591 \\
\hline 20 & 1 & 0 & 5.269355 & 1.459983 & -0.083025 \\
\hline 21 & 1 & 0 & 3.517527 & 2.042321 & 1.594192 \\
\hline 22 & 1 & 0 & 3.577092 & 3.356198 & 0.403063 \\
\hline 23 & 6 & 0 & -3.697472 & -2.176589 & -1.867081 \\
\hline 24 & 1 & 0 & -4.758740 & -2.076285 & -2.127052 \\
\hline 25 & 6 & 0 & -3.437408 & -0.172883 & 0.194278 \\
\hline 26 & 6 & 0 & -4.869818 & -0.021189 & -0.005610 \\
\hline 27 & 6 & 0 & -5.048184 & 1.235935 & -0.602765 \\
\hline 28 & 6 & 0 & -2.763257 & -1.066067 & 1.134186 \\
\hline 29 & 6 & 0 & -1.530132 & -0.718807 & 1.728324 \\
\hline 30 & 6 & 0 & -3.318033 & -2.320735 & 1.455409 \\
\hline 31 & 6 & 0 & -0.887002 & -1.596892 & 2.590858 \\
\hline 32 & 1 & 0 & -1.078671 & 0.257512 & 1.525854 \\
\hline 33 & 6 & 0 & -2.661358 & -3.200881 & 2.311346 \\
\hline 34 & 1 & 0 & -4.270119 & -2.628756 & 1.018266 \\
\hline 35 & 6 & 0 & -1.438806 & -2.847532 & 2.877428 \\
\hline 36 & 1 & 0 & 0.057414 & -1.284782 & 3.055320 \\
\hline 37 & 1 & 0 & -3.117240 & -4.170538 & 2.543454 \\
\hline 38 & 1 & 0 & -0.928720 & -3.536055 & 3.561547 \\
\hline 39 & 8 & 0 & -3.871549 & 1.908986 & -0.750406 \\
\hline 40 & 6 & 0 & -2.869371 & 1.159293 & -0.188637 \\
\hline 41 & 8 & 0 & -1.755314 & 1.587478 & -0.100577 \\
\hline 42 & 6 & 0 & -6.281595 & 1.731455 & -0.979843 \\
\hline 43 & 6 & 0 & -6.007866 & -0.806180 & 0.236633 \\
\hline 44 & 6 & 0 & -7.389162 & 0.922685 & -0.734059 \\
\hline 45 & 1 & 0 & -8.386586 & 1.281255 & -1.018194 \\
\hline 46 & 6 & 0 & -7.273247 & -0.338633 & -0.121396 \\
\hline 47 & 1 & 0 & -6.369758 & 2.719108 & -1.442067 \\
\hline 48 & 1 & 0 & -5.934769 & -1.787724 & 0.715547 \\
\hline
\end{tabular}




\begin{tabular}{|c|c|c|c|c|c|}
\hline 49 & 6 & 0 & -8.504484 & -1.150241 & 0.150793 \\
\hline 50 & 1 & 0 & -8.256397 & -2.169592 & 0.494205 \\
\hline 51 & 1 & 0 & -9.130408 & -0.680861 & 0.933708 \\
\hline 52 & 1 & 0 & -9.137467 & -1.241163 & -0.751296 \\
\hline 53 & 1 & 0 & 0.664942 & -3.386932 & -0.789449 \\
\hline 54 & 6 & 0 & 0.747514 & 2.250589 & 1.839400 \\
\hline 55 & 6 & 0 & -0.342863 & 3.106973 & 2.031323 \\
\hline 56 & 6 & 0 & 1.198373 & 1.459871 & 2.908568 \\
\hline 57 & 6 & 0 & -0.961241 & 3.179550 & 3.277877 \\
\hline 58 & 1 & 0 & -0.719280 & 3.711140 & 1.197961 \\
\hline 59 & 6 & 0 & 0.578774 & 1.539559 & 4.152111 \\
\hline 60 & 1 & 0 & 2.040000 & 0.764281 & 2.775560 \\
\hline 61 & 6 & 0 & -0.505193 & 2.397865 & 4.338246 \\
\hline 62 & 1 & 0 & -1.810757 & 3.856716 & 3.421305 \\
\hline 63 & 1 & 0 & 0.944316 & 0.922050 & 4.981260 \\
\hline 64 & 1 & 0 & -0.995852 & 2.458641 & 5.316061 \\
\hline 65 & 6 & 0 & 3.869931 & -0.930208 & 1.340023 \\
\hline 66 & 6 & 0 & 4.989654 & -0.551543 & 2.092282 \\
\hline 67 & 6 & 0 & 2.908708 & -1.768952 & 1.919085 \\
\hline 68 & 6 & 0 & 5.136121 & -0.994190 & 3.405249 \\
\hline 69 & 1 & 0 & 5.760611 & 0.096393 & 1.654747 \\
\hline 70 & 6 & 0 & 3.062412 & -2.220521 & 3.228673 \\
\hline 71 & 1 & 0 & 2.017933 & -2.052885 & 1.340474 \\
\hline 72 & 6 & 0 & 4.172523 & -1.827361 & 3.974598 \\
\hline 73 & 1 & 0 & 6.011284 & -0.687814 & 3.988902 \\
\hline 74 & 1 & 0 & 2.306398 & -2.879716 & 3.671989 \\
\hline 75 & 1 & 0 & 4.291011 & -2.173745 & 5.007376 \\
\hline 76 & 6 & 0 & 4.697828 & -1.232107 & -1.461238 \\
\hline 77 & 6 & 0 & 4.586030 & -0.985456 & -2.837782 \\
\hline 78 & 6 & 0 & 5.635209 & -2.163271 & -0.998895 \\
\hline 79 & 6 & 0 & 5.423270 & -1.638629 & -3.736009 \\
\hline 80 & 1 & 0 & 3.827915 & -0.281356 & -3.209783 \\
\hline 81 & 6 & 0 & 6.464386 & -2.822513 & -1.905179 \\
\hline 82 & 1 & 0 & 5.723034 & -2.377185 & 0.073581 \\
\hline 83 & 6 & 0 & 6.363653 & -2.558401 & -3.269694 \\
\hline 84 & 1 & 0 & 5.335894 & -1.436408 & -4.809260 \\
\hline 85 & 1 & 0 & 7.198730 & -3.548761 & -1.539107 \\
\hline 86 & 1 & 0 & 7.018860 & -3.077887 & -3.977755 \\
\hline 87 & 6 & 0 & 1.002380 & 3.430016 & -0.837977 \\
\hline 88 & 6 & 0 & 1.349028 & 4.746307 & -0.499424 \\
\hline 89 & 6 & 0 & 0.248034 & 3.183955 & -1.990569 \\
\hline 90 & 6 & 0 & 0.957113 & 5.802793 & -1.315311 \\
\hline 91 & 1 & 0 & 1.916521 & 4.955388 & 0.417620 \\
\hline 92 & 6 & 0 & -0.138921 & 4.247135 & -2.805575 \\
\hline 93 & 1 & 0 & -0.053861 & 2.158077 & -2.238438 \\
\hline 94 & 6 & 0 & 0.216921 & 5.551647 & -2.471746 \\
\hline 95 & 1 & 0 & 1.228916 & 6.829764 & -1.046743 \\
\hline 96 & 1 & 0 & -0.728274 & 4.052192 & -3.708289 \\
\hline 97 & 1 & 0 & -0.088511 & 6.384628 & -3.115080 \\
\hline
\end{tabular}

Cartesian coordinates (in $\AA$ ) and energy of TS-Pd-C(6)-B $\mathrm{E}($ UM06) $=-2961.14037618 \mathrm{au}$

\begin{tabular}{cccccc}
$\begin{array}{c}\text { Center } \\
\text { number }\end{array}$ & $\begin{array}{c}\text { Atomic } \\
\text { number }\end{array}$ & $\begin{array}{c}\text { Atomic } \\
\text { type }\end{array}$ & \multicolumn{3}{c}{ Coordinates (Angstroms) } \\
\hline 1 & 46 & 0 & 0.964656 & -0.279605 & -0.644365 \\
2 & 15 & 0 & 3.301081 & -0.345370 & -0.659429 \\
3 & 15 & 0 & 1.251800 & 1.989355 & -0.066970 \\
4 & 8 & 0 & 0.062173 & -2.563902 & -0.179967 \\
5 & 8 & 0 & -1.107506 & -0.557875 & -1.496814 \\
6 & 6 & 0 & -1.789586 & -1.585291 & -1.272687 \\
7 & 6 & 0 & -1.222636 & -2.733484 & -0.588082 \\
8 & 6 & 0 & -3.183509 & -1.697992 & -1.655833 \\
9 & 6 & 0 & -1.947454 & -3.872017 & -0.394324 \\
10 & 6 & 0 & -3.292840 & -4.000031 & -0.873660 \\
11 & 1 & 0 & -1.492132 & -4.722038 & 0.135412 \\
12 & 1 & 0 & -3.544542 & -0.936876 & -2.355237 \\
13 & 6 & 0 & -4.036362 & -5.267841 & -0.612585
\end{tabular}




\begin{tabular}{|c|c|c|c|c|c|}
\hline 14 & 1 & 0 & -5.007855 & -5.284592 & -1.134617 \\
\hline 15 & 1 & 0 & -3.456076 & -6.155984 & -0.927752 \\
\hline 16 & 1 & 0 & -4.238923 & -5.386234 & 0.471163 \\
\hline 17 & 6 & 0 & 3.058578 & 2.400321 & -0.311230 \\
\hline 18 & 6 & 0 & 3.754669 & 1.372971 & -1.191755 \\
\hline 19 & 1 & 0 & 3.420172 & 1.457228 & -2.245263 \\
\hline 20 & 1 & 0 & 4.853013 & 1.512042 & -1.195847 \\
\hline 21 & 1 & 0 & 3.536278 & 2.445570 & 0.685120 \\
\hline 22 & 1 & 0 & 3.131575 & 3.414619 & -0.746182 \\
\hline 23 & 6 & 0 & -3.857720 & -2.940509 & -1.545886 \\
\hline 24 & 1 & 0 & -4.877710 & -3.027947 & -1.938901 \\
\hline 25 & 6 & 0 & -3.980247 & -0.376274 & 0.055362 \\
\hline 26 & 6 & 0 & -3.249678 & -0.867977 & 1.200593 \\
\hline 27 & 6 & 0 & -4.019777 & -1.911740 & 1.736113 \\
\hline 28 & 6 & 0 & -3.943777 & 0.968956 & -0.501793 \\
\hline 29 & 6 & 0 & -4.680650 & 1.299354 & -1.658558 \\
\hline 30 & 6 & 0 & -3.260094 & 2.001485 & 0.169265 \\
\hline 31 & 6 & 0 & -4.723637 & 2.606394 & -2.122411 \\
\hline 32 & 1 & 0 & -5.249692 & 0.522254 & -2.178497 \\
\hline 33 & 6 & 0 & -3.319111 & 3.311363 & -0.292217 \\
\hline 34 & 1 & 0 & -2.731678 & 1.783529 & 1.103499 \\
\hline 35 & 6 & 0 & -4.046368 & 3.619206 & -1.439865 \\
\hline 36 & 1 & 0 & -5.307907 & 2.842719 & -3.018536 \\
\hline 37 & 1 & 0 & -2.803177 & 4.110812 & 0.254582 \\
\hline 38 & 1 & 0 & -4.094941 & 4.653854 & -1.798385 \\
\hline 39 & 8 & 0 & -5.205868 & -2.070059 & 1.081682 \\
\hline 40 & 6 & 0 & -5.275871 & -1.140760 & 0.071116 \\
\hline 41 & 8 & 0 & -6.234987 & -1.037100 & -0.628944 \\
\hline 42 & 6 & 0 & -3.596284 & -2.674375 & 2.809886 \\
\hline 43 & 6 & 0 & -1.992440 & -0.589420 & 1.752703 \\
\hline 44 & 6 & 0 & -2.344180 & -2.372083 & 3.345644 \\
\hline 45 & 1 & 0 & -1.976745 & -2.957071 & 4.198680 \\
\hline 46 & 6 & 0 & -1.532558 & -1.341018 & 2.833519 \\
\hline 47 & 1 & 0 & -4.224783 & -3.474762 & 3.212509 \\
\hline 48 & 1 & 0 & -1.345946 & 0.187957 & 1.322366 \\
\hline 49 & 6 & 0 & -0.197666 & -1.048421 & 3.449608 \\
\hline 50 & 1 & 0 & 0.434074 & -0.438658 & 2.774757 \\
\hline 51 & 1 & 0 & -0.302142 & -0.476049 & 4.392872 \\
\hline 52 & 1 & 0 & 0.349547 & -1.978135 & 3.698307 \\
\hline 53 & 1 & 0 & 0.437578 & -3.392338 & 0.164938 \\
\hline 54 & 6 & 0 & 0.905079 & 2.416449 & 1.670675 \\
\hline 55 & 6 & 0 & -0.314890 & 3.006781 & 2.026484 \\
\hline 56 & 6 & 0 & 1.786622 & 2.012091 & 2.685721 \\
\hline 57 & 6 & 0 & -0.645891 & 3.192069 & 3.367139 \\
\hline 58 & 1 & 0 & -1.006392 & 3.335802 & 1.241134 \\
\hline 59 & 6 & 0 & 1.458468 & 2.211698 & 4.023538 \\
\hline 60 & 1 & 0 & 2.739922 & 1.523224 & 2.442582 \\
\hline 61 & 6 & 0 & 0.240390 & 2.797435 & 4.368829 \\
\hline 62 & 1 & 0 & -1.601026 & 3.660409 & 3.631134 \\
\hline 63 & 1 & 0 & 2.163000 & 1.901047 & 4.804016 \\
\hline 64 & 1 & 0 & -0.017367 & 2.952063 & 5.422460 \\
\hline 65 & 6 & 0 & 4.043334 & -0.531217 & 0.997928 \\
\hline 66 & 6 & 0 & 5.286893 & 0.022473 & 1.332125 \\
\hline 67 & 6 & 0 & 3.310975 & -1.210084 & 1.979437 \\
\hline 68 & 6 & 0 & 5.778671 & -0.089992 & 2.630600 \\
\hline 69 & 1 & 0 & 5.883842 & 0.547593 & 0.574664 \\
\hline 70 & 6 & 0 & 3.807823 & -1.330551 & 3.275958 \\
\hline 71 & 1 & 0 & 2.326963 & -1.629551 & 1.724973 \\
\hline 72 & 6 & 0 & 5.038830 & -0.764314 & 3.603174 \\
\hline 73 & 1 & 0 & 6.749143 & 0.349968 & 2.885745 \\
\hline 74 & 1 & 0 & 3.227497 & -1.863502 & 4.037997 \\
\hline 75 & 1 & 0 & 5.428792 & -0.850665 & 4.623555 \\
\hline 76 & 6 & 0 & 4.222571 & -1.432093 & -1.791389 \\
\hline 77 & 6 & 0 & 5.302218 & -2.222774 & -1.380667 \\
\hline 78 & 6 & 0 & 3.790996 & -1.486815 & -3.125278 \\
\hline 79 & 6 & 0 & 5.954060 & -3.041054 & -2.302112 \\
\hline 80 & 1 & 0 & 5.640558 & -2.202553 & -0.337276 \\
\hline 81 & 6 & 0 & 4.452377 & -2.296815 & -4.042193 \\
\hline 82 & 1 & 0 & 2.920686 & -0.896288 & -3.445912 \\
\hline 83 & 6 & 0 & 5.535190 & -3.075290 & -3.630638 \\
\hline 84 & 1 & 0 & 6.800214 & -3.656676 & -1.977033 \\
\hline 85 & 1 & 0 & 4.115185 & -2.329501 & -5.084120 \\
\hline
\end{tabular}




$\begin{array}{lllrrr}86 & 1 & 0 & 6.051919 & -3.718780 & -4.351297 \\ 87 & 6 & 0 & 0.344893 & 3.172340 & -1.113772 \\ 88 & 6 & 0 & -0.464144 & 2.683976 & -2.146552 \\ 89 & 6 & 0 & 0.497867 & 4.556752 & -0.941260 \\ 90 & 6 & 0 & -1.092454 & 3.576103 & -3.015996 \\ 91 & 1 & 0 & -0.617873 & 1.602594 & -2.263562 \\ 92 & 6 & 0 & -0.141510 & 5.440407 & -1.804781 \\ 93 & 1 & 0 & 1.121232 & 4.948230 & -0.125506 \\ 94 & 6 & 0 & -0.928245 & 4.948622 & -2.849253 \\ 95 & 1 & 0 & -1.720889 & 3.189803 & -3.826504 \\ 96 & 1 & 0 & -0.017672 & 6.520869 & -1.670194 \\ 97 & 1 & 0 & -1.419184 & 5.647454 & -3.536562\end{array}$

Cartesian coordinates (in Å) and energy of TS-Pd-C(6)-C $\mathrm{E}(\mathrm{UM} 06)=-2961.14893398 \mathrm{au}$

\begin{tabular}{|c|c|c|c|c|c|}
\hline \multirow{2}{*}{$\begin{array}{l}\text { Center } \\
\text { number }\end{array}$} & \multirow{2}{*}{$\begin{array}{l}\text { Atomic } \\
\text { number }\end{array}$} & \multirow{2}{*}{$\begin{array}{l}\text { Atomic } \\
\text { type }\end{array}$} & \multicolumn{3}{|c|}{ Coordinates (Angstroms) } \\
\hline & & & $\mathrm{X}$ & $\mathrm{Y}$ & $\mathrm{Z}$ \\
\hline 1 & 46 & 0 & -0.989675 & -0.395358 & 0.213815 \\
\hline 2 & 15 & 0 & -3.185687 & -1.217522 & 0.172085 \\
\hline 3 & 15 & 0 & -1.981896 & 1.703255 & 0.767179 \\
\hline 4 & 8 & 0 & 0.199390 & -2.554541 & -0.016899 \\
\hline 5 & 8 & 0 & 1.254863 & -0.223069 & 0.765963 \\
\hline 6 & 6 & 0 & 1.998689 & -1.228776 & 0.730155 \\
\hline 7 & 6 & 0 & 1.514295 & -2.533879 & 0.313121 \\
\hline 8 & 6 & 0 & 3.418043 & -1.138918 & 1.015807 \\
\hline 9 & 6 & 0 & 2.345248 & -3.610997 & 0.276960 \\
\hline 10 & 6 & 0 & 3.731001 & -3.510604 & 0.642756 \\
\hline 11 & 1 & 0 & 1.958756 & -4.584780 & -0.056364 \\
\hline 12 & 1 & 0 & 3.729578 & -0.218122 & 1.521879 \\
\hline 13 & 6 & 0 & 4.587480 & -4.726152 & 0.527736 \\
\hline 14 & 1 & 0 & 5.593319 & -4.559145 & 0.948987 \\
\hline 15 & 1 & 0 & 4.134734 & -5.597765 & 1.037458 \\
\hline 16 & 1 & 0 & 4.708231 & -5.002236 & -0.538678 \\
\hline 17 & 6 & 0 & -3.797392 & 1.352195 & 0.975760 \\
\hline 18 & 6 & 0 & -4.057486 & -0.095072 & 1.367664 \\
\hline 19 & 1 & 0 & -3.647139 & -0.319365 & 2.373256 \\
\hline 20 & 1 & 0 & -5.140996 & -0.319458 & 1.403164 \\
\hline 21 & 1 & 0 & -4.257350 & 1.571711 & -0.008208 \\
\hline 22 & 1 & 0 & -4.255181 & 2.063460 & 1.688202 \\
\hline 23 & 6 & 0 & 4.223296 & -2.300522 & 1.070842 \\
\hline 24 & 1 & 0 & 5.273852 & -2.210860 & 1.374517 \\
\hline 25 & 6 & 0 & 4.068853 & -0.141619 & -0.909637 \\
\hline 26 & 6 & 0 & 3.201314 & 0.995171 & -0.999765 \\
\hline 27 & 6 & 0 & 2.061975 & 0.568353 & -1.707064 \\
\hline 28 & 6 & 0 & 5.513141 & -0.134673 & -0.662044 \\
\hline 29 & 6 & 0 & 6.374048 & -0.992376 & -1.367840 \\
\hline 30 & 6 & 0 & 6.067777 & 0.724475 & 0.303867 \\
\hline 31 & 6 & 0 & 7.744656 & -0.972095 & -1.125135 \\
\hline 32 & 1 & 0 & 5.965047 & -1.678651 & -2.114304 \\
\hline 33 & 6 & 0 & 7.434528 & 0.733540 & 0.550142 \\
\hline 34 & 1 & 0 & 5.416069 & 1.372161 & 0.901466 \\
\hline 35 & 6 & 0 & 8.280313 & -0.113458 & -0.168238 \\
\hline 36 & 1 & 0 & 8.402049 & -1.639812 & -1.692859 \\
\hline 37 & 1 & 0 & 7.845560 & 1.403721 & 1.313149 \\
\hline 38 & 1 & 0 & 9.358943 & -0.105243 & 0.023726 \\
\hline 39 & 8 & 0 & 2.148756 & -0.727965 & -2.090404 \\
\hline 40 & 6 & 0 & 3.404020 & -1.202813 & -1.723936 \\
\hline 41 & 8 & 0 & 3.770772 & -2.296166 & -2.033591 \\
\hline 42 & 6 & 0 & 1.011825 & 1.418797 & -2.025406 \\
\hline 43 & 6 & 0 & 3.265317 & 2.330706 & -0.559822 \\
\hline 44 & 6 & 0 & 1.111907 & 2.730159 & -1.584338 \\
\hline 45 & 1 & 0 & 0.311406 & 3.437913 & -1.834480 \\
\hline 46 & 6 & 0 & 2.219179 & 3.200313 & -0.838949 \\
\hline 47 & 1 & 0 & 0.159034 & 1.063510 & -2.614624 \\
\hline 48 & 1 & 0 & 4.144799 & 2.706183 & -0.025678 \\
\hline 49 & 6 & 0 & 2.238237 & 4.623468 & -0.369830 \\
\hline 50 & 1 & 0 & 3.226594 & 4.911325 & 0.028944 \\
\hline 51 & 1 & 0 & 1.983572 & 5.323329 & -1.187600 \\
\hline
\end{tabular}




\begin{tabular}{llrrrr}
52 & 1 & 0 & 1.491610 & 4.786759 & 0.433347 \\
53 & 1 & 0 & -0.106611 & -3.444106 & -0.265119 \\
54 & 6 & 0 & -1.996074 & 3.161578 & -0.333735 \\
55 & 6 & 0 & -1.605706 & 4.436536 & 0.090344 \\
56 & 6 & 0 & -2.395400 & 2.969783 & -1.664991 \\
57 & 6 & 0 & -1.619287 & 5.505000 & -0.806936 \\
58 & 1 & 0 & -1.293769 & 4.602685 & 1.129493 \\
59 & 6 & 0 & -2.413627 & 4.039524 & -2.554395 \\
60 & 1 & 0 & -2.691638 & 1.968377 & -2.012469 \\
61 & 6 & 0 & -2.020855 & 5.309791 & -2.126991 \\
62 & 1 & 0 & -1.318332 & 6.502514 & -0.466515 \\
63 & 1 & 0 & -2.732679 & 3.882207 & -3.590850 \\
64 & 1 & 0 & -2.032512 & 6.152012 & -2.827762 \\
65 & 6 & 0 & -4.106417 & -1.019277 & -1.390613 \\
66 & 6 & 0 & -5.507415 & -1.000507 & -1.439262 \\
67 & 6 & 0 & -3.377029 & -0.869284 & -2.576579 \\
68 & 6 & 0 & -6.164011 & -0.831883 & -2.655464 \\
69 & 1 & 0 & -6.097249 & -1.130401 & -0.521913 \\
70 & 6 & 0 & -4.036135 & -0.704321 & -3.794165 \\
71 & 1 & 0 & -2.277970 & -0.882342 & -2.539023 \\
72 & 6 & 0 & -5.428817 & -0.683283 & -3.832505 \\
73 & 1 & 0 & -7.259191 & -0.818411 & -2.686517 \\
74 & 1 & 0 & -3.458226 & -0.592838 & -4.718390 \\
75 & 1 & 0 & -5.948766 & -0.551473 & -4.787995 \\
76 & 6 & 0 & -3.511174 & -2.906599 & 0.772660 \\
77 & 6 & 0 & -2.926308 & -3.284657 & 1.992063 \\
78 & 6 & 0 & -4.247571 & -3.847904 & 0.043042 \\
79 & 6 & 0 & -3.107950 & -4.571353 & 2.488601 \\
80 & 1 & 0 & -2.315318 & -2.565011 & 2.555941 \\
81 & 6 & 0 & -4.416506 & -5.139621 & 0.540263 \\
82 & 1 & 0 & -4.695930 & -3.574986 & -0.920137 \\
83 & 6 & 0 & -3.854498 & -5.501223 & 1.762791 \\
84 & 1 & 0 & -2.658498 & -4.854175 & 3.447157 \\
85 & 1 & 0 & -4.997708 & -5.869397 & -0.034534 \\
86 & 1 & 0 & -3.993704 & -6.516005 & 2.151861 \\
87 & 6 & 0 & -1.338578 & 2.314689 & 2.357393 \\
88 & 6 & 0 & -2.142893 & 2.750933 & 3.415853 \\
89 & 6 & 0 & 0.058556 & 2.374376 & 2.479931 \\
90 & 6 & 0 & -1.558587 & 3.249071 & 4.579632 \\
91 & 1 & 0 & -3.236769 & 2.715181 & 3.345914 \\
92 & 6 & 0 & 0.635454 & 2.888732 & 3.637829 \\
93 & 1 & 0 & 0.696940 & 2.001639 & 1.665492 \\
94 & 6 & 0 & -0.171459 & 3.324537 & 4.689164 \\
95 & 1 & 0 & -2.194926 & 3.586262 & 5.405457 \\
96 & 1 & 0 & 1.726777 & 2.938118 & 3.725678 \\
97 & 1 & 0 & 0.284278 & 3.720736 & 5.603537 \\
\hline------------------------------------------
\end{tabular}

Cartesian coordinates (in $\AA$ ) and energy of TS-Pd-C(6)-D $\mathrm{E}(\mathrm{UM} 06)=-2961.14141153 \mathrm{au}$

\begin{tabular}{cccccc}
$\begin{array}{c}\text { Center } \\
\text { number }\end{array}$ & $\begin{array}{c}\text { Atomic } \\
\text { number }\end{array}$ & $\begin{array}{c}\text { Atomic } \\
\text { type }\end{array}$ & \multicolumn{3}{c}{$\begin{array}{c}\text { Coordinates (Angstroms) } \\
\text { X }\end{array}$} \\
\hline 1 & 46 & 0 & -0.874039 & -0.126421 & -0.283316 \\
3 & 15 & 0 & -3.133463 & -0.568263 & 0.134111 \\
4 & 15 & 0 & -1.281175 & 1.993648 & 0.674199 \\
5 & 8 & 0 & -0.266629 & -2.463354 & -0.814150 \\
6 & 6 & 0 & 1.047307 & -0.271940 & -1.578203 \\
7 & 6 & 0 & 1.643269 & -1.367336 & -1.656077 \\
8 & 6 & 0 & 0.983256 & -2.620777 & -1.312810 \\
9 & 6 & 0 & 3.039940 & -1.485419 & -2.035849 \\
10 & 6 & 0 & 1.602707 & -3.819311 & -1.489188 \\
11 & 1 & 0 & 2.945683 & -3.911754 & -1.995350 \\
12 & 6 & 0 & 1.071658 & -4.747300 & -1.230445 \\
13 & 1 & 0 & 3.569190 & -5.258779 & -2.139862 \\
14 & 1 & 0 & 4.557860 & -5.200009 & -2.624896 \\
15 & 1 & 0 & 3.713281 & -5.730101 & -1.146914 \\
16 & 6 & 0 & 2.929413 & -5.943765 & -2.728639 \\
& 6 & 0 & -3.104534 & 2.027158 & 1.057979
\end{tabular}




\begin{tabular}{|c|c|c|c|c|c|}
\hline 17 & 6 & 0 & -3.635387 & 0.650496 & 1.439141 \\
\hline 18 & 1 & 0 & -3.204127 & 0.290996 & 2.394625 \\
\hline 19 & 1 & 0 & -4.735328 & 0.661492 & 1.562459 \\
\hline 20 & 1 & 0 & -3.594577 & 2.379593 & 0.127320 \\
\hline 21 & 1 & 0 & -3.315725 & 2.786821 & 1.835611 \\
\hline 22 & 6 & 0 & 3.614463 & -2.752529 & -2.304748 \\
\hline 23 & 1 & 0 & 4.638748 & -2.803281 & -2.693472 \\
\hline 24 & 1 & 0 & -0.658184 & -3.300045 & -0.507595 \\
\hline 25 & 6 & 0 & -1.020500 & 3.406825 & -0.444089 \\
\hline 26 & 6 & 0 & -0.729135 & 3.146156 & -1.788705 \\
\hline 27 & 6 & 0 & -1.149470 & 4.730904 & -0.002581 \\
\hline 28 & 6 & 0 & -0.578233 & 4.201927 & -2.687239 \\
\hline 29 & 1 & 0 & -0.605237 & 2.106864 & -2.127586 \\
\hline 30 & 6 & 0 & -0.990010 & 5.781137 & -0.902009 \\
\hline 31 & 1 & 0 & -1.370520 & 4.942694 & 1.052245 \\
\hline 32 & 6 & 0 & -0.707969 & 5.516566 & -2.243849 \\
\hline 33 & 1 & 0 & -0.349373 & 3.995121 & -3.738549 \\
\hline 34 & 1 & 0 & -1.090103 & 6.815838 & -0.555563 \\
\hline 35 & 1 & 0 & -0.585740 & 6.346512 & -2.948920 \\
\hline 36 & 6 & 0 & -4.293885 & -0.245574 & -1.233154 \\
\hline 37 & 6 & 0 & -5.683169 & -0.240367 & -1.037720 \\
\hline 38 & 6 & 0 & -3.774955 & 0.050733 & -2.498879 \\
\hline 39 & 6 & 0 & -6.536440 & 0.052558 & -2.097383 \\
\hline 40 & 1 & 0 & -6.107736 & -0.477156 & -0.052634 \\
\hline 41 & 6 & 0 & -4.632831 & 0.345684 & -3.557980 \\
\hline 42 & 1 & 0 & -2.686080 & 0.050387 & -2.650585 \\
\hline 43 & 6 & 0 & -6.011076 & 0.346553 & -3.356875 \\
\hline 44 & 1 & 0 & -7.620836 & 0.052837 & -1.940448 \\
\hline 45 & 1 & 0 & -4.220529 & 0.576893 & -4.546261 \\
\hline 46 & 1 & 0 & -6.685607 & 0.579126 & -4.188484 \\
\hline 47 & 6 & 0 & -3.492168 & -2.220424 & 0.805473 \\
\hline 48 & 6 & 0 & -2.757331 & -2.629088 & 1.930869 \\
\hline 49 & 6 & 0 & -4.360220 & -3.128762 & 0.187247 \\
\hline 50 & 6 & 0 & -2.914690 & -3.911494 & 2.446435 \\
\hline 51 & 1 & 0 & -2.040151 & -1.938809 & 2.399055 \\
\hline 52 & 6 & 0 & -4.509376 & -4.415389 & 0.704818 \\
\hline 53 & 1 & 0 & -4.925327 & -2.833201 & -0.705250 \\
\hline 54 & 6 & 0 & -3.793583 & -4.807323 & 1.834319 \\
\hline 55 & 1 & 0 & -2.344192 & -4.217727 & 3.331098 \\
\hline 56 & 1 & 0 & -5.195515 & -5.117917 & 0.218662 \\
\hline 57 & 1 & 0 & -3.916953 & -5.818029 & 2.238886 \\
\hline 58 & 6 & 0 & -0.455413 & 2.436333 & 2.238510 \\
\hline 59 & 6 & 0 & -1.027166 & 2.144474 & 3.484715 \\
\hline 60 & 6 & 0 & 0.847101 & 2.955170 & 2.195665 \\
\hline 61 & 6 & 0 & -0.306375 & 2.358388 & 4.658721 \\
\hline 62 & 1 & 0 & -2.048689 & 1.750731 & 3.555865 \\
\hline 63 & 6 & 0 & 1.566254 & 3.162901 & 3.368255 \\
\hline 64 & 1 & 0 & 1.302135 & 3.210170 & 1.230030 \\
\hline 65 & 6 & 0 & 0.993623 & 2.857588 & 4.603426 \\
\hline 66 & 1 & 0 & -0.767744 & 2.133756 & 5.626898 \\
\hline 67 & 1 & 0 & 2.582949 & 3.569238 & 3.316207 \\
\hline 68 & 1 & 0 & 1.560050 & 3.018407 & 5.527144 \\
\hline 69 & 6 & 0 & 3.684672 & -0.862801 & 0.085037 \\
\hline 70 & 6 & 0 & 3.925362 & 0.530219 & -0.242434 \\
\hline 71 & 6 & 0 & 5.275669 & 0.611725 & -0.622919 \\
\hline 72 & 6 & 0 & 5.025526 & -1.529766 & -0.083151 \\
\hline 73 & 8 & 0 & 5.906689 & -0.595490 & -0.562424 \\
\hline 74 & 8 & 0 & 5.356560 & -2.658401 & 0.116990 \\
\hline 75 & 6 & 0 & 2.728845 & -1.387344 & 1.058820 \\
\hline 76 & 6 & 0 & 1.771170 & -0.540729 & 1.651947 \\
\hline 77 & 6 & 0 & 2.749685 & -2.743981 & 1.453939 \\
\hline 78 & 6 & 0 & 0.862452 & -1.027318 & 2.588169 \\
\hline 79 & 1 & 0 & 1.765380 & 0.528304 & 1.425201 \\
\hline 80 & 6 & 0 & 1.832912 & -3.224713 & 2.379875 \\
\hline 81 & 1 & 0 & 3.504904 & -3.413492 & 1.033448 \\
\hline 82 & 6 & 0 & 0.883747 & -2.372881 & 2.950110 \\
\hline 83 & 1 & 0 & 0.151032 & -0.331185 & 3.052854 \\
\hline 84 & 1 & 0 & 1.873810 & -4.278113 & 2.679748 \\
\hline 85 & 1 & 0 & 0.181647 & -2.755662 & 3.700886 \\
\hline 86 & 6 & 0 & 3.131028 & 1.680413 & -0.354492 \\
\hline 87 & 6 & 0 & 5.874123 & 1.782694 & -1.039664 \\
\hline 88 & 6 & 0 & 5.067553 & 2.920781 & -1.093817 \\
\hline
\end{tabular}




$\begin{array}{rrrrrr}89 & 1 & 0 & 5.509904 & 3.870476 & -1.419846 \\ 90 & 6 & 0 & 3.701419 & 2.887817 & -0.768081 \\ 91 & 1 & 0 & 2.051913 & 1.633553 & -0.177799 \\ 92 & 1 & 0 & 6.931573 & 1.802052 & -1.319106 \\ 93 & 6 & 0 & 2.872723 & 4.135883 & -0.853877 \\ 94 & 1 & 0 & 2.915858 & 4.719310 & 0.088122 \\ 95 & 1 & 0 & 3.226686 & 4.805946 & -1.658067 \\ 96 & 1 & 0 & 1.809998 & 3.904399 & -1.052224 \\ 97 & 1 & 0 & 3.492715 & -0.581012 & -2.457014\end{array}$

Cartesian coordinates (in Å) and energy of TS-Pd-C(6)-E $\mathrm{E}(\mathrm{UM} 06)=-2961.14236947 \mathrm{au}$

\begin{tabular}{|c|c|c|c|c|c|}
\hline \multirow{2}{*}{$\begin{array}{l}\text { Center } \\
\text { number }\end{array}$} & \multirow{2}{*}{$\begin{array}{l}\text { Atomic } \\
\text { number }\end{array}$} & \multirow{2}{*}{$\begin{array}{l}\text { Atomic } \\
\text { type }\end{array}$} & \multicolumn{3}{|c|}{ Coordinates (Angstroms) } \\
\hline & & & $\mathrm{X}$ & $\mathrm{Y}$ & $\mathrm{Z}$ \\
\hline 1 & 46 & 0 & -1.220606 & -0.227471 & -0.311496 \\
\hline 2 & 15 & 0 & -3.487827 & -0.647804 & -0.059610 \\
\hline 3 & 15 & 0 & -1.636146 & 1.733340 & 0.909895 \\
\hline 4 & 8 & 0 & 0.017435 & -2.415117 & -0.529126 \\
\hline 5 & 8 & 0 & 0.643208 & -0.034145 & -1.521938 \\
\hline 6 & 6 & 0 & 1.528262 & -0.924257 & -1.547327 \\
\hline 7 & 6 & 0 & 1.253862 & -2.265156 & -1.065137 \\
\hline 8 & 6 & 0 & 2.867313 & -0.676864 & -2.040319 \\
\hline 9 & 6 & 0 & 2.181811 & -3.257929 & -1.183895 \\
\hline 10 & 6 & 0 & 3.463302 & -3.020495 & -1.786903 \\
\hline 11 & 1 & 0 & 1.948155 & -4.268835 & -0.817057 \\
\hline 12 & 6 & 0 & 4.416243 & -4.164479 & -1.916407 \\
\hline 13 & 1 & 0 & 5.374635 & -3.848080 & -2.362497 \\
\hline 14 & 1 & 0 & 4.632122 & -4.622124 & -0.930531 \\
\hline 15 & 1 & 0 & 3.995867 & -4.969465 & -2.550422 \\
\hline 16 & 6 & 0 & -3.470938 & 1.773775 & 1.258291 \\
\hline 17 & 6 & 0 & -4.077198 & 0.379893 & 1.361165 \\
\hline 18 & 1 & 0 & -3.758809 & -0.128264 & 2.292248 \\
\hline 19 & 1 & 0 & -5.184445 & 0.425889 & 1.367444 \\
\hline 20 & 1 & 0 & -3.922819 & 2.323306 & 0.407907 \\
\hline 21 & 1 & 0 & -3.666117 & 2.365758 & 2.172845 \\
\hline 22 & 6 & 0 & 3.761272 & -1.757829 & -2.245188 \\
\hline 23 & 1 & 0 & 4.729954 & -1.562942 & -2.723138 \\
\hline 24 & 1 & 0 & -0.134143 & -3.321476 & -0.210472 \\
\hline 25 & 6 & 0 & -1.272804 & 3.274729 & 0.023617 \\
\hline 26 & 6 & 0 & -0.369878 & 3.245738 & -1.043894 \\
\hline 27 & 6 & 0 & -1.844486 & 4.490293 & 0.428588 \\
\hline 28 & 6 & 0 & -0.039525 & 4.430928 & -1.701162 \\
\hline 29 & 1 & 0 & 0.096725 & 2.300277 & -1.351855 \\
\hline 30 & 6 & 0 & -1.521293 & 5.665057 & -0.241699 \\
\hline 31 & 1 & 0 & -2.536979 & 4.524318 & 1.280305 \\
\hline 32 & 6 & 0 & -0.616961 & 5.634384 & -1.305844 \\
\hline 33 & 1 & 0 & 0.683823 & 4.407501 & -2.523424 \\
\hline 34 & 1 & 0 & -1.970098 & 6.614006 & 0.072487 \\
\hline 35 & 1 & 0 & -0.357572 & 6.563371 & -1.826188 \\
\hline 36 & 6 & 0 & -4.454737 & -0.019015 & -1.476509 \\
\hline 37 & 6 & 0 & -5.819013 & -0.317174 & -1.598505 \\
\hline 38 & 6 & 0 & -3.837067 & 0.806141 & -2.423820 \\
\hline 39 & 6 & 0 & -6.555036 & 0.211906 & -2.654662 \\
\hline 40 & 1 & 0 & -6.310420 & -0.972771 & -0.866479 \\
\hline 41 & 6 & 0 & -4.579831 & 1.337083 & -3.477245 \\
\hline 42 & 1 & 0 & -2.763157 & 1.028576 & -2.337598 \\
\hline 43 & 6 & 0 & -5.936142 & 1.039931 & -3.592347 \\
\hline 44 & 1 & 0 & -7.620665 & -0.025026 & -2.748980 \\
\hline 45 & 1 & 0 & -4.092236 & 1.982500 & -4.216042 \\
\hline 46 & 1 & 0 & -6.518237 & 1.453446 & -4.423557 \\
\hline 47 & 6 & 0 & -4.090807 & -2.338942 & 0.224526 \\
\hline 48 & 6 & 0 & -4.807885 & -2.725180 & 1.362558 \\
\hline 49 & 6 & 0 & -3.784207 & -3.296226 & -0.754891 \\
\hline 50 & 6 & 0 & -5.215560 & -4.050499 & 1.515398 \\
\hline 51 & 1 & 0 & -5.066267 & -1.995899 & 2.139561 \\
\hline 52 & 6 & 0 & -4.198857 & -4.614825 & -0.601399 \\
\hline 53 & 1 & 0 & -3.222516 & -2.997005 & -1.651901 \\
\hline 54 & 6 & 0 & -4.913319 & -4.994147 & 0.536765 \\
\hline 55 & 1 & 0 & -5.781309 & -4.344561 & 2.406476 \\
\hline
\end{tabular}




\begin{tabular}{|c|c|c|c|c|c|}
\hline 56 & 1 & 0 & -3.966828 & -5.354055 & -1.376357 \\
\hline 57 & 1 & 0 & -5.239142 & -6.033121 & 0.658442 \\
\hline 58 & 6 & 0 & -0.832072 & 1.901875 & 2.535881 \\
\hline 59 & 6 & 0 & -1.256888 & 1.111210 & 3.614116 \\
\hline 60 & 6 & 0 & 0.265629 & 2.755591 & 2.701003 \\
\hline 61 & 6 & 0 & -0.610669 & 1.192339 & 4.844461 \\
\hline 62 & 1 & 0 & -2.102553 & 0.420364 & 3.498869 \\
\hline 63 & 6 & 0 & 0.914226 & 2.826878 & 3.931883 \\
\hline 64 & 1 & 0 & 0.621390 & 3.363820 & 1.859903 \\
\hline 65 & 6 & 0 & 0.478458 & 2.050004 & 5.004118 \\
\hline 66 & 1 & 0 & -0.959253 & 0.581664 & 5.685028 \\
\hline 67 & 1 & 0 & 1.769263 & 3.501040 & 4.053893 \\
\hline 68 & 1 & 0 & 0.989366 & 2.112988 & 5.971326 \\
\hline 69 & 6 & 0 & 3.862419 & 0.285240 & -0.238940 \\
\hline 70 & 6 & 0 & 3.553656 & -0.673391 & 0.803573 \\
\hline 71 & 6 & 0 & 2.396521 & -0.197794 & 1.444556 \\
\hline 72 & 6 & 0 & 2.830333 & 1.362833 & -0.087839 \\
\hline 73 & 8 & 0 & 1.984522 & 0.999352 & 0.928508 \\
\hline 74 & 8 & 0 & 2.678463 & 2.383421 & -0.692309 \\
\hline 75 & 6 & 0 & 5.175020 & 0.589930 & -0.799792 \\
\hline 76 & 6 & 0 & 6.329612 & -0.062485 & -0.328350 \\
\hline 77 & 6 & 0 & 5.321949 & 1.543166 & -1.828637 \\
\hline 78 & 6 & 0 & 7.577672 & 0.207142 & -0.878601 \\
\hline 79 & 1 & 0 & 6.258556 & -0.760896 & 0.510384 \\
\hline 80 & 6 & 0 & 6.569837 & 1.804800 & -2.378045 \\
\hline 81 & 1 & 0 & 4.444399 & 2.087611 & -2.190022 \\
\hline 82 & 6 & 0 & 7.702258 & 1.133888 & -1.912279 \\
\hline 83 & 1 & 0 & 8.464242 & -0.304691 & -0.488355 \\
\hline 84 & 1 & 0 & 6.662775 & 2.545920 & -3.179532 \\
\hline 85 & 1 & 0 & 8.685110 & 1.343734 & -2.348658 \\
\hline 86 & 6 & 0 & 4.067403 & -1.917778 & 1.179575 \\
\hline 87 & 6 & 0 & 1.755893 & -0.885362 & 2.450465 \\
\hline 88 & 6 & 0 & 2.300737 & -2.125552 & 2.814520 \\
\hline 89 & 1 & 0 & 1.822666 & -2.698506 & 3.619034 \\
\hline 90 & 6 & 0 & 3.438237 & -2.655739 & 2.194153 \\
\hline 91 & 1 & 0 & 4.950185 & -2.339002 & 0.684248 \\
\hline 92 & 1 & 0 & 0.866234 & -0.470353 & 2.939674 \\
\hline 93 & 6 & 0 & 3.969608 & -4.006258 & 2.574735 \\
\hline 94 & 1 & 0 & 5.071642 & -4.001861 & 2.660890 \\
\hline 95 & 1 & 0 & 3.558791 & -4.351811 & 3.539415 \\
\hline 96 & 1 & 0 & 3.711052 & -4.772362 & 1.816197 \\
\hline 97 & 1 & 0 & 2.996891 & 0.265303 & -2.585929 \\
\hline
\end{tabular}

Cartesian coordinates (in $\AA$ ) and energy of TS-Pd-C(6)-F $\mathrm{E}(\mathrm{UM} 06)=-2961.14586871 \mathrm{au}$

\begin{tabular}{|c|c|c|c|c|c|}
\hline \multirow{2}{*}{$\begin{array}{l}\text { Center } \\
\text { number }\end{array}$} & \multirow{2}{*}{$\begin{array}{l}\text { Atomic } \\
\text { number }\end{array}$} & \multirow{2}{*}{$\begin{array}{l}\text { Atomic } \\
\text { type }\end{array}$} & \multicolumn{3}{|c|}{ Coordinates (Angstroms) } \\
\hline & & & $\mathrm{X}$ & $\mathrm{Y}$ & $\mathrm{Z}$ \\
\hline 1 & 46 & 0 & -1.155532 & -0.272925 & -0.454863 \\
\hline 2 & 15 & 0 & -3.224242 & -1.070600 & 0.281054 \\
\hline 3 & 15 & 0 & -1.962300 & 1.860855 & 0.136158 \\
\hline 4 & 8 & 0 & -0.050623 & -2.425569 & -1.095127 \\
\hline 5 & 8 & 0 & 0.731950 & 0.077383 & -1.657076 \\
\hline 6 & 6 & 0 & 1.591714 & -0.824324 & -1.670143 \\
\hline 7 & 6 & 0 & 1.242074 & -2.229635 & -1.446319 \\
\hline 8 & 6 & 0 & 3.004811 & -0.554204 & -1.847641 \\
\hline 9 & 6 & 0 & 2.162243 & -3.217397 & -1.608003 \\
\hline 10 & 6 & 0 & 3.526345 & -2.926186 & -1.966466 \\
\hline 11 & 1 & 0 & 1.868312 & -4.265583 & -1.453093 \\
\hline 12 & 6 & 0 & 4.472522 & -4.063767 & -2.157868 \\
\hline 13 & 1 & 0 & 5.495286 & -3.710338 & -2.374105 \\
\hline 14 & 1 & 0 & 4.514685 & -4.699440 & -1.251554 \\
\hline 15 & 1 & 0 & 4.150689 & -4.720215 & -2.989911 \\
\hline 16 & 6 & 0 & -3.791928 & 1.632528 & 0.372652 \\
\hline 17 & 6 & 0 & -4.030520 & 0.348175 & 1.158270 \\
\hline 18 & 1 & 0 & -3.551168 & 0.400685 & 2.157018 \\
\hline 19 & 1 & 0 & -5.106516 & 0.163282 & 1.337551 \\
\hline 20 & 1 & 0 & -4.258798 & 1.576414 & -0.632400 \\
\hline 21 & 1 & 0 & -4.220535 & 2.507149 & 0.900039 \\
\hline
\end{tabular}




\begin{tabular}{|c|c|c|c|c|c|}
\hline 22 & 6 & 0 & 3.907565 & -1.617122 & -2.117821 \\
\hline 23 & 1 & 0 & 4.946248 & -1.382543 & -2.382911 \\
\hline 24 & 1 & 0 & -0.220485 & -3.343320 & -0.817879 \\
\hline 25 & 6 & 0 & -1.746372 & 3.233540 & -1.033951 \\
\hline 26 & 6 & 0 & -0.510397 & 3.325283 & -1.690386 \\
\hline 27 & 6 & 0 & -2.748667 & 4.174762 & -1.305610 \\
\hline 28 & 6 & 0 & -0.277822 & 4.362386 & -2.590263 \\
\hline 29 & 1 & 0 & 0.268186 & 2.570385 & -1.507282 \\
\hline 30 & 6 & 0 & -2.511037 & 5.204661 & -2.213057 \\
\hline 31 & 1 & 0 & -3.725213 & 4.111938 & -0.808754 \\
\hline 32 & 6 & 0 & -1.275329 & 5.300735 & -2.852110 \\
\hline 33 & 1 & 0 & 0.689437 & 4.429349 & -3.101496 \\
\hline 34 & 1 & 0 & -3.298079 & 5.937496 & -2.423265 \\
\hline 35 & 1 & 0 & -1.092496 & 6.110569 & -3.567399 \\
\hline 36 & 6 & 0 & -4.378933 & -1.558949 & -1.045883 \\
\hline 37 & 6 & 0 & -5.762901 & -1.350958 & -0.982606 \\
\hline 38 & 6 & 0 & -3.831823 & -2.180572 & -2.176685 \\
\hline 39 & 6 & 0 & -6.583838 & -1.767667 & -2.028662 \\
\hline 40 & 1 & 0 & -6.219246 & -0.863515 & -0.111795 \\
\hline 41 & 6 & 0 & -4.655094 & -2.602751 & -3.217087 \\
\hline 42 & 1 & 0 & -2.743867 & -2.321849 & -2.244167 \\
\hline 43 & 6 & 0 & -6.031856 & -2.395695 & -3.144019 \\
\hline 44 & 1 & 0 & -7.665123 & -1.600060 & -1.970819 \\
\hline 45 & 1 & 0 & -4.217618 & -3.087068 & -4.097110 \\
\hline 46 & 1 & 0 & -6.679861 & -2.720559 & -3.965640 \\
\hline 47 & 6 & 0 & -3.185771 & -2.446288 & 1.475766 \\
\hline 48 & 6 & 0 & -1.992663 & -2.672427 & 2.176956 \\
\hline 49 & 6 & 0 & -4.297737 & -3.263437 & 1.713702 \\
\hline 50 & 6 & 0 & -1.922523 & -3.696244 & 3.120254 \\
\hline 51 & 1 & 0 & -1.108291 & -2.048988 & 1.974050 \\
\hline 52 & 6 & 0 & -4.219925 & -4.287066 & 2.654734 \\
\hline 53 & 1 & 0 & -5.230113 & -3.105989 & 1.155800 \\
\hline 54 & 6 & 0 & -3.035048 & -4.501765 & 3.359240 \\
\hline 55 & 1 & 0 & -0.989539 & -3.868752 & 3.668492 \\
\hline 56 & 1 & 0 & -5.091656 & -4.924948 & 2.838789 \\
\hline 57 & 1 & 0 & -2.977727 & -5.309307 & 4.097805 \\
\hline 58 & 6 & 0 & -1.448982 & 2.492607 & 1.776741 \\
\hline 59 & 6 & 0 & -0.857817 & 1.618509 & 2.696423 \\
\hline 60 & 6 & 0 & -1.679634 & 3.826457 & 2.138829 \\
\hline 61 & 6 & 0 & -0.500884 & 2.074910 & 3.965190 \\
\hline 62 & 1 & 0 & -0.651509 & 0.578275 & 2.405374 \\
\hline 63 & 6 & 0 & -1.321137 & 4.278266 & 3.406370 \\
\hline 64 & 1 & 0 & -2.132787 & 4.523530 & 1.420896 \\
\hline 65 & 6 & 0 & -0.729921 & 3.403273 & 4.319089 \\
\hline 66 & 1 & 0 & -0.033690 & 1.387789 & 4.679523 \\
\hline 67 & 1 & 0 & -1.501576 & 5.322901 & 3.684083 \\
\hline 68 & 1 & 0 & -0.444545 & 3.762070 & 5.314370 \\
\hline 69 & 6 & 0 & 3.406361 & 0.154962 & 0.241879 \\
\hline 70 & 6 & 0 & 4.732533 & -0.405745 & 0.407007 \\
\hline 71 & 6 & 0 & 4.550504 & -1.683583 & 0.949588 \\
\hline 72 & 6 & 0 & 2.506539 & -0.810791 & 0.955054 \\
\hline 73 & 8 & 0 & 3.237784 & -1.931569 & 1.248669 \\
\hline 74 & 8 & 0 & 1.347285 & -0.726184 & 1.264845 \\
\hline 75 & 6 & 0 & 3.085290 & 1.580142 & 0.141853 \\
\hline 76 & 6 & 0 & 2.061415 & 2.151607 & 0.915853 \\
\hline 77 & 6 & 0 & 3.837955 & 2.428694 & -0.693826 \\
\hline 78 & 6 & 0 & 1.819599 & 3.522182 & 0.876858 \\
\hline 79 & 1 & 0 & 1.461301 & 1.517920 & 1.573758 \\
\hline 80 & 6 & 0 & 3.582942 & 3.792431 & -0.744588 \\
\hline 81 & 1 & 0 & 4.625455 & 2.010527 & -1.332487 \\
\hline 82 & 6 & 0 & 2.575544 & 4.347433 & 0.048638 \\
\hline 83 & 1 & 0 & 1.024317 & 3.943396 & 1.504684 \\
\hline 84 & 1 & 0 & 4.177772 & 4.431759 & -1.406428 \\
\hline 85 & 1 & 0 & 2.379282 & 5.424992 & 0.014281 \\
\hline 86 & 6 & 0 & 6.028386 & 0.019941 & 0.093720 \\
\hline 87 & 6 & 0 & 5.592680 & -2.564302 & 1.168416 \\
\hline 88 & 6 & 0 & 6.870478 & -2.124709 & 0.824121 \\
\hline 89 & 1 & 0 & 7.725076 & -2.794925 & 0.981396 \\
\hline 90 & 6 & 0 & 7.109642 & -0.838856 & 0.301903 \\
\hline 91 & 1 & 0 & 6.210808 & 1.025889 & -0.302030 \\
\hline 92 & 1 & 0 & 5.413870 & -3.554548 & 1.598925 \\
\hline 93 & 6 & 0 & 8.513198 & -0.400536 & 0.008125 \\
\hline
\end{tabular}




$\begin{array}{cccccc}94 & 1 & 0 & 9.065594 & -0.173026 & 0.940231 \\ 95 & 1 & 0 & 9.083511 & -1.188448 & -0.516953 \\ 96 & 1 & 0 & 8.535960 & 0.509325 & -0.616515 \\ 97 & 1 & 0 & 3.235408 & 0.454960 & -2.203493\end{array}$

Cartesian coordinates (in ^̊) and energy of INT-II-Pd-C(6)-A $\mathrm{E}(\mathrm{UM} 06)=-2961.16322856 \mathrm{au}$

\begin{tabular}{|c|c|c|c|c|c|}
\hline \multirow{2}{*}{$\begin{array}{l}\text { Center } \\
\text { number }\end{array}$} & \multirow{2}{*}{$\begin{array}{l}\text { Atomic } \\
\text { number }\end{array}$} & \multirow{2}{*}{$\begin{array}{l}\text { Atomic } \\
\text { type }\end{array}$} & \multicolumn{3}{|c|}{ Coordinates (Angstroms) } \\
\hline & & & $\mathrm{X}$ & $\mathrm{Y}$ & $\mathrm{Z}$ \\
\hline 1 & 46 & 0 & 1.371434 & -0.205252 & -0.823193 \\
\hline 2 & 15 & 0 & 3.635313 & -0.430353 & -0.294433 \\
\hline 3 & 15 & 0 & 1.610571 & 2.028495 & -0.048747 \\
\hline 4 & 8 & 0 & 0.332161 & -2.560314 & -0.807511 \\
\hline 5 & 8 & 0 & -0.715975 & -0.344054 & -1.827214 \\
\hline 6 & 6 & 0 & -1.492275 & -1.239213 & -1.515259 \\
\hline 7 & 6 & 0 & -0.995120 & -2.547309 & -1.069652 \\
\hline 8 & 6 & 0 & -2.992139 & -1.046439 & -1.554084 \\
\hline 9 & 6 & 0 & -1.818203 & -3.619006 & -1.037555 \\
\hline 10 & 6 & 0 & -3.223113 & -3.526866 & -1.415452 \\
\hline 11 & 1 & 0 & -1.415181 & -4.605868 & -0.765420 \\
\hline 12 & 1 & 0 & -3.211786 & -0.373044 & -2.406621 \\
\hline 13 & 6 & 0 & -4.004449 & -4.799186 & -1.485510 \\
\hline 14 & 1 & 0 & -5.048461 & -4.619594 & -1.795214 \\
\hline 15 & 1 & 0 & -3.553925 & -5.513162 & -2.201697 \\
\hline 16 & 1 & 0 & -4.020622 & -5.305650 & -0.500295 \\
\hline 17 & 6 & 0 & 3.436077 & 2.289032 & 0.252629 \\
\hline 18 & 6 & 0 & 4.291316 & 1.290369 & -0.513046 \\
\hline 19 & 1 & 0 & 4.255981 & 1.487229 & -1.603503 \\
\hline 20 & 1 & 0 & 5.356156 & 1.349055 & -0.215248 \\
\hline 21 & 1 & 0 & 3.612722 & 2.196710 & 1.340542 \\
\hline 22 & 1 & 0 & 3.700072 & 3.326630 & -0.023703 \\
\hline 23 & 6 & 0 & -3.755650 & -2.320829 & -1.694250 \\
\hline 24 & 1 & 0 & -4.800094 & -2.245782 & -2.016501 \\
\hline 25 & 6 & 0 & -3.464267 & -0.254033 & -0.234320 \\
\hline 26 & 6 & 0 & -4.975849 & -0.125384 & -0.180549 \\
\hline 27 & 6 & 0 & -5.286106 & 1.223241 & -0.311567 \\
\hline 28 & 6 & 0 & -2.807352 & -0.871978 & 1.006077 \\
\hline 29 & 6 & 0 & -1.533598 & -0.469524 & 1.438221 \\
\hline 30 & 6 & 0 & -3.427595 & -1.926041 & 1.687540 \\
\hline 31 & 6 & 0 & -0.902141 & -1.115172 & 2.499995 \\
\hline 32 & 1 & 0 & -1.013561 & 0.361709 & 0.948168 \\
\hline 33 & 6 & 0 & -2.790076 & -2.579371 & 2.740411 \\
\hline 34 & 1 & 0 & -4.430141 & -2.253755 & 1.403275 \\
\hline 35 & 6 & 0 & -1.519435 & -2.181473 & 3.148867 \\
\hline 36 & 1 & 0 & 0.085148 & -0.759065 & 2.822152 \\
\hline 37 & 1 & 0 & -3.302718 & -3.400933 & 3.253578 \\
\hline 38 & 1 & 0 & -1.023639 & -2.688342 & 3.985098 \\
\hline 39 & 8 & 0 & -4.171615 & 2.010810 & -0.449966 \\
\hline 40 & 6 & 0 & -3.062207 & 1.235775 & -0.402150 \\
\hline 41 & 8 & 0 & -1.973594 & 1.714386 & -0.496324 \\
\hline 42 & 6 & 0 & -6.575846 & 1.722142 & -0.309612 \\
\hline 43 & 6 & 0 & -6.035304 & -1.025872 & -0.049256 \\
\hline 44 & 6 & 0 & -7.610320 & 0.801552 & -0.167619 \\
\hline 45 & 1 & 0 & -8.648562 & 1.156115 & -0.164392 \\
\hline 46 & 6 & 0 & -7.360559 & -0.571638 & -0.034756 \\
\hline 47 & 1 & 0 & -6.757361 & 2.795386 & -0.419807 \\
\hline 48 & 1 & 0 & -5.861261 & -2.105167 & 0.031730 \\
\hline 49 & 6 & 0 & -8.498621 & -1.532940 & 0.139458 \\
\hline 50 & 1 & 0 & -8.196659 & -2.571803 & -0.082226 \\
\hline 51 & 1 & 0 & -8.882524 & -1.518243 & 1.178039 \\
\hline 52 & 1 & 0 & -9.348991 & -1.279181 & -0.519053 \\
\hline 53 & 1 & 0 & 0.648733 & -3.455349 & -0.594547 \\
\hline 54 & 6 & 0 & 0.825768 & 2.409847 & 1.551974 \\
\hline 55 & 6 & 0 & -0.334470 & 3.192120 & 1.617253 \\
\hline 56 & 6 & 0 & 1.317753 & 1.809442 & 2.722187 \\
\hline 57 & 6 & 0 & -0.980029 & 3.379964 & 2.837817 \\
\hline 58 & 1 & 0 & -0.746240 & 3.643338 & 0.707036 \\
\hline 59 & 6 & 0 & 0.671301 & 2.006475 & 3.938512 \\
\hline
\end{tabular}




\begin{tabular}{|c|c|c|c|c|c|}
\hline 60 & 1 & 0 & 2.212212 & 1.170838 & 2.692924 \\
\hline 61 & 6 & 0 & -0.480726 & 2.790988 & 3.998394 \\
\hline 62 & 1 & 0 & -1.884855 & 3.996621 & 2.880411 \\
\hline 63 & 1 & 0 & 1.070408 & 1.538913 & 4.846496 \\
\hline 64 & 1 & 0 & -0.991791 & 2.943892 & 4.955475 \\
\hline 65 & 6 & 0 & 3.877017 & -0.801583 & 1.477948 \\
\hline 66 & 6 & 0 & 4.990164 & -0.347436 & 2.197934 \\
\hline 67 & 6 & 0 & 2.878819 & -1.522499 & 2.145614 \\
\hline 68 & 6 & 0 & 5.092624 & -0.597613 & 3.564610 \\
\hline 69 & 1 & 0 & 5.790351 & 0.209169 & 1.692253 \\
\hline 70 & 6 & 0 & 2.987419 & -1.781506 & 3.510988 \\
\hline 71 & 1 & 0 & 1.993864 & -1.864946 & 1.589437 \\
\hline 72 & 6 & 0 & 4.090683 & -1.312384 & 4.222327 \\
\hline 73 & 1 & 0 & 5.962908 & -0.232890 & 4.121448 \\
\hline 74 & 1 & 0 & 2.200447 & -2.347117 & 4.024128 \\
\hline 75 & 1 & 0 & 4.174056 & -1.506601 & 5.297513 \\
\hline 76 & 6 & 0 & 4.798262 & -1.477491 & -1.225651 \\
\hline 77 & 6 & 0 & 4.759667 & -1.398580 & -2.625850 \\
\hline 78 & 6 & 0 & 5.690469 & -2.361857 & -0.608143 \\
\hline 79 & 6 & 0 & 5.624859 & -2.169659 & -3.395056 \\
\hline 80 & 1 & 0 & 4.037399 & -0.731909 & -3.118615 \\
\hline 81 & 6 & 0 & 6.547763 & -3.140099 & -1.384735 \\
\hline 82 & 1 & 0 & 5.721278 & -2.446071 & 0.485260 \\
\hline 83 & 6 & 0 & 6.520414 & -3.041724 & -2.774362 \\
\hline 84 & 1 & 0 & 5.595024 & -2.097006 & -4.487916 \\
\hline 85 & 1 & 0 & 7.246498 & -3.828723 & -0.896522 \\
\hline 86 & 1 & 0 & 7.197783 & -3.653641 & -3.380367 \\
\hline 87 & 6 & 0 & 1.105157 & 3.341280 & -1.203413 \\
\hline 88 & 6 & 0 & 1.467141 & 4.678721 & -0.986620 \\
\hline 89 & 6 & 0 & 0.325057 & 3.003398 & -2.314205 \\
\hline 90 & 6 & 0 & 1.067311 & 5.663089 & -1.884354 \\
\hline 91 & 1 & 0 & 2.052815 & 4.960696 & -0.101078 \\
\hline 92 & 6 & 0 & -0.072113 & 3.994337 & -3.211308 \\
\hline 93 & 1 & 0 & 0.011103 & 1.962118 & -2.463300 \\
\hline 94 & 6 & 0 & 0.301399 & 5.319187 & -2.999785 \\
\hline 95 & 1 & 0 & 1.351479 & 6.707202 & -1.711975 \\
\hline 96 & 1 & 0 & -0.682310 & 3.727556 & -4.081213 \\
\hline 97 & 1 & 0 & -0.011087 & 6.095952 & -3.706878 \\
\hline
\end{tabular}

Cartesian coordinates (in Å) and energy of INT-II-Pd-C(6)-B $\mathrm{E}(\mathrm{UM} 06)=-2961.16829199 \mathrm{au}$

\begin{tabular}{|c|c|c|c|c|c|}
\hline \multirow{2}{*}{$\begin{array}{l}\text { Center } \\
\text { number }\end{array}$} & \multirow{2}{*}{$\begin{array}{l}\text { Atomic } \\
\text { number }\end{array}$} & \multirow{2}{*}{$\begin{array}{l}\text { Atomic } \\
\text { type }\end{array}$} & \multicolumn{3}{|c|}{ Coordinates (Angstroms) } \\
\hline & & & $\mathrm{X}$ & $\mathrm{Y}$ & $\mathrm{Z}$ \\
\hline 1 & 46 & 0 & 1.075921 & -0.337773 & -0.675444 \\
\hline 2 & 15 & 0 & 3.413420 & -0.298466 & -0.518222 \\
\hline 3 & 15 & 0 & 1.200792 & 1.921884 & 0.022592 \\
\hline 4 & 8 & 0 & 0.294770 & -2.718764 & -0.518848 \\
\hline 5 & 8 & 0 & -1.078433 & -0.674178 & -1.497718 \\
\hline 6 & 6 & 0 & -1.703593 & -1.707035 & -1.261025 \\
\hline 7 & 6 & 0 & -1.028856 & -2.901187 & -0.756794 \\
\hline 8 & 6 & 0 & -3.201425 & -1.763750 & -1.424339 \\
\hline 9 & 6 & 0 & -1.701113 & -4.061695 & -0.572212 \\
\hline 10 & 6 & 0 & -3.120386 & -4.200081 & -0.851616 \\
\hline 11 & 1 & 0 & -1.156799 & -4.945792 & -0.207422 \\
\hline 12 & 1 & 0 & -3.424549 & -1.408649 & -2.450988 \\
\hline 13 & 6 & 0 & -3.734958 & -5.552435 & -0.683358 \\
\hline 14 & 1 & 0 & -4.811945 & -5.539513 & -0.920433 \\
\hline 15 & 1 & 0 & -3.248537 & -6.299123 & -1.340063 \\
\hline 16 & 1 & 0 & -3.618881 & -5.915044 & 0.356092 \\
\hline 17 & 6 & 0 & 3.005150 & 2.405416 & -0.038181 \\
\hline 18 & 6 & 0 & 3.814485 & 1.468088 & -0.921431 \\
\hline 19 & 1 & 0 & 3.556212 & 1.603615 & -1.991010 \\
\hline 20 & 1 & 0 & 4.901691 & 1.656609 & -0.829807 \\
\hline 21 & 1 & 0 & 3.397955 & 2.395669 & 0.995267 \\
\hline 22 & 1 & 0 & 3.074095 & 3.449927 & -0.395622 \\
\hline 23 & 6 & 0 & -3.813201 & -3.117761 & -1.258135 \\
\hline 24 & 1 & 0 & -4.878814 & -3.214955 & -1.500514 \\
\hline 25 & 6 & 0 & -3.833775 & -0.694670 & -0.440910 \\
\hline
\end{tabular}




\begin{tabular}{|c|c|c|c|c|c|}
\hline 26 & 6 & 0 & -3.365835 & -0.931331 & 0.971219 \\
\hline 27 & 6 & 0 & -4.446334 & -1.413236 & 1.697893 \\
\hline 28 & 6 & 0 & -3.740276 & 0.739438 & -0.944048 \\
\hline 29 & 6 & 0 & -4.161678 & 1.058832 & -2.241971 \\
\hline 30 & 6 & 0 & -3.373919 & 1.782930 & -0.088550 \\
\hline 31 & 6 & 0 & -4.216322 & 2.381105 & -2.669240 \\
\hline 32 & 1 & 0 & -4.499449 & 0.271153 & -2.925316 \\
\hline 33 & 6 & 0 & -3.427691 & 3.109654 & -0.518128 \\
\hline 34 & 1 & 0 & -3.070724 & 1.562969 & 0.942139 \\
\hline 35 & 6 & 0 & -3.850419 & 3.414547 & -1.807195 \\
\hline 36 & 1 & 0 & -4.565784 & 2.606300 & -3.683386 \\
\hline 37 & 1 & 0 & -3.153046 & 3.918527 & 0.171010 \\
\hline 38 & 1 & 0 & -3.904410 & 4.457818 & -2.138715 \\
\hline 39 & 8 & 0 & -5.598145 & -1.475539 & 0.952800 \\
\hline 40 & 6 & 0 & -5.337322 & -1.048070 & -0.313384 \\
\hline 41 & 8 & 0 & -6.165347 & -1.003311 & -1.165387 \\
\hline 42 & 6 & 0 & -4.349309 & -1.785602 & 3.027743 \\
\hline 43 & 6 & 0 & -2.132641 & -0.784260 & 1.598968 \\
\hline 44 & 6 & 0 & -3.099948 & -1.651758 & 3.633249 \\
\hline 45 & 1 & 0 & -2.984638 & -1.941632 & 4.685449 \\
\hline 46 & 6 & 0 & -1.986379 & -1.148731 & 2.943089 \\
\hline 47 & 1 & 0 & -5.220674 & -2.167325 & 3.568222 \\
\hline 48 & 1 & 0 & -1.270668 & -0.360110 & 1.061367 \\
\hline 49 & 6 & 0 & -0.667900 & -0.975901 & 3.635627 \\
\hline 50 & 1 & 0 & 0.156115 & -0.850220 & 2.906658 \\
\hline 51 & 1 & 0 & -0.662837 & -0.072041 & 4.276732 \\
\hline 52 & 1 & 0 & -0.430407 & -1.838102 & 4.286842 \\
\hline 53 & 1 & 0 & 0.733465 & -3.546117 & -0.255956 \\
\hline 54 & 6 & 0 & 0.674914 & 2.320086 & 1.721658 \\
\hline 55 & 6 & 0 & -0.599242 & 2.855069 & 1.952186 \\
\hline 56 & 6 & 0 & 1.482239 & 1.983333 & 2.819232 \\
\hline 57 & 6 & 0 & -1.049716 & 3.069196 & 3.252800 \\
\hline 58 & 1 & 0 & -1.237766 & 3.117955 & 1.100183 \\
\hline 59 & 6 & 0 & 1.034120 & 2.212841 & 4.117059 \\
\hline 60 & 1 & 0 & 2.473673 & 1.533663 & 2.673475 \\
\hline 61 & 6 & 0 & -0.231513 & 2.756591 & 4.338031 \\
\hline 62 & 1 & 0 & -2.045279 & 3.496987 & 3.418970 \\
\hline 63 & 1 & 0 & 1.682286 & 1.960499 & 4.964494 \\
\hline 64 & 1 & 0 & -0.581340 & 2.938615 & 5.360434 \\
\hline 65 & 6 & 0 & 3.983334 & -0.511362 & 1.203531 \\
\hline 66 & 6 & 0 & 5.182402 & 0.044916 & 1.670223 \\
\hline 67 & 6 & 0 & 3.158903 & -1.206974 & 2.096406 \\
\hline 68 & 6 & 0 & 5.540468 & -0.083818 & 3.010087 \\
\hline 69 & 1 & 0 & 5.848749 & 0.584335 & 0.983752 \\
\hline 70 & 6 & 0 & 3.521738 & -1.342864 & 3.435647 \\
\hline 71 & 1 & 0 & 2.211115 & -1.632272 & 1.734543 \\
\hline 72 & 6 & 0 & 4.709689 & -0.775660 & 3.893298 \\
\hline 73 & 1 & 0 & 6.477023 & 0.357473 & 3.368727 \\
\hline 74 & 1 & 0 & 2.869638 & -1.889081 & 4.126945 \\
\hline 75 & 1 & 0 & 4.994867 & -0.874751 & 4.946672 \\
\hline 76 & 6 & 0 & 4.520921 & -1.272858 & -1.584378 \\
\hline 77 & 6 & 0 & 5.562412 & -2.062278 & -1.082458 \\
\hline 78 & 6 & 0 & 4.275686 & -1.247630 & -2.965766 \\
\hline 79 & 6 & 0 & 6.362067 & -2.796705 & -1.956994 \\
\hline 80 & 1 & 0 & 5.756025 & -2.105717 & -0.003529 \\
\hline 81 & 6 & 0 & 5.083391 & -1.974363 & -3.834031 \\
\hline 82 & 1 & 0 & 3.437107 & -0.659508 & -3.365400 \\
\hline 83 & 6 & 0 & 6.128557 & -2.749771 & -3.329809 \\
\hline 84 & 1 & 0 & 7.178467 & -3.409981 & -1.559409 \\
\hline 85 & 1 & 0 & 4.891667 & -1.943101 & -4.912320 \\
\hline 86 & 1 & 0 & 6.761380 & -3.326592 & -4.013337 \\
\hline 87 & 6 & 0 & 0.369560 & 3.099738 & -1.090762 \\
\hline 88 & 6 & 0 & -0.280348 & 2.611681 & -2.229548 \\
\hline 89 & 6 & 0 & 0.435431 & 4.483092 & -0.864900 \\
\hline 90 & 6 & 0 & -0.831934 & 3.501730 & -3.151038 \\
\hline 91 & 1 & 0 & -0.364587 & 1.528907 & -2.392326 \\
\hline 92 & 6 & 0 & -0.129968 & 5.365010 & -1.780121 \\
\hline 93 & 1 & 0 & 0.932263 & 4.875085 & 0.033161 \\
\hline 94 & 6 & 0 & -0.752620 & 4.873824 & -2.929836 \\
\hline 95 & 1 & 0 & -1.332553 & 3.114974 & -4.045709 \\
\hline 96 & 1 & 0 & -0.075459 & 6.444923 & -1.602289 \\
\hline 97 & 1 & 0 & -1.184062 & 5.572267 & -3.65622 \\
\hline
\end{tabular}


Cartesian coordinates (in $\AA$ ) and energy of INT-II-Pd-C(6)-C $\mathrm{E}(\mathrm{UM} 06)=-2961.16986503 \mathrm{au}$

\begin{tabular}{|c|c|c|c|c|c|}
\hline \multirow{2}{*}{$\begin{array}{l}\text { Center } \\
\text { number }\end{array}$} & \multirow{2}{*}{$\begin{array}{l}\text { Atomic } \\
\text { number }\end{array}$} & \multirow{2}{*}{$\begin{array}{l}\text { Atomic } \\
\text { type }\end{array}$} & \multicolumn{3}{|c|}{ Coordinates (Angstroms) } \\
\hline & & & $\mathrm{X}$ & $\mathrm{Y}$ & $\mathrm{Z}$ \\
\hline 1 & 46 & 0 & 1.842564 & 0.040725 & -0.899469 \\
\hline 2 & 15 & 0 & 2.981526 & -1.525597 & 0.460326 \\
\hline 3 & 15 & 0 & 2.882230 & 1.704816 & 0.375249 \\
\hline 4 & 8 & 0 & 0.544678 & -1.569567 & -1.899809 \\
\hline 5 & 8 & 0 & -0.167940 & 0.953817 & -2.281508 \\
\hline 6 & 6 & 0 & -1.157632 & 0.057814 & -2.064862 \\
\hline 7 & 6 & 0 & -0.665780 & -1.326404 & -1.900219 \\
\hline 8 & 6 & 0 & -2.469909 & 0.334425 & -2.017963 \\
\hline 9 & 6 & 0 & -1.670092 & -2.357882 & -1.774146 \\
\hline 10 & 6 & 0 & -2.993369 & -2.103905 & -1.646821 \\
\hline 11 & 1 & 0 & -1.290092 & -3.386356 & -1.756486 \\
\hline 12 & 1 & 0 & -0.516936 & 1.859944 & -2.345922 \\
\hline 13 & 1 & 0 & -2.813048 & 1.366648 & -2.175398 \\
\hline 14 & 6 & 0 & -3.507511 & -0.685931 & -1.671262 \\
\hline 15 & 1 & 0 & -4.308268 & -0.640614 & -2.441865 \\
\hline 16 & 6 & 0 & -3.950656 & -3.239970 & -1.508812 \\
\hline 17 & 1 & 0 & -4.833840 & -3.117771 & -2.162162 \\
\hline 18 & 1 & 0 & -4.337546 & -3.318068 & -0.474174 \\
\hline 19 & 1 & 0 & -3.456365 & -4.194666 & -1.756759 \\
\hline 20 & 6 & 0 & -4.216540 & -0.226961 & -0.326864 \\
\hline 21 & 6 & 0 & -4.586359 & 1.238988 & -0.358690 \\
\hline 22 & 6 & 0 & -3.793941 & 1.894751 & 0.573951 \\
\hline 23 & 6 & 0 & -5.422643 & -1.067921 & 0.065317 \\
\hline 24 & 6 & 0 & -6.469595 & -1.237488 & -0.849299 \\
\hline 25 & 6 & 0 & -5.549802 & -1.633979 & 1.337139 \\
\hline 26 & 6 & 0 & -7.598574 & -1.977778 & -0.514490 \\
\hline 27 & 1 & 0 & -6.405177 & -0.801313 & -1.854698 \\
\hline 28 & 6 & 0 & -6.683451 & -2.373169 & 1.675199 \\
\hline 29 & 1 & 0 & -4.753826 & -1.520552 & 2.081339 \\
\hline 30 & 6 & 0 & -7.707439 & -2.554335 & 0.750707 \\
\hline 31 & 1 & 0 & -8.401231 & -2.106104 & -1.249184 \\
\hline 32 & 1 & 0 & -6.760898 & -2.813543 & 2.675429 \\
\hline 33 & 1 & 0 & -8.594234 & -3.140317 & 1.015566 \\
\hline 34 & 6 & 0 & -3.118616 & -0.226491 & 0.749939 \\
\hline 35 & 8 & 0 & -2.462035 & -1.147944 & 1.130612 \\
\hline 36 & 8 & 0 & -2.929860 & 1.034836 & 1.219663 \\
\hline 37 & 6 & 0 & -3.857176 & 3.256897 & 0.795178 \\
\hline 38 & 6 & 0 & -5.496498 & 1.969936 & -1.110020 \\
\hline 39 & 6 & 0 & -4.776532 & 3.975958 & 0.028830 \\
\hline 40 & 1 & 0 & -4.858496 & 5.060288 & 0.173712 \\
\hline 41 & 6 & 0 & -5.603179 & 3.356685 & -0.919873 \\
\hline 42 & 1 & 0 & -6.139547 & 1.480474 & -1.853140 \\
\hline 43 & 1 & 0 & -3.211103 & 3.741306 & 1.534549 \\
\hline 44 & 6 & 0 & -6.608308 & 4.155540 & -1.693898 \\
\hline 45 & 1 & 0 & -6.275873 & 5.198432 & -1.840004 \\
\hline 46 & 1 & 0 & -7.579380 & 4.197109 & -1.163086 \\
\hline 47 & 1 & 0 & -6.802875 & 3.715849 & -2.688308 \\
\hline 48 & 6 & 0 & 4.248168 & 0.812360 & 1.274130 \\
\hline 49 & 6 & 0 & 3.732432 & -0.514737 & 1.816377 \\
\hline 50 & 1 & 0 & 2.914448 & -0.348071 & 2.546981 \\
\hline 51 & 1 & 0 & 4.518187 & -1.081770 & 2.351503 \\
\hline 52 & 1 & 0 & 5.071048 & 0.655093 & 0.548353 \\
\hline 53 & 1 & 0 & 4.647371 & 1.454737 & 2.082418 \\
\hline 54 & 6 & 0 & 3.692517 & 3.161089 & -0.359532 \\
\hline 55 & 6 & 0 & 3.274679 & 4.473040 & -0.104906 \\
\hline 56 & 6 & 0 & 4.715837 & 2.935689 & -1.293171 \\
\hline 57 & 6 & 0 & 3.892668 & 5.541778 & -0.752392 \\
\hline 58 & 1 & 0 & 2.462618 & 4.667240 & 0.606399 \\
\hline 59 & 6 & 0 & 5.334502 & 4.006042 & -1.930541 \\
\hline 60 & 1 & 0 & 5.026237 & 1.909871 & -1.536995 \\
\hline 61 & 6 & 0 & 4.925028 & 5.312094 & -1.659216 \\
\hline 62 & 1 & 0 & 3.563699 & 6.565765 & -0.542380 \\
\hline 63 & 1 & 0 & 6.137909 & 3.819447 & -2.651787 \\
\hline
\end{tabular}




\begin{tabular}{|c|c|c|c|c|c|}
\hline 64 & 1 & 0 & 5.410195 & 6.154846 & -2.163986 \\
\hline 65 & 6 & 0 & 1.825609 & 2.291349 & 1.746338 \\
\hline 66 & 6 & 0 & 2.349145 & 3.068108 & 2.790937 \\
\hline 67 & 6 & 0 & 0.484534 & 1.891120 & 1.797497 \\
\hline 68 & 6 & 0 & 1.542742 & 3.439001 & 3.862733 \\
\hline 69 & 1 & 0 & 3.397420 & 3.395072 & 2.767818 \\
\hline 70 & 6 & 0 & -0.319622 & 2.254655 & 2.877845 \\
\hline 71 & 1 & 0 & 0.066180 & 1.277174 & 0.984100 \\
\hline 72 & 6 & 0 & 0.209463 & 3.028761 & 3.908100 \\
\hline 73 & 1 & 0 & 1.959387 & 4.047682 & 4.673059 \\
\hline 74 & 1 & 0 & -1.361359 & 1.917782 & 2.908998 \\
\hline 75 & 1 & 0 & -0.419872 & 3.312569 & 4.759246 \\
\hline 76 & 6 & 0 & 1.947150 & -2.783296 & 1.266830 \\
\hline 77 & 6 & 0 & 0.601473 & -2.475362 & 1.505910 \\
\hline 78 & 6 & 0 & 2.452881 & -4.034124 & 1.642966 \\
\hline 79 & 6 & 0 & -0.228664 & -3.404543 & 2.127268 \\
\hline 80 & 1 & 0 & 0.187827 & -1.504044 & 1.196962 \\
\hline 81 & 6 & 0 & 1.618174 & -4.960380 & 2.263475 \\
\hline 82 & 1 & 0 & 3.502651 & -4.288196 & 1.446935 \\
\hline 83 & 6 & 0 & 0.280671 & -4.646539 & 2.505741 \\
\hline 84 & 1 & 0 & -1.279675 & -3.148824 & 2.299573 \\
\hline 85 & 1 & 0 & 2.015697 & -5.937917 & 2.558876 \\
\hline 86 & 1 & 0 & -0.372029 & -5.381792 & 2.990194 \\
\hline 87 & 6 & 0 & 4.328019 & -2.421718 & -0.366313 \\
\hline 88 & 6 & 0 & 5.661733 & -2.396603 & 0.056071 \\
\hline 89 & 6 & 0 & 3.978327 & -3.153453 & -1.512114 \\
\hline 90 & 6 & 0 & 6.632554 & -3.102286 & -0.654420 \\
\hline 91 & 1 & 0 & 5.960152 & -1.831491 & 0.947699 \\
\hline 92 & 6 & 0 & 4.948857 & -3.863446 & -2.210564 \\
\hline 93 & 1 & 0 & 2.933091 & -3.161765 & -1.852939 \\
\hline 94 & 6 & 0 & 6.277716 & -3.836614 & -1.783691 \\
\hline 95 & 1 & 0 & 7.675017 & -3.080465 & -0.317572 \\
\hline 96 & 1 & 0 & 4.668814 & -4.437844 & -3.100557 \\
\hline 97 & 1 & 0 & 7.042750 & -4.391223 & -2.338408 \\
\hline
\end{tabular}

Cartesian coordinates (in $\AA$ ) and energy of INT-II-Pd-C(6)-D $\mathrm{E}(\mathrm{UM} 06)=-2961.16652373 \mathrm{au}$

\begin{tabular}{|c|c|c|c|c|c|}
\hline \multirow{2}{*}{$\begin{array}{l}\text { Center } \\
\text { number }\end{array}$} & \multirow{2}{*}{$\begin{array}{l}\text { Atomic } \\
\text { number }\end{array}$} & \multirow{2}{*}{$\begin{array}{l}\text { Atomic } \\
\text { type }\end{array}$} & \multicolumn{3}{|c|}{ Coordinates (Angstroms) } \\
\hline & & & $\mathrm{X}$ & $\mathrm{Y}$ & $\mathrm{Z}$ \\
\hline 1 & 46 & 0 & -0.989744 & -0.171475 & -0.366866 \\
\hline 2 & 15 & 0 & -3.208749 & -0.498720 & 0.310745 \\
\hline 3 & 15 & 0 & -1.168641 & 1.975341 & 0.620101 \\
\hline 4 & 8 & 0 & -0.518706 & -2.486630 & -1.146107 \\
\hline 5 & 8 & 0 & 0.996152 & -0.376944 & -1.622518 \\
\hline 6 & 6 & 0 & 1.542331 & -1.477552 & -1.676551 \\
\hline 7 & 6 & 0 & 0.766060 & -2.704440 & -1.528899 \\
\hline 8 & 6 & 0 & 3.044680 & -1.609099 & -1.816085 \\
\hline 9 & 6 & 0 & 1.317764 & -3.908848 & -1.803203 \\
\hline 10 & 6 & 0 & 2.711014 & -4.058773 & -2.194199 \\
\hline 11 & 1 & 0 & 0.697273 & -4.814908 & -1.734137 \\
\hline 12 & 6 & 0 & 3.182531 & -5.425895 & -2.574374 \\
\hline 13 & 1 & 0 & 2.616388 & -5.822041 & -3.439352 \\
\hline 14 & 1 & 0 & 4.253584 & -5.425084 & -2.836975 \\
\hline 15 & 1 & 0 & 3.038883 & -6.141904 & -1.742107 \\
\hline 16 & 6 & 0 & -2.927313 & 2.088081 & 1.215891 \\
\hline 17 & 6 & 0 & -3.450105 & 0.732698 & 1.678548 \\
\hline 18 & 1 & 0 & -2.886352 & 0.352000 & 2.554342 \\
\hline 19 & 1 & 0 & -4.515397 & 0.787136 & 1.974222 \\
\hline 20 & 1 & 0 & -3.516392 & 2.449829 & 0.348437 \\
\hline 21 & 1 & 0 & -3.015760 & 2.861342 & 2.003534 \\
\hline 22 & 6 & 0 & 3.516036 & -2.977309 & -2.188042 \\
\hline 23 & 1 & 0 & 4.567993 & -3.088487 & -2.470596 \\
\hline 24 & 1 & 0 & -0.980039 & -3.308215 & -0.902894 \\
\hline 25 & 6 & 0 & -0.962442 & 3.372719 & -0.529740 \\
\hline 26 & 6 & 0 & -0.824009 & 3.102975 & -1.896354 \\
\hline 27 & 6 & 0 & -0.968476 & 4.699741 & -0.078835 \\
\hline 28 & 6 & 0 & -0.699022 & 4.152011 & -2.806541 \\
\hline 29 & 1 & 0 & -0.803914 & 2.059790 & -2.245193 \\
\hline
\end{tabular}




\begin{tabular}{|c|c|c|c|c|c|}
\hline 30 & 6 & 0 & -0.840942 & 5.743478 & -0.990775 \\
\hline 31 & 1 & 0 & -1.066803 & 4.918447 & 0.992990 \\
\hline 32 & 6 & 0 & -0.707434 & 5.469802 & -2.353433 \\
\hline 33 & 1 & 0 & -0.588578 & 3.938013 & -3.875395 \\
\hline 34 & 1 & 0 & -0.846360 & 6.780502 & -0.636980 \\
\hline 35 & 1 & 0 & -0.606272 & 6.294519 & -3.067849 \\
\hline 36 & 6 & 0 & -4.515345 & -0.073718 & -0.885867 \\
\hline 37 & 6 & 0 & -5.859884 & 0.045260 & -0.503002 \\
\hline 38 & 6 & 0 & -4.151028 & 0.192411 & -2.210957 \\
\hline 39 & 6 & 0 & -6.822187 & 0.417518 & -1.437057 \\
\hline 40 & 1 & 0 & -6.165233 & -0.161933 & 0.531584 \\
\hline 41 & 6 & 0 & -5.117295 & 0.567199 & -3.143767 \\
\hline 42 & 1 & 0 & -3.096493 & 0.106683 & -2.509487 \\
\hline 43 & 6 & 0 & -6.450741 & 0.679078 & -2.756855 \\
\hline 44 & 1 & 0 & -7.871190 & 0.506495 & -1.133417 \\
\hline 45 & 1 & 0 & -4.825327 & 0.774715 & -4.179066 \\
\hline 46 & 1 & 0 & -7.210383 & 0.974794 & -3.489053 \\
\hline 47 & 6 & 0 & -3.580827 & -2.133922 & 1.017038 \\
\hline 48 & 6 & 0 & -2.672737 & -2.634186 & 1.965219 \\
\hline 49 & 6 & 0 & -4.648615 & -2.936811 & 0.598334 \\
\hline 50 & 6 & 0 & -2.852809 & -3.901262 & 2.510215 \\
\hline 51 & 1 & 0 & -1.806036 & -2.027968 & 2.268323 \\
\hline 52 & 6 & 0 & -4.819491 & -4.209527 & 1.142890 \\
\hline 53 & 1 & 0 & -5.353118 & -2.571031 & -0.158627 \\
\hline 54 & 6 & 0 & -3.929117 & -4.690624 & 2.100703 \\
\hline 55 & 1 & 0 & -2.145574 & -4.280700 & 3.256717 \\
\hline 56 & 1 & 0 & -5.660562 & -4.830239 & 0.814326 \\
\hline 57 & 1 & 0 & -4.070176 & -5.689790 & 2.527598 \\
\hline 58 & 6 & 0 & -0.140462 & 2.389584 & 2.065690 \\
\hline 59 & 6 & 0 & -0.587125 & 2.255542 & 3.386668 \\
\hline 60 & 6 & 0 & 1.195027 & 2.745210 & 1.831223 \\
\hline 61 & 6 & 0 & 0.292002 & 2.470947 & 4.448043 \\
\hline 62 & 1 & 0 & -1.628169 & 1.987052 & 3.606073 \\
\hline 63 & 6 & 0 & 2.071227 & 2.954977 & 2.889903 \\
\hline 64 & 1 & 0 & 1.557107 & 2.850784 & 0.800942 \\
\hline 65 & 6 & 0 & 1.621056 & 2.813008 & 4.203365 \\
\hline 66 & 1 & 0 & -0.067805 & 2.370623 & 5.478169 \\
\hline 67 & 1 & 0 & 3.114332 & 3.224826 & 2.683685 \\
\hline 68 & 1 & 0 & 2.308495 & 2.973567 & 5.041058 \\
\hline 69 & 6 & 0 & 3.685834 & -1.034635 & -0.474773 \\
\hline 70 & 6 & 0 & 3.948730 & 0.457221 & -0.519106 \\
\hline 71 & 6 & 0 & 5.272608 & 0.651127 & -0.142264 \\
\hline 72 & 6 & 0 & 5.122922 & -1.566056 & -0.227059 \\
\hline 73 & 8 & 0 & 5.941607 & -0.527830 & 0.070223 \\
\hline 74 & 8 & 0 & 5.508411 & -2.693212 & -0.230061 \\
\hline 75 & 6 & 0 & 2.845155 & -1.488726 & 0.733421 \\
\hline 76 & 6 & 0 & 1.966224 & -0.603308 & 1.363977 \\
\hline 77 & 6 & 0 & 2.883377 & -2.820386 & 1.171469 \\
\hline 78 & 6 & 0 & 1.128495 & -1.034011 & 2.392504 \\
\hline 79 & 1 & 0 & 1.934823 & 0.446577 & 1.056993 \\
\hline 80 & 6 & 0 & 2.043883 & -3.251780 & 2.196275 \\
\hline 81 & 1 & 0 & 3.584491 & -3.525546 & 0.712261 \\
\hline 82 & 6 & 0 & 1.160368 & -2.363327 & 2.808493 \\
\hline 83 & 1 & 0 & 0.462214 & -0.307824 & 2.878565 \\
\hline 84 & 1 & 0 & 2.096003 & -4.294007 & 2.531483 \\
\hline 85 & 1 & 0 & 0.517731 & -2.702661 & 3.630010 \\
\hline 86 & 6 & 0 & 3.187602 & 1.574173 & -0.863113 \\
\hline 87 & 6 & 0 & 5.865442 & 1.897154 & -0.042784 \\
\hline 88 & 6 & 0 & 5.076524 & 2.999879 & -0.362580 \\
\hline 89 & 1 & 0 & 5.512201 & 4.005590 & -0.303928 \\
\hline 90 & 6 & 0 & 3.747205 & 2.857925 & -0.787072 \\
\hline 91 & 1 & 0 & 2.152960 & 1.452140 & -1.205055 \\
\hline 92 & 1 & 0 & 6.913307 & 1.992953 & 0.257081 \\
\hline 93 & 6 & 0 & 2.940408 & 4.071887 & -1.148118 \\
\hline 94 & 1 & 0 & 2.795955 & 4.741872 & -0.276737 \\
\hline 95 & 1 & 0 & 3.441866 & 4.674629 & -1.928908 \\
\hline 96 & 1 & 0 & 1.938129 & 3.799196 & -1.528376 \\
\hline 97 & 1 & 0 & 3.348936 & -0.900733 & -2.614220 \\
\hline
\end{tabular}


$\mathrm{E}(\mathrm{UM} 06)=-2961.17179395 \mathrm{au}$

\begin{tabular}{|c|c|c|c|c|c|}
\hline \multirow{2}{*}{$\begin{array}{l}\text { Center } \\
\text { number }\end{array}$} & \multirow{2}{*}{$\begin{array}{l}\text { Atomic } \\
\text { number }\end{array}$} & \multirow{2}{*}{$\begin{array}{l}\text { Atomic } \\
\text { type }\end{array}$} & \multicolumn{3}{|c|}{ Coordinates (Angstroms) } \\
\hline & & & $\mathrm{X}$ & $\mathrm{Y}$ & $\mathrm{Z}$ \\
\hline 1 & 46 & 0 & -1.168329 & -0.278553 & -0.242184 \\
\hline 2 & 15 & 0 & -3.454309 & -0.632613 & 0.051552 \\
\hline 3 & 15 & 0 & -1.532300 & 1.856665 & 0.727969 \\
\hline 4 & 8 & 0 & -0.141686 & -2.583623 & -0.776015 \\
\hline 5 & 8 & 0 & 0.847650 & -0.213103 & -1.391050 \\
\hline 6 & 6 & 0 & 1.613322 & -1.173911 & -1.454014 \\
\hline 7 & 6 & 0 & 1.149416 & -2.529020 & -1.179696 \\
\hline 8 & 6 & 0 & 3.074896 & -0.957839 & -1.757061 \\
\hline 9 & 6 & 0 & 1.981525 & -3.583680 & -1.354735 \\
\hline 10 & 6 & 0 & 3.365135 & -3.432591 & -1.781842 \\
\hline 11 & 1 & 0 & 1.607738 & -4.601422 & -1.166934 \\
\hline 12 & 6 & 0 & 4.169702 & -4.680880 & -1.957758 \\
\hline 13 & 1 & 0 & 5.196385 & -4.458458 & -2.294084 \\
\hline 14 & 1 & 0 & 4.236481 & -5.246202 & -1.006914 \\
\hline 15 & 1 & 0 & 3.706110 & -5.359368 & -2.699324 \\
\hline 16 & 6 & 0 & -3.368995 & 1.936352 & 1.066592 \\
\hline 17 & 6 & 0 & -3.977463 & 0.569602 & 1.357965 \\
\hline 18 & 1 & 0 & -3.625061 & 0.174466 & 2.331173 \\
\hline 19 & 1 & 0 & -5.083536 & 0.627883 & 1.401480 \\
\hline 20 & 1 & 0 & -3.824111 & 2.366320 & 0.151498 \\
\hline 21 & 1 & 0 & -3.566969 & 2.645175 & 1.893194 \\
\hline 22 & 6 & 0 & 3.872158 & -2.196574 & -1.972431 \\
\hline 23 & 1 & 0 & 4.919158 & -2.070342 & -2.276665 \\
\hline 24 & 1 & 0 & -0.409115 & -3.482815 & -0.518385 \\
\hline 25 & 6 & 0 & -1.184456 & 3.262799 & -0.370689 \\
\hline 26 & 6 & 0 & -0.266680 & 3.096373 & -1.412615 \\
\hline 27 & 6 & 0 & -1.769160 & 4.517956 & -0.147535 \\
\hline 28 & 6 & 0 & 0.069030 & 4.180714 & -2.222990 \\
\hline 29 & 1 & 0 & 0.208770 & 2.121760 & -1.583159 \\
\hline 30 & 6 & 0 & -1.444486 & 5.590910 & -0.970959 \\
\hline 31 & 1 & 0 & -2.472526 & 4.664877 & 0.682927 \\
\hline 32 & 6 & 0 & -0.522747 & 5.422299 & -2.006790 \\
\hline 33 & 1 & 0 & 0.808551 & 4.048224 & -3.020167 \\
\hline 34 & 1 & 0 & -1.904508 & 6.570260 & -0.797833 \\
\hline 35 & 1 & 0 & -0.260236 & 6.273080 & -2.645754 \\
\hline 36 & 6 & 0 & -4.432779 & -0.122009 & -1.405343 \\
\hline 37 & 6 & 0 & -5.812256 & -0.364480 & -1.462769 \\
\hline 38 & 6 & 0 & -3.805011 & 0.559874 & -2.454449 \\
\hline 39 & 6 & 0 & -6.551601 & 0.074506 & -2.557187 \\
\hline 40 & 1 & 0 & -6.313092 & -0.906866 & -0.649057 \\
\hline 41 & 6 & 0 & -4.550441 & 1.001961 & -3.546430 \\
\hline 42 & 1 & 0 & -2.720799 & 0.742395 & -2.414974 \\
\hline 43 & 6 & 0 & -5.921253 & 0.758258 & -3.597938 \\
\hline 44 & 1 & 0 & -7.629181 & -0.119436 & -2.600202 \\
\hline 45 & 1 & 0 & -4.054462 & 1.535875 & -4.364450 \\
\hline 46 & 1 & 0 & -6.506075 & 1.101083 & -4.458873 \\
\hline 47 & 6 & 0 & -4.128976 & -2.252316 & 0.530838 \\
\hline 48 & 6 & 0 & -4.731340 & -2.503859 & 1.768466 \\
\hline 49 & 6 & 0 & -3.982560 & -3.303169 & -0.388384 \\
\hline 50 & 6 & 0 & -5.183784 & -3.786482 & 2.079361 \\
\hline 51 & 1 & 0 & -4.863506 & -1.700797 & 2.503091 \\
\hline 52 & 6 & 0 & -4.441596 & -4.578479 & -0.077268 \\
\hline 53 & 1 & 0 & -3.516577 & -3.110051 & -1.365949 \\
\hline 54 & 6 & 0 & -5.040677 & -4.822712 & 1.160394 \\
\hline 55 & 1 & 0 & -5.659441 & -3.973276 & 3.048608 \\
\hline 56 & 1 & 0 & -4.337203 & -5.389987 & -0.806421 \\
\hline 57 & 1 & 0 & -5.401708 & -5.827475 & 1.406286 \\
\hline 58 & 6 & 0 & -0.749197 & 2.303240 & 2.314706 \\
\hline 59 & 6 & 0 & -1.217790 & 1.741088 & 3.511242 \\
\hline 60 & 6 & 0 & 0.369845 & 3.145493 & 2.345416 \\
\hline 61 & 6 & 0 & -0.594120 & 2.035760 & 4.720698 \\
\hline 62 & 1 & 0 & -2.082551 & 1.064615 & 3.507549 \\
\hline 63 & 6 & 0 & 0.993976 & 3.432685 & 3.557543 \\
\hline 64 & 1 & 0 & 0.761987 & 3.577721 & 1.417026 \\
\hline 65 & 6 & 0 & 0.514409 & 2.882711 & 4.745340 \\
\hline 66 & 1 & 0 & -0.977302 & 1.602121 & 5.651312 \\
\hline 67 & 1 & 0 & 1.864420 & 4.097964 & 3.572165 \\
\hline
\end{tabular}




$\begin{array}{rrrrrr}68 & 1 & 0 & 1.005928 & 3.115417 & 5.696507 \\ 69 & 6 & 0 & 3.681058 & -0.086981 & -0.583042 \\ 70 & 6 & 0 & 3.346608 & -0.733207 & 0.734424 \\ 71 & 6 & 0 & 2.406087 & 0.070769 & 1.370450 \\ 72 & 6 & 0 & 2.863189 & 1.225534 & -0.488083 \\ 73 & 8 & 0 & 2.154778 & 1.232744 & 0.678778 \\ 74 & 8 & 0 & 2.826237 & 2.124078 & -1.266733 \\ 75 & 6 & 0 & 5.141967 & 0.252042 & -0.835377 \\ 76 & 6 & 0 & 6.123557 & 0.003021 & 0.128025 \\ 77 & 6 & 0 & 5.522382 & 0.854265 & -2.042651 \\ 78 & 6 & 0 & 7.457326 & 0.332039 & -0.113905 \\ 79 & 1 & 0 & 5.848685 & -0.444588 & 1.089370 \\ 80 & 6 & 0 & 6.853067 & 1.180788 & -2.283422 \\ 81 & 1 & 0 & 4.769644 & 1.096332 & -2.801388 \\ 82 & 6 & 0 & 7.827314 & 0.916773 & -1.320865 \\ 83 & 1 & 0 & 8.211659 & 0.129698 & 0.654663 \\ 84 & 1 & 0 & 7.130926 & 1.652728 & -3.232460 \\ 85 & 1 & 0 & 8.875083 & 1.173808 & -1.512034 \\ 86 & 6 & 0 & 3.712360 & -1.945289 & 1.307244 \\ 87 & 6 & 0 & 1.778981 & -0.301658 & 2.546815 \\ 88 & 6 & 0 & 2.147189 & -1.532106 & 3.096052 \\ 89 & 1 & 0 & 1.670499 & -1.859170 & 4.028722 \\ 90 & 6 & 0 & 3.112593 & -2.359546 & 2.505222 \\ 91 & 1 & 0 & 4.464159 & -2.584188 & 0.824912 \\ 92 & 1 & 0 & 1.026213 & 0.340233 & 3.017807 \\ 93 & 6 & 0 & 3.518356 & -3.651525 & 3.150116 \\ 94 & 1 & 0 & 4.394977 & -3.515123 & 3.813155 \\ 95 & 1 & 0 & 2.707425 & -4.070747 & 3.771941 \\ 96 & 1 & 0 & 3.800979 & -4.411152 & 2.398167 \\ 97 & 1 & 0 & 3.110129 & -0.315692 & -2.662785 \\ ----------------------------------------------------------------------------\end{array}$

Cartesian coordinates (in $\AA$ ) and energy of INT-II-Pd-C(6)-F $\mathrm{E}(\mathrm{UM} 06)=-2961.17351498 \mathrm{au}$

\begin{tabular}{|c|c|c|c|c|c|}
\hline \multirow{2}{*}{$\begin{array}{l}\text { Center } \\
\text { number }\end{array}$} & \multirow{2}{*}{$\begin{array}{l}\text { Atomic } \\
\text { number }\end{array}$} & \multirow{2}{*}{$\begin{array}{l}\text { Atomic } \\
\text { type }\end{array}$} & \multicolumn{3}{|c|}{ Coordinates (Angstroms) } \\
\hline & & & $\mathrm{X}$ & $\mathrm{Y}$ & $\mathrm{Z}$ \\
\hline 1 & 46 & 0 & -1.353592 & -0.230062 & -0.560838 \\
\hline 2 & 15 & 0 & -3.475169 & -0.812173 & 0.263580 \\
\hline 3 & 15 & 0 & -1.684592 & 1.851502 & 0.503051 \\
\hline 4 & 8 & 0 & -0.585306 & -2.479841 & -1.227016 \\
\hline 5 & 8 & 0 & 0.741881 & -0.226296 & -1.636630 \\
\hline 6 & 6 & 0 & 1.402077 & -1.257741 & -1.560916 \\
\hline 7 & 6 & 0 & 0.756333 & -2.563842 & -1.392046 \\
\hline 8 & 6 & 0 & 2.916624 & -1.229476 & -1.610800 \\
\hline 9 & 6 & 0 & 1.468573 & -3.711781 & -1.417393 \\
\hline 10 & 6 & 0 & 2.913391 & -3.732256 & -1.589213 \\
\hline 11 & 1 & 0 & 0.945793 & -4.672665 & -1.302155 \\
\hline 12 & 6 & 0 & 3.582311 & -5.067444 & -1.657242 \\
\hline 13 & 1 & 0 & 4.674106 & -4.969380 & -1.779563 \\
\hline 14 & 1 & 0 & 3.392069 & -5.654914 & -0.738452 \\
\hline 15 & 1 & 0 & 3.194549 & -5.665298 & -2.504482 \\
\hline 16 & 6 & 0 & -3.450957 & 1.826650 & 1.083898 \\
\hline 17 & 6 & 0 & -3.810742 & 0.437709 & 1.595367 \\
\hline 18 & 1 & 0 & -3.186589 & 0.163357 & 2.469753 \\
\hline 19 & 1 & 0 & -4.866575 & 0.384799 & 1.922516 \\
\hline 20 & 1 & 0 & -4.088631 & 2.104047 & 0.219172 \\
\hline 21 & 1 & 0 & -3.604019 & 2.589043 & 1.872586 \\
\hline 22 & 6 & 0 & 3.582894 & -2.566710 & -1.685079 \\
\hline 23 & 1 & 0 & 4.671759 & -2.572844 & -1.819341 \\
\hline 24 & 1 & 0 & -0.976114 & -3.320775 & -0.931055 \\
\hline 25 & 6 & 0 & -1.494381 & 3.402060 & -0.426259 \\
\hline 26 & 6 & 0 & -0.472137 & 3.449074 & -1.382767 \\
\hline 27 & 6 & 0 & -2.333689 & 4.511494 & -0.253409 \\
\hline 28 & 6 & 0 & -0.287834 & 4.597645 & -2.149222 \\
\hline 29 & 1 & 0 & 0.178215 & 2.574250 & -1.532379 \\
\hline 30 & 6 & 0 & -2.145229 & 5.656228 & -1.023969 \\
\hline 31 & 1 & 0 & -3.145943 & 4.489897 & 0.484221 \\
\hline 32 & 6 & 0 & -1.122749 & 5.699452 & -1.971685 \\
\hline 33 & 1 & 0 & 0.511801 & 4.627082 & -2.898581 \\
\hline
\end{tabular}




\begin{tabular}{|c|c|c|c|c|c|}
\hline 34 & 1 & 0 & -2.804706 & 6.520366 & -0.886124 \\
\hline 35 & 1 & 0 & -0.981373 & 6.598847 & -2.581604 \\
\hline 36 & 6 & 0 & -4.892156 & -0.650379 & -0.870706 \\
\hline 37 & 6 & 0 & -6.217981 & -0.667955 & -0.412323 \\
\hline 38 & 6 & 0 & -4.638317 & -0.470649 & -2.235355 \\
\hline 39 & 6 & 0 & -7.269869 & -0.514757 & -1.310615 \\
\hline 40 & 1 & 0 & -6.436790 & -0.813867 & 0.654161 \\
\hline 41 & 6 & 0 & -5.694242 & -0.316482 & -3.132756 \\
\hline 42 & 1 & 0 & -3.599801 & -0.446129 & -2.593912 \\
\hline 43 & 6 & 0 & -7.007876 & -0.338803 & -2.670529 \\
\hline 44 & 1 & 0 & -8.303660 & -0.531853 & -0.947821 \\
\hline 45 & 1 & 0 & -5.487818 & -0.174683 & -4.199261 \\
\hline 46 & 1 & 0 & -7.838457 & -0.216073 & -3.374650 \\
\hline 47 & 6 & 0 & -3.583379 & -2.444083 & 1.065749 \\
\hline 48 & 6 & 0 & -2.585235 & -2.763848 & 2.001606 \\
\hline 49 & 6 & 0 & -4.542279 & -3.407766 & 0.730402 \\
\hline 50 & 6 & 0 & -2.571007 & -4.011797 & 2.616174 \\
\hline 51 & 1 & 0 & -1.803758 & -2.029580 & 2.246404 \\
\hline 52 & 6 & 0 & -4.517684 & -4.660483 & 1.343296 \\
\hline 53 & 1 & 0 & -5.316395 & -3.182472 & -0.013418 \\
\hline 54 & 6 & 0 & -3.539559 & -4.962327 & 2.288627 \\
\hline 55 & 1 & 0 & -1.795224 & -4.246641 & 3.353558 \\
\hline 56 & 1 & 0 & -5.275451 & -5.406829 & 1.079538 \\
\hline 57 & 1 & 0 & -3.527740 & -5.946127 & 2.770896 \\
\hline 58 & 6 & 0 & -0.749776 & 2.030614 & 2.065853 \\
\hline 59 & 6 & 0 & -0.074827 & 0.916410 & 2.575215 \\
\hline 60 & 6 & 0 & -0.718142 & 3.241075 & 2.770394 \\
\hline 61 & 6 & 0 & 0.617699 & 1.006109 & 3.782334 \\
\hline 62 & 1 & 0 & -0.053477 & -0.022219 & 2.002428 \\
\hline 63 & 6 & 0 & -0.022251 & 3.329375 & 3.973442 \\
\hline 64 & 1 & 0 & -1.231048 & 4.127425 & 2.372574 \\
\hline 65 & 6 & 0 & 0.645341 & 2.212100 & 4.480401 \\
\hline 66 & 1 & 0 & 1.151432 & 0.128640 & 4.166651 \\
\hline 67 & 1 & 0 & 0.002059 & 4.278502 & 4.520837 \\
\hline 68 & 1 & 0 & 1.193558 & 2.286496 & 5.426427 \\
\hline 69 & 6 & 0 & 3.454500 & -0.403450 & -0.390405 \\
\hline 70 & 6 & 0 & 4.946882 & -0.518049 & -0.190479 \\
\hline 71 & 6 & 0 & 5.160287 & -1.109065 & 1.047039 \\
\hline 72 & 6 & 0 & 2.941733 & -1.111199 & 0.880425 \\
\hline 73 & 8 & 0 & 3.976320 & -1.446615 & 1.677789 \\
\hline 74 & 8 & 0 & 1.804280 & -1.367861 & 1.155542 \\
\hline 75 & 6 & 0 & 3.069621 & 1.071365 & -0.400020 \\
\hline 76 & 6 & 0 & 2.661002 & 1.710187 & 0.772590 \\
\hline 77 & 6 & 0 & 3.269992 & 1.849148 & -1.547419 \\
\hline 78 & 6 & 0 & 2.453050 & 3.089667 & 0.803568 \\
\hline 79 & 1 & 0 & 2.509593 & 1.140183 & 1.697177 \\
\hline 80 & 6 & 0 & 3.072776 & 3.225396 & -1.516922 \\
\hline 81 & 1 & 0 & 3.589713 & 1.383839 & -2.488504 \\
\hline 82 & 6 & 0 & 2.661511 & 3.853008 & -0.339896 \\
\hline 83 & 1 & 0 & 2.128119 & 3.561929 & 1.738701 \\
\hline 84 & 1 & 0 & 3.243553 & 3.815642 & -2.425071 \\
\hline 85 & 1 & 0 & 2.501912 & 4.937078 & -0.319061 \\
\hline 86 & 6 & 0 & 6.033525 & -0.126356 & -0.959467 \\
\hline 87 & 6 & 0 & 6.420975 & -1.339228 & 1.562702 \\
\hline 88 & 6 & 0 & 7.503195 & -0.946676 & 0.773277 \\
\hline 89 & 1 & 0 & 8.521445 & -1.116042 & 1.144870 \\
\hline 90 & 6 & 0 & 7.334632 & -0.341499 & -0.481236 \\
\hline 91 & 1 & 0 & 5.887307 & 0.351613 & -1.937715 \\
\hline 92 & 1 & 0 & 6.554631 & -1.811555 & 2.540478 \\
\hline 93 & 6 & 0 & 8.524898 & 0.098304 & -1.279890 \\
\hline 94 & 1 & 0 & 8.842439 & 1.120484 & -0.995479 \\
\hline 95 & 1 & 0 & 9.392595 & -0.565386 & -1.116982 \\
\hline 96 & 1 & 0 & 8.307277 & 0.116226 & -2.362546 \\
\hline 97 & 1 & 0 & 3.184744 & -0.650410 & -2.519255 \\
\hline
\end{tabular}

Cartesian coordinates (in $\AA$ ) and energy of INT-I-Pd-C(5)-A $\mathrm{E}(\mathrm{UM} 06)=-2961.17865543 \mathrm{au}$

\begin{tabular}{lllcc} 
Center & Atomic & Atomic & \multicolumn{2}{c}{ Coordinates (Angstroms) } \\
number & number & type & $X$ & $Y$
\end{tabular}




\begin{tabular}{|c|c|c|c|c|c|}
\hline 1 & 46 & 0 & 0.487488 & 0.103013 & -0.330353 \\
\hline 2 & 15 & 0 & 2.431207 & -1.017000 & 0.050743 \\
\hline 3 & 15 & 0 & 1.729357 & 2.011932 & 0.107570 \\
\hline 4 & 8 & 0 & -0.462234 & -1.653224 & -0.825070 \\
\hline 5 & 8 & 0 & -1.327371 & 0.923244 & -1.259187 \\
\hline 6 & 6 & 0 & -2.061668 & -0.156684 & -1.785807 \\
\hline 7 & 6 & 0 & -1.549817 & -1.445882 & -1.548811 \\
\hline 8 & 6 & 0 & -3.224289 & 0.062369 & -2.508777 \\
\hline 9 & 6 & 0 & -2.280926 & -2.524071 & -2.084308 \\
\hline 10 & 6 & 0 & -3.473959 & -2.334364 & -2.778786 \\
\hline 11 & 1 & 0 & -1.903101 & -3.536169 & -1.891646 \\
\hline 12 & 1 & 0 & -1.935314 & 1.542389 & -0.794610 \\
\hline 13 & 1 & 0 & -3.573357 & 1.091203 & -2.669499 \\
\hline 14 & 6 & 0 & -3.936533 & -1.026407 & -2.999140 \\
\hline 15 & 1 & 0 & -4.866562 & -0.865465 & -3.556755 \\
\hline 16 & 6 & 0 & -4.283773 & -3.509927 & -3.231249 \\
\hline 17 & 1 & 0 & -4.842937 & -3.289676 & -4.158308 \\
\hline 18 & 1 & 0 & -5.026711 & -3.779834 & -2.455105 \\
\hline 19 & 1 & 0 & -3.654022 & -4.399558 & -3.409166 \\
\hline 20 & 6 & 0 & -3.446371 & -0.559396 & 1.060232 \\
\hline 21 & 6 & 0 & -2.284856 & -0.923852 & 1.802379 \\
\hline 22 & 6 & 0 & -2.253165 & -2.339589 & 1.796802 \\
\hline 23 & 6 & 0 & -3.974130 & 0.735132 & 0.697070 \\
\hline 24 & 6 & 0 & -3.235665 & 1.920471 & 0.928425 \\
\hline 25 & 6 & 0 & -5.223836 & 0.867876 & 0.049024 \\
\hline 26 & 6 & 0 & -3.702544 & 3.161200 & 0.502903 \\
\hline 27 & 1 & 0 & -2.280850 & 1.868391 & 1.458946 \\
\hline 28 & 6 & 0 & -5.691334 & 2.112380 & -0.354547 \\
\hline 29 & 1 & 0 & -5.818954 & -0.028872 & -0.141118 \\
\hline 30 & 6 & 0 & -4.934197 & 3.266059 & -0.145379 \\
\hline 31 & 1 & 0 & -3.107807 & 4.063756 & 0.695730 \\
\hline 32 & 1 & 0 & -6.666694 & 2.182591 & -0.849079 \\
\hline 33 & 1 & 0 & -5.306505 & 4.242770 & -0.473103 \\
\hline 34 & 6 & 0 & -4.073420 & -1.831395 & 0.639060 \\
\hline 35 & 8 & 0 & -5.064691 & -2.060461 & 0.004801 \\
\hline 36 & 8 & 0 & -3.287607 & -2.870213 & 1.114168 \\
\hline 37 & 6 & 0 & -1.281814 & -3.080436 & 2.441453 \\
\hline 38 & 6 & 0 & -1.253978 & -0.249333 & 2.496606 \\
\hline 39 & 6 & 0 & -0.285715 & -2.380416 & 3.117458 \\
\hline 40 & 1 & 0 & 0.496296 & -2.938086 & 3.650501 \\
\hline 41 & 6 & 0 & -0.260572 & -0.971204 & 3.154747 \\
\hline 42 & 1 & 0 & -1.235576 & 0.843049 & 2.583461 \\
\hline 43 & 1 & 0 & -1.314589 & -4.174164 & 2.419261 \\
\hline 44 & 6 & 0 & 0.809856 & -0.273987 & 3.940865 \\
\hline 45 & 1 & 0 & 0.709422 & -0.472873 & 5.025686 \\
\hline 46 & 1 & 0 & 0.775066 & 0.822745 & 3.803812 \\
\hline 47 & 1 & 0 & 1.821485 & -0.631133 & 3.655901 \\
\hline 48 & 6 & 0 & 3.501657 & 1.493065 & 0.211270 \\
\hline 49 & 6 & 0 & 3.599152 & 0.138298 & 0.894692 \\
\hline 50 & 1 & 0 & 3.288367 & 0.203000 & 1.957043 \\
\hline 51 & 1 & 0 & 4.629056 & -0.267123 & 0.866653 \\
\hline 52 & 1 & 0 & 3.854039 & 1.430765 & -0.838387 \\
\hline 53 & 1 & 0 & 4.114417 & 2.270309 & 0.706173 \\
\hline 54 & 6 & 0 & 1.642287 & 3.274183 & -1.190852 \\
\hline 55 & 6 & 0 & 2.004502 & 4.601569 & -0.922341 \\
\hline 56 & 6 & 0 & 1.273923 & 2.895185 & -2.487730 \\
\hline 57 & 6 & 0 & 2.002525 & 5.538828 & -1.951140 \\
\hline 58 & 1 & 0 & 2.286416 & 4.906228 & 0.094171 \\
\hline 59 & 6 & 0 & 1.273499 & 3.839623 & -3.511540 \\
\hline 60 & 1 & 0 & 0.973636 & 1.859621 & -2.694093 \\
\hline 61 & 6 & 0 & 1.637814 & 5.158066 & -3.243112 \\
\hline 62 & 1 & 0 & 2.286078 & 6.576310 & -1.743025 \\
\hline 63 & 1 & 0 & 0.979950 & 3.544550 & -4.524687 \\
\hline 64 & 1 & 0 & 1.633578 & 5.900356 & -4.048980 \\
\hline 65 & 6 & 0 & 1.310769 & 2.846468 & 1.665138 \\
\hline 66 & 6 & 0 & 2.188295 & 2.949077 & 2.749029 \\
\hline 67 & 6 & 0 & 0.016007 & 3.374683 & 1.773124 \\
\hline 68 & 6 & 0 & 1.770372 & 3.565813 & 3.928756 \\
\hline 69 & 1 & 0 & 3.209105 & 2.551944 & 2.690520 \\
\hline 70 & 6 & 0 & -0.400328 & 3.981933 & 2.952683 \\
\hline 71 & 1 & 0 & -0.668219 & 3.320257 & 0.913639 \\
\hline
\end{tabular}




\begin{tabular}{|c|c|c|c|c|c|}
\hline 72 & 6 & 0 & 0.477752 & 4.073909 & 4.034672 \\
\hline 73 & 1 & 0 & 2.463678 & 3.647578 & 4.772927 \\
\hline 74 & 1 & 0 & -1.414827 & 4.389874 & 3.030344 \\
\hline 75 & 1 & 0 & 0.151771 & 4.551930 & 4.964768 \\
\hline 76 & 6 & 0 & 2.391394 & -2.566201 & 0.974121 \\
\hline 77 & 6 & 0 & 1.604758 & -3.604966 & 0.454839 \\
\hline 78 & 6 & 0 & 3.157057 & -2.767139 & 2.130132 \\
\hline 79 & 6 & 0 & 1.601576 & -4.843081 & 1.089086 \\
\hline 80 & 1 & 0 & 0.979046 & -3.423391 & -0.428453 \\
\hline 81 & 6 & 0 & 3.143467 & -4.010909 & 2.757879 \\
\hline 82 & 1 & 0 & 3.781686 & -1.966816 & 2.545763 \\
\hline 83 & 6 & 0 & 2.369361 & -5.047212 & 2.236246 \\
\hline 84 & 1 & 0 & 0.990794 & -5.657725 & 0.684412 \\
\hline 85 & 1 & 0 & 3.749821 & -4.173813 & 3.655686 \\
\hline 86 & 1 & 0 & 2.367454 & -6.026105 & 2.728685 \\
\hline 87 & 6 & 0 & 3.186185 & -1.402562 & -1.558942 \\
\hline 88 & 6 & 0 & 4.463924 & -1.980548 & -1.583677 \\
\hline 89 & 6 & 0 & 2.508951 & -1.153583 & -2.757642 \\
\hline 90 & 6 & 0 & 5.065841 & -2.280839 & -2.801016 \\
\hline 91 & 1 & 0 & 4.990317 & -2.213639 & -0.647844 \\
\hline 92 & 6 & 0 & 3.116156 & -1.462557 & -3.974051 \\
\hline 93 & 1 & 0 & 1.491862 & -0.738832 & -2.738858 \\
\hline 94 & 6 & 0 & 4.392764 & -2.019182 & -3.995603 \\
\hline 95 & 1 & 0 & 6.064484 & -2.730737 & -2.818768 \\
\hline 96 & 1 & 0 & 2.581734 & -1.273689 & -4.911305 \\
\hline 97 & 1 & 0 & 4.867072 & -2.262063 & -4.952882 \\
\hline
\end{tabular}

Cartesian coordinates (in Å) and energy of INT-I-Pd-C(5)-B

$\mathrm{E}(\mathrm{UM} 06)=-2961.19270371 \mathrm{au}$

\begin{tabular}{|c|c|c|c|c|c|}
\hline \multirow{2}{*}{$\begin{array}{l}\text { Center } \\
\text { number }\end{array}$} & \multirow{2}{*}{$\begin{array}{l}\text { Atomic } \\
\text { number }\end{array}$} & \multirow{2}{*}{$\begin{array}{l}\text { Atomic } \\
\text { type }\end{array}$} & \multicolumn{3}{|c|}{ Coordinates (Angstroms) } \\
\hline & & & $X$ & $\mathrm{Y}$ & $\mathrm{Z}$ \\
\hline 1 & 46 & 0 & -0.851635 & 0.018279 & -0.351907 \\
\hline 2 & 15 & 0 & -2.243212 & 1.691679 & 0.312141 \\
\hline 3 & 15 & 0 & -2.570982 & -1.391800 & 0.294221 \\
\hline 4 & 8 & 0 & 0.595809 & 1.342788 & -0.987419 \\
\hline 5 & 8 & 0 & 0.484637 & -1.381026 & -1.339496 \\
\hline 6 & 6 & 0 & 1.578272 & -0.657854 & -1.839952 \\
\hline 7 & 6 & 0 & 1.582585 & 0.733693 & -1.612885 \\
\hline 8 & 6 & 0 & 2.596461 & -1.301195 & -2.532530 \\
\hline 9 & 6 & 0 & 2.709740 & 1.445730 & -2.072002 \\
\hline 10 & 6 & 0 & 3.767667 & 0.813119 & -2.720855 \\
\hline 11 & 1 & 0 & 2.735438 & 2.526715 & -1.883740 \\
\hline 12 & 1 & 0 & 0.828672 & -2.121763 & -0.766482 \\
\hline 13 & 1 & 0 & 2.530261 & -2.383840 & -2.696448 \\
\hline 14 & 6 & 0 & 3.697217 & -0.571073 & -2.961988 \\
\hline 15 & 1 & 0 & 4.517317 & -1.074088 & -3.489350 \\
\hline 16 & 6 & 0 & 4.984229 & 1.578121 & -3.146540 \\
\hline 17 & 1 & 0 & 5.886085 & 1.203225 & -2.621166 \\
\hline 18 & 1 & 0 & 4.890203 & 2.656938 & -2.930807 \\
\hline 19 & 1 & 0 & 5.176446 & 1.464130 & -4.230270 \\
\hline 20 & 6 & 0 & 3.168494 & -0.892894 & 0.923805 \\
\hline 21 & 6 & 0 & 4.500632 & -0.560006 & 0.550139 \\
\hline 22 & 6 & 0 & 5.002628 & -1.686180 & -0.137873 \\
\hline 23 & 6 & 0 & 2.189993 & -0.158633 & 1.694977 \\
\hline 24 & 6 & 0 & 2.248565 & 1.245757 & 1.821351 \\
\hline 25 & 6 & 0 & 1.160144 & -0.839944 & 2.378411 \\
\hline 26 & 6 & 0 & 1.356269 & 1.926064 & 2.638090 \\
\hline 27 & 1 & 0 & 2.990602 & 1.810315 & 1.247512 \\
\hline 28 & 6 & 0 & 0.263992 & -0.152318 & 3.190790 \\
\hline 29 & 1 & 0 & 1.098410 & -1.929969 & 2.308778 \\
\hline 30 & 6 & 0 & 0.365930 & 1.231301 & 3.336450 \\
\hline 31 & 1 & 0 & 1.426958 & 3.016437 & 2.730085 \\
\hline 32 & 1 & 0 & -0.500827 & -0.716566 & 3.740637 \\
\hline 33 & 1 & 0 & -0.316819 & 1.773513 & 4.002071 \\
\hline 34 & 6 & 0 & 2.930965 & -2.229532 & 0.379796 \\
\hline 35 & 8 & 0 & 1.912424 & -2.902147 & 0.337004 \\
\hline 36 & 8 & 0 & 4.074869 & -2.680648 & -0.214078 \\
\hline 37 & 6 & 0 & 6.281190 & -1.753134 & -0.653542 \\
\hline
\end{tabular}




\begin{tabular}{|c|c|c|c|c|c|}
\hline 38 & 6 & 0 & 5.356174 & 0.546429 & 0.733644 \\
\hline 39 & 6 & 0 & 7.090112 & -0.631012 & -0.476097 \\
\hline 40 & 1 & 0 & 8.113686 & -0.642585 & -0.872307 \\
\hline 41 & 6 & 0 & 6.645536 & 0.519622 & 0.214043 \\
\hline 42 & 1 & 0 & 5.024216 & 1.426812 & 1.294658 \\
\hline 43 & 1 & 0 & 6.632131 & -2.649756 & -1.173236 \\
\hline 44 & 6 & 0 & 7.567435 & 1.692760 & 0.361646 \\
\hline 45 & 1 & 0 & 7.738751 & 2.192830 & -0.611861 \\
\hline 46 & 1 & 0 & 8.559303 & 1.384775 & 0.741101 \\
\hline 47 & 1 & 0 & 7.159924 & 2.448586 & 1.055227 \\
\hline 48 & 6 & 0 & -4.022797 & -0.332502 & 0.732712 \\
\hline 49 & 6 & 0 & -3.540489 & 0.949063 & 1.395467 \\
\hline 50 & 1 & 0 & -3.066760 & 0.742468 & 2.376651 \\
\hline 51 & 1 & 0 & -4.368070 & 1.666099 & 1.559525 \\
\hline 52 & 1 & 0 & -4.531736 & -0.110732 & -0.227098 \\
\hline 53 & 1 & 0 & -4.747926 & -0.890924 & 1.354471 \\
\hline 54 & 6 & 0 & -3.132407 & -2.528190 & -1.001269 \\
\hline 55 & 6 & 0 & -3.968363 & -3.604955 & -0.673405 \\
\hline 56 & 6 & 0 & -2.769162 & -2.300524 & -2.333494 \\
\hline 57 & 6 & 0 & -4.444062 & -4.440148 & -1.679102 \\
\hline 58 & 1 & 0 & -4.243679 & -3.796539 & 0.372394 \\
\hline 59 & 6 & 0 & -3.246523 & -3.144154 & -3.334501 \\
\hline 60 & 1 & 0 & -2.096232 & -1.470842 & -2.585994 \\
\hline 61 & 6 & 0 & -4.083104 & -4.209532 & -3.007500 \\
\hline 62 & 1 & 0 & -5.097209 & -5.282008 & -1.424690 \\
\hline 63 & 1 & 0 & -2.955895 & -2.971913 & -4.376420 \\
\hline 64 & 1 & 0 & -4.454211 & -4.873957 & -3.795722 \\
\hline 65 & 6 & 0 & -2.176361 & -2.415883 & 1.738269 \\
\hline 66 & 6 & 0 & -2.836206 & -2.303893 & 2.967394 \\
\hline 67 & 6 & 0 & -1.130088 & -3.336983 & 1.592843 \\
\hline 68 & 6 & 0 & -2.448202 & -3.106329 & 4.039875 \\
\hline 69 & 1 & 0 & -3.660786 & -1.593714 & 3.105755 \\
\hline 70 & 6 & 0 & -0.744616 & -4.132681 & 2.666025 \\
\hline 71 & 1 & 0 & -0.596959 & -3.431552 & 0.637505 \\
\hline 72 & 6 & 0 & -1.402053 & -4.015716 & 3.891543 \\
\hline 73 & 1 & 0 & -2.970243 & -3.020286 & 4.999222 \\
\hline 74 & 1 & 0 & 0.081105 & -4.841997 & 2.541559 \\
\hline 75 & 1 & 0 & -1.098199 & -4.641303 & 4.738012 \\
\hline 76 & 6 & 0 & -1.552270 & 3.135878 & 1.145478 \\
\hline 77 & 6 & 0 & -0.562664 & 3.862523 & 0.467867 \\
\hline 78 & 6 & 0 & -2.012238 & 3.563467 & 2.396537 \\
\hline 79 & 6 & 0 & -0.046187 & 5.018080 & 1.045825 \\
\hline 80 & 1 & 0 & -0.185810 & 3.500660 & -0.496984 \\
\hline 81 & 6 & 0 & -1.486941 & 4.721195 & 2.966527 \\
\hline 82 & 1 & 0 & -2.785090 & 3.004838 & 2.938625 \\
\hline 83 & 6 & 0 & -0.507084 & 5.447940 & 2.291080 \\
\hline 84 & 1 & 0 & 0.724939 & 5.590347 & 0.518416 \\
\hline 85 & 1 & 0 & -1.851520 & 5.061793 & 3.941904 \\
\hline 86 & 1 & 0 & -0.100566 & 6.361457 & 2.739377 \\
\hline 87 & 6 & 0 & -3.121210 & 2.330873 & -1.147677 \\
\hline 88 & 6 & 0 & -4.131982 & 3.286299 & -0.967642 \\
\hline 89 & 6 & 0 & -2.789697 & 1.900046 & -2.436501 \\
\hline 90 & 6 & 0 & -4.820121 & 3.783970 & -2.069045 \\
\hline 91 & 1 & 0 & -4.376847 & 3.658125 & 0.036829 \\
\hline 92 & 6 & 0 & -3.479188 & 2.407695 & -3.536948 \\
\hline 93 & 1 & 0 & -1.972035 & 1.180881 & -2.579670 \\
\hline 94 & 6 & 0 & -4.495218 & 3.342369 & -3.352923 \\
\hline 95 & 1 & 0 & -5.610970 & 4.528522 & -1.926708 \\
\hline 96 & 1 & 0 & -3.214159 & 2.073818 & -4.545956 \\
\hline 97 & 1 & 0 & -5.035669 & 3.739958 & -4.219081 \\
\hline
\end{tabular}

Cartesian coordinates (in $\AA$ ) and energy of INT-I-Pd-C(5)-C $\mathrm{E}(\mathrm{UM} 06)=-2961.17946549 \mathrm{au}$

\begin{tabular}{cccccc}
$\begin{array}{l}\text { Center } \\
\text { number }\end{array}$ & $\begin{array}{c}\text { Atomic } \\
\text { number }\end{array}$ & $\begin{array}{c}\text { Atomic } \\
\text { type }\end{array}$ & $\mathrm{X}$ & \multicolumn{2}{c}{ Coordinates (Angstroms) } \\
\hline 1 & 46 & 0 & 1.056475 & -0.138275 & -0.513167 \\
2 & 15 & 0 & 2.634536 & -1.622927 & 0.168570 \\
3 & 15 & 0 & 2.621206 & 1.458396 & 0.033796
\end{tabular}




\begin{tabular}{|c|c|c|c|c|c|}
\hline 4 & 8 & 0 & -0.282774 & -1.642122 & -0.934770 \\
\hline 5 & 8 & 0 & -0.484740 & 1.020218 & -1.535028 \\
\hline 6 & 6 & 0 & -1.570361 & 0.167627 & -1.815387 \\
\hline 7 & 6 & 0 & -1.393200 & -1.190974 & -1.486680 \\
\hline 8 & 6 & 0 & -2.722410 & 0.648059 & -2.414466 \\
\hline 9 & 6 & 0 & -2.474063 & -2.048152 & -1.766155 \\
\hline 10 & 6 & 0 & -3.655829 & -1.587532 & -2.348943 \\
\hline 11 & 1 & 0 & -2.359818 & -3.106269 & -1.497995 \\
\hline 12 & 1 & 0 & -0.797817 & 1.860268 & -1.139456 \\
\hline 13 & 1 & 0 & -2.793972 & 1.713744 & -2.670741 \\
\hline 14 & 6 & 0 & -3.772335 & -0.229410 & -2.678799 \\
\hline 15 & 1 & 0 & -4.688424 & 0.140452 & -3.155662 \\
\hline 16 & 6 & 0 & -4.791496 & -2.537718 & -2.586114 \\
\hline 17 & 1 & 0 & -5.556901 & -2.100937 & -3.252418 \\
\hline 18 & 1 & 0 & -5.294909 & -2.801320 & -1.634222 \\
\hline 19 & 1 & 0 & -4.442796 & -3.483550 & -3.040268 \\
\hline 20 & 6 & 0 & -3.693461 & 0.145093 & 1.061448 \\
\hline 21 & 6 & 0 & -3.384654 & 1.505944 & 0.743971 \\
\hline 22 & 6 & 0 & -2.023627 & 1.686744 & 1.070677 \\
\hline 23 & 6 & 0 & -4.903355 & -0.609179 & 0.868485 \\
\hline 24 & 6 & 0 & -5.914321 & -0.151875 & -0.008643 \\
\hline 25 & 6 & 0 & -5.113202 & -1.840622 & 1.533831 \\
\hline 26 & 6 & 0 & -7.082960 & -0.874937 & -0.193791 \\
\hline 27 & 1 & 0 & -5.759459 & 0.762491 & -0.587150 \\
\hline 28 & 6 & 0 & -6.288837 & -2.554573 & 1.348683 \\
\hline 29 & 1 & 0 & -4.342422 & -2.222612 & 2.208135 \\
\hline 30 & 6 & 0 & -7.281226 & -2.077041 & 0.489759 \\
\hline 31 & 1 & 0 & -7.848004 & -0.503391 & -0.884720 \\
\hline 32 & 1 & 0 & -6.437151 & -3.498262 & 1.885299 \\
\hline 33 & 1 & 0 & -8.207754 & -2.644694 & 0.348036 \\
\hline 34 & 6 & 0 & -2.462668 & -0.427480 & 1.628962 \\
\hline 35 & 8 & 0 & -2.201629 & -1.509509 & 2.076943 \\
\hline 36 & 8 & 0 & -1.471365 & 0.559155 & 1.583798 \\
\hline 37 & 6 & 0 & -1.346756 & 2.875161 & 0.858684 \\
\hline 38 & 6 & 0 & -4.094278 & 2.611952 & 0.239922 \\
\hline 39 & 6 & 0 & -2.073639 & 3.938017 & 0.309254 \\
\hline 40 & 1 & 0 & -1.568112 & 4.895569 & 0.129270 \\
\hline 41 & 6 & 0 & -3.445910 & 3.827119 & 0.014727 \\
\hline 42 & 1 & 0 & -5.166344 & 2.541784 & 0.028613 \\
\hline 43 & 1 & 0 & -0.290673 & 2.978536 & 1.137953 \\
\hline 44 & 6 & 0 & -4.198874 & 5.017681 & -0.500271 \\
\hline 45 & 1 & 0 & -3.611859 & 5.576847 & -1.251490 \\
\hline 46 & 1 & 0 & -4.434510 & 5.727029 & 0.316572 \\
\hline 47 & 1 & 0 & -5.156344 & 4.726305 & -0.965733 \\
\hline 48 & 6 & 0 & 4.264540 & 0.606813 & -0.045056 \\
\hline 49 & 6 & 0 & 4.121625 & -0.685436 & 0.748083 \\
\hline 50 & 1 & 0 & 3.943543 & -0.471495 & 1.821032 \\
\hline 51 & 1 & 0 & 5.025961 & -1.320686 & 0.698767 \\
\hline 52 & 1 & 0 & 4.498669 & 0.411637 & -1.110421 \\
\hline 53 & 1 & 0 & 5.060379 & 1.260456 & 0.360099 \\
\hline 54 & 6 & 0 & 2.626106 & 2.868382 & -1.104457 \\
\hline 55 & 6 & 0 & 2.391732 & 4.183532 & -0.683904 \\
\hline 56 & 6 & 0 & 2.781300 & 2.592121 & -2.471722 \\
\hline 57 & 6 & 0 & 2.344114 & 5.213439 & -1.621922 \\
\hline 58 & 1 & 0 & 2.249014 & 4.410647 & 0.379858 \\
\hline 59 & 6 & 0 & 2.740318 & 3.625695 & -3.400978 \\
\hline 60 & 1 & 0 & 2.917000 & 1.557631 & -2.817051 \\
\hline 61 & 6 & 0 & 2.524143 & 4.937253 & -2.975854 \\
\hline 62 & 1 & 0 & 2.167854 & 6.242333 & -1.289374 \\
\hline 63 & 1 & 0 & 2.867558 & 3.405517 & -4.466339 \\
\hline 64 & 1 & 0 & 2.488392 & 5.750187 & -3.709447 \\
\hline 65 & 6 & 0 & 2.537758 & 2.062632 & 1.744995 \\
\hline 66 & 6 & 0 & 3.508962 & 2.953209 & 2.230006 \\
\hline 67 & 6 & 0 & 1.544059 & 1.580181 & 2.605745 \\
\hline 68 & 6 & 0 & 3.471859 & 3.366078 & 3.557928 \\
\hline 69 & 1 & 0 & 4.299187 & 3.333237 & 1.568207 \\
\hline 70 & 6 & 0 & 1.513571 & 1.998917 & 3.935930 \\
\hline 71 & 1 & 0 & 0.783471 & 0.877708 & 2.238041 \\
\hline 72 & 6 & 0 & 2.473008 & 2.890320 & 4.409807 \\
\hline 73 & 1 & 0 & 4.229207 & 4.063127 & 3.933105 \\
\hline 74 & 1 & 0 & 0.732830 & 1.619859 & 4.604094 \\
\hline 75 & 1 & 0 & 2.447641 & 3.217236 & 5.455250 \\
\hline
\end{tabular}




\begin{tabular}{|c|c|c|c|c|c|}
\hline 76 & 6 & 0 & 2.098010 & -2.708206 & 1.505249 \\
\hline 77 & 6 & 0 & 0.829951 & -2.554256 & 2.072652 \\
\hline 78 & 6 & 0 & 2.975269 & -3.698903 & 1.972006 \\
\hline 79 & 6 & 0 & 0.443708 & -3.387958 & 3.120800 \\
\hline 80 & 1 & 0 & 0.120307 & -1.812239 & 1.685333 \\
\hline 81 & 6 & 0 & 2.582823 & -4.519047 & 3.023687 \\
\hline 82 & 1 & 0 & 3.962044 & -3.837253 & 1.508586 \\
\hline 83 & 6 & 0 & 1.318439 & -4.361372 & 3.597347 \\
\hline 84 & 1 & 0 & -0.556962 & -3.268389 & 3.549569 \\
\hline 85 & 1 & 0 & 3.263455 & -5.293272 & 3.394600 \\
\hline 86 & 1 & 0 & 1.012110 & -5.015942 & 4.421192 \\
\hline 87 & 6 & 0 & 3.143177 & -2.649950 & -1.233489 \\
\hline 88 & 6 & 0 & 4.447139 & -2.645890 & -1.744502 \\
\hline 89 & 6 & 0 & 2.163288 & -3.474203 & -1.808281 \\
\hline 90 & 6 & 0 & 4.772132 & -3.472128 & -2.818431 \\
\hline 91 & 1 & 0 & 5.227088 & -2.007190 & -1.312370 \\
\hline 92 & 6 & 0 & 2.499846 & -4.296571 & -2.878014 \\
\hline 93 & 1 & 0 & 1.134165 & -3.443144 & -1.426572 \\
\hline 94 & 6 & 0 & 3.801180 & -4.297544 & -3.382114 \\
\hline 95 & 1 & 0 & 5.793778 & -3.472632 & -3.213869 \\
\hline 96 & 1 & 0 & 1.736277 & -4.941241 & -3.326548 \\
\hline 97 & 1 & 0 & 4.060801 & -4.947460 & -4.225132 \\
\hline
\end{tabular}

Cartesian coordinates (in ̊̊) and energy of INT-I-Pd-C(5)-D $\mathrm{E}(\mathrm{UM} 06)=-2961.18649200 \mathrm{au}$

\begin{tabular}{|c|c|c|c|c|c|}
\hline \multirow{2}{*}{$\begin{array}{l}\text { Center } \\
\text { number }\end{array}$} & \multirow{2}{*}{$\begin{array}{l}\text { Atomic } \\
\text { number }\end{array}$} & \multirow{2}{*}{$\begin{array}{l}\text { Atomic } \\
\text { type }\end{array}$} & \multicolumn{3}{|c|}{ Coordinates (Angstroms) } \\
\hline & & & $\mathrm{X}$ & $\mathrm{Y}$ & $\mathrm{Z}$ \\
\hline 1 & 8 & 0 & 0.973433 & -0.077440 & 1.964703 \\
\hline 2 & 8 & 0 & 0.690737 & 0.853031 & -1.365102 \\
\hline 3 & 8 & 0 & 0.350033 & -1.774944 & -0.628125 \\
\hline 4 & 6 & 0 & 2.133846 & -0.224448 & 1.644780 \\
\hline 5 & 6 & 0 & 3.083512 & 0.714708 & 1.040644 \\
\hline 6 & 6 & 0 & 4.287824 & -0.016132 & 0.857731 \\
\hline 7 & 6 & 0 & 5.578155 & 0.267505 & 0.361807 \\
\hline 8 & 1 & 0 & 5.838364 & 1.273789 & 0.014238 \\
\hline 9 & 6 & 0 & 6.546157 & -0.730590 & 0.334215 \\
\hline 10 & 6 & 0 & 6.224999 & -2.016867 & 0.821564 \\
\hline 11 & 1 & 0 & 7.000636 & -2.793512 & 0.802342 \\
\hline 12 & 6 & 0 & 4.965623 & -2.328877 & 1.336528 \\
\hline 13 & 1 & 0 & 4.724134 & -3.323204 & 1.724033 \\
\hline 14 & 6 & 0 & 4.026178 & -1.319428 & 1.339962 \\
\hline 15 & 6 & 0 & 3.814310 & -0.665881 & -2.620486 \\
\hline 16 & 6 & 0 & 2.824757 & 0.277617 & -2.376618 \\
\hline 17 & 1 & 0 & 2.924135 & 1.312102 & -2.728239 \\
\hline 18 & 6 & 0 & 1.690782 & -0.086787 & -1.662191 \\
\hline 19 & 6 & 0 & 1.450086 & -1.414940 & -1.250070 \\
\hline 20 & 6 & 0 & 2.474019 & -2.348367 & -1.522667 \\
\hline 21 & 6 & 0 & 3.648232 & -1.992295 & -2.179538 \\
\hline 22 & 6 & 0 & 2.733104 & 2.079268 & 0.705715 \\
\hline 23 & 6 & 0 & 1.656986 & 2.731763 & 1.350338 \\
\hline 24 & 1 & 0 & 1.120936 & 2.213280 & 2.149108 \\
\hline 25 & 6 & 0 & 1.296909 & 4.030514 & 1.008243 \\
\hline 26 & 1 & 0 & 0.473907 & 4.518865 & 1.547347 \\
\hline 27 & 6 & 0 & 1.988432 & 4.715575 & 0.007252 \\
\hline 28 & 1 & 0 & 1.705655 & 5.739852 & -0.261980 \\
\hline 29 & 6 & 0 & 3.056594 & 4.089834 & -0.639049 \\
\hline 30 & 1 & 0 & 3.608709 & 4.619363 & -1.423626 \\
\hline 31 & 6 & 0 & 3.428794 & 2.795173 & -0.292998 \\
\hline 32 & 1 & 0 & 4.252369 & 2.311717 & -0.827670 \\
\hline 33 & 6 & 0 & 7.915846 & -0.471505 & -0.217851 \\
\hline 34 & 1 & 0 & 8.073620 & -1.015792 & -1.169859 \\
\hline 35 & 1 & 0 & 8.705623 & -0.810798 & 0.477713 \\
\hline 36 & 1 & 0 & 8.080587 & 0.601308 & -0.418600 \\
\hline 37 & 1 & 0 & 2.316013 & -3.377553 & -1.175765 \\
\hline 38 & 6 & 0 & 4.730837 & -2.998475 & -2.427119 \\
\hline 39 & 1 & 0 & 5.704944 & -2.634128 & -2.044546 \\
\hline 40 & 1 & 0 & 4.514600 & -3.963689 & -1.936078 \\
\hline 41 & 1 & 0 & 4.863962 & -3.192857 & -3.508983 \\
\hline
\end{tabular}




\begin{tabular}{|c|c|c|c|c|c|}
\hline 42 & 8 & 0 & 2.754773 & -1.434979 & 1.809869 \\
\hline 43 & 1 & 0 & 4.723302 & -0.382782 & -3.166021 \\
\hline 44 & 46 & 0 & -0.923958 & -0.179582 & -0.293874 \\
\hline 45 & 15 & 0 & -2.643693 & -1.521027 & 0.321519 \\
\hline 46 & 15 & 0 & -2.416763 & 1.538644 & -0.000971 \\
\hline 47 & 6 & 0 & -4.035466 & -0.454352 & 0.907629 \\
\hline 48 & 6 & 0 & -4.115842 & 0.797883 & 0.046188 \\
\hline 49 & 1 & 0 & -3.794394 & -0.199987 & 1.959533 \\
\hline 50 & 1 & 0 & -4.983493 & -1.025204 & 0.931213 \\
\hline 51 & 1 & 0 & -4.833613 & 1.538338 & 0.448065 \\
\hline 52 & 1 & 0 & -4.424006 & 0.558388 & -0.989765 \\
\hline 53 & 6 & 0 & -3.224040 & -2.419595 & -1.143237 \\
\hline 54 & 6 & 0 & -4.551223 & -2.378589 & -1.588274 \\
\hline 55 & 6 & 0 & -2.277476 & -3.201310 & -1.823334 \\
\hline 56 & 6 & 0 & -4.930285 & -3.120345 & -2.706005 \\
\hline 57 & 1 & 0 & -5.308218 & -1.778968 & -1.067802 \\
\hline 58 & 6 & 0 & -2.667647 & -3.940447 & -2.935213 \\
\hline 59 & 1 & 0 & -1.232598 & -3.207299 & -1.481364 \\
\hline 60 & 6 & 0 & -3.990881 & -3.900815 & -3.376830 \\
\hline 61 & 1 & 0 & -5.969801 & -3.090719 & -3.050652 \\
\hline 62 & 1 & 0 & -1.929305 & -4.551579 & -3.465613 \\
\hline 63 & 1 & 0 & -4.292956 & -4.483898 & -4.253926 \\
\hline 64 & 6 & 0 & -2.287330 & -2.735345 & 1.607771 \\
\hline 65 & 6 & 0 & -1.140494 & -2.616789 & 2.400304 \\
\hline 66 & 6 & 0 & -3.200400 & -3.779248 & 1.819012 \\
\hline 67 & 6 & 0 & -0.920309 & -3.546458 & 3.416107 \\
\hline 68 & 1 & 0 & -0.409569 & -1.816990 & 2.216865 \\
\hline 69 & 6 & 0 & -2.971303 & -4.695308 & 2.839383 \\
\hline 70 & 1 & 0 & -4.088519 & -3.882816 & 1.180705 \\
\hline 71 & 6 & 0 & -1.831215 & -4.577198 & 3.636726 \\
\hline 72 & 1 & 0 & -0.019531 & -3.464719 & 4.033983 \\
\hline 73 & 1 & 0 & -3.681602 & -5.511758 & 3.009737 \\
\hline 74 & 1 & 0 & -1.649210 & -5.305334 & 4.435246 \\
\hline 75 & 6 & 0 & -2.289642 & 2.456248 & 1.559347 \\
\hline 76 & 6 & 0 & -1.494615 & 1.946475 & 2.592418 \\
\hline 77 & 6 & 0 & -3.039645 & 3.625630 & 1.761720 \\
\hline 78 & 6 & 0 & -1.437890 & 2.615017 & 3.815720 \\
\hline 79 & 1 & 0 & -0.900527 & 1.035525 & 2.436952 \\
\hline 80 & 6 & 0 & -2.971252 & 4.289541 & 2.982262 \\
\hline 81 & 1 & 0 & -3.677483 & 4.024574 & 0.961388 \\
\hline 82 & 6 & 0 & -2.168000 & 3.785607 & 4.007847 \\
\hline 83 & 1 & 0 & -0.813475 & 2.215557 & 4.622611 \\
\hline 84 & 1 & 0 & -3.552132 & 5.205366 & 3.137704 \\
\hline 85 & 1 & 0 & -2.117762 & 4.310947 & 4.968136 \\
\hline 86 & 6 & 0 & -2.306770 & 2.690242 & -1.392494 \\
\hline 87 & 6 & 0 & -2.710578 & 2.245404 & -2.660189 \\
\hline 88 & 6 & 0 & -1.677555 & 3.934311 & -1.261358 \\
\hline 89 & 6 & 0 & -2.525663 & 3.056898 & -3.774353 \\
\hline 90 & 1 & 0 & -3.156099 & 1.248957 & -2.787371 \\
\hline 91 & 6 & 0 & -1.486777 & 4.737939 & -2.383555 \\
\hline 92 & 1 & 0 & -1.333874 & 4.279931 & -0.278687 \\
\hline 93 & 6 & 0 & -1.915795 & 4.304378 & -3.636399 \\
\hline 94 & 1 & 0 & -2.850197 & 2.708737 & -4.760824 \\
\hline 95 & 1 & 0 & -0.999541 & 5.713533 & -2.275959 \\
\hline 96 & 1 & 0 & -1.767498 & 4.940019 & -4.516288 \\
\hline 97 & 1 & 0 & 1.083651 & 1.671787 & -0.996402 \\
\hline
\end{tabular}

Cartesian coordinates (in $\AA$ ) and energy of INT-I-Pd-C(5)-E $\mathrm{E}(\mathrm{UM} 06)=-2961.18179089 \mathrm{au}$

\begin{tabular}{cccccc}
$\begin{array}{c}\text { Center } \\
\text { number }\end{array}$ & $\begin{array}{c}\text { Atomic } \\
\text { number }\end{array}$ & $\begin{array}{c}\text { Atomic } \\
\text { type }\end{array}$ & $\mathrm{X}$ & \multicolumn{3}{c}{$\begin{array}{c}\text { Coordinates (Angstroms) } \\
\text { Y }\end{array}$} & \\
\hline 1 & 8 & 0 & 2.856663 & 2.487491 & -1.150267 \\
2 & 8 & 0 & 0.498590 & -1.538778 & 0.845768 \\
3 & 1 & 0 & 0.832903 & -1.968283 & 0.023323 \\
4 & 8 & 0 & 0.588527 & 1.221085 & 0.862291 \\
5 & 6 & 0 & 3.617665 & 1.597529 & -0.879753 \\
6 & 6 & 0 & 3.544874 & 0.138714 & -1.114115 \\
7 & 6 & 0 & 4.770029 & -0.394159 & -0.622266
\end{tabular}




\begin{tabular}{|c|c|c|c|c|c|}
\hline 8 & 6 & 0 & 5.387704 & -1.664456 & -0.570133 \\
\hline 9 & 1 & 0 & 4.913751 & -2.548361 & -1.008687 \\
\hline 10 & 6 & 0 & 6.638787 & -1.814505 & 0.016140 \\
\hline 11 & 6 & 0 & 7.291427 & -0.682006 & 0.551557 \\
\hline 12 & 1 & 0 & 8.280860 & -0.812128 & 1.008548 \\
\hline 13 & 6 & 0 & 6.729813 & 0.591935 & 0.49508 \\
\hline 14 & 1 & 0 & 7.243231 & 1.474332 & 0.88961 \\
\hline 15 & 6 & 0 & 5.484567 & 0.706405 & -0.09162 \\
\hline 16 & 6 & 0 & 3.646888 & -0.904478 & 2.68506 \\
\hline 17 & 6 & 0 & 2.584696 & -1.585332 & 2.09394 \\
\hline 18 & 1 & 0 & 2.537948 & -2.681540 & 2.09743 \\
\hline 19 & 6 & 0 & 1.570916 & -0.866481 & 1.481325 \\
\hline 20 & 6 & 0 & 1.557544 & 0.542387 & $1.4407 \mathrm{C}$ \\
\hline 21 & 6 & 0 & 2.645398 & 1.199307 & 2.0488 \\
\hline 22 & 6 & 0 & 3.679035 & 0.498511 & 2.6714 \\
\hline 23 & 6 & 0 & 2.398854 & -0.515420 & -1.705665 \\
\hline 24 & 6 & 0 & 1.345402 & 0.225955 & -2.28808 \\
\hline 25 & 1 & 0 & 1.406902 & 1.317565 & -2.296374 \\
\hline 26 & 6 & 0 & 0.244878 & -0.411505 & -2.855426 \\
\hline 27 & 1 & 0 & -0.546607 & 0.198036 & -3.313420 \\
\hline 28 & 6 & 0 & 0.153183 & -1.803670 & -2.871415 \\
\hline 29 & 1 & 0 & -0.701568 & -2.303320 & -3.342412 \\
\hline 30 & 6 & 0 & 1.173877 & -2.556107 & -2.283595 \\
\hline 31 & 1 & 0 & 1.120077 & -3.651888 & -2.286644 \\
\hline 32 & 6 & 0 & 2.271850 & -1.925623 & -1.704718 \\
\hline 33 & 1 & 0 & 3.045957 & -2.540999 & -1.238614 \\
\hline 34 & 6 & 0 & 7.315751 & -3.151034 & 0.07529 \\
\hline 35 & 1 & 0 & 6.690648 & -3.947084 & -0.365320 \\
\hline 36 & 1 & 0 & 7.546084 & -3.441959 & 1.117761 \\
\hline 37 & 1 & 0 & 8.277657 & -3.140427 & -0.471593 \\
\hline 38 & 1 & 0 & 2.657749 & 2.296365 & 2.00975 \\
\hline 39 & 6 & 0 & 4.791409 & 1.248888 & 3.34117 \\
\hline 40 & 1 & 0 & 5.055400 & 2.163759 & 2.778703 \\
\hline 41 & 1 & 0 & 4.498734 & 1.569984 & 4.360190 \\
\hline 42 & 1 & 0 & 5.699693 & 0.627622 & 3.441955 \\
\hline 43 & 8 & 0 & 4.811268 & 1.870861 & -0.242404 \\
\hline 44 & 1 & 0 & 4.457484 & -1.465028 & 3.166079 \\
\hline 45 & 46 & 0 & -0.947629 & -0.007715 & 0.239675 \\
\hline 46 & 15 & 0 & -2.216294 & 1.765501 & -0.403913 \\
\hline 47 & 15 & 0 & -2.871364 & -1.259298 & -0.017632 \\
\hline 48 & 6 & 0 & -3.765049 & 1.096733 & -1.162484 \\
\hline 49 & 6 & 0 & -4.260430 & -0.073315 & -0.328704 \\
\hline 50 & 1 & 0 & -4.524767 & 1.899200 & -1.239451 \\
\hline 51 & 1 & 0 & -3.501818 & 0.777576 & -2.191533 \\
\hline 52 & 1 & 0 & -5.126365 & -0.588424 & -0.787143 \\
\hline 53 & 1 & 0 & -4.585357 & 0.270009 & 0.674601 \\
\hline 54 & 6 & 0 & -2.726335 & 2.753606 & 1.028124 \\
\hline 55 & 6 & 0 & -3.374079 & 3.978393 & 0.812577 \\
\hline 56 & 6 & 0 & -2.498745 & 2.299860 & 2.331607 \\
\hline 57 & 6 & 0 & -3.806988 & 4.731367 & 1.899012 \\
\hline 58 & 1 & 0 & -3.530978 & 4.353196 & -0.208164 \\
\hline 59 & 6 & 0 & -2.933602 & 3.060674 & 3.414946 \\
\hline 60 & 1 & 0 & -1.958734 & 1.358415 & 2.497300 \\
\hline 61 & 6 & 0 & -3.588069 & 4.271472 & 3.198492 \\
\hline 62 & 1 & 0 & -4.311371 & 5.689231 & 1.731577 \\
\hline 63 & 1 & 0 & -2.749400 & 2.709197 & 4.435827 \\
\hline 64 & 1 & 0 & -3.923967 & 4.870680 & 4.052048 \\
\hline 65 & 6 & 0 & -1.454979 & 2.866994 & -1.613466 \\
\hline 66 & 6 & 0 & -2.144408 & 3.294098 & -2.759191 \\
\hline 67 & 6 & 0 & -0.147968 & 3.310390 & -1.366112 \\
\hline 68 & 6 & 0 & -1.526027 & 4.171176 & -3.645975 \\
\hline 69 & 1 & 0 & -3.167856 & 2.959247 & -2.967883 \\
\hline 70 & 6 & 0 & 0.462825 & 4.178885 & -2.267288 \\
\hline 71 & 1 & 0 & 0.408605 & 2.944177 & -0.492084 \\
\hline 72 & 6 & 0 & -0.224917 & 4.611572 & -3.400029 \\
\hline 73 & 1 & 0 & -2.064378 & 4.511220 & -4.537516 \\
\hline 74 & 1 & 0 & 1.494223 & 4.494429 & -2.078901 \\
\hline 75 & 1 & 0 & 0.259394 & 5.295463 & -4.106104 \\
\hline 76 & 6 & 0 & -3.292584 & -2.144927 & 1.509553 \\
\hline 77 & 6 & 0 & -2.537516 & -1.946371 & 2.671123 \\
\hline 78 & 6 & 0 & -4.405494 & -2.998004 & 1.532661 \\
\hline 79 & 5 & 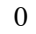 & -2.897426 & -2.595963 & 3.8 \\
\hline
\end{tabular}




$\begin{array}{rrrrrr}80 & 1 & 0 & -1.656007 & -1.290955 & 2.652891 \\ 81 & 6 & 0 & -4.761993 & -3.637970 & 2.715022 \\ 82 & 1 & 0 & -4.993415 & -3.168695 & 0.620464 \\ 83 & 6 & 0 & -4.007807 & -3.436834 & 3.872642 \\ 84 & 1 & 0 & -2.301834 & -2.445728 & 4.757833 \\ 85 & 1 & 0 & -5.632571 & -4.302676 & 2.734233 \\ 86 & 1 & 0 & -4.288701 & -3.946531 & 4.800968 \\ 87 & 6 & 0 & -2.898517 & -2.506755 & -1.335235 \\ 88 & 6 & 0 & -3.575082 & -2.311871 & -2.544148 \\ 89 & 6 & 0 & -2.163835 & -3.683372 & -1.130949 \\ 90 & 6 & 0 & -3.524986 & -3.290787 & -3.535843 \\ 91 & 1 & 0 & -4.159129 & -1.400865 & -2.724836 \\ 92 & 6 & 0 & -2.123537 & -4.660196 & -2.120616 \\ 93 & 1 & 0 & -1.629461 & -3.839095 & -0.182729 \\ 94 & 6 & 0 & -2.802240 & -4.463840 & -3.324610 \\ 95 & 1 & 0 & -4.063222 & -3.138447 & -4.477709 \\ 96 & 1 & 0 & -1.561221 & -5.585305 & -1.951260 \\ 97 & 1 & 0 & -2.771708 & -5.234613 & -4.102503\end{array}$

Cartesian coordinates (in ̊̊) and energy of INT-I-Pd-C(5)-F $\mathrm{E}(\mathrm{UM} 06)=-2961.15540263 \mathrm{au}$

\begin{tabular}{|c|c|c|c|c|c|}
\hline \multirow{2}{*}{$\begin{array}{l}\text { Center } \\
\text { number }\end{array}$} & \multirow{2}{*}{$\begin{array}{l}\text { Atomic } \\
\text { number }\end{array}$} & \multirow{2}{*}{$\begin{array}{l}\text { Atomic } \\
\text { type }\end{array}$} & \multicolumn{3}{|c|}{ Coordinates (Angstroms) } \\
\hline & & & $\mathrm{X}$ & $\mathrm{Y}$ & $\mathrm{Z}$ \\
\hline 1 & 8 & 0 & -5.011853 & -3.157024 & 0.780126 \\
\hline 2 & 8 & 0 & -0.554632 & -1.843496 & -1.227516 \\
\hline 3 & 1 & 0 & -0.653268 & -2.805285 & -1.110264 \\
\hline 4 & 8 & 0 & -0.630713 & 0.836871 & -1.413901 \\
\hline 5 & 6 & 0 & -4.367809 & -2.162141 & 0.901007 \\
\hline 6 & 6 & 0 & -4.722235 & -0.723468 & 0.704181 \\
\hline 7 & 6 & 0 & -3.549204 & 0.001115 & 0.974131 \\
\hline 8 & 6 & 0 & -3.164194 & 1.364169 & 0.873711 \\
\hline 9 & 1 & 0 & -3.882163 & 2.118644 & 0.532660 \\
\hline 10 & 6 & 0 & -1.872798 & 1.749109 & 1.197630 \\
\hline 11 & 6 & 0 & -0.955730 & 0.763997 & 1.636058 \\
\hline 12 & 1 & 0 & 0.043404 & 1.091818 & 1.952454 \\
\hline 13 & 6 & 0 & -1.279433 & -0.592389 & 1.705544 \\
\hline 14 & 1 & 0 & -0.552723 & -1.341007 & 2.038631 \\
\hline 15 & 6 & 0 & -2.556019 & -0.951753 & 1.322684 \\
\hline 16 & 6 & 0 & -4.108016 & -1.230384 & -1.927940 \\
\hline 17 & 6 & 0 & -2.920593 & -1.937380 & -1.675719 \\
\hline 18 & 1 & 0 & -2.950343 & -3.028596 & -1.554037 \\
\hline 19 & 6 & 0 & -1.741397 & -1.253593 & -1.502738 \\
\hline 20 & 6 & 0 & -1.690229 & 0.196207 & -1.637017 \\
\hline 21 & 6 & 0 & -2.909575 & 0.846531 & -2.024901 \\
\hline 22 & 6 & 0 & -4.096049 & 0.164960 & -2.191340 \\
\hline 23 & 6 & 0 & -6.091085 & -0.270695 & 0.559523 \\
\hline 24 & 6 & 0 & -7.087202 & -1.112615 & 0.021653 \\
\hline 25 & 1 & 0 & -6.828752 & -2.134422 & -0.272375 \\
\hline 26 & 6 & 0 & -8.387246 & -0.653311 & -0.138401 \\
\hline 27 & 1 & 0 & -9.145993 & -1.316778 & -0.567512 \\
\hline 28 & 6 & 0 & -8.732164 & 0.643151 & 0.249208 \\
\hline 29 & 1 & 0 & -9.760530 & 0.999332 & 0.123170 \\
\hline 30 & 6 & 0 & -7.767748 & 1.475879 & 0.816675 \\
\hline 31 & 1 & 0 & -8.038998 & 2.482345 & 1.153522 \\
\hline 32 & 6 & 0 & -6.462488 & 1.025433 & 0.971373 \\
\hline 33 & 1 & 0 & -5.727705 & 1.672215 & 1.461828 \\
\hline 34 & 6 & 0 & -1.408108 & 3.161743 & 1.029850 \\
\hline 35 & 1 & 0 & -0.767425 & 3.244048 & 0.127800 \\
\hline 36 & 1 & 0 & -0.799008 & 3.499353 & 1.890085 \\
\hline 37 & 1 & 0 & -2.253648 & 3.861669 & 0.909337 \\
\hline 38 & 1 & 0 & -2.845391 & 1.929707 & -2.192183 \\
\hline 39 & 6 & 0 & -5.356803 & 0.872066 & -2.578610 \\
\hline 40 & 1 & 0 & -5.935801 & 1.183424 & -1.683956 \\
\hline 41 & 1 & 0 & -5.142490 & 1.783021 & -3.164552 \\
\hline 42 & 1 & 0 & -6.021952 & 0.223658 & -3.176908 \\
\hline 43 & 8 & 0 & -3.032813 & -2.216515 & 1.295985 \\
\hline 44 & 1 & 0 & -5.040650 & -1.793412 & -2.058911 \\
\hline 45 & 46 & 0 & 1.187425 & -0.113727 & -0.458484 \\
\hline
\end{tabular}




\begin{tabular}{|c|c|c|c|c|c|}
\hline 46 & 15 & 0 & 2.649916 & 1.733834 & -0.020671 \\
\hline 47 & 15 & 0 & 3.032044 & -1.470887 & -0.022319 \\
\hline 48 & 6 & 0 & 4.298038 & 0.975899 & 0.407373 \\
\hline 49 & 6 & 0 & 4.493539 & -0.365474 & -0.284652 \\
\hline 50 & 1 & 0 & 4.314742 & 0.860553 & 1.508233 \\
\hline 51 & 1 & 0 & 5.113368 & 1.679472 & 0.154789 \\
\hline 52 & 1 & 0 & 5.432486 & -0.863643 & 0.027474 \\
\hline 53 & 1 & 0 & 4.557508 & -0.235231 & -1.384642 \\
\hline 54 & 6 & 0 & 2.938413 & 2.796503 & -1.472782 \\
\hline 55 & 6 & 0 & 4.064642 & 3.624657 & -1.584267 \\
\hline 56 & 6 & 0 & 1.974379 & 2.797965 & -2.489064 \\
\hline 57 & 6 & 0 & 4.228626 & 4.433586 & -2.704709 \\
\hline 58 & 1 & 0 & 4.816056 & 3.657169 & -0.784051 \\
\hline 59 & 6 & 0 & 2.142425 & 3.613888 & -3.607867 \\
\hline 60 & 1 & 0 & 1.082549 & 2.162137 & -2.397510 \\
\hline 61 & 6 & 0 & 3.268470 & 4.426011 & -3.717983 \\
\hline 62 & 1 & 0 & 5.110742 & 5.078310 & -2.787472 \\
\hline 63 & 1 & 0 & 1.386389 & 3.611669 & -4.400854 \\
\hline 64 & 1 & 0 & 3.401097 & 5.063079 & -4.599708 \\
\hline 65 & 6 & 0 & 2.327751 & 2.859308 & 1.378941 \\
\hline 66 & 6 & 0 & 2.243150 & 2.308628 & 2.668342 \\
\hline 67 & 6 & 0 & 2.060464 & 4.221512 & 1.199183 \\
\hline 68 & 6 & 0 & 1.923840 & 3.111931 & 3.758877 \\
\hline 69 & 1 & 0 & 2.422457 & 1.233885 & 2.825207 \\
\hline 70 & 6 & 0 & 1.738243 & 5.022087 & 2.294623 \\
\hline 71 & 1 & 0 & 2.107650 & 4.665457 & 0.196830 \\
\hline 72 & 6 & 0 & 1.671577 & 4.472360 & 3.573525 \\
\hline 73 & 1 & 0 & 1.870186 & 2.673526 & 4.761730 \\
\hline 74 & 1 & 0 & 1.540722 & 6.089610 & 2.145267 \\
\hline 75 & 1 & 0 & 1.421506 & 5.105854 & 4.431773 \\
\hline 76 & 6 & 0 & 3.167570 & -2.130703 & 1.672154 \\
\hline 77 & 6 & 0 & 2.084469 & -2.889683 & 2.140389 \\
\hline 78 & 6 & 0 & 4.264463 & -1.917362 & 2.514071 \\
\hline 79 & 6 & 0 & 2.099700 & -3.430359 & 3.421716 \\
\hline 80 & 1 & 0 & 1.222631 & -3.066300 & 1.479261 \\
\hline 81 & 6 & 0 & 4.272872 & -2.449975 & 3.804081 \\
\hline 82 & 1 & 0 & 5.132796 & -1.341205 & 2.171116 \\
\hline 83 & 6 & 0 & 3.194371 & -3.204994 & 4.258407 \\
\hline 84 & 1 & 0 & 1.252771 & -4.030379 & 3.772933 \\
\hline 85 & 1 & 0 & 5.136392 & -2.277858 & 4.456290 \\
\hline 86 & 1 & 0 & 3.206302 & -3.624199 & 5.270551 \\
\hline 87 & 6 & 0 & 3.330650 & -2.895530 & -1.119845 \\
\hline 88 & 6 & 0 & 2.793171 & -2.851633 & -2.413161 \\
\hline 89 & 6 & 0 & 4.094611 & -3.999729 & -0.721431 \\
\hline 90 & 6 & 0 & 3.029932 & -3.895898 & -3.303983 \\
\hline 91 & 1 & 0 & 2.179439 & -1.991285 & -2.717623 \\
\hline 92 & 6 & 0 & 4.322250 & -5.044456 & -1.614102 \\
\hline 93 & 1 & 0 & 4.513041 & -4.046685 & 0.292570 \\
\hline 94 & 6 & 0 & 3.792796 & -4.992502 & -2.903539 \\
\hline 95 & 1 & 0 & 2.611812 & -3.856408 & -4.316064 \\
\hline 96 & 1 & 0 & 4.919929 & -5.907634 & -1.300461 \\
\hline 97 & 1 & 0 & 3.974237 & -5.817097 & -3.602006 \\
\hline
\end{tabular}

Cartesian coordinates (in $\AA$ ) and energy of TS-Pd-C(5)-A $\mathrm{E}(\mathrm{UM} 06)=-2961.15075168 \mathrm{au}$

\begin{tabular}{|c|c|c|c|c|c|}
\hline \multirow{2}{*}{$\begin{array}{l}\text { Center } \\
\text { number }\end{array}$} & \multirow{2}{*}{$\begin{array}{l}\text { Atomic } \\
\text { number }\end{array}$} & \multirow{2}{*}{$\begin{array}{l}\text { Atomic } \\
\text { type }\end{array}$} & \multicolumn{3}{|c|}{ Coordinates (Angstroms) } \\
\hline & & & $\mathrm{X}$ & $\mathrm{Y}$ & $\mathrm{Z}$ \\
\hline 1 & 46 & 0 & 1.219872 & 0.148895 & -0.497838 \\
\hline 2 & 15 & 0 & 2.621705 & -1.532314 & 0.455900 \\
\hline 3 & 15 & 0 & 2.835834 & 1.675182 & 0.216719 \\
\hline 4 & 8 & 0 & -0.392518 & -1.221844 & -1.227673 \\
\hline 5 & 8 & 0 & -0.913985 & 1.412914 & -1.314793 \\
\hline 6 & 6 & 0 & -1.915796 & 0.554573 & -1.621489 \\
\hline 7 & 6 & 0 & -1.530383 & -0.861398 & -1.595000 \\
\hline 8 & 6 & 0 & -3.186671 & 0.925647 & -1.951436 \\
\hline 9 & 6 & 0 & -2.514509 & -1.805426 & -2.050745 \\
\hline 10 & 6 & 0 & -3.778334 & -1.431181 & -2.427864 \\
\hline 11 & 1 & 0 & -2.178900 & -2.846618 & -2.134055 \\
\hline
\end{tabular}




\begin{tabular}{|c|c|c|c|c|c|}
\hline 12 & 1 & 0 & -1.209123 & 2.336893 & -1.389149 \\
\hline 13 & 1 & 0 & -3.482506 & 1.984191 & -1.934243 \\
\hline 14 & 6 & 0 & -4.169782 & -0.067979 & -2.205315 \\
\hline 15 & 1 & 0 & -5.153303 & 0.253207 & -2.569821 \\
\hline 16 & 6 & 0 & -4.728622 & -2.391367 & -3.066840 \\
\hline 17 & 1 & 0 & -4.788603 & -2.193946 & -4.155329 \\
\hline 18 & 1 & 0 & -5.756472 & -2.299900 & -2.670608 \\
\hline 19 & 1 & 0 & -4.397130 & -3.435409 & -2.933173 \\
\hline 20 & 6 & 0 & -4.964897 & -0.135395 & 0.022245 \\
\hline 21 & 6 & 0 & -3.767935 & -0.488257 & 0.713196 \\
\hline 22 & 6 & 0 & -3.735040 & -1.899131 & 0.763950 \\
\hline 23 & 6 & 0 & -5.702305 & 1.127429 & 0.060943 \\
\hline 24 & 6 & 0 & -5.322537 & 2.159003 & 0.939086 \\
\hline 25 & 6 & 0 & -6.830527 & 1.334176 & -0.757595 \\
\hline 26 & 6 & 0 & -6.024087 & 3.359474 & 0.977597 \\
\hline 27 & 1 & 0 & -4.497508 & 2.003788 & 1.640793 \\
\hline 28 & 6 & 0 & -7.525554 & 2.536133 & -0.718311 \\
\hline 29 & 1 & 0 & -7.172710 & 0.533484 & -1.420449 \\
\hline 30 & 6 & 0 & -7.122174 & 3.557482 & 0.142480 \\
\hline 31 & 1 & 0 & -5.719291 & 4.142856 & 1.680481 \\
\hline 32 & 1 & 0 & -8.399253 & 2.675525 & -1.364302 \\
\hline 33 & 1 & 0 & -7.674900 & 4.502953 & 0.173118 \\
\hline 34 & 6 & 0 & -5.673264 & -1.433301 & -0.210751 \\
\hline 35 & 8 & 0 & -6.748687 & -1.657344 & -0.674998 \\
\hline 36 & 8 & 0 & -4.842308 & -2.454638 & 0.213000 \\
\hline 37 & 6 & 0 & -2.693031 & -2.604157 & 1.334256 \\
\hline 38 & 6 & 0 & -2.662759 & 0.223681 & 1.220153 \\
\hline 39 & 6 & 0 & -1.638806 & -1.861570 & 1.868416 \\
\hline 40 & 1 & 0 & -0.798558 & -2.394863 & 2.333201 \\
\hline 41 & 6 & 0 & -1.609651 & -0.454410 & 1.831180 \\
\hline 42 & 1 & 0 & -2.620753 & 1.317075 & 1.151803 \\
\hline 43 & 1 & 0 & -2.704114 & -3.698009 & 1.362289 \\
\hline 44 & 6 & 0 & -0.478441 & 0.301830 & 2.462427 \\
\hline 45 & 1 & 0 & -0.705565 & 0.563086 & 3.515805 \\
\hline 46 & 1 & 0 & -0.268467 & 1.250404 & 1.930953 \\
\hline 47 & 1 & 0 & 0.450109 & -0.300116 & 2.471501 \\
\hline 48 & 6 & 0 & 4.226311 & 0.671220 & 0.932731 \\
\hline 49 & 6 & 0 & 3.726033 & -0.596495 & 1.615887 \\
\hline 50 & 1 & 0 & 3.125983 & -0.349638 & 2.514752 \\
\hline 51 & 1 & 0 & 4.571282 & -1.235003 & 1.941643 \\
\hline 52 & 1 & 0 & 4.869322 & 0.413041 & 0.066507 \\
\hline 53 & 1 & 0 & 4.847645 & 1.291251 & 1.608417 \\
\hline 54 & 6 & 0 & 3.668236 & 2.803783 & -0.947087 \\
\hline 55 & 6 & 0 & 4.502878 & 3.832027 & -0.488923 \\
\hline 56 & 6 & 0 & 3.499830 & 2.605467 & -2.321649 \\
\hline 57 & 6 & 0 & 5.166038 & 4.646849 & -1.401764 \\
\hline 58 & 1 & 0 & 4.631454 & 4.000767 & 0.588955 \\
\hline 59 & 6 & 0 & 4.166738 & 3.423420 & -3.232658 \\
\hline 60 & 1 & 0 & 2.833884 & 1.806707 & -2.677720 \\
\hline 61 & 6 & 0 & 4.998938 & 4.441748 & -2.772345 \\
\hline 62 & 1 & 0 & 5.818415 & 5.450479 & -1.042331 \\
\hline 63 & 1 & 0 & 4.031996 & 3.267075 & -4.308703 \\
\hline 64 & 1 & 0 & 5.521343 & 5.086482 & -3.487986 \\
\hline 65 & 6 & 0 & 2.230081 & 2.746145 & 1.564509 \\
\hline 66 & 6 & 0 & 2.593969 & 2.584296 & 2.904977 \\
\hline 67 & 6 & 0 & 1.274581 & 3.717440 & 1.228945 \\
\hline 68 & 6 & 0 & 2.006466 & 3.375000 & 3.893857 \\
\hline 69 & 1 & 0 & 3.345613 & 1.840993 & 3.197981 \\
\hline 70 & 6 & 0 & 0.698332 & 4.511401 & 2.214206 \\
\hline 71 & 1 & 0 & 0.988637 & 3.855438 & 0.175880 \\
\hline 72 & 6 & 0 & 1.058438 & 4.335548 & 3.552107 \\
\hline 73 & 1 & 0 & 2.300338 & 3.241264 & 4.940969 \\
\hline 74 & 1 & 0 & -0.036011 & 5.277302 & 1.939795 \\
\hline 75 & 1 & 0 & 0.601831 & 4.957328 & 4.330059 \\
\hline 76 & 6 & 0 & 1.937487 & -2.925836 & 1.400684 \\
\hline 77 & 6 & 0 & 1.107947 & -3.811799 & 0.695187 \\
\hline 78 & 6 & 0 & 2.146174 & -3.120981 & 2.771624 \\
\hline 79 & 6 & 0 & 0.519514 & -4.889556 & 1.348426 \\
\hline 80 & 1 & 0 & 0.918645 & -3.642822 & -0.373889 \\
\hline 81 & 6 & 0 & 1.540277 & -4.194797 & 3.424820 \\
\hline 82 & 1 & 0 & 2.792477 & -2.445159 & 3.344891 \\
\hline 83 & 6 & 0 & 0.731381 & -5.080768 & 2.715562 \\
\hline
\end{tabular}




\begin{tabular}{|c|c|c|c|c|c|}
\hline 84 & 1 & 0 & -0.114399 & -5.586204 & 0.787764 \\
\hline 85 & 1 & 0 & 1.712606 & -4.345521 & 4.496422 \\
\hline 86 & 1 & 0 & 0.265263 & -5.928319 & 3.230402 \\
\hline 87 & 6 & 0 & 3.783820 & -2.288941 & -0.731913 \\
\hline 88 & 6 & 0 & 4.652884 & -3.313887 & -0.333459 \\
\hline 89 & 6 & 0 & 3.823077 & -1.820709 & -2.049872 \\
\hline 90 & 6 & 0 & 5.556328 & -3.854020 & -1.243645 \\
\hline 91 & 1 & 0 & 4.617980 & -3.699424 & 0.694786 \\
\hline 92 & 6 & 0 & 4.731313 & -2.362789 & -2.958397 \\
\hline 93 & 1 & 0 & 3.125076 & -1.031746 & -2.364873 \\
\hline 94 & 6 & 0 & 5.596799 & -3.377175 & -2.554972 \\
\hline 95 & 1 & 0 & 6.233035 & -4.656620 & -0.929780 \\
\hline 96 & 1 & 0 & 4.757354 & -1.995342 & -3.990240 \\
\hline 97 & 1 & 0 & 6.307478 & -3.806518 & -3.270051 \\
\hline
\end{tabular}

Cartesian coordinates (in Å) and energy of TS-Pd-C(5)-B $\mathrm{E}(\mathrm{UM} 06)=-2961.15244409 \mathrm{au}$

\begin{tabular}{|c|c|c|c|c|c|}
\hline \multirow{2}{*}{$\begin{array}{l}\text { Center } \\
\text { number }\end{array}$} & \multirow{2}{*}{$\begin{array}{l}\text { Atomic } \\
\text { number }\end{array}$} & \multirow{2}{*}{$\begin{array}{l}\text { Atomic } \\
\text { type }\end{array}$} & \multicolumn{3}{|c|}{ Coordinates (Angstroms) } \\
\hline & & & $X$ & $\mathrm{Y}$ & $\mathrm{Z}$ \\
\hline 1 & 46 & 0 & -1.327185 & -0.013501 & -0.442581 \\
\hline 2 & 15 & 0 & -2.436952 & 1.873374 & 0.474955 \\
\hline 3 & 15 & 0 & -3.073575 & -1.281589 & 0.459944 \\
\hline 4 & 8 & 0 & 0.289210 & 1.054922 & -1.606098 \\
\hline 5 & 8 & 0 & 0.174971 & -1.604227 & -1.772551 \\
\hline 6 & 6 & 0 & 1.383211 & -1.016446 & -1.934723 \\
\hline 7 & 6 & 0 & 1.351712 & 0.450931 & -1.857102 \\
\hline 8 & 6 & 0 & 2.547482 & -1.686130 & -2.151811 \\
\hline 9 & 6 & 0 & 2.595019 & 1.134444 & -2.104199 \\
\hline 10 & 6 & 0 & 3.768744 & 0.462611 & -2.317156 \\
\hline 11 & 1 & 0 & 2.547149 & 2.229947 & -2.142108 \\
\hline 12 & 1 & 0 & 0.268725 & -2.570036 & -1.690068 \\
\hline 13 & 1 & 0 & 2.562123 & -2.782844 & -2.185352 \\
\hline 14 & 6 & 0 & 3.775973 & -0.969734 & -2.186278 \\
\hline 15 & 1 & 0 & 4.673714 & -1.503599 & -2.527148 \\
\hline 16 & 6 & 0 & 5.028856 & 1.165390 & -2.702046 \\
\hline 17 & 1 & 0 & 5.924879 & 0.674078 & -2.281062 \\
\hline 18 & 1 & 0 & 5.025410 & 2.224043 & -2.386905 \\
\hline 19 & 1 & 0 & 5.146497 & 1.148575 & -3.804259 \\
\hline 20 & 6 & 0 & 4.331717 & -1.066867 & 0.061705 \\
\hline 21 & 6 & 0 & 5.597457 & -0.351210 & 0.147554 \\
\hline 22 & 6 & 0 & 6.590021 & -1.280271 & -0.198392 \\
\hline 23 & 6 & 0 & 3.076525 & -0.724344 & 0.693838 \\
\hline 24 & 6 & 0 & 2.721386 & 0.626039 & 0.921139 \\
\hline 25 & 6 & 0 & 2.143232 & -1.718546 & 1.076921 \\
\hline 26 & 6 & 0 & 1.515474 & 0.967626 & 1.521092 \\
\hline 27 & 1 & 0 & 3.400594 & 1.426457 & 0.621215 \\
\hline 28 & 6 & 0 & 0.943011 & -1.377400 & 1.681294 \\
\hline 29 & 1 & 0 & 2.391572 & -2.771032 & 0.914874 \\
\hline 30 & 6 & 0 & 0.619315 & -0.032293 & 1.900053 \\
\hline 31 & 1 & 0 & 1.266803 & 2.022703 & 1.689654 \\
\hline 32 & 1 & 0 & 0.249335 & -2.165878 & 2.000776 \\
\hline 33 & 1 & 0 & -0.313334 & 0.231869 & 2.414414 \\
\hline 34 & 6 & 0 & 4.728477 & -2.493834 & -0.206878 \\
\hline 35 & 8 & 0 & 4.066809 & -3.488441 & -0.276231 \\
\hline 36 & 8 & 0 & 6.077332 & -2.521617 & -0.440916 \\
\hline 37 & 6 & 0 & 7.934282 & -0.968515 & -0.263809 \\
\hline 38 & 6 & 0 & 5.999615 & 0.948163 & 0.489667 \\
\hline 39 & 6 & 0 & 8.297356 & 0.337968 & 0.059397 \\
\hline 40 & 1 & 0 & 9.356278 & 0.623025 & 0.022350 \\
\hline 41 & 6 & 0 & 7.350425 & 1.300331 & 0.451656 \\
\hline 42 & 1 & 0 & 5.275466 & 1.710085 & 0.795396 \\
\hline 43 & 1 & 0 & 8.670532 & -1.727971 & -0.543349 \\
\hline 44 & 6 & 0 & 7.801051 & 2.677416 & 0.838452 \\
\hline 45 & 1 & 0 & 8.513960 & 3.092446 & 0.102502 \\
\hline 46 & 1 & 0 & 8.319679 & 2.670442 & 1.816419 \\
\hline 47 & 1 & 0 & 6.952487 & 3.378767 & 0.921312 \\
\hline 48 & 6 & 0 & -4.267530 & -0.069950 & 1.215217 \\
\hline 49 & 6 & 0 & -3.562561 & 1.164283 & 1.767508 \\
\hline
\end{tabular}




\begin{tabular}{|c|c|c|c|c|c|}
\hline 50 & 1 & 0 & -2.932594 & 0.902269 & 2.641451 \\
\hline 51 & 1 & 0 & -4.294852 & 1.925751 & 2.102568 \\
\hline 52 & 1 & 0 & -4.948399 & 0.216968 & 0.388062 \\
\hline 53 & 1 & 0 & -4.894561 & -0.565328 & 1.982042 \\
\hline 54 & 6 & 0 & -4.088292 & -2.260515 & -0.694640 \\
\hline 55 & 6 & 0 & -5.141292 & -3.063442 & -0.235163 \\
\hline 56 & 6 & 0 & -3.821119 & -2.181905 & -2.065710 \\
\hline 57 & 6 & 0 & -5.921495 & -3.772514 & -1.143277 \\
\hline 58 & 1 & 0 & -5.348251 & -3.141158 & 0.840916 \\
\hline 59 & 6 & 0 & -4.604398 & -2.895871 & -2.972284 \\
\hline 60 & 1 & 0 & -2.986831 & -1.559410 & -2.421411 \\
\hline 61 & 6 & 0 & -5.653415 & -3.687944 & -2.510872 \\
\hline 62 & 1 & 0 & -6.744780 & -4.399333 & -0.782874 \\
\hline 63 & 1 & 0 & -4.391916 & -2.834466 & -4.045400 \\
\hline 64 & 1 & 0 & -6.268508 & -4.249806 & -3.222716 \\
\hline 65 & 6 & 0 & -2.616959 & -2.451001 & 1.782046 \\
\hline 66 & 6 & 0 & -2.734399 & -2.144589 & 3.142390 \\
\hline 67 & 6 & 0 & -2.018878 & -3.662936 & 1.404023 \\
\hline 68 & 6 & 0 & -2.262693 & -3.035348 & 4.107365 \\
\hline 69 & 1 & 0 & -3.204405 & -1.208129 & 3.468409 \\
\hline 70 & 6 & 0 & -1.558781 & -4.553593 & 2.368086 \\
\hline 71 & 1 & 0 & -1.921891 & -3.914138 & 0.337901 \\
\hline 72 & 6 & 0 & -1.675285 & -4.238467 & 3.723571 \\
\hline 73 & 1 & 0 & -2.363501 & -2.787615 & 5.169936 \\
\hline 74 & 1 & 0 & -1.105914 & -5.503353 & 2.062027 \\
\hline 75 & 1 & 0 & -1.309821 & -4.938070 & 4.483222 \\
\hline 76 & 6 & 0 & -1.488010 & 3.196420 & 1.279139 \\
\hline 77 & 6 & 0 & -0.583570 & 3.896980 & 0.465044 \\
\hline 78 & 6 & 0 & -1.558303 & 3.492385 & 2.645614 \\
\hline 79 & 6 & 0 & 0.221756 & 4.891073 & 1.010207 \\
\hline 80 & 1 & 0 & -0.506455 & 3.645196 & -0.602005 \\
\hline 81 & 6 & 0 & -0.738477 & 4.481853 & 3.188953 \\
\hline 82 & 1 & 0 & -2.259852 & 2.962785 & 3.301691 \\
\hline 83 & 6 & 0 & 0.148257 & 5.182465 & 2.374195 \\
\hline 84 & 1 & 0 & 0.916880 & 5.442795 & 0.367237 \\
\hline 85 & 1 & 0 & -0.801674 & 4.712287 & 4.258344 \\
\hline 86 & 1 & 0 & 0.785580 & 5.963411 & 2.803822 \\
\hline 87 & 6 & 0 & -3.564928 & 2.714391 & -0.686487 \\
\hline 88 & 6 & 0 & -4.273367 & 3.860563 & -0.300230 \\
\hline 89 & 6 & 0 & -3.737855 & 2.191751 & -1.972897 \\
\hline 90 & 6 & 0 & -5.153381 & 4.467302 & -1.191054 \\
\hline 91 & 1 & 0 & -4.129645 & 4.287351 & 0.702016 \\
\hline 92 & 6 & 0 & -4.622185 & 2.801734 & -2.861685 \\
\hline 93 & 1 & 0 & -3.163099 & 1.306140 & -2.280065 \\
\hline 94 & 6 & 0 & -5.329128 & 3.936546 & -2.470294 \\
\hline 95 & 1 & 0 & -5.705102 & 5.364115 & -0.887780 \\
\hline 96 & 1 & 0 & -4.753719 & 2.391527 & -3.869031 \\
\hline 97 & 1 & 0 & -6.021035 & 4.418352 & -3.170249 \\
\hline
\end{tabular}

Cartesian coordinates (in $\AA$ ) and energy of TS-Pd-C(5)-C $\mathrm{E}(\mathrm{UM} 06)=-2961.14372907 \mathrm{au}$

\begin{tabular}{rrrrrr}
$\begin{array}{c}\text { Center } \\
\text { number }\end{array}$ & $\begin{array}{c}\text { Atomic } \\
\text { number }\end{array}$ & $\begin{array}{c}\text { Atomic } \\
\text { type }\end{array}$ & \multicolumn{3}{c}{ Coordinates (Angstroms) } \\
\hline 1 & 46 & 0 & 1.667777 & -0.032848 & -0.764886 \\
2 & 15 & 0 & 2.738205 & -1.650543 & 0.504067 \\
3 & 15 & 0 & 2.933567 & 1.516689 & 0.436543 \\
4 & 8 & 0 & 0.468422 & -1.517166 & -1.763140 \\
5 & 8 & 0 & -0.198005 & 1.049692 & -2.035548 \\
6 & 6 & 0 & -1.205000 & 0.132848 & -2.007032 \\
7 & 6 & 0 & -0.768597 & -1.238375 & -1.784217 \\
8 & 6 & 0 & -2.518942 & 0.429793 & -2.182244 \\
9 & 6 & 0 & -1.785827 & -2.231769 & -1.651051 \\
10 & 6 & 0 & -3.123827 & -1.936578 & -1.750757 \\
11 & 1 & 0 & -1.443612 & -3.263434 & -1.504308 \\
12 & 1 & 0 & -0.548402 & 1.953618 & -2.123447 \\
13 & 1 & 0 & -2.826763 & 1.456997 & -2.421047 \\
14 & 6 & 0 & -3.521411 & -0.561601 & -1.918992 \\
15 & 1 & 0 & -4.524663 & -0.377667 & -2.323167
\end{tabular}




\begin{tabular}{|c|c|c|c|c|c|}
\hline 16 & 6 & 0 & -4.159150 & -3.006524 & -1.634648 \\
\hline 17 & 1 & 0 & -5.062671 & -2.777979 & -2.228342 \\
\hline 18 & 1 & 0 & -4.487524 & -3.116822 & -0.581202 \\
\hline 19 & 1 & 0 & -3.760917 & -3.984136 & -1.958163 \\
\hline 20 & 6 & 0 & -4.332266 & 0.072013 & 0.033019 \\
\hline 21 & 6 & 0 & -4.252331 & 1.521022 & -0.117209 \\
\hline 22 & 6 & 0 & -2.996378 & 1.897564 & 0.375533 \\
\hline 23 & 6 & 0 & -5.581488 & -0.688544 & 0.165630 \\
\hline 24 & 6 & 0 & -6.650321 & -0.465090 & -0.721004 \\
\hline 25 & 6 & 0 & -5.751285 & -1.646528 & 1.180656 \\
\hline 26 & 6 & 0 & -7.836444 & -1.180666 & -0.608225 \\
\hline 27 & 1 & 0 & -6.546117 & 0.260867 & -1.536316 \\
\hline 28 & 6 & 0 & -6.943825 & -2.354544 & 1.296621 \\
\hline 29 & 1 & 0 & -4.936947 & -1.837862 & 1.885845 \\
\hline 30 & 6 & 0 & -7.988270 & -2.130974 & 0.402055 \\
\hline 31 & 1 & 0 & -8.650951 & -0.997314 & -1.317802 \\
\hline 32 & 1 & 0 & -7.057083 & -3.092409 & 2.098740 \\
\hline 33 & 1 & 0 & -8.923275 & -2.694797 & 0.491987 \\
\hline 34 & 6 & 0 & -3.112304 & -0.291518 & 0.796320 \\
\hline 35 & 8 & 0 & -2.740019 & -1.346361 & 1.229887 \\
\hline 36 & 8 & 0 & -2.318878 & 0.833018 & 0.908789 \\
\hline 37 & 6 & 0 & -2.511148 & 3.187660 & 0.296697 \\
\hline 38 & 6 & 0 & -5.073878 & 2.498694 & -0.679973 \\
\hline 39 & 6 & 0 & -3.344689 & 4.143700 & -0.294291 \\
\hline 40 & 1 & 0 & -2.995479 & 5.180894 & -0.372716 \\
\hline 41 & 6 & 0 & -4.625606 & 3.823908 & -0.769886 \\
\hline 42 & 1 & 0 & -6.074374 & 2.249491 & -1.052653 \\
\hline 43 & 1 & 0 & -1.522980 & 3.447879 & 0.693482 \\
\hline 44 & 6 & 0 & -5.515647 & 4.890112 & -1.335455 \\
\hline 45 & 1 & 0 & -4.932430 & 5.709653 & -1.791469 \\
\hline 46 & 1 & 0 & -6.148042 & 5.342765 & -0.547017 \\
\hline 47 & 1 & 0 & -6.198579 & 4.487619 & -2.104571 \\
\hline 48 & 6 & 0 & 4.276940 & 0.530259 & 1.2602 \\
\hline 49 & 6 & 0 & 3.685552 & -0.758044 & 1.8146 \\
\hline 50 & 1 & 0 & 2.948453 & -0.542048 & 2.614530 \\
\hline 51 & 1 & 0 & 4.452325 & -1.418713 & 2.263465 \\
\hline 52 & 1 & 0 & 5.051349 & 0.322923 & 0.495379 \\
\hline 53 & 1 & 0 & 4.756029 & 1.136351 & 2.052800 \\
\hline 54 & 6 & 0 & 3.782680 & 2.899335 & -0.380196 \\
\hline 55 & 6 & 0 & 3.477993 & 4.239466 & -0.111839 \\
\hline 56 & 6 & 0 & 4.719008 & 2.590465 & -1.379221 \\
\hline 57 & 6 & 0 & 4.125432 & 5.254799 & -0.813690 \\
\hline 58 & 1 & 0 & 2.733794 & 4.496231 & 0.6520 \\
\hline 59 & 6 & 0 & 5.366527 & 3.608084 & -2.071228 \\
\hline 60 & 1 & 0 & 4.936947 & 1.542573 & -1.629874 \\
\hline 61 & 6 & 0 & 5.071920 & 4.942321 & -1.787288 \\
\hline 62 & 1 & 0 & 3.888008 & 6.301774 & -0.594476 \\
\hline 63 & 1 & 0 & 6.101432 & 3.358892 & -2.844539 \\
\hline 64 & 1 & 0 & 5.579942 & 5.743655 & -2.335072 \\
\hline 65 & 6 & 0 & 1.972393 & 2.171424 & 1.8389 \\
\hline 66 & 6 & 0 & 2.575265 & 2.915847 & 2.864063 \\
\hline 67 & 6 & 0 & 0.613016 & 1.848701 & 1.930361 \\
\hline 68 & 6 & 0 & 1.823431 & 3.332321 & 3.958815 \\
\hline 69 & 1 & 0 & 3.639897 & 3.180307 & 2.807928 \\
\hline 70 & 6 & 0 & -0.135467 & 2.260887 & 3.032607 \\
\hline 71 & 1 & 0 & 0.127501 & 1.262729 & 1.135195 \\
\hline 72 & 6 & 0 & 0.469357 & 3.002523 & 4.044877 \\
\hline 73 & 1 & 0 & 2.298637 & 3.915340 & 4.755548 \\
\hline 74 & 1 & 0 & -1.195571 & 1.988336 & 3.092919 \\
\hline 75 & 1 & 0 & -0.116144 & 3.325001 & 4.913174 \\
\hline 76 & 6 & 0 & 1.658720 & -2.847389 & 1.326005 \\
\hline 77 & 6 & 0 & 0.328043 & -2.500569 & 1.589298 \\
\hline 78 & 6 & 0 & 2.148173 & -4.104369 & 1.708197 \\
\hline 79 & 6 & 0 & -0.507208 & -3.404503 & 2.241450 \\
\hline 80 & 1 & 0 & -0.076971 & -1.527851 & 1.273300 \\
\hline 81 & 6 & 0 & 1.309195 & -4.999699 & 2.364728 \\
\hline 82 & 1 & 0 & 3.186075 & -4.386771 & 1.488002 \\
\hline 83 & 6 & 0 & -0.015411 & -4.649736 & 2.630280 \\
\hline 84 & 1 & 0 & -1.548988 & -3.122737 & 2.424086 \\
\hline 85 & 1 & 0 & 1.690761 & -5.981461 & 2.666602 \\
\hline 86 & 1 & 0 & -0.673844 & -5.362240 & 3.140163 \\
\hline 87 & 6 & 0 & 3.914066 & -2.589534 & -0.504057 \\
\hline
\end{tabular}




$\begin{array}{lllllr}88 & 6 & 0 & 5.282448 & -2.673756 & -0.220811 \\ 89 & 6 & 0 & 3.390492 & -3.247824 & -1.628388 \\ 90 & 6 & 0 & 6.119087 & -3.418173 & -1.051191 \\ 91 & 1 & 0 & 5.711669 & -2.169927 & 0.654043 \\ 92 & 6 & 0 & 4.231671 & -3.993010 & -2.447684 \\ 93 & 1 & 0 & 2.318790 & -3.164549 & -1.857189 \\ 94 & 6 & 0 & 5.595140 & -4.077942 & -2.161010 \\ 95 & 1 & 0 & 7.188790 & -3.486435 & -0.824253 \\ 96 & 1 & 0 & 3.820826 & -4.509085 & -3.322331 \\ 97 & 1 & 0 & 6.255652 & -4.663226 & -2.810370\end{array}$

Cartesian coordinates (in Å) and energy of TS-Pd-C(5)-D $\mathrm{E}(\mathrm{UM} 06)=-2961.14710612 \mathrm{au}$

\begin{tabular}{|c|c|c|c|c|c|}
\hline \multirow{2}{*}{$\begin{array}{l}\text { Center } \\
\text { number }\end{array}$} & \multirow{2}{*}{$\begin{array}{l}\text { Atomic } \\
\text { number }\end{array}$} & \multirow{2}{*}{$\begin{array}{l}\text { Atomic } \\
\text { type }\end{array}$} & \multicolumn{3}{|c|}{ Coordinates (Angstroms) } \\
\hline & & & $\mathrm{X}$ & $\mathrm{Y}$ & $\mathrm{Z}$ \\
\hline 1 & 8 & 0 & 1.823701 & 0.146850 & 0.631845 \\
\hline 2 & 8 & 0 & -0.008255 & 1.237229 & -2.096717 \\
\hline 3 & 8 & 0 & -0.416730 & -1.387481 & -1.871458 \\
\hline 4 & 6 & 0 & 3.003834 & -0.091130 & 0.549989 \\
\hline 5 & 6 & 0 & 4.089411 & 0.657952 & -0.123284 \\
\hline 6 & 6 & 0 & 5.295381 & -0.096112 & 0.178415 \\
\hline 7 & 6 & 0 & 6.663114 & 0.070689 & -0.074840 \\
\hline 8 & 1 & 0 & 7.040383 & 0.955355 & -0.599410 \\
\hline 9 & 6 & 0 & 7.575542 & -0.894372 & 0.360120 \\
\hline 10 & 6 & 0 & 7.100822 & -2.023511 & 1.052180 \\
\hline 11 & 1 & 0 & 7.823527 & -2.777841 & 1.387810 \\
\hline 12 & 6 & 0 & 5.747731 & -2.206905 & 1.335081 \\
\hline 13 & 1 & 0 & 5.382003 & -3.078774 & 1.885946 \\
\hline 14 & 6 & 0 & 4.878896 & -1.231863 & 0.886336 \\
\hline 15 & 6 & 0 & 3.439010 & -0.035694 & -2.149585 \\
\hline 16 & 6 & 0 & 2.346223 & 0.856232 & -2.353984 \\
\hline 17 & 1 & 0 & 2.542631 & 1.917568 & -2.556552 \\
\hline 18 & 6 & 0 & 1.075261 & 0.426465 & -2.131918 \\
\hline 19 & 6 & 0 & 0.774846 & -0.992313 & -1.937285 \\
\hline 20 & 6 & 0 & 1.893940 & -1.887641 & -1.883130 \\
\hline 21 & 6 & 0 & 3.185112 & -1.453749 & -2.033687 \\
\hline 22 & 6 & 0 & 4.018576 & 2.109773 & -0.334384 \\
\hline 23 & 6 & 0 & 2.979493 & 2.878965 & 0.218425 \\
\hline 24 & 1 & 0 & 2.226790 & 2.397741 & 0.849265 \\
\hline 25 & 6 & 0 & 2.894977 & 4.245693 & -0.029065 \\
\hline 26 & 1 & 0 & 2.082822 & 4.825680 & 0.425270 \\
\hline 27 & 6 & 0 & 3.837243 & 4.876417 & -0.840468 \\
\hline 28 & 1 & 0 & 3.767900 & 5.951907 & -1.037503 \\
\hline 29 & 6 & 0 & 4.873783 & 4.126338 & -1.396229 \\
\hline 30 & 1 & 0 & 5.619092 & 4.609673 & -2.037624 \\
\hline 31 & 6 & 0 & 4.964933 & 2.761481 & -1.143582 \\
\hline 32 & 1 & 0 & 5.769134 & 2.188301 & -1.618468 \\
\hline 33 & 6 & 0 & 9.046895 & -0.726850 & 0.124437 \\
\hline 34 & 1 & 0 & 9.508443 & -1.662868 & -0.239686 \\
\hline 35 & 1 & 0 & 9.573851 & -0.452427 & 1.058584 \\
\hline 36 & 1 & 0 & 9.256160 & 0.065045 & -0.615529 \\
\hline 37 & 1 & 0 & 1.661231 & -2.952792 & -1.763513 \\
\hline 38 & 6 & 0 & 4.332769 & -2.406928 & -2.117368 \\
\hline 39 & 1 & 0 & 5.252802 & -1.994343 & -1.663160 \\
\hline 40 & 1 & 0 & 4.099831 & -3.370782 & -1.631133 \\
\hline 41 & 1 & 0 & 4.574049 & -2.620047 & -3.178347 \\
\hline 42 & 8 & 0 & 3.523767 & -1.245287 & 1.072912 \\
\hline 43 & 1 & 0 & 4.429986 & 0.264386 & -2.515822 \\
\hline 44 & 46 & 0 & -1.773359 & -0.034925 & -0.802806 \\
\hline 45 & 15 & 0 & -2.670736 & -1.743764 & 0.507309 \\
\hline 46 & 15 & 0 & -3.079022 & 1.421203 & 0.474311 \\
\hline 47 & 6 & 0 & -3.605823 & -0.914506 & 1.869783 \\
\hline 48 & 6 & 0 & -4.305715 & 0.337961 & 1.359623 \\
\hline 49 & 1 & 0 & -2.842016 & -0.655215 & 2.630966 \\
\hline 50 & 1 & 0 & -4.306270 & -1.620625 & 2.356137 \\
\hline 51 & 1 & 0 & -4.780726 & 0.904428 & 2.183575 \\
\hline 52 & 1 & 0 & -5.105346 & 0.085249 & 0.635201 \\
\hline 53 & 6 & 0 & -3.829305 & -2.784353 & -0.420915 \\
\hline
\end{tabular}




\begin{tabular}{lllrrr}
54 & 6 & 0 & -5.167997 & -2.973830 & -0.059424 \\
55 & 6 & 0 & -3.323496 & -3.406974 & -1.573218 \\
56 & 6 & 0 & -5.990833 & -3.786470 & -0.838504 \\
57 & 1 & 0 & -5.583826 & -2.497752 & 0.837213 \\
58 & 6 & 0 & -4.148448 & -4.222080 & -2.340688 \\
59 & 1 & 0 & -2.276409 & -3.242729 & -1.864893 \\
60 & 6 & 0 & -5.482360 & -4.411341 & -1.975153 \\
61 & 1 & 0 & -7.037387 & -3.935324 & -0.550227 \\
62 & 1 & 0 & -3.749595 & -4.710136 & -3.236781 \\
63 & 1 & 0 & -6.131607 & -5.050333 & -2.584027 \\
64 & 6 & 0 & -1.468406 & -2.854081 & 1.286456 \\
65 & 6 & 0 & -0.175429 & -2.368234 & 1.515882 \\
66 & 6 & 0 & -1.806772 & -4.160400 & 1.662968 \\
67 & 6 & 0 & 0.772130 & -3.182174 & 2.131609 \\
68 & 1 & 0 & 0.107470 & -1.354211 & 1.199292 \\
69 & 6 & 0 & -0.854476 & -4.967685 & 2.279514 \\
70 & 1 & 0 & -2.814954 & -4.550301 & 1.471216 \\
71 & 6 & 0 & 0.431732 & -4.479368 & 2.513788 \\
72 & 1 & 0 & 1.783637 & -2.794005 & 2.296623 \\
73 & 1 & 0 & -1.117681 & -5.988895 & 2.577190 \\
74 & 1 & 0 & 1.177481 & -5.122614 & 2.994925 \\
75 & 6 & 0 & -2.077896 & 2.111323 & 1.835321 \\
76 & 6 & 0 & -0.699427 & 1.863732 & 1.847565 \\
77 & 6 & 0 & -2.663340 & 2.803904 & 2.905703 \\
78 & 6 & 0 & 0.081329 & 2.296720 & 2.918824 \\
79 & 1 & 0 & -0.223894 & 1.315989 & 1.020466 \\
80 & 6 & 0 & -1.877913 & 3.245554 & 3.966654 \\
81 & 1 & 0 & -3.742760 & 3.007511 & 2.911920 \\
82 & 6 & 0 & -0.505568 & 2.988951 & 3.975598 \\
83 & 1 & 0 & 1.155326 & 2.075447 & 2.927774 \\
84 & 1 & 0 & -2.340946 & 3.788705 & 4.798046 \\
85 & 1 & 0 & 0.107425 & 3.328042 & 4.818282 \\
86 & 6 & 0 & -4.067536 & 2.769624 & -0.240811 \\
87 & 6 & 0 & -5.067313 & 2.431060 & -1.165851 \\
88 & 6 & 0 & -3.805413 & 4.119677 & 0.023640 \\
89 & 6 & 0 & -5.816865 & 3.425713 & -1.784737 \\
90 & 1 & 0 & -5.255513 & 1.377977 & -1.417905 \\
91 & 6 & 0 & -4.554749 & 5.112452 & -0.605370 \\
92 & 1 & 0 & -3.013476 & 4.402146 & 0.728136 \\
93 & 6 & 0 & -5.563068 & 4.768721 & -1.503295 \\
94 & 1 & 0 & -6.600529 & 3.151480 & -2.499594 \\
95 & 1 & 0 & -4.348474 & 6.166508 & -0.388225 \\
96 & 1 & 0 & -6.151505 & 5.552003 & -1.993751 \\
97 & 1 & 0 & 0.252900 & 2.174386 & -2.136374 \\
\hline--------------------------------------------------------------------------- \\
\hline 5 & 6 & 0 & 0 & 0 & 0
\end{tabular}

Cartesian coordinates (in $\AA$ ) and energy of TS-Pd-C(5)-E $\mathrm{E}(\mathrm{UM} 06)=-2961.14843132 \mathrm{au}$

\begin{tabular}{cccccc}
$\begin{array}{c}\text { Center } \\
\text { number }\end{array}$ & $\begin{array}{c}\text { Atomic } \\
\text { number }\end{array}$ & $\begin{array}{c}\text { Atomic } \\
\text { type }\end{array}$ & \multicolumn{3}{c}{ Coordinates (Angstroms) } \\
\hline 1 & 8 & 0 & Y & $Z$ \\
2 & 8 & 0 & 0.745611 & 1.035007 & -1.739694 \\
3 & 1 & 0 & 1.150499 & 1.918047 & -1.686402 \\
4 & 8 & 0 & -0.048178 & -1.497636 & -1.528986 \\
5 & 6 & 0 & 5.223647 & -1.943165 & 0.288031 \\
6 & 6 & 0 & 4.440883 & -0.658690 & 0.241666 \\
7 & 6 & 0 & 5.458170 & 0.381903 & 0.113705 \\
8 & 6 & 0 & 5.464641 & 1.780362 & 0.006703 \\
9 & 1 & 0 & 4.538424 & 2.363832 & 0.053611 \\
10 & 6 & 0 & 6.665154 & 2.471195 & -0.179127 \\
11 & 6 & 0 & 7.862971 & 1.743697 & -0.281911 \\
12 & 1 & 0 & 8.802532 & 2.289767 & -0.433050 \\
13 & 6 & 0 & 7.889562 & 0.352799 & -0.206734 \\
14 & 1 & 0 & 8.817242 & -0.220187 & -0.296464 \\
15 & 6 & 0 & 6.682268 & -0.290364 & -0.013122 \\
16 & 6 & 0 & 3.934732 & -0.792799 & -2.014487 \\
17 & 6 & 0 & 3.020492 & 0.299898 & -2.029610 \\
18 & 1 & 0 & 3.409234 & 1.326293 & -2.090279 \\
19 & 6 & 0 & 1.692370 & 0.072813 & -1.843920
\end{tabular}




\begin{tabular}{|c|c|c|c|c|c|}
\hline 20 & 6 & 0 & 1.166018 & -1.296181 & -1.739417 \\
\hline 21 & 6 & 0 & 2.111023 & -2.364999 & -1.919283 \\
\hline 22 & 6 & 0 & 3.448116 & -2.138002 & -2.122730 \\
\hline 23 & 6 & 0 & 3.134287 & -0.571381 & 0.850849 \\
\hline 24 & 6 & 0 & 2.345625 & -1.728906 & 1.068306 \\
\hline 25 & 1 & 0 & 2.766100 & -2.710167 & 0.840450 \\
\hline 26 & 6 & 0 & 1.072329 & -1.635235 & 1.621992 \\
\hline 27 & 1 & 0 & 0.487051 & -2.547525 & 1.793635 \\
\hline 28 & 6 & 0 & 0.540825 & -0.391611 & 1.956640 \\
\hline 29 & 1 & 0 & -0.445071 & -0.323048 & 2.433307 \\
\hline 30 & 6 & 0 & 1.305595 & 0.768656 & 1.762200 \\
\hline 31 & 1 & 0 & 0.904858 & 1.745331 & 2.062274 \\
\hline 32 & 6 & 0 & 2.574900 & 0.676938 & 1.218431 \\
\hline 33 & 1 & 0 & 3.166676 & 1.589345 & 1.112405 \\
\hline 34 & 6 & 0 & 6.691017 & 3.968649 & -0.255774 \\
\hline 35 & 1 & 0 & 5.679399 & 4.391049 & -0.388767 \\
\hline 36 & 1 & 0 & 7.318702 & 4.321764 & -1.094372 \\
\hline 37 & 1 & 0 & 7.113269 & 4.411070 & 0.667089 \\
\hline 38 & 1 & 0 & 1.698664 & -3.381747 & -1.922642 \\
\hline 39 & 6 & 0 & 4.390910 & -3.248887 & -2.456059 \\
\hline 40 & 1 & 0 & 4.137744 & -4.176798 & -1.914354 \\
\hline 41 & 1 & 0 & 4.341550 & -3.469416 & -3.540840 \\
\hline 42 & 1 & 0 & 5.440499 & -2.994994 & -2.226156 \\
\hline 43 & 8 & 0 & 6.541552 & -1.645612 & 0.055836 \\
\hline 44 & 1 & 0 & 4.974164 & -0.597027 & -2.308367 \\
\hline 45 & 46 & 0 & -1.254376 & 0.053310 & -0.438565 \\
\hline 46 & 15 & 0 & -2.905116 & -1.376333 & 0.497483 \\
\hline 47 & 15 & 0 & -2.552790 & 1.819725 & 0.368595 \\
\hline 48 & 6 & 0 & -3.794683 & -0.311128 & 1.728028 \\
\hline 49 & 6 & 0 & -4.077500 & 1.055760 & 1.114173 \\
\hline 50 & 1 & 0 & -4.731560 & -0.802813 & 2.057884 \\
\hline 51 & 1 & 0 & -3.138644 & -0.217614 & 2.616742 \\
\hline 52 & 1 & 0 & -4.545781 & 1.747096 & 1.841808 \\
\hline 53 & 1 & 0 & -4.790075 & 0.955176 & 0.270123 \\
\hline 54 & 6 & 0 & -4.203398 & -1.887393 & -0.676841 \\
\hline 55 & 6 & 0 & -5.226895 & -2.759601 & -0.281219 \\
\hline 56 & 6 & 0 & -4.184130 & -1.385555 & -1.982852 \\
\hline 57 & 6 & 0 & -6.225591 & -3.113849 & -1.182941 \\
\hline 58 & 1 & 0 & -5.238261 & -3.172384 & 0.736973 \\
\hline 59 & 6 & 0 & -5.188115 & -1.741737 & -2.882538 \\
\hline 60 & 1 & 0 & -3.366271 & -0.720813 & -2.296355 \\
\hline 61 & 6 & 0 & -6.207064 & -2.603213 & -2.482126 \\
\hline 62 & 1 & 0 & -7.023844 & -3.797250 & -0.872541 \\
\hline 63 & 1 & 0 & -5.169153 & -1.349621 & -3.905377 \\
\hline 64 & 1 & 0 & -6.993509 & -2.886620 & -3.190627 \\
\hline 65 & 6 & 0 & -2.405839 & -2.887040 & 1.370413 \\
\hline 66 & 6 & 0 & -2.567577 & -3.086703 & 2.746653 \\
\hline 67 & 6 & 0 & -1.745658 & -3.859719 & 0.602392 \\
\hline 68 & 6 & 0 & -2.078110 & -4.247866 & 3.345447 \\
\hline 69 & 1 & 0 & -3.084545 & -2.344889 & 3.367576 \\
\hline 70 & 6 & 0 & -1.269908 & -5.019872 & 1.203496 \\
\hline 71 & 1 & 0 & -1.593431 & -3.691914 & -0.472910 \\
\hline 72 & 6 & 0 & -1.431519 & -5.213556 & 2.577184 \\
\hline 73 & 1 & 0 & -2.210890 & -4.400609 & 4.422316 \\
\hline 74 & 1 & 0 & -0.762270 & -5.778898 & 0.597789 \\
\hline 75 & 1 & 0 & -1.050810 & -6.125162 & 3.050927 \\
\hline 76 & 6 & 0 & -3.195188 & 3.016474 & -0.846694 \\
\hline 77 & 6 & 0 & -2.929563 & 2.811915 & -2.204976 \\
\hline 78 & 6 & 0 & -3.971331 & 4.112815 & -0.446191 \\
\hline 79 & 6 & 0 & -3.441229 & 3.692886 & -3.157474 \\
\hline 80 & 1 & 0 & -2.311101 & 1.956300 & -2.514111 \\
\hline 81 & 6 & 0 & -4.481355 & 4.988245 & -1.399889 \\
\hline 82 & 1 & 0 & -4.173081 & 4.287619 & 0.619445 \\
\hline 83 & 6 & 0 & -4.217116 & 4.777567 & -2.754591 \\
\hline 84 & 1 & 0 & -3.230934 & 3.531259 & -4.220562 \\
\hline 85 & 1 & 0 & -5.089115 & 5.844080 & -1.085630 \\
\hline 86 & 1 & 0 & -4.619252 & 5.470109 & -3.502543 \\
\hline 87 & 6 & 0 & -1.797924 & 2.847692 & 1.672367 \\
\hline 88 & 6 & 0 & -2.042558 & 2.651667 & 3.036110 \\
\hline 89 & 6 & 0 & -0.850207 & 3.805210 & 1.279785 \\
\hline 90 & 6 & 0 & -1.352081 & 3.400656 & 3.989984 \\
\hline 91 & 1 & 0 & -2.783451 & 1.915698 & 3.373165 \\
\hline
\end{tabular}




$\begin{array}{llllll}92 & 6 & 0 & -0.171908 & 4.558549 & 2.232034 \\ 93 & 1 & 0 & -0.652436 & 3.969034 & 0.210508 \\ 94 & 6 & 0 & -0.417867 & 4.353546 & 3.591396 \\ 95 & 1 & 0 & -1.554597 & 3.241813 & 5.055012 \\ 96 & 1 & 0 & 0.552930 & 5.316527 & 1.913813 \\ 97 & 1 & 0 & 0.117638 & 4.944862 & 4.342224\end{array}$

Cartesian coordinates (in Å) and energy of TS-Pd-C(5)-F $\mathrm{E}(\mathrm{UM} 06)=-2961.15432310 \mathrm{au}$

\begin{tabular}{|c|c|c|c|c|c|}
\hline \multirow{2}{*}{$\begin{array}{l}\text { Center } \\
\text { number }\end{array}$} & \multirow{2}{*}{$\begin{array}{l}\text { Atomic } \\
\text { number }\end{array}$} & \multirow{2}{*}{$\begin{array}{l}\text { Atomic } \\
\text { type }\end{array}$} & \multicolumn{3}{|c|}{ Coordinates (Angstroms) } \\
\hline & & & $\mathrm{X}$ & $\mathrm{Y}$ & $\mathrm{Z}$ \\
\hline 1 & 8 & 0 & 5.054126 & 3.008334 & 0.462706 \\
\hline 2 & 8 & 0 & 0.567090 & 1.663166 & -1.495443 \\
\hline 3 & 1 & 0 & 0.690240 & 2.619823 & -1.359283 \\
\hline 4 & 8 & 0 & 0.548553 & -1.006880 & -1.577581 \\
\hline 5 & 6 & 0 & 4.368098 & 2.053245 & 0.653285 \\
\hline 6 & 6 & 0 & 4.634295 & 0.592904 & 0.426604 \\
\hline 7 & 6 & 0 & 3.447354 & -0.071658 & 0.855667 \\
\hline 8 & 6 & 0 & 3.000048 & -1.408817 & 0.837422 \\
\hline 9 & 1 & 0 & 3.659200 & -2.211016 & 0.485131 \\
\hline 10 & 6 & 0 & 1.707984 & -1.722852 & 1.252874 \\
\hline 11 & 6 & 0 & 0.870395 & -0.683808 & 1.706016 \\
\hline 12 & 1 & 0 & -0.124717 & -0.943852 & 2.090315 \\
\hline 13 & 6 & 0 & 1.271362 & 0.656606 & 1.714701 \\
\hline 14 & 1 & 0 & 0.609398 & 1.451925 & 2.074747 \\
\hline 15 & 6 & 0 & 2.540648 & 0.933653 & 1.248879 \\
\hline 16 & 6 & 0 & 4.142387 & 0.872400 & -1.870673 \\
\hline 17 & 6 & 0 & 2.957195 & 1.651031 & -1.785007 \\
\hline 18 & 1 & 0 & 3.032273 & 2.743735 & -1.702872 \\
\hline 19 & 6 & 0 & 1.748163 & 1.029294 & -1.679116 \\
\hline 20 & 6 & 0 & 1.638299 & -0.428856 & -1.775899 \\
\hline 21 & 6 & 0 & 2.844222 & -1.140771 & -2.115282 \\
\hline 22 & 6 & 0 & 4.065628 & -0.523468 & -2.196237 \\
\hline 23 & 6 & 0 & 6.001164 & 0.079984 & 0.407053 \\
\hline 24 & 6 & 0 & 7.056298 & 0.835290 & -0.141853 \\
\hline 25 & 1 & 0 & 6.857199 & 1.834551 & -0.541674 \\
\hline 26 & 6 & 0 & 8.348348 & 0.326626 & -0.170878 \\
\hline 27 & 1 & 0 & 9.155006 & 0.925505 & -0.607624 \\
\hline 28 & 6 & 0 & 8.623228 & -0.935802 & 0.357760 \\
\hline 29 & 1 & 0 & 9.644200 & -1.332380 & 0.333075 \\
\hline 30 & 6 & 0 & 7.596306 & -1.680381 & 0.935806 \\
\hline 31 & 1 & 0 & 7.808699 & -2.658326 & 1.381563 \\
\hline 32 & 6 & 0 & 6.299722 & -1.178250 & 0.962661 \\
\hline 33 & 1 & 0 & 5.512897 & -1.756013 & 1.458283 \\
\hline 34 & 6 & 0 & 1.186002 & -3.122780 & 1.150944 \\
\hline 35 & 1 & 0 & 0.650132 & -3.257764 & 0.188491 \\
\hline 36 & 1 & 0 & 0.466438 & -3.355026 & 1.958141 \\
\hline 37 & 1 & 0 & 2.000138 & -3.868345 & 1.187844 \\
\hline 38 & 1 & 0 & 2.732890 & -2.216211 & -2.304558 \\
\hline 39 & 6 & 0 & 5.307505 & -1.274918 & -2.550448 \\
\hline 40 & 1 & 0 & 5.934132 & -1.468996 & -1.655473 \\
\hline 41 & 1 & 0 & 5.068675 & -2.249094 & -3.010844 \\
\hline 42 & 1 & 0 & 5.938428 & -0.701995 & -3.254935 \\
\hline 43 & 8 & 0 & 3.076421 & 2.178613 & 1.137968 \\
\hline 44 & 1 & 0 & 5.092645 & 1.395603 & -2.032851 \\
\hline 45 & 46 & 0 & -1.148564 & 0.071251 & -0.472987 \\
\hline 46 & 15 & 0 & -2.755345 & -1.618075 & 0.055776 \\
\hline 47 & 15 & 0 & -2.815340 & 1.612031 & 0.078375 \\
\hline 48 & 6 & 0 & -4.275972 & -0.703748 & 0.626374 \\
\hline 49 & 6 & 0 & -4.396834 & 0.657912 & -0.041683 \\
\hline 50 & 1 & 0 & -4.189595 & -0.600852 & 1.724770 \\
\hline 51 & 1 & 0 & -5.174914 & -1.321081 & 0.440915 \\
\hline 52 & 1 & 0 & -5.245187 & 1.247139 & 0.359052 \\
\hline 53 & 1 & 0 & -4.577120 & 0.545216 & -1.130695 \\
\hline 54 & 6 & 0 & -3.281020 & -2.584220 & -1.398097 \\
\hline 55 & 6 & 0 & -4.483014 & -3.306968 & -1.412340 \\
\hline 56 & 6 & 0 & -2.441259 & -2.616251 & -2.518154 \\
\hline 57 & 6 & 0 & -4.844731 & -4.039899 & -2.538169 \\
\hline 58 & 1 & 0 & -5.139273 & -3.316732 & -0.531827 \\
\hline
\end{tabular}




\begin{tabular}{|c|c|c|c|c|c|}
\hline 59 & 6 & 0 & -2.806990 & -3.356090 & -3.643003 \\
\hline 60 & 1 & 0 & -1.490690 & -2.065006 & -2.504254 \\
\hline 61 & 6 & 0 & -4.007600 & -4.061985 & -3.655022 \\
\hline 62 & 1 & 0 & -5.785368 & -4.601835 & -2.544293 \\
\hline 63 & 1 & 0 & -2.146998 & -3.378615 & -4.517238 \\
\hline 64 & 1 & 0 & -4.295203 & -4.639147 & -4.541041 \\
\hline 65 & 6 & 0 & -2.472609 & -2.827201 & 1.392650 \\
\hline 66 & 6 & 0 & -2.301699 & -2.354672 & 2.703854 \\
\hline 67 & 6 & 0 & -2.320084 & -4.194869 & 1.134483 \\
\hline 68 & 6 & 0 & -2.011402 & -3.237858 & 3.739592 \\
\hline 69 & 1 & 0 & -2.394124 & -1.280284 & 2.924274 \\
\hline 70 & 6 & 0 & -2.025972 & -5.075481 & 2.174440 \\
\hline 71 & 1 & 0 & -2.436678 & -4.579653 & 0.113623 \\
\hline 72 & 6 & 0 & -1.874195 & -4.601770 & 3.476259 \\
\hline 73 & 1 & 0 & -1.891095 & -2.859370 & 4.760895 \\
\hline 74 & 1 & 0 & -1.918429 & -6.145419 & 1.963645 \\
\hline 75 & 1 & 0 & -1.647480 & -5.297701 & 4.291525 \\
\hline 76 & 6 & 0 & -2.744103 & 2.311535 & 1.759511 \\
\hline 77 & 6 & 0 & -1.621171 & 3.091177 & 2.077354 \\
\hline 78 & 6 & 0 & -3.719011 & 2.092092 & 2.738836 \\
\hline 79 & 6 & 0 & -1.477899 & 3.643059 & 3.345724 \\
\hline 80 & 1 & 0 & -0.855487 & 3.277324 & 1.309087 \\
\hline 81 & 6 & 0 & -3.567957 & 2.637126 & 4.014983 \\
\hline 82 & 1 & 0 & -4.617931 & 1.504467 & 2.515562 \\
\hline 83 & 6 & 0 & -2.450843 & 3.410090 & 4.320080 \\
\hline 84 & 1 & 0 & -0.602245 & 4.259466 & 3.578210 \\
\hline 85 & 1 & 0 & -4.338161 & 2.461104 & 4.774198 \\
\hline 86 & 1 & 0 & -2.337403 & 3.839269 & 5.321636 \\
\hline 87 & 6 & 0 & -3.062684 & 3.040744 & -1.026972 \\
\hline 88 & 6 & 0 & -2.677981 & 2.906377 & -2.367978 \\
\hline 89 & 6 & 0 & -3.651896 & 4.233959 & -0.589605 \\
\hline 90 & 6 & 0 & -2.890806 & 3.951097 & -3.264352 \\
\hline 91 & 1 & 0 & -2.205363 & 1.973266 & -2.707640 \\
\hline 92 & 6 & 0 & -3.855394 & 5.278096 & -1.488879 \\
\hline 93 & 1 & 0 & -3.953501 & 4.350776 & 0.459457 \\
\hline 94 & 6 & 0 & -3.477019 & 5.137403 & -2.823969 \\
\hline 95 & 1 & 0 & -2.592706 & 3.840503 & -4.312946 \\
\hline 96 & 1 & 0 & -4.316286 & 6.210534 & -1.144215 \\
\hline 97 & 1 & 0 & -3.638939 & 5.961807 & -3.527411 \\
\hline
\end{tabular}

Cartesian coordinates (in $\AA$ ) and energy of INT-II-Pd-C(5)-A $\mathrm{E}(\mathrm{UM} 06)=-2961.17439447 \mathrm{au}$

\begin{tabular}{rrrrrr}
$\begin{array}{c}\text { Center } \\
\text { number }\end{array}$ & $\begin{array}{c}\text { Atomic } \\
\text { number }\end{array}$ & $\begin{array}{c}\text { Atomic } \\
\text { type }\end{array}$ & \multicolumn{3}{c}{ Coordinates (Angstroms) } \\
\hline 1 & 46 & 0 & 1.412515 & 0.161742 & -0.854167 \\
2 & 15 & 0 & 2.611870 & -1.532813 & 0.325708 \\
3 & 15 & 0 & 2.689739 & 1.699865 & 0.356188 \\
4 & 8 & 0 & 0.081572 & -1.248340 & -2.053150 \\
5 & 8 & 0 & -0.537338 & 1.330495 & -2.158000 \\
6 & 6 & 0 & -1.563384 & 0.443151 & -2.163781 \\
7 & 6 & 0 & -1.116931 & -0.961415 & -2.157236 \\
8 & 6 & 0 & -2.868679 & 0.750593 & -2.143497 \\
9 & 6 & 0 & -2.141709 & -1.972041 & -2.301811 \\
10 & 6 & 0 & -3.463958 & -1.692726 & -2.242318 \\
11 & 1 & 0 & -1.784251 & -2.997205 & -2.461426 \\
12 & 1 & 0 & -0.863842 & 2.247153 & -2.166962 \\
13 & 1 & 0 & -3.188008 & 1.802101 & -2.127336 \\
14 & 6 & 0 & -3.931260 & -0.283376 & -1.998374 \\
15 & 1 & 0 & -4.751981 & -0.075292 & -2.716332 \\
16 & 6 & 0 & -4.473066 & -2.779060 & -2.421333 \\
17 & 1 & 0 & -5.333914 & -2.441814 & -3.025120 \\
18 & 1 & 0 & -4.888676 & -3.125447 & -1.455139 \\
19 & 1 & 0 & -4.015860 & -3.658255 & -2.906233 \\
20 & 6 & 0 & -4.580724 & -0.090575 & -0.560729 \\
21 & 6 & 0 & -3.683161 & -0.599508 & 0.533394 \\
22 & 6 & -4.341508 & -1.656099 & 1.152941 \\
23 & 6 & -5.087272 & 1.341825 & -0.389517 \\
24 & 6 & -4.664660 & 2.150307 & 0.670060 \\
& 6 & 0 & & &
\end{tabular}




\begin{tabular}{|c|c|c|c|c|c|}
\hline 25 & 6 & 0 & -6.007384 & 1.866961 & -1.307997 \\
\hline 26 & 6 & 0 & -5.133692 & 3.458291 & 0.797286 \\
\hline 27 & 1 & 0 & -3.969983 & 1.755483 & 1.419646 \\
\hline 28 & 6 & 0 & -6.472412 & 3.172278 & -1.180681 \\
\hline 29 & 1 & 0 & -6.393567 & 1.241999 & -2.120868 \\
\hline 30 & 6 & 0 & -6.033196 & 3.976331 & -0.129598 \\
\hline 31 & 1 & 0 & -4.796412 & 4.073586 & 1.639472 \\
\hline 32 & 1 & 0 & -7.195198 & 3.561073 & -1.906535 \\
\hline 33 & 1 & 0 & -6.403110 & 5.002592 & -0.027845 \\
\hline 34 & 6 & 0 & -5.794132 & -1.035705 & -0.422099 \\
\hline 35 & 8 & 0 & -6.772950 & -1.065717 & -1.097313 \\
\hline 36 & 8 & 0 & -5.583595 & -1.895810 & 0.613295 \\
\hline 37 & 6 & 0 & -3.787340 & -2.387811 & 2.187166 \\
\hline 38 & 6 & 0 & -2.413195 & -0.243398 & 0.978496 \\
\hline 39 & 6 & 0 & -2.510839 & -2.017500 & 2.613467 \\
\hline 40 & 1 & 0 & -2.038855 & -2.573725 & 3.433349 \\
\hline 41 & 6 & 0 & -1.813756 & -0.949294 & 2.032450 \\
\hline 42 & 1 & 0 & -1.874148 & 0.602996 & 0.530276 \\
\hline 43 & 1 & 0 & -4.338265 & -3.214244 & 2.646235 \\
\hline 44 & 6 & 0 & -0.470530 & -0.529815 & 2.549648 \\
\hline 45 & 1 & 0 & -0.558607 & 0.300889 & 3.279604 \\
\hline 46 & 1 & 0 & 0.178667 & -0.164765 & 1.725585 \\
\hline 47 & 1 & 0 & 0.049116 & -1.365107 & 3.055181 \\
\hline 48 & 6 & 0 & 4.076690 & 0.701715 & 1.079030 \\
\hline 49 & 6 & 0 & 3.584407 & -0.631948 & 1.626314 \\
\hline 50 & 1 & 0 & 2.910563 & -0.481252 & 2.494185 \\
\hline 51 & 1 & 0 & 4.434801 & -1.254418 & 1.967443 \\
\hline 52 & 1 & 0 & 4.783744 & 0.542394 & 0.238545 \\
\hline 53 & 1 & 0 & 4.629639 & 1.287668 & 1.838476 \\
\hline 54 & 6 & 0 & 3.531060 & 3.102221 & -0.444928 \\
\hline 55 & 6 & 0 & 3.651095 & 4.357298 & 0.163014 \\
\hline 56 & 6 & 0 & 4.084475 & 2.888844 & -1.715191 \\
\hline 57 & 6 & 0 & 4.329183 & 5.383300 & -0.492762 \\
\hline 58 & 1 & 0 & 3.214514 & 4.538416 & 1.153433 \\
\hline 59 & 6 & 0 & 4.768467 & 3.914178 & -2.361050 \\
\hline 60 & 1 & 0 & 3.972087 & 1.909479 & -2.203326 \\
\hline 61 & 6 & 0 & 4.889226 & 5.162760 & -1.749882 \\
\hline 62 & 1 & 0 & 4.422311 & 6.364878 & -0.014822 \\
\hline 63 & 1 & 0 & 5.202897 & 3.741984 & -3.352009 \\
\hline 64 & 1 & 0 & 5.421276 & 5.972608 & -2.261344 \\
\hline 65 & 6 & 0 & 1.763794 & 2.399457 & 1.764105 \\
\hline 66 & 6 & 0 & 2.251726 & 2.435117 & 3.074741 \\
\hline 67 & 6 & 0 & 0.485764 & 2.910601 & 1.497180 \\
\hline 68 & 6 & 0 & 1.470724 & 2.968347 & 4.100420 \\
\hline 69 & 1 & 0 & 3.251032 & 2.049851 & 3.312223 \\
\hline 70 & 6 & 0 & -0.288267 & 3.452277 & 2.518453 \\
\hline 71 & 1 & 0 & 0.095387 & 2.877312 & 0.469828 \\
\hline 72 & 6 & 0 & 0.202906 & 3.476637 & 3.824673 \\
\hline 73 & 1 & 0 & 1.860636 & 2.990887 & 5.124117 \\
\hline 74 & 1 & 0 & -1.283083 & 3.855630 & 2.296620 \\
\hline 75 & 1 & 0 & -0.406235 & 3.896922 & 4.632608 \\
\hline 76 & 6 & 0 & 1.720625 & -2.858824 & 1.197918 \\
\hline 77 & 6 & 0 & 0.577991 & -3.374044 & 0.570448 \\
\hline 78 & 6 & 0 & 2.098025 & -3.345141 & 2.456148 \\
\hline 79 & 6 & 0 & -0.173609 & -4.367016 & 1.193062 \\
\hline 80 & 1 & 0 & 0.276195 & -2.982339 & -0.411279 \\
\hline 81 & 6 & 0 & 1.340868 & -4.338265 & 3.075676 \\
\hline 82 & 1 & 0 & 2.987495 & -2.953083 & 2.965771 \\
\hline 83 & 6 & 0 & 0.206417 & -4.849228 & 2.445314 \\
\hline 84 & 1 & 0 & -1.069196 & -4.762099 & 0.699496 \\
\hline 85 & 1 & 0 & 1.642479 & -4.719064 & 4.057965 \\
\hline 86 & 1 & 0 & -0.385389 & -5.630607 & 2.935581 \\
\hline 87 & 6 & 0 & 3.878309 & -2.340226 & -0.711356 \\
\hline 88 & 6 & 0 & 4.639543 & -3.410920 & -0.224231 \\
\hline 89 & 6 & 0 & 4.097454 & -1.869523 & -2.011194 \\
\hline 90 & 6 & 0 & 5.616518 & -3.993069 & -1.026122 \\
\hline 91 & 1 & 0 & 4.462147 & -3.802719 & 0.786547 \\
\hline 92 & 6 & 0 & 5.079038 & -2.453648 & -2.810732 \\
\hline 93 & 1 & 0 & 3.479984 & -1.047724 & -2.403358 \\
\hline 94 & 6 & 0 & 5.837712 & -3.513103 & -2.317923 \\
\hline 95 & 1 & 0 & 6.207856 & -4.832107 & -0.642869 \\
\hline 96 & 1 & 0 & 5.245168 & -2.084719 & -3.828919 \\
\hline
\end{tabular}


Cartesian coordinates (in ̊̊) and energy of INT-II-Pd-C(5)-B $\mathrm{E}(\mathrm{UM} 06)=-2961.16784202 \mathrm{au}$

\begin{tabular}{|c|c|c|c|c|c|}
\hline \multirow{2}{*}{$\begin{array}{l}\text { Center } \\
\text { number }\end{array}$} & \multirow{2}{*}{$\begin{array}{l}\text { Atomic } \\
\text { number }\end{array}$} & \multirow{2}{*}{$\begin{array}{l}\text { Atomic } \\
\text { type }\end{array}$} & \multicolumn{3}{|c|}{ Coordinates (Angstroms) } \\
\hline & & & $X$ & $\mathrm{Y}$ & $\mathrm{Z}$ \\
\hline 1 & 46 & 0 & -1.340894 & 0.012306 & -0.547434 \\
\hline 2 & 15 & 0 & -2.362278 & 1.858138 & 0.534642 \\
\hline 3 & 15 & 0 & -2.997284 & -1.299546 & 0.461768 \\
\hline 4 & 8 & 0 & 0.280031 & 1.074144 & -1.816474 \\
\hline 5 & 8 & 0 & 0.062353 & -1.586244 & -1.938539 \\
\hline 6 & 6 & 0 & 1.302929 & -1.045800 & -2.064164 \\
\hline 7 & 6 & 0 & 1.300875 & 0.433846 & -2.078177 \\
\hline 8 & 6 & 0 & 2.451290 & -1.735431 & -2.113532 \\
\hline 9 & 6 & 0 & 2.546176 & 1.068027 & -2.483892 \\
\hline 10 & 6 & 0 & 3.707971 & 0.379714 & -2.539265 \\
\hline 11 & 1 & 0 & 2.480369 & 2.120422 & -2.787875 \\
\hline 12 & 1 & 0 & 0.124464 & -2.537421 & -1.740944 \\
\hline 13 & 1 & 0 & 2.457337 & -2.828956 & -2.024288 \\
\hline 14 & 6 & 0 & 3.756694 & -1.023311 & -2.012290 \\
\hline 15 & 1 & 0 & 4.546800 & -1.579556 & -2.554508 \\
\hline 16 & 6 & 0 & 4.937864 & 0.933125 & -3.172636 \\
\hline 17 & 1 & 0 & 5.862472 & 0.637993 & -2.645843 \\
\hline 18 & 1 & 0 & 4.904747 & 2.033316 & -3.260653 \\
\hline 19 & 1 & 0 & 5.018135 & 0.518902 & -4.199155 \\
\hline 20 & 6 & 0 & 4.212682 & -1.056815 & -0.449989 \\
\hline 21 & 6 & 0 & 5.487449 & -0.304534 & -0.133202 \\
\hline 22 & 6 & 0 & 6.426411 & -1.239676 & 0.288121 \\
\hline 23 & 6 & 0 & 3.043670 & -0.695823 & 0.468649 \\
\hline 24 & 6 & 0 & 2.750735 & 0.645304 & 0.748736 \\
\hline 25 & 6 & 0 & 2.176171 & -1.674581 & 0.980564 \\
\hline 26 & 6 & 0 & 1.620018 & 1.004088 & 1.481662 \\
\hline 27 & 1 & 0 & 3.408882 & 1.440697 & 0.391269 \\
\hline 28 & 6 & 0 & 1.052277 & -1.322182 & 1.722702 \\
\hline 29 & 1 & 0 & 2.371551 & -2.734947 & 0.791646 \\
\hline 30 & 6 & 0 & 0.760052 & 0.021217 & 1.963214 \\
\hline 31 & 1 & 0 & 1.412673 & 2.063636 & 1.675744 \\
\hline 32 & 1 & 0 & 0.398860 & -2.108366 & 2.121101 \\
\hline 33 & 1 & 0 & -0.120742 & 0.297762 & 2.556682 \\
\hline 34 & 6 & 0 & 4.678396 & -2.513469 & -0.217356 \\
\hline 35 & 8 & 0 & 4.073806 & -3.520853 & -0.434009 \\
\hline 36 & 8 & 0 & 5.947809 & -2.526793 & 0.244477 \\
\hline 37 & 6 & 0 & 7.705153 & -0.912130 & 0.700128 \\
\hline 38 & 6 & 0 & 5.859173 & 1.039995 & -0.140118 \\
\hline 39 & 6 & 0 & 8.048046 & 0.437239 & 0.682798 \\
\hline 40 & 1 & 0 & 9.055151 & 0.738531 & 0.996823 \\
\hline 41 & 6 & 0 & 7.142458 & 1.424987 & 0.267809 \\
\hline 42 & 1 & 0 & 5.170360 & 1.818850 & -0.490131 \\
\hline 43 & 1 & 0 & 8.404833 & -1.690464 & 1.018710 \\
\hline 44 & 6 & 0 & 7.543139 & 2.870283 & 0.279814 \\
\hline 45 & 1 & 0 & 8.576260 & 3.008495 & -0.087209 \\
\hline 46 & 1 & 0 & 7.508976 & 3.289165 & 1.304312 \\
\hline 47 & 1 & 0 & 6.875355 & 3.486553 & -0.347941 \\
\hline 48 & 6 & 0 & -4.138744 & -0.115519 & 1.328527 \\
\hline 49 & 6 & 0 & -3.393441 & 1.096039 & 1.876599 \\
\hline 50 & 1 & 0 & -2.706535 & 0.798950 & 2.694085 \\
\hline 51 & 1 & 0 & -4.097797 & 1.845986 & 2.288587 \\
\hline 52 & 1 & 0 & -4.869632 & 0.199900 & 0.556228 \\
\hline 53 & 1 & 0 & -4.717399 & -0.635943 & 2.116561 \\
\hline 54 & 6 & 0 & -4.088520 & -2.277943 & -0.621050 \\
\hline 55 & 6 & 0 & -5.084744 & -3.107342 & -0.087830 \\
\hline 56 & 6 & 0 & -3.939223 & -2.173693 & -2.008266 \\
\hline 57 & 6 & 0 & -5.927258 & -3.815948 & -0.938668 \\
\hline 58 & 1 & 0 & -5.198028 & -3.205935 & 1.000404 \\
\hline 59 & 6 & 0 & -4.784268 & -2.887865 & -2.857358 \\
\hline 60 & 1 & 0 & -3.148711 & -1.531041 & -2.423074 \\
\hline 61 & 6 & 0 & -5.777374 & -3.705384 & -2.322414 \\
\hline 62 & 1 & 0 & -6.706401 & -4.463104 & -0.520701 \\
\hline
\end{tabular}




\begin{tabular}{|c|c|c|c|c|c|}
\hline 63 & 1 & 0 & -4.664025 & -2.806603 & -3.943352 \\
\hline 64 & 1 & 0 & -6.441057 & -4.267210 & -2.989201 \\
\hline 65 & 6 & 0 & -2.422491 & -2.480651 & 1.726380 \\
\hline 66 & 6 & 0 & -2.496703 & -2.228916 & 3.100609 \\
\hline 67 & 6 & 0 & -1.792697 & -3.652317 & 1.279839 \\
\hline 68 & 6 & 0 & -1.951865 & -3.135014 & 4.011855 \\
\hline 69 & 1 & 0 & -2.990202 & -1.325823 & 3.481010 \\
\hline 70 & 6 & 0 & -1.258776 & -4.558262 & 2.189753 \\
\hline 71 & 1 & 0 & -1.732581 & -3.861198 & 0.201769 \\
\hline 72 & 6 & 0 & -1.333394 & -4.298107 & 3.559940 \\
\hline 73 & 1 & 0 & -2.019504 & -2.930765 & 5.086223 \\
\hline 74 & 1 & 0 & -0.781282 & -5.476733 & 1.830326 \\
\hline 75 & 1 & 0 & -0.910409 & -5.009564 & 4.277557 \\
\hline 76 & 6 & 0 & -1.376471 & 3.165033 & 1.323632 \\
\hline 77 & 6 & 0 & -0.556354 & 3.927666 & 0.477567 \\
\hline 78 & 6 & 0 & -1.350939 & 3.401085 & 2.703248 \\
\hline 79 & 6 & 0 & 0.262236 & 4.920620 & 1.004653 \\
\hline 80 & 1 & 0 & -0.560675 & 3.733408 & -0.604190 \\
\hline 81 & 6 & 0 & -0.519057 & 4.390689 & 3.227875 \\
\hline 82 & 1 & 0 & -1.985751 & 2.823256 & 3.385901 \\
\hline 83 & 6 & 0 & 0.285002 & 5.151199 & 2.381827 \\
\hline 84 & 1 & 0 & 0.891243 & 5.519727 & 0.336638 \\
\hline 85 & 1 & 0 & -0.507452 & 4.573132 & 4.308250 \\
\hline 86 & 1 & 0 & 0.932553 & 5.931413 & 2.797164 \\
\hline 87 & 6 & 0 & -3.586630 & 2.736197 & -0.496818 \\
\hline 88 & 6 & 0 & -4.229852 & 3.888453 & -0.024372 \\
\hline 89 & 6 & 0 & -3.908546 & 2.232110 & -1.761952 \\
\hline 90 & 6 & 0 & -5.190463 & 4.520459 & -0.808072 \\
\hline 91 & 1 & 0 & -3.973041 & 4.300105 & 0.961385 \\
\hline 92 & 6 & 0 & -4.874427 & 2.866134 & -2.542458 \\
\hline 93 & 1 & 0 & -3.387412 & 1.340070 & -2.138814 \\
\hline 94 & 6 & 0 & -5.514172 & 4.008134 & -2.065522 \\
\hline 95 & 1 & 0 & -5.690014 & 5.422333 & -0.437000 \\
\hline 96 & 1 & 0 & -5.122884 & 2.469179 & -3.532934 \\
\hline 97 & 1 & 0 & -6.269678 & 4.509294 & -2.680988 \\
\hline
\end{tabular}

Cartesian coordinates (in Å) and energy of INT-II-Pd-C(5)-C $\mathrm{E}(\mathrm{UM} 06)=-2961.16986503 \mathrm{au}$

\begin{tabular}{|c|c|c|c|c|c|}
\hline \multirow{2}{*}{$\begin{array}{l}\text { Center } \\
\text { number }\end{array}$} & \multirow{2}{*}{$\begin{array}{l}\text { Atomic } \\
\text { number }\end{array}$} & \multirow{2}{*}{$\begin{array}{l}\text { Atomic } \\
\text { type }\end{array}$} & \multicolumn{3}{|c|}{ Coordinates (Angstroms) } \\
\hline & & & $\mathrm{X}$ & $\mathrm{Y}$ & $\mathrm{Z}$ \\
\hline 1 & 46 & 0 & 1.842564 & 0.040725 & -0.899469 \\
\hline 2 & 15 & 0 & 2.981526 & -1.525597 & 0.460326 \\
\hline 3 & 15 & 0 & 2.882230 & 1.704816 & 0.375249 \\
\hline 4 & 8 & 0 & 0.544678 & -1.569567 & -1.899809 \\
\hline 5 & 8 & 0 & -0.167940 & 0.953817 & -2.281508 \\
\hline 6 & 6 & 0 & -1.157632 & 0.057814 & -2.064862 \\
\hline 7 & 6 & 0 & -0.665780 & -1.326404 & -1.900219 \\
\hline 8 & 6 & 0 & -2.469909 & 0.334425 & -2.017963 \\
\hline 9 & 6 & 0 & -1.670092 & -2.357882 & -1.774146 \\
\hline 10 & 6 & 0 & -2.993369 & -2.103905 & -1.646821 \\
\hline 11 & 1 & 0 & -1.290092 & -3.386356 & -1.756486 \\
\hline 12 & 1 & 0 & -0.516936 & 1.859944 & -2.345922 \\
\hline 13 & 1 & 0 & -2.813048 & 1.366648 & -2.175398 \\
\hline 14 & 6 & 0 & -3.507511 & -0.685931 & -1.671262 \\
\hline 15 & 1 & 0 & -4.308268 & -0.640614 & -2.441865 \\
\hline 16 & 6 & 0 & -3.950656 & -3.239970 & -1.508812 \\
\hline 17 & 1 & 0 & -4.833840 & -3.117771 & -2.162162 \\
\hline 18 & 1 & 0 & -4.337546 & -3.318068 & -0.474174 \\
\hline 19 & 1 & 0 & -3.456365 & -4.194666 & -1.756759 \\
\hline 20 & 6 & 0 & -4.216540 & -0.226961 & -0.326864 \\
\hline 21 & 6 & 0 & -4.586359 & 1.238988 & -0.358690 \\
\hline 22 & 6 & 0 & -3.793941 & 1.894751 & 0.573951 \\
\hline 23 & 6 & 0 & -5.422643 & -1.067921 & 0.065317 \\
\hline 24 & 6 & 0 & -6.469595 & -1.237488 & -0.849299 \\
\hline 25 & 6 & 0 & -5.549802 & -1.633979 & 1.337139 \\
\hline 26 & 6 & 0 & -7.598574 & -1.977778 & -0.514490 \\
\hline 27 & 1 & 0 & -6.405177 & -0.801313 & -1.854698 \\
\hline 28 & 6 & 0 & -6.683451 & -2.373169 & 1.675199 \\
\hline
\end{tabular}




\begin{tabular}{|c|c|c|c|c|c|}
\hline 29 & 1 & 0 & -4.753826 & -1.520552 & 2.081339 \\
\hline 30 & 6 & 0 & -7.707439 & -2.554335 & 0.750707 \\
\hline 31 & 1 & 0 & -8.401231 & -2.106104 & -1.249184 \\
\hline 32 & 1 & 0 & -6.760898 & -2.813543 & 2.675429 \\
\hline 33 & 1 & 0 & -8.594234 & -3.140317 & 1.015566 \\
\hline 34 & 6 & 0 & -3.118616 & -0.226491 & 0.749939 \\
\hline 35 & 8 & 0 & -2.462035 & -1.147944 & 1.130612 \\
\hline 36 & 8 & 0 & -2.929860 & 1.034836 & 1.219663 \\
\hline 37 & 6 & 0 & -3.857176 & 3.256897 & 0.795178 \\
\hline 38 & 6 & 0 & -5.496498 & 1.969936 & -1.110020 \\
\hline 39 & 6 & 0 & -4.776532 & 3.975958 & 0.028830 \\
\hline 40 & 1 & 0 & -4.858496 & 5.060288 & 0.173712 \\
\hline 41 & 6 & 0 & -5.603179 & 3.356685 & -0.919873 \\
\hline 42 & 1 & 0 & -6.139547 & 1.480474 & -1.853140 \\
\hline 43 & 1 & 0 & -3.211103 & 3.741306 & 1.534549 \\
\hline 44 & 6 & 0 & -6.608308 & 4.155540 & -1.693898 \\
\hline 45 & 1 & 0 & -6.275873 & 5.198432 & -1.840004 \\
\hline 46 & 1 & 0 & -7.579380 & 4.197109 & -1.163086 \\
\hline 47 & 1 & 0 & -6.802875 & 3.715849 & -2.688308 \\
\hline 48 & 6 & 0 & 4.248168 & 0.812360 & 1.274130 \\
\hline 49 & 6 & 0 & 3.732432 & -0.514737 & 1.816377 \\
\hline 50 & 1 & 0 & 2.914448 & -0.348071 & 2.546981 \\
\hline 51 & 1 & 0 & 4.518187 & -1.081770 & 2.351503 \\
\hline 52 & 1 & 0 & 5.071048 & 0.655093 & 0.548353 \\
\hline 53 & 1 & 0 & 4.647371 & 1.454737 & 2.082418 \\
\hline 54 & 6 & 0 & 3.692517 & 3.161089 & -0.359532 \\
\hline 55 & 6 & 0 & 3.274679 & 4.473040 & -0.104906 \\
\hline 56 & 6 & 0 & 4.715837 & 2.935689 & -1.293171 \\
\hline 57 & 6 & 0 & 3.892668 & 5.541778 & -0.752392 \\
\hline 58 & 1 & 0 & 2.462618 & 4.667240 & 0.606399 \\
\hline 59 & 6 & 0 & 5.334502 & 4.006042 & -1.930541 \\
\hline 60 & 1 & 0 & 5.026237 & 1.909871 & -1.536995 \\
\hline 61 & 6 & 0 & 4.925028 & 5.312094 & -1.659216 \\
\hline 62 & 1 & 0 & 3.563699 & 6.565765 & -0.542380 \\
\hline 63 & 1 & 0 & 6.137909 & 3.819447 & -2.651787 \\
\hline 64 & 1 & 0 & 5.410195 & 6.154846 & -2.163986 \\
\hline 65 & 6 & 0 & 1.825609 & 2.291349 & 1.746338 \\
\hline 66 & 6 & 0 & 2.349145 & 3.068108 & 2.790937 \\
\hline 67 & 6 & 0 & 0.484534 & 1.891120 & 1.797497 \\
\hline 68 & 6 & 0 & 1.542742 & 3.439001 & 3.862733 \\
\hline 69 & 1 & 0 & 3.397420 & 3.395072 & 2.767818 \\
\hline 70 & 6 & 0 & -0.319622 & 2.254655 & 2.877845 \\
\hline 71 & 1 & 0 & 0.066180 & 1.277174 & 0.984100 \\
\hline 72 & 6 & 0 & 0.209463 & 3.028761 & 3.908100 \\
\hline 73 & 1 & 0 & 1.959387 & 4.047682 & 4.673059 \\
\hline 74 & 1 & 0 & -1.361359 & 1.917782 & 2.908998 \\
\hline 75 & 1 & 0 & -0.419872 & 3.312569 & 4.759246 \\
\hline 76 & 6 & 0 & 1.947150 & -2.783296 & 1.266830 \\
\hline 77 & 6 & 0 & 0.601473 & -2.475362 & 1.505910 \\
\hline 78 & 6 & 0 & 2.452881 & -4.034124 & 1.642966 \\
\hline 79 & 6 & 0 & -0.228664 & -3.404543 & 2.127268 \\
\hline 80 & 1 & 0 & 0.187827 & -1.504044 & 1.196962 \\
\hline 81 & 6 & 0 & 1.618174 & -4.960380 & 2.263475 \\
\hline 82 & 1 & 0 & 3.502651 & -4.288196 & 1.446935 \\
\hline 83 & 6 & 0 & 0.280671 & -4.646539 & 2.505741 \\
\hline 84 & 1 & 0 & -1.279675 & -3.148824 & 2.299573 \\
\hline 85 & 1 & 0 & 2.015697 & -5.937917 & 2.558876 \\
\hline 86 & 1 & 0 & -0.372029 & -5.381792 & 2.990194 \\
\hline 87 & 6 & 0 & 4.328019 & -2.421718 & -0.366313 \\
\hline 88 & 6 & 0 & 5.661733 & -2.396603 & 0.056071 \\
\hline 89 & 6 & 0 & 3.978327 & -3.153453 & -1.512114 \\
\hline 90 & 6 & 0 & 6.632554 & -3.102286 & -0.654420 \\
\hline 91 & 1 & 0 & 5.960152 & -1.831491 & 0.947699 \\
\hline 92 & 6 & 0 & 4.948857 & -3.863446 & -2.210564 \\
\hline 93 & 1 & 0 & 2.933091 & -3.161765 & -1.852939 \\
\hline 94 & 6 & 0 & 6.277716 & -3.836614 & -1.783691 \\
\hline 95 & 1 & 0 & 7.675017 & -3.080465 & -0.317572 \\
\hline 96 & 1 & 0 & 4.668814 & -4.437844 & -3.100557 \\
\hline 97 & 1 & 0 & 7.042750 & -4.391223 & -2.338408 \\
\hline
\end{tabular}


Cartesian coordinates (in $\AA$ ) and energy of INT-II-Pd-C(5)-D

$\mathrm{E}(\mathrm{UM} 06)=-2961.17178634 \mathrm{au}$

\begin{tabular}{|c|c|c|c|c|c|}
\hline \multirow{2}{*}{$\begin{array}{l}\text { Center } \\
\text { number }\end{array}$} & \multirow{2}{*}{$\begin{array}{l}\text { Atomic } \\
\text { number }\end{array}$} & \multirow{2}{*}{$\begin{array}{l}\text { Atomic } \\
\text { type }\end{array}$} & \multicolumn{3}{|c|}{ Coordinates (Angstroms) } \\
\hline & & & $X$ & $\mathrm{Y}$ & Z \\
\hline 1 & 8 & 0 & 1.992202 & 0.297214 & 0.857717 \\
\hline 2 & 8 & 0 & -0.124304 & 1.136743 & -2.285625 \\
\hline 3 & 8 & 0 & -0.477801 & -1.469612 & -1.915657 \\
\hline 4 & 6 & 0 & 3.130247 & -0.024934 & 0.682674 \\
\hline 5 & 6 & 0 & 4.081900 & 0.412541 & -0.444897 \\
\hline 6 & 6 & 0 & 5.334785 & -0.336391 & -0.073677 \\
\hline 7 & 6 & 0 & 6.600856 & -0.386052 & -0.639312 \\
\hline 8 & 1 & 0 & 6.838415 & 0.199776 & -1.537982 \\
\hline 9 & 6 & 0 & 7.586338 & -1.198158 & -0.059908 \\
\hline 10 & 6 & 0 & 7.264124 & -1.936996 & 1.089351 \\
\hline 11 & 1 & 0 & 8.034991 & -2.575050 & 1.539059 \\
\hline 12 & 6 & 0 & 6.001964 & -1.880876 & 1.683572 \\
\hline 13 & 1 & 0 & 5.757615 & -2.452790 & 2.583755 \\
\hline 14 & 6 & 0 & 5.066263 & -1.066609 & 1.074834 \\
\hline 15 & 6 & 0 & 3.418118 & -0.050309 & -1.810151 \\
\hline 16 & 6 & 0 & 2.244789 & 0.826961 & -2.094418 \\
\hline 17 & 1 & 0 & 2.437069 & 1.902945 & -2.201568 \\
\hline 18 & 6 & 0 & 0.982815 & 0.374900 & -2.122070 \\
\hline 19 & 6 & 0 & 0.685145 & -1.058690 & -1.952150 \\
\hline 20 & 6 & 0 & 1.825582 & -1.952866 & -1.882267 \\
\hline 21 & 6 & 0 & 3.108767 & -1.527324 & -1.848368 \\
\hline 22 & 6 & 0 & 4.357935 & 1.911299 & -0.408712 \\
\hline 23 & 6 & 0 & 4.121113 & 2.657977 & 0.749572 \\
\hline 24 & 1 & 0 & 3.673229 & 2.186396 & 1.632946 \\
\hline 25 & 6 & 0 & 4.460439 & 4.008796 & 0.809699 \\
\hline 26 & 1 & 0 & 4.266821 & 4.573836 & 1.728284 \\
\hline 27 & 6 & 0 & 5.044126 & 4.636104 & -0.287187 \\
\hline 28 & 1 & 0 & 5.309552 & 5.697856 & -0.240689 \\
\hline 29 & 6 & 0 & 5.294308 & 3.899575 & -1.443697 \\
\hline 30 & 1 & 0 & 5.760042 & 4.378832 & -2.312038 \\
\hline 31 & 6 & 0 & 4.958220 & 2.549418 & -1.501431 \\
\hline 32 & 1 & 0 & 5.172690 & 1.993871 & -2.423578 \\
\hline 33 & 6 & 0 & 8.970445 & -1.252631 & -0.633211 \\
\hline 34 & 1 & 0 & 9.418850 & -2.255693 & -0.519905 \\
\hline 35 & 1 & 0 & 9.643784 & -0.538835 & -0.119931 \\
\hline 36 & 1 & 0 & 8.979993 & -0.993080 & -1.706485 \\
\hline 37 & 1 & 0 & 1.585017 & -3.023054 & -1.896414 \\
\hline 38 & 6 & 0 & 4.227335 & -2.519110 & -1.898594 \\
\hline 39 & 1 & 0 & 4.682016 & -2.700155 & -0.905860 \\
\hline 40 & 1 & 0 & 3.864571 & -3.490409 & -2.276658 \\
\hline 41 & 1 & 0 & 5.046359 & -2.170237 & -2.553989 \\
\hline 42 & 8 & 0 & 3.761838 & -0.904399 & 1.500328 \\
\hline 43 & 1 & 0 & 4.200437 & 0.131674 & -2.578544 \\
\hline 44 & 46 & 0 & -1.996445 & -0.039609 & -0.941706 \\
\hline 45 & 15 & 0 & -2.795526 & -1.694346 & 0.544532 \\
\hline 46 & 15 & 0 & -3.229677 & 1.497986 & 0.319778 \\
\hline 47 & 6 & 0 & -3.624462 & -0.751678 & 1.905220 \\
\hline 48 & 6 & 0 & -4.376193 & 0.450735 & 1.348686 \\
\hline 49 & 1 & 0 & -2.804629 & -0.423687 & 2.576533 \\
\hline 50 & 1 & 0 & -4.277712 & -1.409901 & 2.509263 \\
\hline 51 & 1 & 0 & -4.818879 & 1.063483 & 2.157501 \\
\hline 52 & 1 & 0 & -5.208969 & 0.130518 & 0.690862 \\
\hline 53 & 6 & 0 & -3.998954 & -2.868470 & -0.138769 \\
\hline 54 & 6 & 0 & -5.287894 & -3.056336 & 0.371873 \\
\hline 55 & 6 & 0 & -3.591841 & -3.587568 & -1.273797 \\
\hline 56 & 6 & 0 & -6.155805 & -3.960690 & -0.239771 \\
\hline 57 & 1 & 0 & -5.629917 & -2.503075 & 1.255241 \\
\hline 58 & 6 & 0 & -4.457358 & -4.496551 & -1.872284 \\
\hline 59 & 1 & 0 & -2.585178 & -3.425791 & -1.685625 \\
\hline 60 & 6 & 0 & -5.741567 & -4.682123 & -1.357098 \\
\hline 61 & 1 & 0 & -7.163772 & -4.104564 & 0.165150 \\
\hline 62 & 1 & 0 & -4.132078 & -5.059621 & -2.754114 \\
\hline 63 & 1 & 0 & -6.425735 & -5.392842 & -1.833945 \\
\hline 64 & 6 & 0 & -1.484414 & -2.677233 & 1.329645 \\
\hline 65 & 6 & 0 & -0.261014 & -2.035202 & 1.566604 \\
\hline 66 & 6 & 0 & -1.643832 & -4.022866 & 1.679935 \\
\hline
\end{tabular}




\begin{tabular}{|c|c|c|c|c|c|}
\hline 67 & 6 & 0 & 0.788797 & -2.726486 & 2.163786 \\
\hline 68 & 1 & 0 & -0.118995 & -0.984167 & 1.274927 \\
\hline 69 & 6 & 0 & -0.587856 & -4.712446 & 2.273020 \\
\hline 70 & 1 & 0 & -2.594943 & -4.535959 & 1.488341 \\
\hline 71 & 6 & 0 & 0.624454 & -4.066815 & 2.515679 \\
\hline 72 & 1 & 0 & 1.739315 & -2.210540 & 2.344302 \\
\hline 73 & 1 & 0 & -0.714548 & -5.765300 & 2.549504 \\
\hline 74 & 1 & 0 & 1.450102 & -4.616579 & 2.982023 \\
\hline 75 & 6 & 0 & -2.168172 & 2.290181 & 1.579004 \\
\hline 76 & 6 & 0 & -0.784341 & 2.083764 & 1.520966 \\
\hline 77 & 6 & 0 & -2.707164 & 3.029953 & 2.642334 \\
\hline 78 & 6 & 0 & 0.048426 & 2.605026 & 2.510092 \\
\hline 79 & 1 & 0 & -0.344943 & 1.497586 & 0.699809 \\
\hline 80 & 6 & 0 & -1.872554 & 3.556907 & 3.623590 \\
\hline 81 & 1 & 0 & -3.789827 & 3.204365 & 2.704571 \\
\hline 82 & 6 & 0 & -0.494418 & 3.342465 & 3.559274 \\
\hline 83 & 1 & 0 & 1.126722 & 2.418437 & 2.454466 \\
\hline 84 & 1 & 0 & -2.300847 & 4.135832 & 4.449526 \\
\hline 85 & 1 & 0 & 0.157749 & 3.751998 & 4.339004 \\
\hline 86 & 6 & 0 & -4.302887 & 2.786111 & -0.390964 \\
\hline 87 & 6 & 0 & -5.354095 & 2.374262 & -1.224561 \\
\hline 88 & 6 & 0 & -4.062071 & 4.154686 & -0.218091 \\
\hline 89 & 6 & 0 & -6.171737 & 3.314676 & -1.842215 \\
\hline 90 & 1 & 0 & -5.530127 & 1.304791 & -1.406377 \\
\hline 91 & 6 & 0 & -4.879849 & 5.093170 & -0.845592 \\
\hline 92 & 1 & 0 & -3.231949 & 4.495033 & 0.412982 \\
\hline 93 & 6 & 0 & -5.937150 & 4.676899 & -1.651647 \\
\hline 94 & 1 & 0 & -6.994421 & 2.982270 & -2.485018 \\
\hline 95 & 1 & 0 & -4.687976 & 6.162201 & -0.699915 \\
\hline 96 & 1 & 0 & -6.579014 & 5.417661 & -2.141236 \\
\hline 97 & 1 & 0 & 0.098208 & 2.083824 & -2.305899 \\
\hline
\end{tabular}

Cartesian coordinates (in ̊) and energy of INT-II-Pd-C(5)-E $\mathrm{E}(\mathrm{UM} 06)=-2961.16514043 \mathrm{au}$

\begin{tabular}{|c|c|c|c|c|c|}
\hline \multirow{2}{*}{$\begin{array}{l}\text { Center } \\
\text { number }\end{array}$} & \multirow{2}{*}{$\begin{array}{l}\text { Atomic } \\
\text { number }\end{array}$} & \multirow{2}{*}{$\begin{array}{l}\text { Atomic } \\
\text { type }\end{array}$} & \multicolumn{3}{|c|}{ Coordinates (Angstroms) } \\
\hline & & & $X$ & $\mathrm{Y}$ & $\mathrm{Z}$ \\
\hline 1 & 8 & 0 & 4.745900 & -3.141969 & 0.058898 \\
\hline 2 & 8 & 0 & 0.579038 & 0.994585 & -1.951831 \\
\hline 3 & 1 & 0 & 0.945422 & 1.878341 & -1.775014 \\
\hline 4 & 8 & 0 & -0.122756 & -1.567548 & -1.743893 \\
\hline 5 & 6 & 0 & 5.127365 & -2.012418 & 0.057479 \\
\hline 6 & 6 & 0 & 4.337375 & -0.735175 & -0.316959 \\
\hline 7 & 6 & 0 & 5.403878 & 0.320602 & -0.133677 \\
\hline 8 & 6 & 0 & 5.465318 & 1.695146 & -0.361760 \\
\hline 9 & 1 & 0 & 4.592801 & 2.255718 & -0.720180 \\
\hline 10 & 6 & 0 & 6.654475 & 2.400917 & -0.133719 \\
\hline 11 & 6 & 0 & 7.783209 & 1.700324 & 0.314768 \\
\hline 12 & 1 & 0 & 8.715281 & 2.251941 & 0.489929 \\
\hline 13 & 6 & 0 & 7.755635 & 0.324853 & 0.531476 \\
\hline 14 & 1 & 0 & 8.635665 & -0.232563 & 0.865816 \\
\hline 15 & 6 & 0 & 6.558764 & -0.325362 & 0.293026 \\
\hline 16 & 6 & 0 & 3.877383 & -0.772124 & -1.878138 \\
\hline 17 & 6 & 0 & 2.882386 & 0.322704 & -2.053235 \\
\hline 18 & 1 & 0 & 3.241268 & 1.360679 & -2.048914 \\
\hline 19 & 6 & 0 & 1.567788 & 0.065349 & -2.006705 \\
\hline 20 & 6 & 0 & 1.066331 & -1.329271 & -1.970230 \\
\hline 21 & 6 & 0 & 2.035004 & -2.364321 & -2.276172 \\
\hline 22 & 6 & 0 & 3.364469 & -2.116922 & -2.302802 \\
\hline 23 & 6 & 0 & 3.119717 & -0.621333 & 0.600300 \\
\hline 24 & 6 & 0 & 2.308415 & -1.734424 & 0.863420 \\
\hline 25 & 1 & 0 & 2.613413 & -2.725524 & 0.517153 \\
\hline 26 & 6 & 0 & 1.117266 & -1.599978 & 1.577284 \\
\hline 27 & 1 & 0 & 0.501963 & -2.487583 & 1.770893 \\
\hline 28 & 6 & 0 & 0.711155 & -0.351095 & 2.038648 \\
\hline 29 & 1 & 0 & -0.221308 & -0.246886 & 2.608581 \\
\hline 30 & 6 & 0 & 1.527826 & 0.760381 & 1.818590 \\
\hline 31 & 1 & 0 & 1.238764 & 1.743532 & 2.210847 \\
\hline 32 & 6 & 0 & 2.720202 & 0.621391 & 1.113860 \\
\hline
\end{tabular}




\begin{tabular}{|c|c|c|c|c|c|}
\hline 33 & 1 & 0 & 3.352099 & 1.503793 & 0.969098 \\
\hline 34 & 6 & 0 & 6.723758 & 3.883923 & -0.346372 \\
\hline 35 & 1 & 0 & 5.859901 & 4.257225 & -0.924359 \\
\hline 36 & 1 & 0 & 7.642284 & 4.172999 & -0.888958 \\
\hline 37 & 1 & 0 & 6.737430 & 4.429846 & 0.616840 \\
\hline 38 & 1 & 0 & 1.630193 & -3.357890 & -2.505359 \\
\hline 39 & 6 & 0 & 4.343827 & -3.120859 & -2.809033 \\
\hline 40 & 1 & 0 & 4.028676 & -4.154576 & -2.587147 \\
\hline 41 & 1 & 0 & 4.416763 & -3.018008 & -3.911339 \\
\hline 42 & 1 & 0 & 5.361770 & -2.976260 & -2.409781 \\
\hline 43 & 8 & 0 & 6.389464 & -1.681361 & 0.421397 \\
\hline 44 & 1 & 0 & 4.801500 & -0.549080 & -2.445929 \\
\hline 45 & 46 & 0 & -1.306892 & 0.029571 & -0.554783 \\
\hline 46 & 15 & 0 & -2.863282 & -1.359097 & 0.567730 \\
\hline 47 & 15 & 0 & -2.474009 & 1.835140 & 0.374098 \\
\hline 48 & 6 & 0 & -3.621196 & -0.259485 & 1.855371 \\
\hline 49 & 6 & 0 & -3.944420 & 1.098145 & 1.241559 \\
\hline 50 & 1 & 0 & -4.529295 & -0.734074 & 2.277682 \\
\hline 51 & 1 & 0 & -2.883756 & -0.157220 & 2.676788 \\
\hline 52 & 1 & 0 & -4.353203 & 1.805125 & 1.989615 \\
\hline 53 & 1 & 0 & -4.719039 & 0.989100 & 0.455188 \\
\hline 54 & 6 & 0 & -4.283588 & -1.870707 & -0.457688 \\
\hline 55 & 6 & 0 & -5.239600 & -2.769217 & 0.035557 \\
\hline 56 & 6 & 0 & -4.432473 & -1.335182 & -1.742057 \\
\hline 57 & 6 & 0 & -6.335945 & -3.117733 & -0.747364 \\
\hline 58 & 1 & 0 & -5.122453 & -3.206294 & 1.036715 \\
\hline 59 & 6 & 0 & -5.534269 & -1.684118 & -2.521613 \\
\hline 60 & 1 & 0 & -3.669914 & -0.647004 & -2.134828 \\
\hline 61 & 6 & 0 & -6.484132 & -2.573886 & -2.024357 \\
\hline 62 & 1 & 0 & -7.080476 & -3.822446 & -0.360522 \\
\hline 63 & 1 & 0 & -5.646688 & -1.264364 & -3.527346 \\
\hline 64 & 1 & 0 & -7.347200 & -2.852661 & -2.639282 \\
\hline 65 & 6 & 0 & -2.309275 & -2.861649 & 1.422336 \\
\hline 66 & 6 & 0 & -2.391017 & -3.050733 & 2.806857 \\
\hline 67 & 6 & 0 & -1.703585 & -3.844166 & 0.623490 \\
\hline 68 & 6 & 0 & -1.875884 & -4.212042 & 3.383610 \\
\hline 69 & 1 & 0 & -2.863474 & -2.299887 & 3.451885 \\
\hline 70 & 6 & 0 & -1.201900 & -5.004458 & 1.202989 \\
\hline 71 & 1 & 0 & -1.614321 & -3.684443 & -0.460299 \\
\hline 72 & 6 & 0 & -1.283687 & -5.187922 & 2.584974 \\
\hline 73 & 1 & 0 & -1.944645 & -4.356321 & 4.467603 \\
\hline 74 & 1 & 0 & -0.735766 & -5.770905 & 0.573833 \\
\hline 75 & 1 & 0 & -0.882007 & -6.099184 & 3.041748 \\
\hline 76 & 6 & 0 & -3.190402 & 3.053961 & -0.776897 \\
\hline 77 & 6 & 0 & -3.061382 & 2.843236 & -2.154076 \\
\hline 78 & 6 & 0 & -3.888062 & 4.174805 & -0.306413 \\
\hline 79 & 6 & 0 & -3.631415 & 3.742320 & -3.055151 \\
\hline 80 & 1 & 0 & -2.503865 & 1.968203 & -2.519942 \\
\hline 81 & 6 & 0 & -4.457021 & 5.068211 & -1.208859 \\
\hline 82 & 1 & 0 & -3.981876 & 4.354457 & 0.773286 \\
\hline 83 & 6 & 0 & -4.329313 & 4.851386 & -2.582174 \\
\hline 84 & 1 & 0 & -3.527657 & 3.575837 & -4.133120 \\
\hline 85 & 1 & 0 & -5.003416 & 5.943259 & -0.839620 \\
\hline 86 & 1 & 0 & -4.776936 & 5.558349 & -3.289714 \\
\hline 87 & 6 & 0 & -1.599078 & 2.842456 & 1.619093 \\
\hline 88 & 6 & 0 & -1.802739 & 2.706674 & 2.996560 \\
\hline 89 & 6 & 0 & -0.621015 & 3.737070 & 1.159185 \\
\hline 90 & 6 & 0 & -1.043538 & 3.454547 & 3.897649 \\
\hline 91 & 1 & 0 & -2.564599 & 2.020522 & 3.387047 \\
\hline 92 & 6 & 0 & 0.125835 & 4.490597 & 2.058209 \\
\hline 93 & 1 & 0 & -0.456030 & 3.853595 & 0.077958 \\
\hline 94 & 6 & 0 & -0.081097 & 4.346940 & 3.431914 \\
\hline 95 & 1 & 0 & -1.214480 & 3.343356 & 4.974185 \\
\hline 96 & 1 & 0 & 0.874266 & 5.200084 & 1.687264 \\
\hline 97 & 1 & 0 & 0.508060 & 4.938520 & 4.141211 \\
\hline
\end{tabular}

Cartesian coordinates (in $\AA$ ) and energy of INT-II-Pd-C(5)-F $\mathrm{E}(\mathrm{UM} 06)=-2961.17272627 \mathrm{au}$

Center Atomic Atomic Coordinates (Angstroms)




\begin{tabular}{|c|c|c|c|c|c|}
\hline number & number & type & $\mathrm{X}$ & $\mathrm{Y}$ & $\mathrm{Z}$ \\
\hline 1 & 8 & 0 & 5.138150 & 2.883797 & 0.478557 \\
\hline 2 & 8 & 0 & 0.518158 & 1.680837 & -1.574133 \\
\hline 3 & 1 & 0 & 0.619996 & 2.609314 & -1.298110 \\
\hline 4 & 8 & 0 & 0.502201 & -0.959590 & -1.914029 \\
\hline 5 & 6 & 0 & 4.441894 & 1.942406 & 0.686554 \\
\hline 6 & 6 & 0 & 4.527202 & 0.537930 & 0.048225 \\
\hline 7 & 6 & 0 & 3.391206 & -0.162424 & 0.734881 \\
\hline 8 & 6 & 0 & 2.856171 & -1.441995 & 0.628145 \\
\hline 9 & 1 & 0 & 3.366532 & -2.215188 & 0.039611 \\
\hline 10 & 6 & 0 & 1.641954 & -1.756340 & 1.258270 \\
\hline 11 & 6 & 0 & 0.997870 & -0.764916 & 2.010500 \\
\hline 12 & 1 & 0 & 0.054430 & -1.012984 & 2.514257 \\
\hline 13 & 6 & 0 & 1.538439 & 0.516052 & 2.166503 \\
\hline 14 & 1 & 0 & 1.047461 & 1.279092 & 2.779099 \\
\hline 15 & 6 & 0 & 2.726489 & 0.780870 & 1.513494 \\
\hline 16 & 6 & 0 & 4.157584 & 0.784619 & -1.510120 \\
\hline 17 & 6 & 0 & 2.923534 & 1.622341 & -1.557479 \\
\hline 18 & 1 & 0 & 3.018853 & 2.693466 & -1.329795 \\
\hline 19 & 6 & 0 & 1.715389 & 1.058161 & -1.695200 \\
\hline 20 & 6 & 0 & 1.589462 & -0.384816 & -2.001618 \\
\hline 21 & 6 & 0 & 2.802560 & -1.051110 & -2.441231 \\
\hline 22 & 6 & 0 & 4.031216 & -0.522350 & -2.236894 \\
\hline 23 & 6 & 0 & 5.921901 & -0.035527 & 0.222734 \\
\hline 24 & 6 & 0 & 7.025126 & 0.623939 & -0.336732 \\
\hline 25 & 1 & 0 & 6.891091 & 1.577623 & -0.859552 \\
\hline 26 & 6 & 0 & 8.304928 & 0.091905 & -0.217585 \\
\hline 27 & 1 & 0 & 9.154363 & 0.622065 & -0.662414 \\
\hline 28 & 6 & 0 & 8.508300 & -1.104977 & 0.468815 \\
\hline 29 & 1 & 0 & 9.515896 & -1.525928 & 0.557471 \\
\hline 30 & 6 & 0 & 7.423958 & -1.750547 & 1.055792 \\
\hline 31 & 1 & 0 & 7.573808 & -2.679364 & 1.617504 \\
\hline 32 & 6 & 0 & 6.140868 & -1.216258 & 0.939737 \\
\hline 33 & 1 & 0 & 5.303197 & -1.729718 & 1.423668 \\
\hline 34 & 6 & 0 & 1.036017 & -3.115948 & 1.081857 \\
\hline 35 & 1 & 0 & 0.504784 & -3.191049 & 0.109600 \\
\hline 36 & 1 & 0 & 0.301703 & -3.345375 & 1.875127 \\
\hline 37 & 1 & 0 & 1.807041 & -3.908051 & 1.089454 \\
\hline 38 & 1 & 0 & 2.672801 & -2.039066 & -2.900853 \\
\hline 39 & 6 & 0 & 5.266507 & -1.241491 & -2.659145 \\
\hline 40 & 1 & 0 & 5.824259 & -1.640718 & -1.788321 \\
\hline 41 & 1 & 0 & 5.025979 & -2.085435 & -3.328305 \\
\hline 42 & 1 & 0 & 5.965996 & -0.561126 & -3.180469 \\
\hline 43 & 8 & 0 & 3.354417 & 2.002061 & 1.510282 \\
\hline 44 & 1 & 0 & 5.011883 & 1.359079 & -1.913958 \\
\hline 45 & 46 & 0 & -1.177086 & 0.063127 & -0.588298 \\
\hline 46 & 15 & 0 & -2.804387 & -1.555211 & 0.044830 \\
\hline 47 & 15 & 0 & -2.762757 & 1.667683 & 0.030924 \\
\hline 48 & 6 & 0 & -4.297476 & -0.579397 & 0.588624 \\
\hline 49 & 6 & 0 & -4.374678 & 0.771812 & -0.106262 \\
\hline 50 & 1 & 0 & -4.212503 & -0.453577 & 1.684991 \\
\hline 51 & 1 & 0 & -5.213957 & -1.173271 & 0.413375 \\
\hline 52 & 1 & 0 & -5.210453 & 1.390332 & 0.275435 \\
\hline 53 & 1 & 0 & -4.545309 & 0.648954 & -1.195684 \\
\hline 54 & 6 & 0 & -3.359228 & -2.559319 & -1.372504 \\
\hline 55 & 6 & 0 & -4.586241 & -3.238619 & -1.366840 \\
\hline 56 & 6 & 0 & -2.510499 & -2.674717 & -2.480346 \\
\hline 57 & 6 & 0 & -4.962466 & -4.011706 & -2.460737 \\
\hline 58 & 1 & 0 & -5.251327 & -3.183602 & -0.494699 \\
\hline 59 & 6 & 0 & -2.890730 & -3.454448 & -3.572814 \\
\hline 60 & 1 & 0 & -1.541738 & -2.156074 & -2.485440 \\
\hline 61 & 6 & 0 & -4.115323 & -4.117695 & -3.565162 \\
\hline 62 & 1 & 0 & -5.922490 & -4.539741 & -2.450989 \\
\hline 63 & 1 & 0 & -2.223252 & -3.541605 & -4.437196 \\
\hline 64 & 1 & 0 & -4.414293 & -4.726815 & -4.425614 \\
\hline 65 & 6 & 0 & -2.555328 & -2.724359 & 1.423017 \\
\hline 66 & 6 & 0 & -2.355894 & -2.209613 & 2.713578 \\
\hline 67 & 6 & 0 & -2.469820 & -4.106220 & 1.216572 \\
\hline 68 & 6 & 0 & -2.100888 & -3.065115 & 3.781308 \\
\hline 69 & 1 & 0 & -2.394470 & -1.124064 & 2.890730 \\
\hline 70 & 6 & 0 & -2.210398 & -4.959169 & 2.288497 \\
\hline
\end{tabular}




$\begin{array}{rrrrrr}71 & 1 & 0 & -2.609619 & -4.524508 & 0.211993 \\ 72 & 6 & 0 & -2.028100 & -4.443061 & 3.569928 \\ 73 & 1 & 0 & -1.956541 & -2.654111 & 4.786854 \\ 74 & 1 & 0 & -2.153634 & -6.040205 & 2.118738 \\ 75 & 1 & 0 & -1.828003 & -5.116820 & 4.410394 \\ 76 & 6 & 0 & -2.639935 & 2.281959 & 1.740893 \\ 77 & 6 & 0 & -1.396174 & 2.795105 & 2.136006 \\ 78 & 6 & 0 & -3.698346 & 2.275224 & 2.656320 \\ 79 & 6 & 0 & -1.215126 & 3.302130 & 3.418747 \\ 80 & 1 & 0 & -0.560779 & 2.798236 & 1.419908 \\ 81 & 6 & 0 & -3.511155 & 2.770665 & 3.947160 \\ 82 & 1 & 0 & -4.685793 & 1.891782 & 2.370384 \\ 83 & 6 & 0 & -2.273641 & 3.284610 & 4.328590 \\ 84 & 1 & 0 & -0.241934 & 3.711752 & 3.713439 \\ 85 & 1 & 0 & -4.344667 & 2.761297 & 4.658292 \\ 86 & 1 & 0 & -2.132100 & 3.677101 & 5.341604 \\ 87 & 6 & 0 & -2.948318 & 3.150291 & -1.011403 \\ 88 & 6 & 0 & -2.653012 & 3.029841 & -2.376965 \\ 89 & 6 & 0 & -3.383889 & 4.379522 & -0.500490 \\ 90 & 6 & 0 & -2.810514 & 4.123125 & -3.224850 \\ 91 & 1 & 0 & -2.288985 & 2.070484 & -2.773110 \\ 92 & 6 & 0 & -3.531226 & 5.471919 & -1.352504 \\ 93 & 1 & 0 & -3.609719 & 4.487133 & 0.568249 \\ 94 & 6 & 0 & -3.248013 & 5.344402 & -2.712030 \\ 95 & 1 & 0 & -2.583712 & 4.023690 & -4.292208 \\ 96 & 1 & 0 & -3.872612 & 6.432407 & -0.950607 \\ 97 & 1 & 0 & -3.365380 & 6.206677 & -3.377864 \\ -------------------------------------------------------\end{array}$

Cartesian coordinates (in $\AA$ ) and energy of INT-I-C(6)-A $\mathrm{E}(\mathrm{UM} 06)=-1149.02063812 \mathrm{au}$

\begin{tabular}{|c|c|c|c|c|c|}
\hline \multirow{2}{*}{$\begin{array}{l}\text { Center } \\
\text { number }\end{array}$} & \multirow{2}{*}{$\begin{array}{l}\text { Atomic } \\
\text { number }\end{array}$} & \multirow{2}{*}{$\begin{array}{l}\text { Atomic } \\
\text { type }\end{array}$} & \multicolumn{3}{|c|}{ Coordinates (Angstroms) } \\
\hline & & & $\mathrm{X}$ & $\mathrm{Y}$ & Z \\
\hline 1 & 8 & 0 & 2.170442 & 2.286919 & 0.445392 \\
\hline 2 & 1 & 0 & 2.084238 & 2.027272 & 1.390729 \\
\hline 3 & 8 & 0 & 1.289725 & 0.865998 & 2.675137 \\
\hline 4 & 6 & 0 & 4.062639 & -1.433178 & 0.400517 \\
\hline 5 & 1 & 0 & 5.019656 & -1.315056 & 0.921669 \\
\hline 6 & 6 & 0 & 2.893755 & -1.001401 & 1.013545 \\
\hline 7 & 1 & 0 & 2.934721 & -0.551828 & 2.009309 \\
\hline 8 & 6 & 0 & 4.024453 & -2.020208 & -0.865518 \\
\hline 9 & 1 & 0 & 4.949902 & -2.357728 & -1.346035 \\
\hline 10 & 6 & 0 & 2.800110 & -2.165339 & -1.518452 \\
\hline 11 & 1 & 0 & 2.760530 & -2.601463 & -2.522838 \\
\hline 12 & 6 & 0 & 1.628967 & -1.733531 & -0.912796 \\
\hline 13 & 1 & 0 & 0.689618 & -1.799503 & -1.468944 \\
\hline 14 & 6 & 0 & 1.642710 & -1.147567 & 0.371205 \\
\hline 15 & 6 & 0 & -0.239321 & 2.624588 & 0.374330 \\
\hline 16 & 6 & 0 & 0.961187 & 2.180602 & -0.168580 \\
\hline 17 & 6 & 0 & 0.965803 & 1.565770 & -1.436506 \\
\hline 18 & 6 & 0 & -1.449262 & 1.795032 & -1.562043 \\
\hline 19 & 6 & 0 & -1.437068 & 2.424470 & -0.313138 \\
\hline 20 & 1 & 0 & -2.381414 & 2.759519 & 0.134350 \\
\hline 21 & 6 & 0 & -0.231467 & 1.383889 & -2.119789 \\
\hline 22 & 1 & 0 & -0.197206 & 0.886251 & -3.097761 \\
\hline 23 & 6 & 0 & -1.699043 & -0.252367 & 1.656807 \\
\hline 24 & 6 & 0 & -0.940873 & -0.989026 & 0.720347 \\
\hline 25 & 6 & 0 & -1.635272 & -1.802963 & -0.198612 \\
\hline 26 & 1 & 0 & -1.095597 & -2.430721 & -0.915346 \\
\hline 27 & 6 & 0 & -3.026518 & -1.836279 & -0.191405 \\
\hline 28 & 6 & 0 & -3.732023 & -1.062951 & 0.754494 \\
\hline 29 & 1 & 0 & -4.829156 & -1.100927 & 0.753119 \\
\hline 30 & 6 & 0 & -3.079374 & -0.269578 & 1.697337 \\
\hline 31 & 1 & 0 & -3.622844 & 0.319030 & 2.442828 \\
\hline 32 & 6 & 0 & -2.733353 & 1.535565 & -2.291106 \\
\hline 33 & 1 & 0 & -2.725498 & 1.976427 & -3.306509 \\
\hline 34 & 1 & 0 & -3.600218 & 1.953075 & -1.748221 \\
\hline
\end{tabular}




$\begin{array}{lllrrr}35 & 1 & 0 & -2.909403 & 0.448041 & -2.414234 \\ 36 & 6 & 0 & 0.425601 & -0.694787 & 1.002977 \\ 37 & 6 & 0 & 0.399801 & 0.257650 & 2.122499 \\ 38 & 8 & 0 & 2.126750 & 1.133446 & -1.957905 \\ 39 & 1 & 0 & 2.818032 & 1.271433 & -1.279313 \\ 40 & 6 & 0 & -3.786186 & -2.671549 & -1.179329 \\ 41 & 1 & 0 & -4.563439 & -3.282426 & -0.683164 \\ 42 & 1 & 0 & -4.304887 & -2.039991 & -1.927302 \\ 43 & 1 & 0 & -3.119881 & -3.357226 & -1.731700 \\ 44 & 8 & 0 & -0.908343 & 0.459773 & 2.498454 \\ 45 & 1 & 0 & -0.227335 & 3.105686 & 1.360034\end{array}$

Cartesian coordinates (in $\AA$ ) and energy of INT-I-C(6)-B $\mathrm{E}(\mathrm{UM} 06)=-1149.01538743$

\begin{tabular}{|c|c|c|c|c|c|}
\hline \multirow{2}{*}{$\begin{array}{l}\text { Center } \\
\text { number }\end{array}$} & \multirow{2}{*}{$\begin{array}{l}\text { Atomic } \\
\text { number }\end{array}$} & \multirow{2}{*}{$\begin{array}{l}\text { Atomic } \\
\text { type }\end{array}$} & \multicolumn{3}{|c|}{ Coordinates (Angstroms) } \\
\hline & & & $\mathrm{X}$ & $\mathrm{Y}$ & Z \\
\hline 1 & 8 & 0 & 1.574111 & 0.928017 & 2.146611 \\
\hline 2 & 1 & 0 & 2.312805 & 0.405459 & 1.783842 \\
\hline 3 & 8 & 0 & 1.135847 & -3.055389 & -1.112137 \\
\hline 4 & 6 & 0 & 4.319829 & -0.440690 & 0.159397 \\
\hline 5 & 1 & 0 & 5.050868 & -1.087089 & 0.659001 \\
\hline 6 & 6 & 0 & 3.075044 & -0.955912 & -0.187416 \\
\hline 7 & 1 & 0 & 2.829502 & -2.001394 & 0.024189 \\
\hline 8 & 6 & 0 & 4.645944 & 0.886237 & -0.132455 \\
\hline 9 & 1 & 0 & 5.628532 & 1.286510 & 0.141671 \\
\hline 10 & 6 & 0 & 3.717076 & 1.691630 & -0.792912 \\
\hline 11 & 1 & 0 & 3.972388 & 2.725877 & -1.050367 \\
\hline 12 & 6 & 0 & 2.467962 & 1.187242 & -1.132845 \\
\hline 13 & 1 & 0 & 1.762776 & 1.821315 & -1.679889 \\
\hline 14 & 6 & 0 & 2.109969 & -0.141560 & -0.823017 \\
\hline 15 & 6 & 0 & 0.309518 & -1.156900 & 2.023136 \\
\hline 16 & 6 & 0 & 0.404222 & 0.229825 & 2.049777 \\
\hline 17 & 6 & 0 & -0.758078 & 1.007745 & 1.952482 \\
\hline 18 & 6 & 0 & -2.100691 & -1.008259 & 1.765572 \\
\hline 19 & 6 & 0 & -0.933185 & -1.771557 & 1.868017 \\
\hline 20 & 1 & 0 & -0.991452 & -2.866152 & 1.814459 \\
\hline 21 & 6 & 0 & -1.995284 & 0.386234 & 1.824222 \\
\hline 22 & 1 & 0 & -2.884315 & 1.023085 & 1.727871 \\
\hline 23 & 6 & 0 & -1.404621 & -0.922638 & -1.607439 \\
\hline 24 & 6 & 0 & -0.426897 & 0.061706 & -1.326716 \\
\hline 25 & 6 & 0 & -0.836645 & 1.401695 & -1.207499 \\
\hline 26 & 1 & 0 & -0.131642 & 2.178601 & -0.888956 \\
\hline 27 & 6 & 0 & -2.172779 & 1.743441 & -1.404201 \\
\hline 28 & 6 & 0 & -3.094497 & 0.735189 & -1.743403 \\
\hline 29 & 1 & 0 & -4.143350 & 1.013949 & -1.907343 \\
\hline 30 & 6 & 0 & -2.727200 & -0.611526 & -1.842637 \\
\hline 31 & 1 & 0 & -3.455151 & -1.396689 & -2.069642 \\
\hline 32 & 6 & 0 & -3.435192 & -1.673485 & 1.604268 \\
\hline 33 & 1 & 0 & -3.835856 & -2.028864 & 2.574376 \\
\hline 34 & 1 & 0 & -3.363316 & -2.556458 & 0.941137 \\
\hline 35 & 1 & 0 & -4.184168 & -0.983809 & 1.173936 \\
\hline 36 & 6 & 0 & 0.788099 & -0.642430 & -1.117116 \\
\hline 37 & 6 & 0 & 0.458772 & -2.074299 & -1.246704 \\
\hline 38 & 8 & 0 & -0.664096 & 2.357606 & 1.932133 \\
\hline 39 & 1 & 0 & 0.282065 & 2.583467 & 2.012271 \\
\hline 40 & 6 & 0 & -2.612361 & 3.161561 & -1.189977 \\
\hline 41 & 1 & 0 & -3.699615 & 3.282733 & -1.340347 \\
\hline 42 & 1 & 0 & -2.369815 & 3.486311 & -0.159011 \\
\hline 43 & 1 & 0 & -2.099423 & 3.859618 & -1.878774 \\
\hline 44 & 8 & 0 & -0.883245 & -2.173595 & -1.583629 \\
\hline 45 & 1 & 0 & 1.224185 & -1.759076 & 2.102568 \\
\hline
\end{tabular}

Cartesian coordinates (in Å) and energy of INT-I-C(6)-C $\mathrm{E}(\mathrm{UM} 06)=-1149.01014219 \mathrm{au}$

Center Atomic Atomic Coordinates (Angstroms)




\begin{tabular}{|c|c|c|c|c|c|}
\hline number & number & type & $\mathrm{X}$ & $\mathrm{Y}$ & $\mathrm{Z}$ \\
\hline 1 & 8 & 0 & -2.474808 & -0.773291 & -1.981647 \\
\hline 2 & 1 & 0 & -1.883039 & -1.532630 & -1.845346 \\
\hline 3 & 8 & 0 & 1.586463 & 1.885930 & 1.968935 \\
\hline 4 & 6 & 0 & 3.797352 & -1.594275 & -1.489301 \\
\hline 5 & 1 & 0 & 3.852497 & -2.271786 & -2.349169 \\
\hline 6 & 6 & 0 & 2.577439 & -1.375327 & -0.863655 \\
\hline 7 & 1 & 0 & 1.680119 & -1.861400 & -1.259721 \\
\hline 8 & 6 & 0 & 4.946816 & -0.946673 & -1.033415 \\
\hline 9 & 1 & 0 & 5.910384 & -1.122187 & -1.525312 \\
\hline 10 & 6 & 0 & 4.857550 & -0.067222 & 0.046337 \\
\hline 11 & 1 & 0 & 5.754146 & 0.449624 & 0.407291 \\
\hline 12 & 6 & 0 & 3.639141 & 0.162464 & 0.672162 \\
\hline 13 & 1 & 0 & 3.575699 & 0.858302 & 1.513057 \\
\hline 14 & 6 & 0 & 2.468959 & -0.498954 & 0.237998 \\
\hline 15 & 6 & 0 & -0.500095 & 0.657211 & -1.915961 \\
\hline 16 & 6 & 0 & -1.824296 & 0.378218 & -1.612100 \\
\hline 17 & 6 & 0 & -2.582968 & 1.300757 & -0.874486 \\
\hline 18 & 6 & 0 & -0.651911 & 2.767181 & -0.720809 \\
\hline 19 & 6 & 0 & 0.089907 & 1.837440 & -1.460390 \\
\hline 20 & 1 & 0 & 1.146295 & 2.038588 & -1.682358 \\
\hline 21 & 6 & 0 & -1.995958 & 2.488103 & -0.448283 \\
\hline 22 & 1 & 0 & -2.607736 & 3.184902 & 0.137667 \\
\hline 23 & 6 & 0 & -0.946412 & -0.275064 & 1.606896 \\
\hline 24 & 6 & 0 & 0.000123 & -1.029249 & 0.875109 \\
\hline 25 & 6 & 0 & -0.406480 & -2.270962 & 0.338932 \\
\hline 26 & 1 & 0 & 0.309300 & -2.919023 & -0.180512 \\
\hline 27 & 6 & 0 & -1.718357 & -2.705288 & 0.505724 \\
\hline 28 & 6 & 0 & -2.629716 & -1.897929 & 1.222871 \\
\hline 29 & 1 & 0 & -3.662811 & -2.250131 & 1.342930 \\
\hline 30 & 6 & 0 & -2.255933 & -0.680812 & 1.787885 \\
\hline 31 & 1 & 0 & -2.961897 & -0.052054 & 2.338849 \\
\hline 32 & 6 & 0 & -0.005200 & 4.002880 & -0.173343 \\
\hline 33 & 1 & 0 & -0.735478 & 4.821139 & -0.039032 \\
\hline 34 & 1 & 0 & 0.802785 & 4.365601 & -0.834448 \\
\hline 35 & 1 & 0 & 0.452659 & 3.796992 & 0.815827 \\
\hline 36 & 6 & 0 & 1.200632 & -0.268733 & 0.888412 \\
\hline 37 & 6 & 0 & 0.905718 & 0.940445 & 1.679330 \\
\hline 38 & 8 & 0 & -3.862840 & 1.015835 & -0.560983 \\
\hline 39 & 1 & 0 & -4.052451 & 0.127145 & -0.917989 \\
\hline 40 & 6 & 0 & -2.189272 & -4.004370 & -0.079586 \\
\hline 41 & 1 & 0 & -2.932826 & -3.836993 & -0.885028 \\
\hline 42 & 1 & 0 & -1.355334 & -4.587952 & -0.508355 \\
\hline 43 & 1 & 0 & -2.686115 & -4.636797 & 0.679878 \\
\hline 44 & 8 & 0 & -0.417123 & 0.868999 & 2.092676 \\
\hline 45 & 1 & 0 & 0.078027 & -0.070178 & -2.502062 \\
\hline
\end{tabular}

Cartesian coordinates (in $\AA$ ) and energy of INT-I-C(6)-D

$\mathrm{E}(\mathrm{UM} 06)=-1149.01441607 \mathrm{au}$

\begin{tabular}{cccccc}
$\begin{array}{c}\text { Center } \\
\text { number }\end{array}$ & $\begin{array}{c}\text { Atomic } \\
\text { number }\end{array}$ & $\begin{array}{c}\text { Atomic } \\
\text { type }\end{array}$ & \multicolumn{3}{c}{ Coordinates (Angstroms) } \\
\hline 1 & 8 & 0 & Y & $\mathrm{Z}$ \\
2 & 1 & 0 & 2.715285 & -1.142994 & 1.089369 \\
3 & 8 & 0 & 1.506602 & -1.366500 & -2.362111 \\
4 & 6 & 0 & 4.329851 & 1.038780 & -0.318250 \\
5 & 1 & 0 & 5.297423 & 0.732522 & -0.732339 \\
6 & 6 & 0 & 3.158941 & 0.604754 & -0.929271 \\
7 & 1 & 0 & 3.207518 & -0.046318 & -1.805851 \\
8 & 6 & 0 & 4.281364 & 1.858456 & 0.810538 \\
9 & 1 & 0 & 5.207104 & 2.196555 & 1.289941 \\
10 & 6 & 0 & 3.040918 & 2.242549 & 1.324136 \\
11 & 1 & 0 & 2.988205 & 2.878737 & 2.215244 \\
12 & 6 & 0 & 1.867189 & 1.814906 & 0.716643 \\
13 & 1 & 0 & 0.908186 & 2.089931 & 1.163847 \\
14 & 6 & 0 & 1.893486 & 0.984416 & -0.425074 \\
15 & 6 & 0 & 0.458746 & -0.779164 & 2.192553 \\
16 & 6 & 0 & 0.863748 & -1.673147 & 1.208068
\end{tabular}




$\begin{array}{lllrrr}17 & 6 & 0 & -0.101625 & -2.405444 & 0.491027 \\ 18 & 6 & 0 & -1.871011 & -1.280231 & 1.729065 \\ 19 & 6 & 0 & -0.897734 & -0.575153 & 2.445307 \\ 20 & 1 & 0 & -1.204887 & 0.142693 & 3.216636 \\ 21 & 6 & 0 & -1.451786 & -2.201873 & 0.762976 \\ 22 & 1 & 0 & -2.179572 & -2.771862 & 0.170919 \\ 23 & 6 & 0 & -1.443933 & 0.078817 & -1.716134 \\ 24 & 6 & 0 & -0.676799 & 0.936963 & -0.893452 \\ 25 & 6 & 0 & -1.354096 & 1.949047 & -0.181543 \\ 26 & 1 & 0 & -0.807271 & 2.663310 & 0.443035 \\ 27 & 6 & 0 & -2.737613 & 2.067698 & -0.276043 \\ 28 & 6 & 0 & -3.451040 & 1.182017 & -1.109861 \\ 29 & 1 & 0 & -4.540974 & 1.292040 & -1.185356 \\ 30 & 6 & 0 & -2.815014 & 0.180183 & -1.844030 \\ 31 & 1 & 0 & -3.364139 & -0.503786 & -2.498342 \\ 32 & 6 & 0 & -3.332265 & -1.031217 & 1.950723 \\ 33 & 1 & 0 & -3.772685 & -0.470942 & 1.099709 \\ 34 & 1 & 0 & -3.510107 & -0.438259 & 2.866372 \\ 35 & 1 & 0 & -3.900938 & -1.975812 & 2.041624 \\ 36 & 6 & 0 & 0.673514 & 0.506078 & -1.035034 \\ 37 & 6 & 0 & 0.634916 & -0.639614 & -1.963725 \\ 38 & 8 & 0 & 0.266892 & -3.281356 & -0.459355 \\ 39 & 1 & 0 & 1.195961 & -3.111686 & -0.709929 \\ 40 & 6 & 0 & -3.480867 & 3.098067 & 0.521301 \\ 41 & 1 & 0 & -3.989706 & 2.638592 & 1.392546 \\ 42 & 1 & 0 & -2.804970 & 3.879511 & 0.911378 \\ 43 & 1 & 0 & -4.263289 & 3.595893 & -0.080984 \\ 44 & 8 & 0 & -0.676394 & -0.833557 & -2.353985 \\ 45 & 1 & 0 & 1.224884 & -0.234607 & 2.760356 \\ ----------------------------------------------------------------------------\end{array}$

Cartesian coordinates (in Å) and energy of INT-I-C(6)-E $\mathrm{E}(\mathrm{UM} 06)=-1149.01332535 \mathrm{au}$

\begin{tabular}{|c|c|c|c|c|c|}
\hline \multirow{2}{*}{$\begin{array}{l}\text { Center } \\
\text { number }\end{array}$} & \multirow{2}{*}{$\begin{array}{l}\text { Atomic } \\
\text { number }\end{array}$} & \multirow{2}{*}{$\begin{array}{l}\text { Atomic } \\
\text { type }\end{array}$} & \multicolumn{3}{|c|}{ Coordinates (Angstroms) } \\
\hline & & & $\mathrm{X}$ & $\mathrm{Y}$ & $\mathrm{Z}$ \\
\hline 1 & 8 & 0 & 1.454011 & -2.360375 & 1.253221 \\
\hline 2 & 1 & 0 & 2.276590 & -1.864223 & 1.101601 \\
\hline 3 & 8 & 0 & -1.018760 & 3.168284 & -0.019556 \\
\hline 4 & 6 & 0 & -2.350413 & -1.657621 & -2.010294 \\
\hline 5 & 1 & 0 & -2.316115 & -2.745506 & -2.135771 \\
\hline 6 & 6 & 0 & -1.209523 & -0.982325 & -1.602881 \\
\hline 7 & 1 & 0 & -0.310328 & -1.562469 & -1.378990 \\
\hline 8 & 6 & 0 & -3.540910 & -0.960182 & -2.220124 \\
\hline 9 & 1 & 0 & -4.443646 & -1.493757 & -2.539333 \\
\hline 10 & 6 & 0 & -3.576942 & 0.420581 & -2.011659 \\
\hline 11 & 1 & 0 & -4.508306 & 0.975052 & -2.174513 \\
\hline 12 & 6 & 0 & -2.441104 & 1.101856 & -1.597165 \\
\hline 13 & 1 & 0 & -2.479575 & 2.181143 & -1.427561 \\
\hline 14 & 6 & 0 & -1.223526 & 0.413464 & -1.384248 \\
\hline 15 & 6 & 0 & 0.666613 & -0.234571 & 2.158033 \\
\hline 16 & 6 & 0 & 0.455065 & -1.494386 & 1.612520 \\
\hline 17 & 6 & 0 & -0.850902 & -1.940494 & 1.371013 \\
\hline 18 & 6 & 0 & -1.725056 & 0.194809 & 2.131586 \\
\hline 19 & 6 & 0 & -0.414960 & 0.610801 & 2.403131 \\
\hline 20 & 1 & 0 & -0.238089 & 1.619116 & 2.799942 \\
\hline 21 & 6 & 0 & -1.925898 & -1.094801 & 1.631755 \\
\hline 22 & 1 & 0 & -2.933599 & -1.457322 & 1.391689 \\
\hline 23 & 6 & 0 & 1.998882 & 1.717961 & -0.157115 \\
\hline 24 & 6 & 0 & 1.310884 & 0.689492 & -0.843075 \\
\hline 25 & 6 & 0 & 2.045617 & -0.434707 & -1.258009 \\
\hline 26 & 1 & 0 & 1.569747 & -1.242403 & -1.825123 \\
\hline 27 & 6 & 0 & 3.405645 & -0.540203 & -0.955005 \\
\hline 28 & 6 & 0 & 4.035386 & 0.502225 & -0.250514 \\
\hline 29 & 1 & 0 & 5.103961 & 0.413618 & -0.016563 \\
\hline 30 & 6 & 0 & 3.343356 & 1.651747 & 0.145779 \\
\hline 31 & 1 & 0 & 3.832742 & 2.467189 & 0.687180 \\
\hline 32 & 6 & 0 & -2.872553 & 1.137311 & 2.337672 \\
\hline 33 & 1 & 0 & -3.831792 & 0.684894 & 2.028690 \\
\hline 34 & 1 & 0 & -2.725748 & 2.062447 & 1.746367 \\
\hline
\end{tabular}




$\begin{array}{rrrrrr}35 & 1 & 0 & -2.967906 & 1.442067 & 3.397767 \\ 36 & 6 & 0 & -0.054533 & 1.102329 & -0.903734 \\ 37 & 6 & 0 & -0.107283 & 2.418969 & -0.237258 \\ 38 & 8 & 0 & -1.056348 & -3.163627 & 0.832128 \\ 39 & 1 & 0 & -0.185318 & -3.587224 & 0.721469 \\ 40 & 6 & 0 & 4.176931 & -1.751255 & -1.396049 \\ 41 & 1 & 0 & 5.156524 & -1.819903 & -0.890504 \\ 42 & 1 & 0 & 3.618898 & -2.683773 & -1.185086 \\ 43 & 1 & 0 & 4.371522 & -1.735426 & -2.485995 \\ 44 & 8 & 0 & 1.176857 & 2.733650 & 0.189557 \\ 45 & 1 & 0 & 1.695696 & 0.097015 & 2.355778\end{array}$

Cartesian coordinates (in $\AA$ ) and energy of INT-I-C(6)-F $\mathrm{E}(\mathrm{UM} 06)=-1149.01721829 \mathrm{au}$

\begin{tabular}{|c|c|c|c|c|c|}
\hline \multirow{2}{*}{$\begin{array}{l}\text { Center } \\
\text { number }\end{array}$} & \multirow{2}{*}{$\begin{array}{l}\text { Atomic } \\
\text { number }\end{array}$} & \multirow{2}{*}{$\begin{array}{l}\text { Atomic } \\
\text { type }\end{array}$} & \multicolumn{3}{|c|}{ Coordinates (Angstroms) } \\
\hline & & & $\mathrm{X}$ & $\mathrm{Y}$ & $\mathrm{Z}$ \\
\hline 1 & 8 & 0 & -0.983466 & -3.465116 & -0.216345 \\
\hline 2 & 1 & 0 & -0.210638 & -3.389933 & -0.819384 \\
\hline 3 & 8 & 0 & 1.016464 & -2.211760 & -1.751968 \\
\hline 4 & 6 & 0 & 4.160170 & 1.736823 & 0.246836 \\
\hline 5 & 1 & 0 & 4.548803 & 2.749836 & 0.401816 \\
\hline 6 & 6 & 0 & 2.871596 & 1.562178 & -0.239289 \\
\hline 7 & 1 & 0 & 2.273326 & 2.441926 & -0.496133 \\
\hline 8 & 6 & 0 & 4.962582 & 0.628889 & 0.523801 \\
\hline 9 & 1 & 0 & 5.978651 & 0.766767 & 0.910886 \\
\hline 10 & 6 & 0 & 4.467226 & -0.655114 & 0.290340 \\
\hline 11 & 1 & 0 & 5.095128 & -1.529910 & 0.494598 \\
\hline 12 & 6 & 0 & 3.182065 & -0.837165 & -0.202614 \\
\hline 13 & 1 & 0 & 2.803791 & -1.845106 & -0.393838 \\
\hline 14 & 6 & 0 & 2.345762 & 0.271416 & -0.462891 \\
\hline 15 & 6 & 0 & 0.073081 & -2.033038 & 1.448220 \\
\hline 16 & 6 & 0 & -0.997978 & -2.406640 & 0.647025 \\
\hline 17 & 6 & 0 & -2.186003 & -1.653530 & 0.680942 \\
\hline 18 & 6 & 0 & -1.196895 & -0.156055 & 2.318532 \\
\hline 19 & 6 & 0 & -0.021320 & -0.909145 & 2.273938 \\
\hline 20 & 1 & 0 & 0.839327 & -0.613926 & 2.887654 \\
\hline 21 & 6 & 0 & -2.278884 & -0.547167 & 1.517625 \\
\hline 22 & 1 & 0 & -3.211555 & 0.032020 & 1.508120 \\
\hline 23 & 6 & 0 & -1.154612 & 0.337793 & -1.617592 \\
\hline 24 & 6 & 0 & -0.105224 & 0.996519 & -0.940557 \\
\hline 25 & 6 & 0 & -0.371396 & 2.254709 & -0.361756 \\
\hline 26 & 1 & 0 & 0.389455 & 2.769846 & 0.235600 \\
\hline 27 & 6 & 0 & -1.628169 & 2.833415 & -0.505427 \\
\hline 28 & 6 & 0 & -2.627707 & 2.144775 & -1.226407 \\
\hline 29 & 1 & 0 & -3.616592 & 2.610450 & -1.328667 \\
\hline 30 & 6 & 0 & -2.410342 & 0.884862 & -1.784038 \\
\hline 31 & 1 & 0 & -3.198456 & 0.328927 & -2.299856 \\
\hline 32 & 6 & 0 & -1.317759 & 1.054844 & 3.195553 \\
\hline 33 & 1 & 0 & -1.692877 & 1.928230 & 2.626484 \\
\hline 34 & 1 & 0 & -0.345604 & 1.332640 & 3.641177 \\
\hline 35 & 1 & 0 & -2.028298 & 0.886790 & 4.028490 \\
\hline 36 & 6 & 0 & 0.999251 & 0.098765 & -0.953087 \\
\hline 37 & 6 & 0 & 0.505264 & -1.126589 & -1.595046 \\
\hline 38 & 8 & 0 & -3.202005 & -1.992014 & -0.134719 \\
\hline 39 & 1 & 0 & -2.884283 & -2.751111 & -0.665167 \\
\hline 40 & 6 & 0 & -1.941656 & 4.168190 & 0.103256 \\
\hline 41 & 1 & 0 & -2.131349 & 4.934561 & -0.673090 \\
\hline 42 & 1 & 0 & -2.849476 & 4.122023 & 0.734480 \\
\hline 43 & 1 & 0 & -1.111859 & 4.533035 & 0.734172 \\
\hline 44 & 8 & 0 & -0.782973 & -0.902506 & -2.031761 \\
\hline 45 & 1 & 0 & 0.994038 & -2.628307 & 1.406800 \\
\hline
\end{tabular}

Cartesian coordinates (in $\AA$ ) and energy of TS-C(6)-A $\mathrm{E}(\mathrm{UM} 06)=-1148.97801311 \mathrm{au}$

Center Atomic Atomic Coordinates (Angstroms)




\begin{tabular}{|c|c|c|c|c|c|}
\hline number & number & type & $\mathrm{X}$ & $\mathrm{Y}$ & $\mathrm{Z}$ \\
\hline 1 & 8 & 0 & -2.388398 & -1.544141 & -1.661721 \\
\hline 2 & 1 & 0 & -1.718787 & -2.249026 & -1.474113 \\
\hline 3 & 8 & 0 & -0.314978 & -3.034817 & -0.830237 \\
\hline 4 & 6 & 0 & -2.571437 & -1.252449 & 2.409741 \\
\hline 5 & 1 & 0 & -3.320873 & -2.013974 & 2.655092 \\
\hline 6 & 6 & 0 & -1.603250 & -1.533436 & 1.450162 \\
\hline 7 & 1 & 0 & -1.613717 & -2.509293 & 0.956105 \\
\hline 8 & 6 & 0 & -2.590402 & -0.018855 & 3.056579 \\
\hline 9 & 1 & 0 & -3.357819 & 0.203019 & 3.806745 \\
\hline 10 & 6 & 0 & -1.615822 & 0.924814 & 2.744098 \\
\hline 11 & 1 & 0 & -1.608798 & 1.897837 & 3.249005 \\
\hline 12 & 6 & 0 & -0.646307 & 0.644939 & 1.785002 \\
\hline 13 & 1 & 0 & 0.092945 & 1.414025 & 1.550007 \\
\hline 14 & 6 & 0 & -0.626504 & -0.584020 & 1.109700 \\
\hline 15 & 6 & 0 & -0.448097 & -0.031685 & -1.533322 \\
\hline 16 & 6 & 0 & -1.870705 & -0.313862 & -1.471232 \\
\hline 17 & 6 & 0 & -2.785266 & 0.678583 & -1.108137 \\
\hline 18 & 6 & 0 & -1.003863 & 2.349835 & -1.157262 \\
\hline 19 & 6 & 0 & -0.097230 & 1.379831 & -1.504618 \\
\hline 20 & 1 & 0 & 0.942662 & 1.654801 & -1.719515 \\
\hline 21 & 6 & 0 & -2.362684 & 1.993759 & -0.938669 \\
\hline 22 & 1 & 0 & -3.103586 & 2.745859 & -0.642022 \\
\hline 23 & 6 & 0 & 2.571643 & -1.413704 & -0.294363 \\
\hline 24 & 6 & 0 & 1.758182 & -0.355165 & 0.114478 \\
\hline 25 & 6 & 0 & 2.383555 & 0.839829 & 0.475409 \\
\hline 26 & 1 & 0 & 1.803362 & 1.720532 & 0.772864 \\
\hline 27 & 6 & 0 & 3.781190 & 0.948593 & 0.442145 \\
\hline 28 & 6 & 0 & 4.546049 & -0.154861 & 0.042874 \\
\hline 29 & 1 & 0 & 5.639726 & -0.067941 & 0.026288 \\
\hline 30 & 6 & 0 & 3.949968 & -1.358024 & -0.337103 \\
\hline 31 & 1 & 0 & 4.534440 & -2.227279 & -0.653585 \\
\hline 32 & 6 & 0 & -0.594560 & 3.782389 & -1.000550 \\
\hline 33 & 1 & 0 & -1.221681 & 4.457185 & -1.613920 \\
\hline 34 & 1 & 0 & 0.460458 & 3.940147 & -1.288256 \\
\hline 35 & 1 & 0 & -0.711274 & 4.112371 & 0.051529 \\
\hline 36 & 6 & 0 & 0.351903 & -0.824276 & 0.004556 \\
\hline 37 & 6 & 0 & 0.523657 & -2.233194 & -0.484315 \\
\hline 38 & 8 & 0 & -4.081192 & 0.354296 & -0.941958 \\
\hline 39 & 1 & 0 & -4.151114 & -0.615320 & -1.033861 \\
\hline 40 & 6 & 0 & 4.443157 & 2.247124 & 0.800122 \\
\hline 41 & 1 & 0 & 5.477782 & 2.092062 & 1.154897 \\
\hline 42 & 1 & 0 & 4.498428 & 2.928627 & -0.071820 \\
\hline 43 & 1 & 0 & 3.889773 & 2.782077 & 1.593149 \\
\hline 44 & 8 & 0 & 1.836708 & -2.517976 & -0.647808 \\
\hline 45 & 1 & 0 & 0.091887 & -0.621595 & -2.296561 \\
\hline
\end{tabular}

Cartesian coordinates (in Å) and energy of TS-C(6)-B

$\mathrm{E}(\mathrm{UM} 06)=-1148.97962330 \mathrm{au}$

\begin{tabular}{cccccc}
$\begin{array}{c}\text { Center } \\
\text { number }\end{array}$ & $\begin{array}{c}\text { Atomic } \\
\text { number }\end{array}$ & $\begin{array}{c}\text { Atomic } \\
\text { type }\end{array}$ & \multicolumn{3}{c}{ Coordinates (Angstroms) } \\
\hline 1 & 8 & 0 & 1.169677 & -1.743225 & -1.671937 \\
2 & 1 & 0 & 1.980094 & -1.265110 & -1.414236 \\
3 & 8 & 0 & 1.331475 & 3.120638 & -0.107332 \\
4 & 6 & 0 & 4.484859 & 0.228645 & -0.375846 \\
5 & 1 & 0 & 5.280272 & 0.677321 & -0.982074 \\
6 & 6 & 0 & 3.203546 & 0.773206 & -0.417381 \\
7 & 1 & 0 & 3.009526 & 1.659479 & -1.030928 \\
8 & 6 & 0 & 4.759894 & -0.873158 & 0.433302 \\
9 & 1 & 0 & 5.767735 & -1.302637 & 0.459914 \\
10 & 6 & 0 & 3.742850 & -1.413637 & 1.219127 \\
11 & 1 & 0 & 3.949142 & -2.267073 & 1.875312 \\
12 & 6 & 0 & 2.460696 & -0.873297 & 1.180447 \\
13 & 1 & 0 & 1.678074 & -1.298863 & 1.817307 \\
14 & 6 & 0 & 2.160458 & 0.220453 & 0.350858 \\
15 & 6 & 0 & 0.217785 & 0.517807 & -1.573235 \\
16 & 6 & 0 & 0.099468 & -0.907791 & -1.719856
\end{tabular}




\begin{tabular}{|c|c|c|c|c|c|}
\hline 17 & 6 & 0 & -1.148335 & -1.523841 & -1.756153 \\
\hline 18 & 6 & 0 & -2.232941 & 0.666136 & -1.839721 \\
\hline 19 & 6 & 0 & -1.000722 & 1.274923 & -1.787307 \\
\hline 20 & 1 & 0 & -0.924806 & 2.368788 & -1.839963 \\
\hline 21 & 6 & 0 & -2.308004 & -0.750196 & -1.808461 \\
\hline 22 & 1 & 0 & -3.275994 & -1.265739 & -1.830395 \\
\hline 23 & 6 & 0 & -1.080653 & 1.376504 & 1.462341 \\
\hline 24 & 6 & 0 & -0.335173 & 0.261822 & 1.068978 \\
\hline 25 & 6 & 0 & -0.838803 & -1.010957 & 1.320045 \\
\hline 26 & 1 & 0 & -0.311170 & -1.905806 & 0.962173 \\
\hline 27 & 6 & 0 & -2.065892 & -1.161205 & 1.983030 \\
\hline 28 & 6 & 0 & -2.764043 & -0.015901 & 2.383136 \\
\hline 29 & 1 & 0 & -3.724470 & -0.134938 & 2.900678 \\
\hline 30 & 6 & 0 & -2.284885 & 1.274471 & 2.128613 \\
\hline 31 & 1 & 0 & -2.841337 & 2.169620 & 2.423298 \\
\hline 32 & 6 & 0 & -3.498869 & 1.460642 & -1.938172 \\
\hline 33 & 1 & 0 & -4.099561 & 1.161477 & -2.818267 \\
\hline 34 & 1 & 0 & -3.295401 & 2.543159 & -2.013206 \\
\hline 35 & 1 & 0 & -4.134749 & 1.297987 & -1.045847 \\
\hline 36 & 6 & 0 & 0.783478 & 0.769080 & 0.263549 \\
\hline 37 & 6 & 0 & 0.647553 & 2.261393 & 0.367505 \\
\hline 38 & 8 & 0 & -1.238777 & -2.871831 & -1.731735 \\
\hline 39 & 1 & 0 & -0.334715 & -3.234242 & -1.689205 \\
\hline 40 & 6 & 0 & -2.618680 & -2.536776 & 2.212544 \\
\hline 41 & 1 & 0 & -3.641470 & -2.502800 & 2.627619 \\
\hline 42 & 1 & 0 & -2.656135 & -3.110354 & 1.266031 \\
\hline 43 & 1 & 0 & -1.993435 & -3.119274 & 2.916742 \\
\hline 44 & 8 & 0 & -0.495622 & 2.550912 & 1.069341 \\
\hline 45 & 1 & 0 & 1.132848 & 0.967101 & -1.995706 \\
\hline
\end{tabular}

Cartesian coordinates (in Å) and energy of TS-C(6)-C $\mathrm{E}(\mathrm{UM} 06)=-1148.97176708 \mathrm{au}$

\begin{tabular}{|c|c|c|c|c|c|}
\hline \multirow{2}{*}{$\begin{array}{l}\text { Center } \\
\text { number }\end{array}$} & \multirow{2}{*}{$\begin{array}{l}\text { Atomic } \\
\text { number }\end{array}$} & \multirow{2}{*}{$\begin{array}{l}\text { Atomic } \\
\text { type }\end{array}$} & \multicolumn{3}{|c|}{ Coordinates (Angstroms) } \\
\hline & & & $\mathrm{X}$ & $\mathrm{Y}$ & $\mathrm{Z}$ \\
\hline 1 & 8 & 0 & 1.374166 & -1.887966 & -1.370245 \\
\hline 2 & 1 & 0 & 1.802225 & -1.012938 & -1.303874 \\
\hline 3 & 8 & 0 & -1.521213 & -0.470588 & 2.066817 \\
\hline 4 & 6 & 0 & -1.286535 & 3.808829 & -1.264461 \\
\hline 5 & 1 & 0 & -1.062247 & 4.386310 & -2.168816 \\
\hline 6 & 6 & 0 & -0.606495 & 2.618743 & -1.022354 \\
\hline 7 & 1 & 0 & 0.129811 & 2.278734 & -1.760067 \\
\hline 8 & 6 & 0 & -2.249619 & 4.260096 & -0.364483 \\
\hline 9 & 1 & 0 & -2.786764 & 5.196951 & -0.551373 \\
\hline 10 & 6 & 0 & -2.525960 & 3.502878 & 0.771590 \\
\hline 11 & 1 & 0 & -3.284765 & 3.842420 & 1.486249 \\
\hline 12 & 6 & 0 & -1.848490 & 2.310077 & 1.013348 \\
\hline 13 & 1 & 0 & -2.092100 & 1.721636 & 1.902464 \\
\hline 14 & 6 & 0 & -0.866242 & 1.850142 & 0.123724 \\
\hline 15 & 6 & 0 & -0.684125 & -0.575010 & -1.053719 \\
\hline 16 & 6 & 0 & 0.059744 & -1.816380 & -1.029841 \\
\hline 17 & 6 & 0 & -0.534608 & -3.007718 & -0.624195 \\
\hline 18 & 6 & 0 & -2.706270 & -1.882503 & -0.517649 \\
\hline 19 & 6 & 0 & -2.123377 & -0.707373 & -0.926676 \\
\hline 20 & 1 & 0 & -2.737243 & 0.191849 & -1.065770 \\
\hline 21 & 6 & 0 & -1.902632 & -3.039469 & -0.349093 \\
\hline 22 & 1 & 0 & -2.342088 & -3.988301 & -0.019405 \\
\hline 23 & 6 & 0 & 1.750890 & -0.349570 & 1.329231 \\
\hline 24 & 6 & 0 & 1.355091 & 0.555109 & 0.336317 \\
\hline 25 & 6 & 0 & 2.335766 & 1.136693 & -0.471271 \\
\hline 26 & 1 & 0 & 2.072261 & 1.864145 & -1.248490 \\
\hline 27 & 6 & 0 & 3.687388 & 0.803708 & -0.286150 \\
\hline 28 & 6 & 0 & 4.030927 & -0.119799 & 0.711581 \\
\hline 29 & 1 & 0 & 5.086926 & -0.386061 & 0.845958 \\
\hline 30 & 6 & 0 & 3.069693 & -0.704673 & 1.538981 \\
\hline 31 & 1 & 0 & 3.334546 & -1.423599 & 2.320453 \\
\hline 32 & 6 & 0 & -4.168803 & -1.958368 & -0.207571 \\
\hline 33 & 1 & 0 & -4.625076 & -2.888179 & -0.595167 \\
\hline 34 & 1 & 0 & -4.719555 & -1.098502 & -0.627862 \\
\hline
\end{tabular}




$\begin{array}{lllrrr}35 & 1 & 0 & -4.324929 & -1.947487 & 0.889209 \\ 36 & 6 & 0 & -0.121734 & 0.578313 & 0.353371 \\ 37 & 6 & 0 & -0.463191 & -0.247101 & 1.559973 \\ 38 & 8 & 0 & 0.195853 & -4.137857 & -0.540353 \\ 39 & 1 & 0 & 1.124314 & -3.908743 & -0.729384 \\ 40 & 6 & 0 & 4.750667 & 1.455974 & -1.119607 \\ 41 & 1 & 0 & 5.620893 & 0.790559 & -1.263532 \\ 42 & 1 & 0 & 4.371261 & 1.741424 & -2.117387 \\ 43 & 1 & 0 & 5.126693 & 2.381309 & -0.640338 \\ 44 & 8 & 0 & 0.692352 & -0.840948 & 2.028687 \\ 45 & 1 & 0 & -0.335946 & 0.121749 & -1.838378\end{array}$

Cartesian coordinates (in Å) and energy of TS-C(6)-D $\mathrm{E}(\mathrm{UM} 06)=-1148.97269563 \mathrm{au}$

\begin{tabular}{|c|c|c|c|c|c|}
\hline \multirow{2}{*}{$\begin{array}{l}\text { Center } \\
\text { number }\end{array}$} & \multirow{2}{*}{$\begin{array}{l}\text { Atomic } \\
\text { number }\end{array}$} & \multirow{2}{*}{$\begin{array}{l}\text { Atomic } \\
\text { type }\end{array}$} & \multicolumn{3}{|c|}{ Coordinates (Angstroms) } \\
\hline & & & $\mathrm{X}$ & $\mathrm{Y}$ & $\mathrm{Z}$ \\
\hline 1 & 8 & 0 & 2.893836 & 0.018508 & -1.018762 \\
\hline 2 & 1 & 0 & 2.521001 & -0.882272 & -0.994339 \\
\hline 3 & 8 & 0 & 1.220569 & 0.685073 & 2.090569 \\
\hline 4 & 6 & 0 & 2.567930 & -3.228350 & 1.034649 \\
\hline 5 & 1 & 0 & 3.305610 & -3.482187 & 1.804541 \\
\hline 6 & 6 & 0 & 1.778244 & -2.093507 & 1.196567 \\
\hline 7 & 1 & 0 & 1.915996 & -1.459330 & 2.076718 \\
\hline 8 & 6 & 0 & 2.433890 & -4.033876 & -0.095269 \\
\hline 9 & 1 & 0 & 3.062662 & -4.922366 & -0.221534 \\
\hline 10 & 6 & 0 & 1.492686 & -3.694345 & -1.065159 \\
\hline 11 & 1 & 0 & 1.375294 & -4.314604 & -1.961233 \\
\hline 12 & 6 & 0 & 0.696988 & -2.561757 & -0.904266 \\
\hline 13 & 1 & 0 & -0.023920 & -2.308755 & -1.690456 \\
\hline 14 & 6 & 0 & 0.820950 & -1.737961 & 0.230543 \\
\hline 15 & 6 & 0 & 0.520481 & 0.652753 & -1.046077 \\
\hline 16 & 6 & 0 & 1.922410 & 0.954028 & -0.877279 \\
\hline 17 & 6 & 0 & 2.348228 & 2.221512 & -0.481920 \\
\hline 18 & 6 & 0 & 0.055349 & 3.054006 & -0.683596 \\
\hline 19 & 6 & 0 & -0.368483 & 1.806148 & -1.067921 \\
\hline 20 & 1 & 0 & -1.420221 & 1.641904 & -1.336462 \\
\hline 21 & 6 & 0 & 1.425686 & 3.259820 & -0.370459 \\
\hline 22 & 1 & 0 & 1.788785 & 4.242108 & -0.045663 \\
\hline 23 & 6 & 0 & -1.995667 & 0.326433 & 1.189097 \\
\hline 24 & 6 & 0 & -1.491674 & -0.585764 & 0.256580 \\
\hline 25 & 6 & 0 & -2.383427 & -1.241067 & -0.588373 \\
\hline 26 & 1 & 0 & -2.033165 & -1.965415 & -1.333978 \\
\hline 27 & 6 & 0 & -3.759313 & -0.981313 & -0.496648 \\
\hline 28 & 6 & 0 & -4.214791 & -0.048015 & 0.443756 \\
\hline 29 & 1 & 0 & -5.290240 & 0.161987 & 0.505095 \\
\hline 30 & 6 & 0 & -3.340110 & 0.618390 & 1.305687 \\
\hline 31 & 1 & 0 & -3.691347 & 1.345226 & 2.044717 \\
\hline 32 & 6 & 0 & -0.893432 & 4.209339 & -0.590792 \\
\hline 33 & 1 & 0 & -0.957011 & 4.581952 & 0.449940 \\
\hline 34 & 1 & 0 & -1.912262 & 3.925650 & -0.909043 \\
\hline 35 & 1 & 0 & -0.561064 & 5.060444 & -1.215493 \\
\hline 36 & 6 & 0 & -0.019704 & -0.515505 & 0.363038 \\
\hline 37 & 6 & 0 & 0.198305 & 0.373190 & 1.552825 \\
\hline 38 & 8 & 0 & 3.652879 & 2.450953 & -0.240899 \\
\hline 39 & 1 & 0 & 4.119222 & 1.595293 & -0.270543 \\
\hline 40 & 6 & 0 & -4.728509 & -1.714407 & -1.376921 \\
\hline 41 & 1 & 0 & -5.651904 & -1.130211 & -1.540680 \\
\hline 42 & 1 & 0 & -4.290869 & -1.940968 & -2.366314 \\
\hline 43 & 1 & 0 & -5.032064 & -2.681522 & -0.929414 \\
\hline 44 & 8 & 0 & -1.009135 & 0.894886 & 1.943327 \\
\hline 45 & 1 & 0 & 0.314083 & -0.100799 & -1.828113 \\
\hline
\end{tabular}

Cartesian coordinates (in Å) and energy of TS-C(6)-E $\mathrm{E}(\mathrm{UM} 06)=-1148.97205351 \mathrm{au}$

Center Atomic Atomic Coordinates (Angstroms)




\begin{tabular}{|c|c|c|c|c|c|}
\hline number & number & type & $\mathrm{X}$ & $\mathrm{Y}$ & $\mathrm{Z}$ \\
\hline 1 & 8 & 0 & 0.718238 & 2.039094 & -1.410106 \\
\hline 2 & 1 & 0 & 1.501890 & 1.466598 & -1.316974 \\
\hline 3 & 8 & 0 & -0.356955 & -3.152561 & -0.562411 \\
\hline 4 & 6 & 0 & -1.752091 & 1.039522 & 2.631139 \\
\hline 5 & 1 & 0 & -1.829258 & 2.068942 & 2.999645 \\
\hline 6 & 6 & 0 & -0.802359 & 0.727570 & 1.661990 \\
\hline 7 & 1 & 0 & -0.177766 & 1.535999 & 1.267789 \\
\hline 8 & 6 & 0 & -2.607021 & 0.053333 & 3.116252 \\
\hline 9 & 1 & 0 & -3.357852 & 0.297041 & 3.876837 \\
\hline 10 & 6 & 0 & -2.497818 & -1.245757 & 2.624372 \\
\hline 11 & 1 & 0 & -3.162913 & -2.032908 & 2.998654 \\
\hline 12 & 6 & 0 & -1.550603 & -1.558650 & 1.652686 \\
\hline 13 & 1 & 0 & -1.493131 & -2.578722 & 1.263017 \\
\hline 14 & 6 & 0 & -0.682665 & -0.576093 & 1.154260 \\
\hline 15 & 6 & 0 & -0.441310 & -0.133888 & -1.488814 \\
\hline 16 & 6 & 0 & -0.431888 & 1.311917 & -1.368157 \\
\hline 17 & 6 & 0 & -1.591718 & 2.031535 & -1.113126 \\
\hline 18 & 6 & 0 & -2.916872 & -0.001108 & -1.404943 \\
\hline 19 & 6 & 0 & -1.772783 & -0.717209 & -1.654665 \\
\hline 20 & 1 & 0 & -1.840752 & -1.784163 & -1.893802 \\
\hline 21 & 6 & 0 & -2.829122 & 1.385474 & -1.111023 \\
\hline 22 & 1 & 0 & -3.725904 & 1.978104 & -0.894527 \\
\hline 23 & 6 & 0 & 2.480906 & -1.372051 & -0.495754 \\
\hline 24 & 6 & 0 & 1.705574 & -0.404640 & 0.153333 \\
\hline 25 & 6 & 0 & 2.360661 & 0.700381 & 0.703770 \\
\hline 26 & 1 & 0 & 1.807421 & 1.467561 & 1.256237 \\
\hline 27 & 6 & 0 & 3.748227 & 0.847938 & 0.558740 \\
\hline 28 & 6 & 0 & 4.469501 & -0.128253 & -0.142069 \\
\hline 29 & 1 & 0 & 5.552888 & -0.004270 & -0.264065 \\
\hline 30 & 6 & 0 & 3.847807 & -1.261133 & -0.669188 \\
\hline 31 & 1 & 0 & 4.407204 & -2.045417 & -1.188521 \\
\hline 32 & 6 & 0 & -4.265351 & -0.652920 & -1.428476 \\
\hline 33 & 1 & 0 & -4.696828 & -0.688883 & -0.408520 \\
\hline 34 & 1 & 0 & -4.209829 & -1.689539 & -1.804033 \\
\hline 35 & 1 & 0 & -4.979597 & -0.092294 & -2.060880 \\
\hline 36 & 6 & 0 & 0.291172 & -0.860315 & 0.058767 \\
\hline 37 & 6 & 0 & 0.445933 & -2.276362 & -0.437731 \\
\hline 38 & 8 & 0 & -1.524986 & 3.358455 & -0.868603 \\
\hline 39 & 1 & 0 & -0.590224 & 3.628046 & -0.923767 \\
\hline 40 & 6 & 0 & 4.451544 & 2.018710 & 1.180142 \\
\hline 41 & 1 & 0 & 5.378016 & 2.273340 & 0.634830 \\
\hline 42 & 1 & 0 & 3.809902 & 2.918184 & 1.199686 \\
\hline 43 & 1 & 0 & 4.740372 & 1.805574 & 2.228200 \\
\hline 44 & 8 & 0 & 1.737583 & -2.443491 & -0.890217 \\
\hline 45 & 1 & 0 & 0.316978 & -0.503170 & -2.208138 \\
\hline
\end{tabular}

Cartesian coordinates (in $\AA$ ) and energy of TS-C(6)-F $\mathrm{E}(\mathrm{UM} 06)=-1148.97835064 \mathrm{au}$

\begin{tabular}{cccccc}
$\begin{array}{c}\text { Center } \\
\text { number }\end{array}$ & $\begin{array}{c}\text { Atomic } \\
\text { number }\end{array}$ & $\begin{array}{c}\text { Atomic } \\
\text { type }\end{array}$ & \multicolumn{3}{c}{ Coordinates (Angstroms) } \\
\hline 1 & 8 & 0 & -0.964273 & -3.064882 & 0.385266 \\
2 & 1 & 0 & -0.093486 & -3.303111 & 0.006535 \\
3 & 8 & 0 & 1.376998 & -2.639345 & -1.257194 \\
4 & 6 & 0 & 3.821281 & 1.977305 & -0.039366 \\
5 & 1 & 0 & 4.028705 & 3.049991 & -0.129509 \\
6 & 6 & 0 & 2.543023 & 1.500556 & -0.320103 \\
7 & 1 & 0 & 1.772152 & 2.204438 & -0.649341 \\
8 & 6 & 0 & 4.832055 & 1.098686 & 0.342946 \\
9 & 1 & 0 & 5.837773 & 1.473599 & 0.564822 \\
10 & 6 & 0 & 4.553488 & -0.264640 & 0.428331 \\
11 & 1 & 0 & 5.343023 & -0.970301 & 0.711862 \\
12 & 6 & 0 & 3.277050 & -0.743527 & 0.149917 \\
13 & 1 & 0 & 3.084443 & -1.820617 & 0.192582 \\
14 & 6 & 0 & 2.243870 & 0.134128 & -0.221500 \\
15 & 6 & 0 & 0.257494 & -1.081188 & 1.174611 \\
16 & 6 & 0 & -0.952485 & -1.844995 & 0.974976 \\
17 & 6 & 0 & -2.201052 & -1.317494 & 1.306265
\end{tabular}




\begin{tabular}{|c|c|c|c|c|c|}
\hline 18 & 6 & 0 & -1.099707 & 0.583134 & 2.385566 \\
\hline 19 & 6 & 0 & 0.129083 & 0.076627 & 2.039487 \\
\hline 20 & 1 & 0 & 1.048396 & 0.597193 & 2.339478 \\
\hline 21 & 6 & 0 & -2.278471 & -0.105333 & 1.989998 \\
\hline 22 & 1 & 0 & -3.271873 & 0.285725 & 2.241920 \\
\hline 23 & 6 & 0 & -0.893813 & -0.262903 & -1.966224 \\
\hline 24 & 6 & 0 & -0.215748 & 0.481812 & -1.000375 \\
\hline 25 & 6 & 0 & -0.735294 & 1.719894 & -0.626120 \\
\hline 26 & 1 & 0 & -0.249900 & 2.319202 & 0.154310 \\
\hline 27 & 6 & 0 & -1.912186 & 2.195060 & -1.221159 \\
\hline 28 & 6 & 0 & -2.545422 & 1.415789 & -2.198253 \\
\hline 29 & 1 & 0 & -3.463715 & 1.793881 & -2.665330 \\
\hline 30 & 6 & 0 & -2.046888 & 0.170781 & -2.588942 \\
\hline 31 & 1 & 0 & -2.543644 & -0.445400 & -3.344511 \\
\hline 32 & 6 & 0 & -1.233190 & 1.857113 & 3.162715 \\
\hline 33 & 1 & 0 & -1.714147 & 2.645116 & 2.548655 \\
\hline 34 & 1 & 0 & -0.250698 & 2.239666 & 3.491547 \\
\hline 35 & 1 & 0 & -1.867026 & 1.723256 & 4.059903 \\
\hline 36 & 6 & 0 & 0.871061 & -0.375529 & -0.492980 \\
\hline 37 & 6 & 0 & 0.762524 & -1.604587 & -1.340356 \\
\hline 38 & 8 & 0 & -3.324790 & -1.997716 & 1.007128 \\
\hline 39 & 1 & 0 & -3.071738 & -2.793241 & 0.503158 \\
\hline 40 & 6 & 0 & -2.513784 & 3.494810 & -0.773619 \\
\hline 41 & 1 & 0 & -3.073616 & 3.989993 & -1.587668 \\
\hline 42 & 1 & 0 & -3.227798 & 3.340366 & 0.060537 \\
\hline 43 & 1 & 0 & -1.742488 & 4.200011 & -0.413866 \\
\hline 44 & 8 & 0 & -0.297492 & -1.480237 & -2.186861 \\
\hline 45 & 1 & 0 & 1.154781 & -1.705348 & 1.333330 \\
\hline
\end{tabular}

Cartesian coordinates (in Å) and energy of INT-II-C(6)-A

$\mathrm{E}(\mathrm{UM} 06)=-1148.98257064 \mathrm{au}$

\begin{tabular}{|c|c|c|c|c|c|}
\hline \multirow{2}{*}{$\begin{array}{l}\text { Center } \\
\text { number }\end{array}$} & \multirow{2}{*}{$\begin{array}{l}\text { Atomic } \\
\text { number }\end{array}$} & \multirow{2}{*}{$\begin{array}{l}\text { Atomic } \\
\text { type }\end{array}$} & \multicolumn{3}{|c|}{ Coordinates (Angstroms) } \\
\hline & & & $\mathrm{X}$ & $\mathrm{Y}$ & $\mathrm{Z}$ \\
\hline 1 & 8 & 0 & -2.582673 & -1.049189 & -1.538321 \\
\hline 2 & 1 & 0 & -1.838633 & -1.602958 & -1.880120 \\
\hline 3 & 8 & 0 & -0.245344 & -2.172139 & -2.294356 \\
\hline 4 & 6 & 0 & -0.717796 & -3.192397 & 2.027437 \\
\hline 5 & 1 & 0 & -0.658809 & -4.281018 & 2.139946 \\
\hline 6 & 6 & 0 & -0.243176 & -2.596010 & 0.859773 \\
\hline 7 & 1 & 0 & 0.178373 & -3.236283 & 0.075038 \\
\hline 8 & 6 & 0 & -1.265763 & -2.411372 & 3.040720 \\
\hline 9 & 1 & 0 & -1.642378 & -2.880253 & 3.956984 \\
\hline 10 & 6 & 0 & -1.334006 & -1.028253 & 2.879783 \\
\hline 11 & 1 & 0 & -1.767550 & -0.402577 & 3.668280 \\
\hline 12 & 6 & 0 & -0.857786 & -0.431928 & 1.715742 \\
\hline 13 & 1 & 0 & -0.918847 & 0.657054 & 1.599407 \\
\hline 14 & 6 & 0 & -0.308508 & -1.210021 & 0.689342 \\
\hline 15 & 6 & 0 & -0.739919 & 0.574049 & -1.173998 \\
\hline 16 & 6 & 0 & -2.167008 & 0.146593 & -1.073431 \\
\hline 17 & 6 & 0 & -3.150708 & 0.948848 & -0.507990 \\
\hline 18 & 6 & 0 & -1.521069 & 2.727824 & -0.114019 \\
\hline 19 & 6 & 0 & -0.525560 & 1.956609 & -0.643786 \\
\hline 20 & 1 & 0 & 0.480417 & 2.378420 & -0.761290 \\
\hline 21 & 6 & 0 & -2.850491 & 2.222217 & -0.018138 \\
\hline 22 & 1 & 0 & -3.658072 & 2.822184 & 0.416644 \\
\hline 23 & 6 & 0 & 2.521932 & -0.651000 & -1.146102 \\
\hline 24 & 6 & 0 & 1.652837 & -0.012987 & -0.270327 \\
\hline 25 & 6 & 0 & 2.173521 & 0.854839 & 0.680892 \\
\hline 26 & 1 & 0 & 1.512953 & 1.342713 & 1.408992 \\
\hline 27 & 6 & 0 & 3.553760 & 1.103127 & 0.717254 \\
\hline 28 & 6 & 0 & 4.387926 & 0.448610 & -0.199535 \\
\hline 29 & 1 & 0 & 5.468196 & 0.637503 & -0.162399 \\
\hline 30 & 6 & 0 & 3.887825 & -0.448275 & -1.145737 \\
\hline 31 & 1 & 0 & 4.537208 & -0.978740 & -1.848624 \\
\hline 32 & 6 & 0 & -1.252677 & 4.123402 & 0.363870 \\
\hline 33 & 1 & 0 & -1.852643 & 4.860934 & -0.203501 \\
\hline 34 & 1 & 0 & -0.188088 & 4.397432 & 0.257246 \\
\hline 35 & 1 & 0 & -1.532381 & 4.242613 & 1.428597 \\
\hline
\end{tabular}




$\begin{array}{lllrrr}36 & 6 & 0 & 0.261478 & -0.525909 & -0.542565 \\ 37 & 6 & 0 & 0.550084 & -1.516073 & -1.673386 \\ 38 & 8 & 0 & -4.419450 & 0.491945 & -0.440355 \\ 39 & 1 & 0 & -4.427549 & -0.410813 & -0.809506 \\ 40 & 6 & 0 & 4.120160 & 2.070958 & 1.713834 \\ 41 & 1 & 0 & 5.187478 & 1.870788 & 1.915865 \\ 42 & 1 & 0 & 4.047812 & 3.115126 & 1.350708 \\ 43 & 1 & 0 & 3.577517 & 2.026642 & 2.675560 \\ 44 & 8 & 0 & 1.860329 & -1.528695 & -1.983009 \\ 45 & 1 & 0 & -0.458250 & 0.588748 & -2.257308\end{array}$

Cartesian coordinates (in Å) and energy of INT-II-C(6)-B $\mathrm{E}(\mathrm{UM} 06)=-1148.98408575 \mathrm{au}$

\begin{tabular}{|c|c|c|c|c|c|}
\hline \multirow{2}{*}{$\begin{array}{l}\text { Center } \\
\text { number }\end{array}$} & \multirow{2}{*}{$\begin{array}{l}\text { Atomic } \\
\text { number }\end{array}$} & \multirow{2}{*}{$\begin{array}{l}\text { Atomic } \\
\text { type }\end{array}$} & \multicolumn{3}{|c|}{ Coordinates (Angstroms) } \\
\hline & & & $\mathrm{X}$ & $\mathrm{Y}$ & $\mathrm{Z}$ \\
\hline 1 & 8 & 0 & -0.980392 & 2.470324 & 0.227413 \\
\hline 2 & 1 & 0 & -1.810611 & 1.978864 & 0.088342 \\
\hline 3 & 8 & 0 & -1.408330 & -1.885596 & -2.411905 \\
\hline 4 & 6 & 0 & -4.450163 & 0.360813 & -0.429596 \\
\hline 5 & 1 & 0 & -5.237412 & 0.601826 & -1.153032 \\
\hline 6 & 6 & 0 & -3.164342 & 0.075703 & -0.884973 \\
\hline 7 & 1 & 0 & -2.963527 & 0.068101 & -1.961873 \\
\hline 8 & 6 & 0 & -4.737092 & 0.332673 & 0.934036 \\
\hline 9 & 1 & 0 & -5.747581 & 0.561485 & 1.291133 \\
\hline 10 & 6 & 0 & -3.730196 & 0.000974 & 1.837765 \\
\hline 11 & 1 & 0 & -3.944939 & -0.037433 & 2.911870 \\
\hline 12 & 6 & 0 & -2.443631 & -0.284365 & 1.384606 \\
\hline 13 & 1 & 0 & -1.664437 & -0.550028 & 2.107227 \\
\hline 14 & 6 & 0 & -2.136229 & -0.239711 & 0.018699 \\
\hline 15 & 6 & 0 & -0.165414 & 0.741681 & -1.351986 \\
\hline 16 & 6 & 0 & 0.052822 & 1.902789 & -0.456692 \\
\hline 17 & 6 & 0 & 1.315282 & 2.370539 & -0.139672 \\
\hline 18 & 6 & 0 & 2.312590 & 0.851339 & -1.780834 \\
\hline 19 & 6 & 0 & 1.075947 & 0.361252 & -2.091851 \\
\hline 20 & 1 & 0 & 0.969528 & -0.381079 & -2.894646 \\
\hline 21 & 6 & 0 & 2.450522 & 1.851031 & -0.773769 \\
\hline 22 & 1 & 0 & 3.434421 & 2.243512 & -0.491659 \\
\hline 23 & 6 & 0 & 0.848017 & -2.122546 & 0.074288 \\
\hline 24 & 6 & 0 & 0.302852 & -0.917515 & 0.504976 \\
\hline 25 & 6 & 0 & 0.865491 & -0.263735 & 1.591598 \\
\hline 26 & 1 & 0 & 0.470303 & 0.703335 & 1.931605 \\
\hline 27 & 6 & 0 & 1.978130 & -0.819701 & 2.241574 \\
\hline 28 & 6 & 0 & 2.490671 & -2.037161 & 1.779582 \\
\hline 29 & 1 & 0 & 3.364208 & -2.469490 & 2.283947 \\
\hline 30 & 6 & 0 & 1.934447 & -2.713653 & 0.688715 \\
\hline 31 & 1 & 0 & 2.345751 & -3.657724 & 0.318655 \\
\hline 32 & 6 & 0 & 3.545259 & 0.360452 & -2.477157 \\
\hline 33 & 1 & 0 & 4.131800 & 1.197222 & -2.902061 \\
\hline 34 & 1 & 0 & 3.301871 & -0.340275 & -3.294889 \\
\hline 35 & 1 & 0 & 4.209769 & -0.168462 & -1.765835 \\
\hline 36 & 6 & 0 & -0.733468 & -0.509321 & -0.494133 \\
\hline 37 & 6 & 0 & -0.735771 & -1.716401 & -1.442024 \\
\hline 38 & 8 & 0 & 1.463830 & 3.322945 & 0.810750 \\
\hline 39 & 1 & 0 & 0.577192 & 3.565585 & 1.134307 \\
\hline 40 & 6 & 0 & 2.605782 & -0.087917 & 3.390602 \\
\hline 41 & 1 & 0 & 3.566811 & -0.542551 & 3.689346 \\
\hline 42 & 1 & 0 & 2.797504 & 0.970300 & 3.127449 \\
\hline 43 & 1 & 0 & 1.949588 & -0.083554 & 4.282719 \\
\hline 44 & 8 & 0 & 0.217703 & -2.607957 & -1.047240 \\
\hline 45 & 1 & 0 & -0.981752 & 0.959318 & -2.077284 \\
\hline
\end{tabular}

Cartesian coordinates (in $\AA$ ) and energy of INT-II-C(6)-C $\mathrm{E}(\mathrm{UM} 06)=-1148.97562914$

\begin{tabular}{lllrc} 
Center & Atomic & Atomic & \multicolumn{2}{c}{ Coordinates (Angstroms) } \\
number & number & $X$ & $Y$ & $Z$
\end{tabular}




\begin{tabular}{|c|c|c|c|c|c|}
\hline 1 & 8 & 0 & 0.256114 & -2.491060 & -1.080919 \\
\hline 2 & 1 & 0 & 1.058178 & -1.947125 & -0.984652 \\
\hline 3 & 8 & 0 & -1.467324 & 0.166079 & 2.136539 \\
\hline 4 & 6 & 0 & 0.356211 & 3.891888 & -1.357793 \\
\hline 5 & 1 & 0 & 0.621620 & 4.309272 & -2.336013 \\
\hline 6 & 6 & 0 & 0.350451 & 2.511892 & -1.177604 \\
\hline 7 & 1 & 0 & 0.603918 & 1.869683 & -2.030259 \\
\hline 8 & 6 & 0 & 0.022962 & 4.737094 & -0.300835 \\
\hline 9 & 1 & 0 & 0.026133 & 5.824250 & -0.439306 \\
\hline 10 & 6 & 0 & -0.318735 & 4.184879 & 0.929911 \\
\hline 11 & 1 & 0 & -0.588784 & 4.836166 & 1.769163 \\
\hline 12 & 6 & 0 & -0.327460 & 2.801910 & 1.108933 \\
\hline 13 & 1 & 0 & -0.619463 & 2.394812 & 2.081802 \\
\hline 14 & 6 & 0 & 0.011602 & 1.943339 & 0.058405 \\
\hline 15 & 6 & 0 & -0.884190 & -0.285776 & -0.881589 \\
\hline 16 & 6 & 0 & -0.867303 & -1.772458 & -0.782885 \\
\hline 17 & 6 & 0 & -1.992735 & -2.511504 & -0.470753 \\
\hline 18 & 6 & 0 & -3.365981 & -0.482158 & -0.517691 \\
\hline 19 & 6 & 0 & -2.267800 & 0.269731 & -0.826189 \\
\hline 20 & 1 & 0 & -2.375143 & 1.350613 & -0.984024 \\
\hline 21 & 6 & 0 & -3.236299 & -1.883661 & -0.305138 \\
\hline 22 & 1 & 0 & -4.102850 & -2.503017 & -0.046767 \\
\hline 23 & 6 & 0 & 1.611514 & -0.905553 & 1.334011 \\
\hline 24 & 6 & 0 & 1.450263 & -0.123447 & 0.192015 \\
\hline 25 & 6 & 0 & 2.484414 & -0.047878 & -0.735535 \\
\hline 26 & 1 & 0 & 2.385456 & 0.566744 & -1.639627 \\
\hline 27 & 6 & 0 & 3.671972 & -0.766497 & -0.519372 \\
\hline 28 & 6 & 0 & 3.784879 & -1.551963 & 0.636051 \\
\hline 29 & 1 & 0 & 4.708269 & -2.121834 & 0.799472 \\
\hline 30 & 6 & 0 & 2.761806 & -1.631632 & 1.583539 \\
\hline 31 & 1 & 0 & 2.849782 & -2.247015 & 2.484044 \\
\hline 32 & 6 & 0 & -4.722393 & 0.139436 & -0.382279 \\
\hline 33 & 1 & 0 & -5.471637 & -0.383924 & -1.006486 \\
\hline 34 & 1 & 0 & -4.714987 & 1.204865 & -0.671702 \\
\hline 35 & 1 & 0 & -5.075943 & 0.077847 & 0.665119 \\
\hline 36 & 6 & 0 & 0.052883 & 0.433637 & 0.218596 \\
\hline 37 & 6 & 0 & -0.438837 & -0.058316 & 1.584629 \\
\hline 38 & 8 & 0 & -1.916847 & -3.856546 & -0.370877 \\
\hline 39 & 1 & 0 & -0.986531 & -4.112314 & -0.508100 \\
\hline 40 & 6 & 0 & 4.808600 & -0.666621 & -1.492760 \\
\hline 41 & 1 & 0 & 5.435142 & -1.576338 & -1.482214 \\
\hline 42 & 1 & 0 & 4.449074 & -0.512505 & -2.526210 \\
\hline 43 & 1 & 0 & 5.471649 & 0.187281 & -1.250947 \\
\hline 44 & 8 & 0 & 0.508389 & -0.890097 & 2.137201 \\
\hline 45 & 1 & 0 & -0.391476 & -0.002516 & -1.842856 \\
\hline
\end{tabular}

Cartesian coordinates (in $\AA$ ) and energy of INT-II-C(6)-D $\mathrm{E}(\mathrm{UM} 06)=-1148.97725671 \mathrm{au}$

\begin{tabular}{cccccc}
$\begin{array}{c}\text { Center } \\
\text { number }\end{array}$ & $\begin{array}{c}\text { Atomic } \\
\text { number }\end{array}$ & $\begin{array}{c}\text { Atomic } \\
\text { type }\end{array}$ & X & \multicolumn{3}{c}{ Coordinates (Angstroms) } \\
\hline 1 & 8 & 0 & -2.655681 & 1.258444 & -0.875304 \\
2 & 1 & 0 & -1.957870 & 1.886231 & -0.612744 \\
3 & 8 & 0 & -1.319637 & -0.066132 & 2.153165 \\
4 & 6 & 0 & -0.374564 & 4.024549 & 1.072433 \\
5 & 1 & 0 & -0.677445 & 4.633003 & 1.932122 \\
6 & 6 & 0 & -0.325752 & 2.636740 & 1.202208 \\
7 & 1 & 0 & -0.607439 & 2.181985 & 2.156817 \\
8 & 6 & 0 & -0.050449 & 4.634606 & -0.136177 \\
9 & 1 & 0 & -0.095305 & 5.724857 & -0.236097 \\
10 & 6 & 0 & 0.327200 & 3.843575 & -1.220061 \\
11 & 1 & 0 & 0.580341 & 4.307459 & -2.180140 \\
12 & 6 & 0 & 0.379624 & 2.458539 & -1.091016 \\
13 & 1 & 0 & 0.664246 & 1.858139 & -1.963255 \\
14 & 6 & 0 & 0.056249 & 1.828494 & 0.122446 \\
15 & 6 & 0 & -0.815554 & -0.409536 & -0.857617 \\
16 & 6 & 0 & -2.246467 & -0.030324 & -0.709290 \\
17 & 6 & 0 & -3.245623 & -0.952384 & -0.443965
\end{tabular}




\begin{tabular}{lllrrr}
18 & 6 & 0 & -1.622098 & -2.780026 & -0.565976 \\
19 & 6 & 0 & -0.618007 & -1.892877 & -0.830252 \\
20 & 1 & 0 & 0.397099 & -2.264215 & -1.023670 \\
21 & 6 & 0 & -2.952567 & -2.316895 & -0.348128 \\
22 & 1 & 0 & -3.766804 & -3.013643 & -0.118812 \\
23 & 6 & 0 & 1.792129 & -0.965646 & 1.258175 \\
24 & 6 & 0 & 1.550128 & -0.201380 & 0.120447 \\
25 & 6 & 0 & 2.537301 & -0.100678 & -0.849854 \\
26 & 1 & 0 & 2.374907 & 0.483547 & -1.764720 \\
27 & 6 & 0 & 3.764830 & -0.758333 & -0.672676 \\
28 & 6 & 0 & 3.964321 & -1.517140 & 0.487585 \\
29 & 1 & 0 & 4.921130 & -2.037449 & 0.622562 \\
30 & 6 & 0 & 2.982375 & -1.633846 & 1.473554 \\
31 & 1 & 0 & 3.133103 & -2.230987 & 2.378080 \\
32 & 6 & 0 & -1.364272 & -4.255437 & -0.512505 \\
33 & 1 & 0 & -1.630398 & -4.667133 & 0.480045 \\
34 & 1 & 0 & -0.303870 & -4.494419 & -0.706467 \\
35 & 1 & 0 & -1.980498 & -4.796231 & -1.256417 \\
36 & 6 & 0 & 0.138029 & 0.312844 & 0.217531 \\
37 & 6 & 0 & -0.283174 & -0.227557 & 1.587684 \\
38 & 8 & 0 & -4.520578 & -0.537958 & -0.296116 \\
39 & 1 & 0 & -4.518162 & 0.436410 & -0.336870 \\
40 & 6 & 0 & 4.850862 & -0.624836 & -1.699092 \\
41 & 1 & 0 & 5.539339 & -1.488557 & -1.678646 \\
42 & 1 & 0 & 4.438070 & -0.544373 & -2.721238 \\
43 & 1 & 0 & 5.462945 & 0.282398 & -1.526119 \\
44 & 8 & 0 & 0.720999 & -0.990140 & 2.112349 \\
45 & 1 & 0 & -0.441331 & -0.005446 & -1.829919 \\
------------------------------------------------------------------------ \\
\hline
\end{tabular}

Cartesian coordinates (in $\AA$ ) and energy of INT-II-C(6)-E $\mathrm{E}(\mathrm{UM} 06)=-1148.97310974 \mathrm{au}$

\begin{tabular}{|c|c|c|c|c|c|}
\hline \multirow{2}{*}{$\begin{array}{l}\text { Center } \\
\text { number }\end{array}$} & \multirow{2}{*}{$\begin{array}{l}\text { Atomic } \\
\text { number }\end{array}$} & \multirow{2}{*}{$\begin{array}{l}\text { Atomic } \\
\text { type }\end{array}$} & \multicolumn{3}{|c|}{ Coordinates (Angstroms) } \\
\hline & & & $\mathrm{X}$ & $\mathrm{Y}$ & Z \\
\hline 1 & 8 & 0 & 0.740470 & 2.490788 & -0.692473 \\
\hline 2 & 1 & 0 & 1.495074 & 1.875098 & -0.720308 \\
\hline 3 & 8 & 0 & -0.354372 & -2.499330 & -2.059457 \\
\hline 4 & 6 & 0 & -1.698688 & -0.561418 & 2.848212 \\
\hline 5 & 1 & 0 & -1.885395 & 0.179282 & 3.634261 \\
\hline 6 & 6 & 0 & -0.892862 & -0.223232 & 1.764805 \\
\hline 7 & 1 & 0 & -0.481026 & 0.790625 & 1.711707 \\
\hline 8 & 6 & 0 & -2.267585 & -1.830571 & 2.929674 \\
\hline 9 & 1 & 0 & -2.902166 & -2.099460 & 3.781990 \\
\hline 10 & 6 & 0 & -2.021369 & -2.752647 & 1.916498 \\
\hline 11 & 1 & 0 & -2.461735 & -3.755344 & 1.965608 \\
\hline 12 & 6 & 0 & -1.220184 & -2.411945 & 0.827840 \\
\hline 13 & 1 & 0 & -1.062415 & -3.147173 & 0.033475 \\
\hline 14 & 6 & 0 & -0.643596 & -1.140387 & 0.734280 \\
\hline 15 & 6 & 0 & -0.549265 & 0.462211 & -1.310346 \\
\hline 16 & 6 & 0 & -0.446005 & 1.813703 & -0.708013 \\
\hline 17 & 6 & 0 & -1.543797 & 2.506933 & -0.231210 \\
\hline 18 & 6 & 0 & -3.031806 & 0.738991 & -1.041035 \\
\hline 19 & 6 & 0 & -1.964368 & 0.049895 & -1.543677 \\
\hline 20 & 1 & 0 & -2.123245 & -0.896524 & -2.073704 \\
\hline 21 & 6 & 0 & -2.831956 & 1.969747 & -0.351435 \\
\hline 22 & 1 & 0 & -3.676220 & 2.538892 & 0.054618 \\
\hline 23 & 6 & 0 & 2.456167 & -0.983368 & -1.036948 \\
\hline 24 & 6 & 0 & 1.632232 & -0.348875 & -0.108736 \\
\hline 25 & 6 & 0 & 2.223415 & 0.434943 & 0.880247 \\
\hline 26 & 1 & 0 & 1.612681 & 0.929609 & 1.644800 \\
\hline 27 & 6 & 0 & 3.615688 & 0.611410 & 0.903482 \\
\hline 28 & 6 & 0 & 4.396251 & -0.017438 & -0.075829 \\
\hline 29 & 1 & 0 & 5.483683 & 0.128270 & -0.062289 \\
\hline 30 & 6 & 0 & 3.830948 & -0.834550 & -1.055613 \\
\hline 31 & 1 & 0 & 4.437513 & -1.349678 & -1.806725 \\
\hline 32 & 6 & 0 & -4.429790 & 0.219884 & -1.190051 \\
\hline 33 & 1 & 0 & -4.846527 & -0.066371 & -0.204342 \\
\hline 34 & 1 & 0 & -4.466544 & -0.670963 & -1.840993 \\
\hline 35 & 1 & 0 & -5.105473 & 0.987102 & -1.614015 \\
\hline 36 & 6 & 0 & 0.208989 & -0.717601 & -0.444118 \\
\hline
\end{tabular}




$\begin{array}{rrrrrr}37 & 6 & 0 & 0.434388 & -1.793048 & -1.512203 \\ 38 & 8 & 0 & -1.383912 & 3.725584 & 0.333372 \\ 39 & 1 & 0 & -0.436197 & 3.948884 & 0.294428 \\ 40 & 6 & 0 & 4.254305 & 1.433217 & 1.983812 \\ 41 & 1 & 0 & 5.233229 & 1.834157 & 1.666015 \\ 42 & 1 & 0 & 3.616500 & 2.286003 & 2.279024 \\ 43 & 1 & 0 & 4.430067 & 0.831000 & 2.896785 \\ 44 & 8 & 0 & 1.753977 & -1.789593 & -1.887445 \\ 45 & 1 & 0 & 0.022229 & 0.481869 & -2.270117\end{array}$

Cartesian coordinates (in ̊) and energy of INT-II-C(6)-F $\mathrm{E}(\mathrm{UM} 06)=-1148.98353948$ au

\begin{tabular}{|c|c|c|c|c|c|}
\hline \multirow{2}{*}{$\begin{array}{l}\text { Center } \\
\text { number }\end{array}$} & \multirow{2}{*}{$\begin{array}{l}\text { Atomic } \\
\text { number }\end{array}$} & \multirow{2}{*}{$\begin{array}{l}\text { Atomic } \\
\text { type }\end{array}$} & \multicolumn{3}{|c|}{ Coordinates (Angstroms) } \\
\hline & & & $\mathrm{X}$ & $\mathrm{Y}$ & $\mathrm{Z}$ \\
\hline 1 & 8 & 0 & -1.394121 & -1.064940 & -2.503203 \\
\hline 2 & 1 & 0 & -0.559070 & -1.177289 & -2.996946 \\
\hline 3 & 8 & 0 & 1.323339 & -0.117533 & -2.949696 \\
\hline 4 & 6 & 0 & 3.684687 & 0.623377 & 1.764724 \\
\hline 5 & 1 & 0 & 3.906544 & 1.185799 & 2.679122 \\
\hline 6 & 6 & 0 & 2.412549 & 0.706891 & 1.199856 \\
\hline 7 & 1 & 0 & 1.655636 & 1.338912 & 1.676508 \\
\hline 8 & 6 & 0 & 4.667553 & -0.163828 & 1.171637 \\
\hline 9 & 1 & 0 & 5.667611 & -0.228694 & 1.615477 \\
\hline 10 & 6 & 0 & 4.370019 & -0.862104 & 0.002409 \\
\hline 11 & 1 & 0 & 5.137466 & -1.476156 & -0.482853 \\
\hline 12 & 6 & 0 & 3.100231 & -0.780307 & -0.561741 \\
\hline 13 & 1 & 0 & 2.891151 & -1.314337 & -1.495279 \\
\hline 14 & 6 & 0 & 2.099918 & 0.000577 & 0.034604 \\
\hline 15 & 6 & 0 & 0.059281 & -1.423318 & -0.537102 \\
\hline 16 & 6 & 0 & -1.268202 & -1.463671 & -1.204337 \\
\hline 17 & 6 & 0 & -2.435582 & -1.796368 & -0.545813 \\
\hline 18 & 6 & 0 & -1.157460 & -2.291572 & 1.488312 \\
\hline 19 & 6 & 0 & 0.006815 & -1.945897 & 0.860886 \\
\hline 20 & 1 & 0 & 0.963108 & -2.034503 & 1.393503 \\
\hline 21 & 6 & 0 & -2.400748 & -2.197988 & 0.798449 \\
\hline 22 & 1 & 0 & -3.344234 & -2.467472 & 1.287239 \\
\hline 23 & 6 & 0 & -0.403058 & 2.036332 & -1.039648 \\
\hline 24 & 6 & 0 & -0.210463 & 1.095995 & -0.032952 \\
\hline 25 & 6 & 0 & -0.854057 & 1.269781 & 1.184864 \\
\hline 26 & 1 & 0 & -0.727273 & 0.530875 & 1.987513 \\
\hline 27 & 6 & 0 & -1.675599 & 2.390178 & 1.387852 \\
\hline 28 & 6 & 0 & -1.820583 & 3.321722 & 0.352650 \\
\hline 29 & 1 & 0 & -2.456108 & 4.201304 & 0.515779 \\
\hline 30 & 6 & 0 & -1.189528 & 3.160316 & -0.883354 \\
\hline 31 & 1 & 0 & -1.312227 & 3.879085 & -1.699143 \\
\hline 32 & 6 & 0 & -1.164324 & -2.769822 & 2.909341 \\
\hline 33 & 1 & 0 & -1.748968 & -2.086171 & 3.556120 \\
\hline 34 & 1 & 0 & -0.143164 & -2.839157 & 3.323683 \\
\hline 35 & 1 & 0 & -1.637242 & -3.766874 & 2.995809 \\
\hline 36 & 6 & 0 & 0.700319 & 0.033205 & -0.576959 \\
\hline 37 & 6 & 0 & 0.830280 & 0.485565 & -2.037699 \\
\hline 38 & 8 & 0 & -3.620499 & -1.763854 & -1.192176 \\
\hline 39 & 1 & 0 & -3.455569 & -1.469449 & -2.106495 \\
\hline 40 & 6 & 0 & -2.403271 & 2.559683 & 2.688782 \\
\hline 41 & 1 & 0 & -2.791485 & 3.586514 & 2.809686 \\
\hline 42 & 1 & 0 & -3.267789 & 1.870627 & 2.757651 \\
\hline 43 & 1 & 0 & -1.749643 & 2.340116 & 3.553319 \\
\hline 44 & 8 & 0 & 0.253798 & 1.699942 & -2.202828 \\
\hline 45 & 1 & 0 & 0.778451 & -2.034529 & -1.136031 \\
\hline
\end{tabular}

Cartesian coordinates (in $\AA$ ) and energy of INT-I-C(5)-A $\mathrm{E}(\mathrm{UM} 06)=-1149.01426152 \mathrm{au}$

\begin{tabular}{llccc} 
Center & Atomic & Atomic & & \multicolumn{2}{c}{ Coordinates (Angstroms) } \\
number & number & type & $X$ & $Y$
\end{tabular}




\begin{tabular}{|c|c|c|c|c|c|}
\hline 1 & 8 & 0 & -1.266165 & 3.148912 & -0.767838 \\
\hline 2 & 8 & 0 & -0.817665 & -2.448456 & 1.531716 \\
\hline 3 & 1 & 0 & -1.738418 & -2.329093 & 1.240723 \\
\hline 4 & 8 & 0 & 1.846328 & -2.323530 & 1.548382 \\
\hline 5 & 1 & 0 & 1.205149 & -3.047578 & 1.423663 \\
\hline 6 & 6 & 0 & -0.504773 & 2.245026 & -0.974509 \\
\hline 7 & 6 & 0 & -0.715599 & 0.785334 & -1.074356 \\
\hline 8 & 6 & 0 & 0.574364 & 0.218618 & -1.285434 \\
\hline 9 & 6 & 0 & 1.113468 & -1.081993 & -1.374956 \\
\hline & 1 & 0 & 0.480184 & -1.968742 & -1.261139 \\
\hline & 6 & 0 & 2.482617 & -1.266868 & -1.544523 \\
\hline & 6 & 0 & 3.324077 & -0.140464 & -1.630343 \\
\hline & 1 & 0 & 4.401165 & -0.296971 & -1.772027 \\
\hline & 6 & 0 & 2.833698 & 1.162267 & -1.520127 \\
\hline & 1 & 0 & 3.488276 & 2.038235 & -1.565132 \\
\hline & 6 & 0 & 1.473956 & 1.310255 & -1.339248 \\
\hline 7 & 6 & 0 & -0.312380 & 1.140895 & 2.237660 \\
\hline & 6 & 0 & -0.976735 & -0.074338 & 2.062367 \\
\hline & 1 & 0 & -2.073926 & -0.124540 & 2.091931 \\
\hline & 6 & 0 & -0.256455 & -1.230618 & 1.802772 \\
\hline & 6 & 0 & 1.145580 & -1.191064 & 1.783682 \\
\hline & 6 & 0 & 1.800608 & 0.020311 & 1.974487 \\
\hline & 6 & 0 & 1.083667 & 1.205648 & 2.179705 \\
\hline & 6 & 0 & -2.006847 & 0.157259 & -0.918319 \\
\hline & 6 & 0 & -3.117165 & 0.880549 & -0.424301 \\
\hline & 1 & 0 & -3.001042 & 1.946855 & -0.211557 \\
\hline & 6 & 0 & -4.330972 & 0.249157 & -0.185336 \\
\hline & 1 & 0 & -5.171297 & 0.831832 & 0.209461 \\
\hline & 6 & 0 & -4.486703 & -1.115147 & -0.437454 \\
\hline 0 & 1 & 0 & -5.445643 & -1.609384 & -0.244105 \\
\hline & 6 & 0 & -3.414119 & -1.838712 & -0.964079 \\
\hline 2 & 1 & 0 & -3.533685 & -2.901602 & -1.206546 \\
\hline 3 & 6 & 0 & -2.197046 & -1.212104 & -1.207273 \\
\hline 4 & 1 & 0 & -1.385921 & -1.790298 & -1.657536 \\
\hline 5 & 6 & 0 & 3.069193 & -2.646285 & -1.564134 \\
\hline 6 & 1 & 0 & 2.337494 & -3.395143 & -1.918010 \\
\hline 7 & 1 & 0 & 3.380715 & -2.946242 & -0.544428 \\
\hline 8 & 1 & 0 & 3.958615 & -2.705131 & -2.217623 \\
\hline 9 & 1 & 0 & 2.897063 & 0.024793 & 1.928378 \\
\hline 0 & 6 & 0 & 1.797388 & 2.520739 & 2.280383 \\
\hline 1 & 1 & 0 & 1.872771 & 3.006993 & 1.285905 \\
\hline 2 & 1 & 0 & 1.261943 & 3.225829 & 2.941906 \\
\hline 3 & 1 & 0 & 2.825464 & 2.400013 & 2.667457 \\
\hline 4 & 8 & 0 & 0.844383 & 2.497134 & -1.169737 \\
\hline 5 & 1 & 0 & -0.892881 & 2.056907 & 2.408521 \\
\hline
\end{tabular}

Cartesian coordinates (in Å) and energy of INT-I-C(5)-B

$\mathrm{E}(\mathrm{UM} 06)=-1149.01241739 \mathrm{au}$

\begin{tabular}{rrrrrr}
$\begin{array}{c}\text { Center } \\
\text { number }\end{array}$ & $\begin{array}{c}\text { Atomic } \\
\text { number }\end{array}$ & $\begin{array}{c}\text { Atomic } \\
\text { type }\end{array}$ & \multicolumn{3}{c}{ Coordinates (Angstroms) } \\
\hline 1 & 8 & 0 & -1.510667 & -1.493327 & 2.416610 \\
2 & 8 & 0 & 3.311705 & -0.513903 & -1.588635 \\
3 & 1 & 0 & 3.340645 & 0.312240 & -2.095992 \\
4 & 8 & 0 & 2.795767 & -2.355013 & 0.272701 \\
5 & 1 & 0 & 3.591746 & -1.843834 & 0.038462 \\
6 & 6 & 0 & -0.736798 & -0.692660 & 1.966347 \\
7 & 6 & 0 & -0.925361 & 0.415742 & 1.011392 \\
8 & 6 & 0 & 0.358588 & 1.000380 & 0.835468 \\
9 & 6 & 0 & 0.885770 & 2.084990 & 0.103116 \\
10 & 1 & 0 & 0.230979 & 2.726353 & -0.497518 \\
11 & 6 & 0 & 2.247394 & 2.366162 & 0.155848 \\
12 & 6 & 0 & 3.087964 & 1.568305 & 0.961999 \\
13 & 1 & 0 & 4.160465 & 1.801947 & 0.996223 \\
14 & 6 & 0 & 2.597788 & 0.507519 & 1.721887 \\
15 & 1 & 0 & 3.242513 & -0.110752 & 2.354206 \\
16 & 6 & 0 & 1.243048 & 0.246960 & 1.641972 \\
17 & 6 & 0 & -0.297663 & -1.059042 & -2.154035 \\
18 & 6 & 0 & 0.988975 & -0.545209 & -2.330116
\end{tabular}




$\begin{array}{rlrrrc}19 & 1 & 0 & 1.191015 & 0.209129 & -3.103559 \\ 20 & 6 & 0 & 2.027503 & -0.974312 & -1.515628 \\ 21 & 6 & 0 & 1.789594 & -1.943675 & -0.527312 \\ 22 & 6 & 0 & 0.506166 & -2.449219 & -0.361973 \\ 23 & 6 & 0 & -0.557720 & -2.010797 & -1.164392 \\ 24 & 6 & 0 & -2.196439 & 0.723964 & 0.398542 \\ 25 & 6 & 0 & -3.408464 & 0.294763 & 0.982526 \\ 26 & 1 & 0 & -3.378205 & -0.265175 & 1.921220 \\ 27 & 6 & 0 & -4.627111 & 0.575201 & 0.377119 \\ 28 & 1 & 0 & -5.555217 & 0.237185 & 0.852229 \\ 29 & 6 & 0 & -4.675626 & 1.279053 & -0.826542 \\ 30 & 1 & 0 & -5.639099 & 1.493521 & -1.303145 \\ 31 & 6 & 0 & -3.486581 & 1.703922 & -1.422971 \\ 32 & 1 & 0 & -3.512963 & 2.245523 & -2.375712 \\ 33 & 6 & 0 & -2.265753 & 1.433491 & -0.819774 \\ 34 & 1 & 0 & -1.341469 & 1.730948 & -1.324675 \\ 35 & 6 & 0 & 2.838119 & 3.500449 & -0.630078 \\ 36 & 1 & 0 & 2.062252 & 4.073361 & -1.167819 \\ 37 & 1 & 0 & 3.567711 & 3.139291 & -1.382639 \\ 38 & 1 & 0 & 3.385617 & 4.207732 & 0.021537 \\ 39 & 1 & 0 & 0.342300 & -3.180632 & 0.439925 \\ 40 & 6 & 0 & -1.937478 & -2.555077 & -0.945398 \\ 41 & 1 & 0 & -2.694275 & -1.980727 & -1.511228 \\ 42 & 1 & 0 & -2.012398 & -3.613742 & -1.262917 \\ 43 & 1 & 0 & -2.214528 & -2.519533 & 0.126607 \\ 44 & 8 & 0 & 0.604980 & -0.735630 & 2.314252 \\ 45 & 1 & 0 & -1.116859 & -0.705309 & -2.792608 \\ ---------------------------------------------------------------------------\end{array}$

Cartesian coordinates (in Å) and energy of INT-I-C(5)-C $\mathrm{E}(\mathrm{UM} 06)=-1149.02064416 \mathrm{au}$

\begin{tabular}{|c|c|c|c|c|c|}
\hline \multirow{2}{*}{$\begin{array}{l}\text { Center } \\
\text { number }\end{array}$} & \multirow{2}{*}{$\begin{array}{l}\text { Atomic } \\
\text { number }\end{array}$} & \multirow{2}{*}{$\begin{array}{l}\text { Atomic } \\
\text { type }\end{array}$} & \multicolumn{3}{|c|}{ Coordinates (Angstroms) } \\
\hline & & & $\mathrm{X}$ & $\mathrm{Y}$ & $\mathrm{Z}$ \\
\hline 1 & 8 & 0 & 1.291251 & 0.816742 & 2.693446 \\
\hline 2 & 8 & 0 & 2.167678 & 2.276467 & 0.481833 \\
\hline 3 & 1 & 0 & 2.082675 & 1.999164 & 1.422135 \\
\hline 4 & 8 & 0 & 2.124194 & 1.178798 & -1.947943 \\
\hline 5 & 1 & 0 & 2.815574 & 1.299812 & -1.266187 \\
\hline 6 & 6 & 0 & 0.400940 & 0.218359 & 2.130747 \\
\hline 7 & 6 & 0 & 0.426760 & -0.711595 & 0.992962 \\
\hline 8 & 6 & 0 & -0.938882 & -1.001244 & 0.704784 \\
\hline 9 & 6 & 0 & -1.631277 & -1.797484 & -0.231179 \\
\hline 10 & 1 & 0 & -1.089352 & -2.408536 & -0.960695 \\
\hline 11 & 6 & 0 & -3.022154 & -1.832930 & -0.224725 \\
\hline 12 & 6 & 0 & -3.729079 & -1.077647 & 0.735114 \\
\hline 13 & 1 & 0 & -4.826284 & -1.114886 & 0.730357 \\
\hline 14 & 6 & 0 & -3.078184 & -0.302993 & 1.694418 \\
\hline 15 & 1 & 0 & -3.622947 & 0.271692 & 2.449787 \\
\hline 16 & 6 & 0 & -1.697642 & -0.284486 & 1.655895 \\
\hline 17 & 6 & 0 & -1.441036 & 2.421915 & -0.269502 \\
\hline 18 & 6 & 0 & -0.242886 & 2.609772 & 0.420660 \\
\hline 19 & 1 & 0 & -0.230841 & 3.069256 & 1.416620 \\
\hline 20 & 6 & 0 & 0.958103 & 2.180958 & -0.133182 \\
\hline 21 & 6 & 0 & 0.962777 & 1.595634 & -1.414911 \\
\hline 22 & 6 & 0 & -0.234758 & 1.427345 & -2.101331 \\
\hline 23 & 6 & 0 & -1.453135 & 1.821802 & -1.532739 \\
\hline 24 & 6 & 0 & 1.644076 & -1.151111 & 0.352063 \\
\hline 25 & 6 & 0 & 2.892146 & -1.035460 & 1.005806 \\
\hline 26 & 1 & 0 & 2.930430 & -0.619328 & 2.016198 \\
\hline 27 & 6 & 0 & 4.061205 & -1.456083 & 0.385512 \\
\hline 28 & 1 & 0 & 5.016123 & -1.362968 & 0.915491 \\
\hline 29 & 6 & 0 & 4.025539 & -2.000762 & -0.899450 \\
\hline 30 & 1 & 0 & 4.951137 & -2.329641 & -1.385665 \\
\hline 31 & 6 & 0 & 2.804128 & -2.113929 & -1.564051 \\
\hline 32 & 1 & 0 & 2.767364 & -2.514858 & -2.583084 \\
\hline 33 & 6 & 0 & 1.632697 & -1.693048 & -0.950981 \\
\hline 34 & 1 & 0 & 0.696130 & -1.729461 & -1.514964 \\
\hline 35 & 6 & 0 & -3.782220 & -2.658767 & -1.220200 \\
\hline 36 & 1 & 0 & -4.411321 & -2.024810 & -1.875010 \\
\hline
\end{tabular}




$\begin{array}{lllrrr}37 & 1 & 0 & -4.462537 & -3.373908 & -0.719861 \\ 38 & 1 & 0 & -3.104919 & -3.239722 & -1.870449 \\ 39 & 1 & 0 & -0.200045 & 0.953865 & -3.091215 \\ 40 & 6 & 0 & -2.737529 & 1.575307 & -2.265936 \\ 41 & 1 & 0 & -3.603190 & 1.991346 & -1.719999 \\ 42 & 1 & 0 & -2.726123 & 2.025759 & -3.277022 \\ 43 & 1 & 0 & -2.918915 & 0.489837 & -2.399548 \\ 44 & 8 & 0 & -0.907480 & 0.412032 & 2.511085 \\ 45 & 1 & 0 & -2.385641 & 2.743771 & 0.187086\end{array}$

Cartesian coordinates (in $\AA$ ) and energy of INT-I-C(5)-D $\mathrm{E}(\mathrm{UM} 06)=-1149.01837599$ au

\begin{tabular}{|c|c|c|c|c|c|}
\hline \multirow{2}{*}{$\begin{array}{l}\text { Center } \\
\text { number }\end{array}$} & \multirow{2}{*}{$\begin{array}{l}\text { Atomic } \\
\text { number }\end{array}$} & \multirow{2}{*}{$\begin{array}{l}\text { Atomic } \\
\text { type }\end{array}$} & \multicolumn{3}{|c|}{ Coordinates (Angstroms) } \\
\hline & & & $\mathrm{X}$ & $\mathrm{Y}$ & $\mathrm{Z}$ \\
\hline 1 & 8 & 0 & 1.105544 & -2.057629 & -1.871311 \\
\hline 2 & 8 & 0 & -0.493419 & -3.553572 & -0.127952 \\
\hline 3 & 1 & 0 & 0.199331 & -3.395392 & -0.807363 \\
\hline 4 & 8 & 0 & -2.835963 & -2.324058 & 0.172724 \\
\hline 5 & 1 & 0 & -2.490706 & -3.047902 & -0.389095 \\
\hline 6 & 6 & 0 & 0.458685 & -1.056808 & -1.663594 \\
\hline 7 & 6 & 0 & 0.831130 & 0.228294 & -1.058895 \\
\hline 8 & 6 & 0 & -0.369752 & 0.985923 & -0.991087 \\
\hline 9 & 6 & 0 & -0.723364 & 2.275653 & -0.543343 \\
\hline 10 & 1 & 0 & 0.037237 & 2.963172 & -0.156529 \\
\hline 11 & 6 & 0 & -2.048189 & 2.693970 & -0.610062 \\
\hline 12 & 6 & 0 & -3.021678 & 1.823248 & -1.146972 \\
\hline 13 & 1 & 0 & -4.063662 & 2.165517 & -1.193521 \\
\hline 14 & 6 & 0 & -2.702726 & 0.553309 & -1.628403 \\
\hline 15 & 1 & 0 & -3.456561 & -0.123570 & -2.040367 \\
\hline 16 & 6 & 0 & -1.381080 & 0.167763 & -1.541722 \\
\hline 17 & 6 & 0 & 0.429679 & -0.895993 & 2.265323 \\
\hline 18 & 6 & 0 & 0.564744 & -2.004430 & 1.425765 \\
\hline 19 & 1 & 0 & 1.538957 & -2.487230 & 1.276456 \\
\hline 20 & 6 & 0 & -0.535522 & -2.496159 & 0.735448 \\
\hline 21 & 6 & 0 & -1.788044 & -1.872923 & 0.887062 \\
\hline 22 & 6 & 0 & -1.915527 & -0.772610 & 1.727597 \\
\hline 23 & 6 & 0 & -0.808613 & -0.267881 & 2.423843 \\
\hline 24 & 6 & 0 & 2.158064 & 0.523094 & -0.573338 \\
\hline 25 & 6 & 0 & 3.284883 & -0.153874 & -1.087420 \\
\hline 26 & 1 & 0 & 3.144798 & -0.908485 & -1.866896 \\
\hline 27 & 6 & 0 & 4.559852 & 0.135027 & -0.617628 \\
\hline 28 & 1 & 0 & 5.422873 & -0.394278 & -1.037397 \\
\hline 29 & 6 & 0 & 4.746124 & 1.091317 & 0.381383 \\
\hline 30 & 1 & 0 & 5.753613 & 1.313891 & 0.751173 \\
\hline 31 & 6 & 0 & 3.640167 & 1.755677 & 0.915475 \\
\hline 32 & 1 & 0 & 3.775509 & 2.492165 & 1.715869 \\
\hline 33 & 6 & 0 & 2.363741 & 1.477139 & 0.446080 \\
\hline 34 & 1 & 0 & 1.501079 & 1.967336 & 0.909504 \\
\hline 35 & 6 & 0 & -2.459513 & 4.045721 & -0.106377 \\
\hline 36 & 1 & 0 & -1.585214 & 4.690806 & 0.090606 \\
\hline 37 & 1 & 0 & -3.031391 & 3.966238 & 0.839110 \\
\hline 38 & 1 & 0 & -3.110712 & 4.569801 & -0.830567 \\
\hline 39 & 1 & 0 & -2.899673 & -0.292449 & 1.807018 \\
\hline 40 & 6 & 0 & -0.966800 & 0.929304 & 3.314058 \\
\hline 41 & 1 & 0 & 0.012559 & 1.358613 & 3.594649 \\
\hline 42 & 1 & 0 & -1.559568 & 1.724247 & 2.820822 \\
\hline 43 & 1 & 0 & -1.494596 & 0.674372 & 4.254243 \\
\hline 44 & 8 & 0 & -0.888745 & -1.029376 & -1.954984 \\
\hline 45 & 1 & 0 & 1.309227 & -0.508334 & 2.795321 \\
\hline
\end{tabular}

Cartesian coordinates (in $\AA$ ) and energy of INT-I-C(5)-E $\mathrm{E}(\mathrm{UM} 06)=-1149.01271858 \mathrm{au}$

\begin{tabular}{lllcc} 
Center & Atomic & Atomic & \multicolumn{2}{c}{ Coordinates (Angstroms) } \\
number & number & type & $X$ & $Y$
\end{tabular}




\begin{tabular}{|c|c|c|c|c|c|}
\hline 1 & 8 & 0 & -0.851550 & -1.332493 & 2.736787 \\
\hline 2 & 8 & 0 & -2.133748 & -0.380114 & -2.205267 \\
\hline 3 & 1 & 0 & -2.171848 & 0.568290 & -1.978994 \\
\hline 4 & 8 & 0 & -2.146107 & -2.393956 & -0.456852 \\
\hline 5 & 1 & 0 & -2.828909 & -1.817964 & -0.856399 \\
\hline 6 & 6 & 0 & -0.102176 & -0.634592 & 2.114259 \\
\hline 7 & 6 & 0 & -0.345843 & 0.464412 & 1.153410 \\
\hline 8 & 6 & 0 & 0.943665 & 0.930045 & 0.760612 \\
\hline 9 & 6 & 0 & 1.455566 & 1.900588 & -0.128133 \\
\hline & 1 & 0 & 0.791134 & 2.528231 & -0.730234 \\
\hline & 6 & 0 & 2.828112 & 2.065653 & -0.280894 \\
\hline & 6 & 0 & 3.708390 & 1.249179 & 0.458754 \\
\hline & 1 & 0 & 4.790014 & 1.391039 & 0.334840 \\
\hline & 6 & 0 & 3.244321 & 0.269318 & 1.334524 \\
\hline & 1 & 0 & 3.922518 & -0.373443 & 1.904719 \\
\hline & 6 & 0 & 1.875286 & 0.127710 & 1.460308 \\
\hline & 6 & 0 & 1.486635 & -1.061988 & -1.901170 \\
\hline & 6 & 0 & 0.300492 & -0.465052 & -2.334826 \\
\hline & 1 & 0 & 0.314499 & 0.362978 & -3.055069 \\
\hline & 6 & 0 & -0.919296 & -0.907407 & -1.845475 \\
\hline & 6 & 0 & -0.960638 & -1.974379 & -0.930082 \\
\hline & 6 & 0 & 0.225725 & -2.563774 & -0.502279 \\
\hline & 6 & 0 & 1.462747 & -2.115520 & -0.981607 \\
\hline & 6 & 0 & -1.669996 & 0.892603 & 0.764854 \\
\hline & 6 & 0 & -2.817736 & 0.152568 & 1.135548 \\
\hline & 1 & 0 & -2.694804 & -0.744934 & 1.746695 \\
\hline & 6 & 0 & -4.084729 & 0.544909 & 0.719264 \\
\hline & 1 & 0 & -4.954932 & -0.050046 & 1.020495 \\
\hline & 6 & 0 & -4.258716 & 1.679001 & -0.074100 \\
\hline & 1 & 0 & -5.260252 & 1.981330 & -0.400097 \\
\hline & 6 & 0 & -3.141194 & 2.435288 & -0.434893 \\
\hline & 1 & 0 & -3.262707 & 3.345749 & -1.033649 \\
\hline & 6 & 0 & -1.870263 & 2.051565 & -0.021017 \\
\hline & 1 & 0 & -1.020738 & 2.688110 & -0.280614 \\
\hline & 6 & 0 & 3.385947 & 3.083896 & -1.231018 \\
\hline & 1 & 0 & 2.588950 & 3.696578 & -1.687888 \\
\hline & 1 & 0 & 3.950227 & 2.604039 & -2.054154 \\
\hline & 1 & 0 & 4.087792 & 3.772108 & -0.722706 \\
\hline & 1 & 0 & 0.163427 & -3.373272 & 0.236364 \\
\hline & 6 & 0 & 2.725709 & -2.773045 & -0.510418 \\
\hline & 1 & 0 & 2.731237 & -2.885778 & 0.590891 \\
\hline & 1 & 0 & 2.836030 & -3.789568 & -0.936580 \\
\hline & 1 & 0 & 3.620122 & -2.192291 & -0.800219 \\
\hline & 8 & 0 & 1.270996 & -0.783626 & 2.253872 \\
\hline & 1 & 0 & 2.449469 & -0.695514 & -2.279430 \\
\hline
\end{tabular}

Cartesian coordinates (in $\AA$ ) and energy of INT-I-C(5)-F

$\mathrm{E}(\mathrm{UM} 06)=-1149.01468100 \mathrm{au}$

\begin{tabular}{rrrrrr}
$\begin{array}{c}\text { Center } \\
\text { number }\end{array}$ & $\begin{array}{c}\text { Atomic } \\
\text { number }\end{array}$ & $\begin{array}{c}\text { Atomic } \\
\text { type }\end{array}$ & \multicolumn{3}{c}{ Cordinates (Angstroms) } \\
\hline 1 & 8 & 0 & Y & Z \\
\hline 2 & 8 & 0 & -2.947461 & -2.156861 & 0.363797 \\
3 & 1 & 0 & -2.649693 & -2.795259 & -0.305418 \\
4 & 8 & 0 & -3.275410 & 0.218943 & 1.560011 \\
5 & 1 & 0 & -3.849248 & -0.328054 & 0.991747 \\
6 & 6 & 0 & 0.593062 & -1.483280 & -1.728184 \\
7 & 6 & 0 & 0.930135 & -0.166736 & -1.162358 \\
8 & 6 & 0 & -0.277377 & 0.580502 & -1.155249 \\
9 & 6 & 0 & -0.686388 & 1.819518 & -0.619708 \\
10 & 1 & 0 & 0.022606 & 2.452521 & -0.072857 \\
11 & 6 & 0 & -2.014898 & 2.217105 & -0.719684 \\
12 & 6 & 0 & -2.935985 & 1.383124 & -1.387505 \\
13 & 1 & 0 & -3.978470 & 1.716782 & -1.474054 \\
14 & 6 & 0 & -2.573916 & 0.137073 & -1.900563 \\
15 & 1 & 0 & -3.299174 & -0.528216 & -2.379968 \\
16 & 6 & 0 & -1.257192 & -0.249198 & -1.744973 \\
17 & 6 & 0 & 0.437890 & -1.595593 & 1.723789 \\
18 & 6 & 0 & -0.618783 & -2.233519 & 1.071749
\end{tabular}




$\begin{array}{rrrrrr}19 & 1 & 0 & -0.467514 & -3.206208 & 0.584239 \\ 20 & 6 & 0 & -1.865159 & -1.626808 & 1.007979 \\ 21 & 6 & 0 & -2.069366 & -0.383469 & 1.627345 \\ 22 & 6 & 0 & -1.011204 & 0.238288 & 2.282951 \\ 23 & 6 & 0 & 0.259046 & -0.347269 & 2.327632 \\ 24 & 6 & 0 & 2.254758 & 0.207473 & -0.723765 \\ 25 & 6 & 0 & 3.220204 & -0.768616 & -0.393736 \\ 26 & 1 & 0 & 2.965112 & -1.826634 & -0.505480 \\ 27 & 6 & 0 & 4.473720 & -0.395030 & 0.073506 \\ 28 & 1 & 0 & 5.204074 & -1.169257 & 0.335689 \\ 29 & 6 & 0 & 4.809226 & 0.953592 & 0.207735 \\ 30 & 1 & 0 & 5.800685 & 1.242216 & 0.575181 \\ 31 & 6 & 0 & 3.879011 & 1.931733 & -0.146343 \\ 32 & 1 & 0 & 4.142005 & 2.993333 & -0.072790 \\ 33 & 6 & 0 & 2.619870 & 1.565281 & -0.605278 \\ 34 & 1 & 0 & 1.916287 & 2.342852 & -0.920379 \\ 35 & 6 & 0 & -2.498480 & 3.469271 & -0.054081 \\ 36 & 1 & 0 & -3.023485 & 3.219500 & 0.889874 \\ 37 & 1 & 0 & -3.215085 & 4.023617 & -0.688183 \\ 38 & 1 & 0 & -1.666024 & 4.150599 & 0.196156 \\ 39 & 1 & -1.198107 & 1.219041 & 2.740131 \\ 40 & 6 & 0 & 1.415187 & 0.380331 & 2.945554 \\ 41 & 1 & 0 & 1.917657 & 1.032542 & 2.199764 \\ 42 & 1 & 0 & 1.094978 & 1.025794 & 3.783925 \\ 43 & 1 & 0 & -0.753215 & -1.459142 & -2.092803 \\ 44 & 1 & 0 & 1.423537 & -2.077165 & 1.754470 \\ 45 & 1 & 0 & 0.319614 & 3.323883 \\ --------------------------------------------- \\ & 1 & 0 & & \end{array}$

Cartesian coordinates (in Å) and energy of TS-C(5)-A

$\mathrm{E}(\mathrm{UM} 06)=-1148.97573757 \mathrm{au}$

\begin{tabular}{|c|c|c|c|c|c|}
\hline \multirow{2}{*}{$\begin{array}{l}\text { Center } \\
\text { number }\end{array}$} & \multirow{2}{*}{$\begin{array}{l}\text { Atomic } \\
\text { number }\end{array}$} & \multirow{2}{*}{$\begin{array}{l}\text { Atomic } \\
\text { type }\end{array}$} & \multicolumn{3}{|c|}{ Coordinates (Angstroms) } \\
\hline & & & $\mathrm{X}$ & $\mathrm{Y}$ & $\mathrm{Z}$ \\
\hline 1 & 8 & 0 & 1.960165 & 2.830537 & -0.244511 \\
\hline 2 & 8 & 0 & -1.261563 & -2.996143 & -1.491955 \\
\hline 3 & 1 & 0 & -0.427720 & -3.492166 & -1.457857 \\
\hline 4 & 8 & 0 & -3.397202 & -1.429758 & -1.564029 \\
\hline 5 & 1 & 0 & -3.277637 & -2.396539 & -1.511320 \\
\hline 6 & 6 & 0 & 1.117587 & 2.141470 & 0.253138 \\
\hline 7 & 6 & 0 & 0.951875 & 0.652041 & 0.221689 \\
\hline 8 & 6 & 0 & -0.249696 & 0.418289 & 1.039255 \\
\hline 9 & 6 & 0 & -0.993520 & -0.709805 & 1.376973 \\
\hline 10 & 1 & 0 & -0.643835 & -1.717308 & 1.117452 \\
\hline 11 & 6 & 0 & -2.234308 & -0.571858 & 2.018073 \\
\hline 12 & 6 & 0 & -2.700937 & 0.709764 & 2.327359 \\
\hline 13 & 1 & 0 & -3.671120 & 0.815957 & 2.828898 \\
\hline 14 & 6 & 0 & -1.970422 & 1.859052 & 2.005969 \\
\hline 15 & 1 & 0 & -2.336377 & 2.863956 & 2.238420 \\
\hline 16 & 6 & 0 & -0.764530 & 1.678667 & 1.360066 \\
\hline 17 & 6 & 0 & 0.374978 & 0.347425 & -1.602990 \\
\hline 18 & 6 & 0 & 0.227151 & -1.091827 & -1.667258 \\
\hline 19 & 1 & 0 & 1.124582 & -1.725665 & -1.642503 \\
\hline 20 & 6 & 0 & -1.013847 & -1.661099 & -1.602469 \\
\hline 21 & 6 & 0 & -2.184575 & -0.857750 & -1.658797 \\
\hline 22 & 6 & 0 & -2.058853 & 0.521563 & -1.853242 \\
\hline 23 & 6 & 0 & -0.818835 & 1.135306 & -1.898228 \\
\hline 24 & 6 & 0 & 2.196343 & -0.149520 & 0.359744 \\
\hline 25 & 6 & 0 & 3.340473 & 0.167032 & -0.393234 \\
\hline 26 & 1 & 0 & 3.334348 & 1.052700 & -1.036720 \\
\hline 27 & 6 & 0 & 4.489400 & -0.612947 & -0.306067 \\
\hline 28 & 1 & 0 & 5.369495 & -0.343402 & -0.901678 \\
\hline 29 & 6 & 0 & 4.529207 & -1.725122 & 0.534522 \\
\hline 30 & 1 & 0 & 5.435406 & -2.338316 & 0.600269 \\
\hline 31 & 6 & 0 & 3.409359 & -2.038274 & 1.301301 \\
\hline 32 & 1 & 0 & 3.432195 & -2.895258 & 1.985053 \\
\hline 33 & 6 & 0 & 2.257470 & -1.259021 & 1.215772 \\
\hline 34 & 1 & 0 & 1.399628 & -1.502811 & 1.851063 \\
\hline 35 & 6 & 0 & -3.047188 & -1.796897 & 2.317533 \\
\hline 36 & 1 & 0 & -3.287208 & -2.344624 & 1.385143 \\
\hline
\end{tabular}




$\begin{array}{llllll}37 & 1 & 0 & -4.002797 & -1.545216 & 2.810229 \\ 38 & 1 & 0 & -2.504386 & -2.501711 & 2.976256 \\ 39 & 1 & 0 & -2.978458 & 1.100161 & -2.005800 \\ 40 & 6 & 0 & -0.698629 & 2.592323 & -2.221810 \\ 41 & 1 & 0 & -0.687597 & 3.230419 & -1.316140 \\ 42 & 1 & 0 & 0.239676 & 2.809403 & -2.764617 \\ 43 & 1 & 0 & -1.547986 & 2.928370 & -2.843618 \\ 44 & 8 & 0 & 0.045826 & 2.690791 & 0.922327 \\ 45 & 1 & 0 & 1.300968 & 0.745756 & -2.050985\end{array}$

Cartesian coordinates (in Å) and energy of TS-C(5)-B $\mathrm{E}(\mathrm{UM} 06)=-1148.97135679$ au

\begin{tabular}{|c|c|c|c|c|c|}
\hline \multirow{2}{*}{$\begin{array}{l}\text { Center } \\
\text { number }\end{array}$} & \multirow{2}{*}{$\begin{array}{l}\text { Atomic } \\
\text { number }\end{array}$} & \multirow{2}{*}{$\begin{array}{l}\text { Atomic } \\
\text { type }\end{array}$} & \multicolumn{3}{|c|}{ Coordinates (Angstroms) } \\
\hline & & & $\mathrm{X}$ & $\mathrm{Y}$ & $\mathrm{Z}$ \\
\hline 1 & 8 & 0 & -0.107038 & -1.596703 & 2.051433 \\
\hline 2 & 8 & 0 & 3.946808 & -0.473672 & -0.672954 \\
\hline 3 & 1 & 0 & 3.933041 & 0.494066 & -0.754624 \\
\hline 4 & 8 & 0 & 3.627569 & -3.018150 & 0.028375 \\
\hline 5 & 1 & 0 & 4.390043 & -2.409412 & 0.052235 \\
\hline 6 & 6 & 0 & 0.052411 & -0.537512 & 1.519479 \\
\hline 7 & 6 & 0 & -0.651209 & 0.067520 & 0.344890 \\
\hline 8 & 6 & 0 & -0.114706 & 1.443394 & 0.302161 \\
\hline 9 & 6 & 0 & -0.364033 & 2.563080 & -0.484533 \\
\hline 10 & 1 & 0 & -1.200641 & 2.578430 & -1.194412 \\
\hline 11 & 6 & 0 & 0.459238 & 3.695276 & -0.376779 \\
\hline 12 & 6 & 0 & 1.535644 & 3.671538 & 0.520116 \\
\hline 13 & 1 & 0 & 2.183301 & 4.554574 & 0.595320 \\
\hline 14 & 6 & 0 & 1.796105 & 2.562735 & 1.331673 \\
\hline 15 & 1 & 0 & 2.624217 & 2.547197 & 2.047864 \\
\hline 16 & 6 & 0 & 0.952519 & 1.475512 & 1.204861 \\
\hline 17 & 6 & 0 & 0.256014 & -0.820694 & -1.090749 \\
\hline 18 & 6 & 0 & 1.594890 & -0.259388 & -1.188850 \\
\hline 19 & 1 & 0 & 1.724387 & 0.776266 & -1.530936 \\
\hline 20 & 6 & 0 & 2.680553 & -0.963791 & -0.752941 \\
\hline 21 & 6 & 0 & 2.552251 & -2.330521 & -0.383176 \\
\hline 22 & 6 & 0 & 1.308103 & -2.961560 & -0.508274 \\
\hline 23 & 6 & 0 & 0.177786 & -2.269156 & -0.897910 \\
\hline 24 & 6 & 0 & -2.113061 & -0.170375 & 0.183740 \\
\hline 25 & 6 & 0 & -2.874860 & -0.819347 & 1.167660 \\
\hline 26 & 1 & 0 & -2.385737 & -1.185160 & 2.075589 \\
\hline 27 & 6 & 0 & -4.240707 & -1.024681 & 0.992918 \\
\hline 28 & 1 & 0 & -4.811740 & -1.535868 & 1.776816 \\
\hline 29 & 6 & 0 & -4.881138 & -0.589557 & -0.165852 \\
\hline 30 & 1 & 0 & -5.955604 & -0.757357 & -0.302322 \\
\hline 31 & 6 & 0 & -4.140383 & 0.064585 & -1.147955 \\
\hline 32 & 1 & 0 & -4.628816 & 0.416173 & -2.064323 \\
\hline 33 & 6 & 0 & -2.774882 & 0.272811 & -0.972566 \\
\hline 34 & 1 & 0 & -2.213185 & 0.777768 & -1.768212 \\
\hline 35 & 6 & 0 & 0.167786 & 4.921387 & -1.191449 \\
\hline 36 & 1 & 0 & -0.235693 & 4.663381 & -2.187768 \\
\hline 37 & 1 & 0 & 1.072832 & 5.537417 & -1.340616 \\
\hline 38 & 1 & 0 & -0.585581 & 5.566278 & -0.697313 \\
\hline 39 & 1 & 0 & 1.259743 & -4.035769 & -0.293122 \\
\hline 40 & 6 & 0 & -1.131455 & -2.971663 & -1.049894 \\
\hline 41 & 1 & 0 & -1.728135 & -2.556636 & -1.884522 \\
\hline 42 & 1 & 0 & -0.984338 & -4.052356 & -1.226655 \\
\hline 43 & 1 & 0 & -1.752734 & -2.868568 & -0.137431 \\
\hline 44 & 8 & 0 & 1.064891 & 0.314524 & 1.911462 \\
\hline 45 & 1 & 0 & -0.444399 & -0.435980 & -1.851517 \\
\hline
\end{tabular}

Cartesian coordinates (in Å) and energy of TS-C(5)-C $\mathrm{E}(\mathrm{UM} 06)=-1148.97135646 \mathrm{au}$

\begin{tabular}{lllcc} 
Center & Atomic & Atomic & \multicolumn{2}{c}{ Coordinates (Angstroms) } \\
number & number & type & $X$ & $Y$
\end{tabular}




\begin{tabular}{|c|c|c|c|c|c|}
\hline 1 & 8 & 0 & 0.116003 & 2.962284 & -1.281769 \\
\hline 2 & 8 & 0 & 3.971723 & -0.056087 & -1.158853 \\
\hline 3 & 1 & 0 & 4.038698 & 0.909313 & -1.240423 \\
\hline 4 & 8 & 0 & 3.413977 & -2.455781 & -0.185156 \\
\hline 5 & 1 & 0 & 4.214919 & -1.896802 & -0.187310 \\
\hline 6 & 6 & 0 & -0.641417 & 2.141382 & -0.848385 \\
\hline 7 & 6 & 0 & -0.362596 & 0.852392 & -0.115941 \\
\hline 8 & 6 & 0 & -1.728863 & 0.369213 & 0.217888 \\
\hline 9 & 6 & 0 & -2.251346 & -0.690379 & 0.954568 \\
\hline & 1 & 0 & -1.602388 & -1.380233 & 1.505255 \\
\hline & 6 & 0 & -3.635343 & -0.921073 & 0.984129 \\
\hline & 6 & 0 & -4.485223 & -0.070472 & 0.269519 \\
\hline & 1 & 0 & -5.566606 & -0.255174 & 0.290567 \\
\hline & 6 & 0 & -3.990835 & 1.014123 & -0.460920 \\
\hline & 1 & 0 & -4.647950 & 1.697199 & -1.008035 \\
\hline 6 & 6 & 0 & -2.623601 & 1.203590 & -0.459905 \\
\hline 7 & 6 & 0 & 0.269280 & -0.104887 & -1.569025 \\
\hline & 6 & 0 & 1.655627 & 0.323282 & -1.745207 \\
\hline & 1 & 0 & 1.858097 & 1.331425 & -2.128863 \\
\hline & 6 & 0 & 2.667300 & -0.436742 & -1.234725 \\
\hline & 6 & 0 & 2.416112 & -1.746282 & -0.735065 \\
\hline & 6 & 0 & 1.131502 & -2.296044 & -0.857919 \\
\hline & 6 & 0 & 0.076387 & -1.546754 & -1.339027 \\
\hline & 6 & 0 & 0.721180 & 0.856737 & 0.915870 \\
\hline & 6 & 0 & 1.619562 & 1.929168 & 1.044623 \\
\hline & 1 & 0 & 1.487428 & 2.820416 & 0.424610 \\
\hline & 6 & 0 & 2.682962 & 1.870545 & 1.942191 \\
\hline & 1 & 0 & 3.361899 & 2.727751 & 2.027360 \\
\hline & 6 & 0 & 2.886681 & 0.738062 & 2.728589 \\
\hline & 1 & 0 & 3.728374 & 0.691615 & 3.429363 \\
\hline & 6 & 0 & 1.999801 & -0.329809 & 2.619291 \\
\hline 2 & 1 & 0 & 2.141011 & -1.230037 & 3.228639 \\
\hline 3 & 6 & 0 & 0.930013 & -0.266235 & 1.729800 \\
\hline 4 & 1 & 0 & 0.274838 & -1.136115 & 1.642924 \\
\hline 5 & 6 & 0 & -4.174315 & -2.088208 & 1.758358 \\
\hline 6 & 1 & 0 & -3.823728 & -3.050431 & 1.335821 \\
\hline 7 & 1 & 0 & -5.278391 & -2.107466 & 1.753188 \\
\hline 8 & 1 & 0 & -3.844117 & -2.064035 & 2.814214 \\
\hline 9 & 1 & 0 & 1.001899 & -3.356462 & -0.604584 \\
\hline 0 & 6 & 0 & -1.221099 & -2.184991 & -1.723859 \\
\hline 1 & 1 & 0 & -2.079255 & -1.494937 & -1.633416 \\
\hline 2 & 1 & 0 & -1.188450 & -2.513852 & -2.784463 \\
\hline 3 & 1 & 0 & -1.436373 & -3.082802 & -1.113868 \\
\hline 4 & 8 & 0 & -1.985949 & 2.226251 & -1.105763 \\
\hline 5 & 1 & 0 & -0.416551 & 0.277572 & -2.347738 \\
\hline
\end{tabular}

Cartesian coordinates (in A) and energy of TS-C(5)-D

$\mathrm{E}(\mathrm{UM} 06)=-1148.97115418 \mathrm{au}$

\begin{tabular}{rrrrrr}
$\begin{array}{c}\text { Center } \\
\text { number }\end{array}$ & $\begin{array}{c}\text { Atomic } \\
\text { number }\end{array}$ & $\begin{array}{c}\text { Atomic } \\
\text { type }\end{array}$ & \multicolumn{3}{c}{ Coordinates (Angstroms) } \\
\hline 1 & 8 & 0 & -1.492622 & -0.034016 & 1.967911 \\
2 & 8 & 0 & -4.371625 & -0.146049 & -0.226753 \\
3 & 1 & 0 & -4.385439 & 0.818595 & -0.120046 \\
4 & 8 & 0 & -3.913878 & -2.735519 & 0.079526 \\
5 & 1 & 0 & -4.666233 & -2.155644 & 0.305492 \\
6 & 6 & 0 & -0.415469 & -0.216271 & 1.475535 \\
7 & 6 & 0 & 0.237728 & 0.464860 & 0.313419 \\
8 & 6 & 0 & 1.594301 & -0.117189 & 0.299247 \\
9 & 6 & 0 & 2.752204 & 0.106535 & -0.442624 \\
10 & 1 & 0 & 2.799791 & 0.905332 & -1.192371 \\
11 & 6 & 0 & 3.885989 & -0.692372 & -0.235499 \\
12 & 6 & 0 & 3.834089 & -1.715835 & 0.720556 \\
13 & 1 & 0 & 4.720633 & -2.344316 & 0.874387 \\
14 & 6 & 0 & 2.690856 & -1.949298 & 1.489684 \\
15 & 1 & 0 & 2.649157 & -2.738150 & 2.247237 \\
16 & 6 & 0 & 1.597987 & -1.137477 & 1.256857 \\
17 & 6 & 0 & -0.765124 & -0.289569 & -1.144949 \\
18 & 6 & 0 & -2.108036 & 0.233874 & -0.990930
\end{tabular}




\begin{tabular}{|c|c|c|c|c|c|}
\hline 19 & 1 & 0 & -2.284869 & 1.309085 & -1.129630 \\
\hline 20 & 6 & 0 & -3.115843 & -0.565038 & -0.532668 \\
\hline 21 & 6 & 0 & -2.915986 & -1.964657 & -0.374941 \\
\hline 22 & 6 & 0 & -1.693958 & -2.528972 & -0.753841 \\
\hline 23 & 6 & 0 & -0.642049 & -1.746900 & -1.199951 \\
\hline 24 & 6 & 0 & 0.051378 & 1.935469 & 0.152894 \\
\hline 25 & 6 & 0 & -0.674269 & 2.701162 & 1.078025 \\
\hline 26 & 1 & 0 & -1.120367 & 2.214012 & 1.950003 \\
\hline 27 & 6 & 0 & -0.840826 & 4.072299 & 0.895951 \\
\hline 28 & 1 & 0 & -1.405375 & 4.647841 & 1.639218 \\
\hline 29 & 6 & 0 & -0.297487 & 4.712931 & -0.215410 \\
\hline 30 & 1 & 0 & -0.432010 & 5.791579 & -0.356056 \\
\hline 31 & 6 & 0 & 0.417767 & 3.964313 & -1.148112 \\
\hline 32 & 1 & 0 & 0.847885 & 4.449825 & -2.031977 \\
\hline 33 & 6 & 0 & 0.587874 & 2.594809 & -0.965164 \\
\hline 34 & 1 & 0 & 1.138133 & 2.027882 & -1.725449 \\
\hline 35 & 6 & 0 & 5.147278 & -0.431222 & -1.005787 \\
\hline 36 & 1 & 0 & 4.934319 & -0.048642 & -2.020646 \\
\hline 37 & 1 & 0 & 5.758159 & -1.346101 & -1.109600 \\
\hline 38 & 1 & 0 & 5.780766 & 0.325766 & -0.502345 \\
\hline 39 & 1 & 0 & -1.608860 & -3.622385 & -0.727754 \\
\hline 40 & 6 & 0 & 0.599576 & -2.380345 & -1.745350 \\
\hline 41 & 1 & 0 & 1.023803 & -1.782882 & -2.575016 \\
\hline 42 & 1 & 0 & 1.403518 & -2.477582 & -0.989037 \\
\hline 43 & 1 & 0 & 0.387576 & -3.395896 & -2.126748 \\
\hline 44 & 8 & 0 & 0.408615 & -1.223692 & 1.920714 \\
\hline 45 & 1 & 0 & -0.152386 & 0.229256 & -1.902503 \\
\hline
\end{tabular}

Cartesian coordinates (in Å) and energy of TS-C(5)-E

$\mathrm{E}(\mathrm{UM} 06)=-1148.96918963 \mathrm{au}$

\begin{tabular}{|c|c|c|c|c|c|}
\hline \multirow{2}{*}{$\begin{array}{l}\text { Center } \\
\text { number }\end{array}$} & \multirow{2}{*}{$\begin{array}{l}\text { Atomic } \\
\text { number }\end{array}$} & \multirow{2}{*}{$\begin{array}{l}\text { Atomic } \\
\text { type }\end{array}$} & \multicolumn{3}{|c|}{ Coordinates (Angstroms) } \\
\hline & & & $\mathrm{X}$ & $\mathrm{Y}$ & $\mathrm{Z}$ \\
\hline 1 & 8 & 0 & 0.027812 & 2.452061 & 1.769588 \\
\hline 2 & 8 & 0 & 2.582846 & -1.897283 & -1.935744 \\
\hline 3 & 1 & 0 & 1.917247 & -2.599817 & -1.861773 \\
\hline 4 & 8 & 0 & 4.261932 & 0.000899 & -1.160627 \\
\hline 5 & 1 & 0 & 4.359473 & -0.954668 & -1.337046 \\
\hline 6 & 6 & 0 & -0.734494 & 1.710293 & 1.223985 \\
\hline 7 & 6 & 0 & -0.484127 & 0.397017 & 0.527076 \\
\hline 8 & 6 & 0 & -1.860760 & -0.077231 & 0.222082 \\
\hline 9 & 6 & 0 & -2.400729 & -1.194084 & -0.416094 \\
\hline 10 & 1 & 0 & -1.764561 & -2.026713 & -0.740586 \\
\hline 11 & 6 & 0 & -3.778925 & -1.275081 & -0.668617 \\
\hline 12 & 6 & 0 & -4.602862 & -0.206065 & -0.298207 \\
\hline 13 & 1 & 0 & -5.679502 & -0.267163 & -0.501673 \\
\hline 14 & 6 & 0 & -4.086532 & 0.938370 & 0.312796 \\
\hline 15 & 1 & 0 & -4.719635 & 1.786089 & 0.592708 \\
\hline 16 & 6 & 0 & -2.726312 & 0.970728 & 0.550838 \\
\hline 17 & 6 & 0 & 0.184296 & 0.848022 & -1.152853 \\
\hline 18 & 6 & 0 & 0.687192 & -0.409012 & -1.698837 \\
\hline 19 & 1 & 0 & -0.021658 & -1.181113 & -2.027369 \\
\hline 20 & 6 & 0 & 2.021524 & -0.692751 & -1.638443 \\
\hline 21 & 6 & 0 & 2.960406 & 0.308973 & -1.264110 \\
\hline 22 & 6 & 0 & 2.516123 & 1.619584 & -1.038084 \\
\hline 23 & 6 & 0 & 1.171810 & 1.931218 & -1.053176 \\
\hline 24 & 6 & 0 & 0.532856 & -0.485323 & 1.175986 \\
\hline 25 & 6 & 0 & 1.719516 & 0.050020 & 1.703393 \\
\hline 26 & 1 & 0 & 1.850770 & 1.134279 & 1.734932 \\
\hline 27 & 6 & 0 & 2.729238 & -0.782455 & 2.180411 \\
\hline 28 & 1 & 0 & 3.649790 & -0.336220 & 2.574524 \\
\hline 29 & 6 & 0 & 2.578227 & -2.167201 & 2.156927 \\
\hline 30 & 1 & 0 & 3.375844 & -2.819619 & 2.531092 \\
\hline 31 & 6 & 0 & 1.388250 & -2.711657 & 1.677418 \\
\hline 32 & 1 & 0 & 1.235176 & -3.797717 & 1.687266 \\
\hline 33 & 6 & 0 & 0.379687 & -1.879995 & 1.196250 \\
\hline 34 & 1 & 0 & -0.554422 & -2.332750 & 0.850894 \\
\hline 35 & 6 & 0 & -4.358884 & -2.504003 & -1.305410 \\
\hline 36 & 1 & 0 & -3.655725 & -2.961774 & -2.025270 \\
\hline
\end{tabular}




$\begin{array}{lllrrr}37 & 1 & 0 & -5.296307 & -2.279266 & -1.845227 \\ 38 & 1 & 0 & -4.597828 & -3.279281 & -0.550630 \\ 39 & 1 & 0 & 3.273448 & 2.397869 & -0.879160 \\ 40 & 6 & 0 & 0.703529 & 3.351670 & -1.025466 \\ 41 & 1 & 0 & 1.325594 & 3.977342 & -0.360290 \\ 42 & 1 & 0 & 0.751039 & 3.795133 & -2.041892 \\ 43 & 1 & 0 & -0.344036 & 3.445717 & -0.687863 \\ 44 & 8 & 0 & -2.069199 & 2.028203 & 1.112434 \\ 45 & 1 & 0 & -0.766002 & 1.182400 & -1.605023\end{array}$

Cartesian coordinates (in $\AA$ ) and energy of TS-C(5)-F $\mathrm{E}(\mathrm{UM} 06)=-1148.97727024 \mathrm{au}$

\begin{tabular}{|c|c|c|c|c|c|}
\hline \multirow{2}{*}{$\begin{array}{l}\text { Center } \\
\text { number }\end{array}$} & \multirow{2}{*}{$\begin{array}{l}\text { Atomic } \\
\text { number }\end{array}$} & \multirow{2}{*}{$\begin{array}{l}\text { Atomic } \\
\text { type }\end{array}$} & \multicolumn{3}{|c|}{ Coordinates (Angstroms) } \\
\hline & & & $X$ & Y & $\mathrm{Z}$ \\
\hline 1 & 8 & 0 & 1.509280 & -2.565423 & -1.591639 \\
\hline 2 & 8 & 0 & -3.338936 & -1.919715 & 0.573677 \\
\hline 3 & 1 & 0 & -3.209304 & -2.591861 & -0.114972 \\
\hline 4 & 8 & 0 & -3.417564 & 0.306965 & 2.021478 \\
\hline 5 & 1 & 0 & -4.096349 & -0.199096 & 1.535216 \\
\hline 6 & 6 & 0 & 0.803816 & -1.603743 & -1.500589 \\
\hline 7 & 6 & 0 & 0.895870 & -0.428536 & -0.569982 \\
\hline 8 & 6 & 0 & -0.239534 & 0.416284 & -0.971310 \\
\hline 9 & 6 & 0 & -0.770610 & 1.612794 & -0.496056 \\
\hline 10 & 1 & 0 & -0.244738 & 2.191045 & 0.274913 \\
\hline 11 & 6 & 0 & -2.014762 & 2.065081 & -0.959838 \\
\hline 12 & 6 & 0 & -2.698358 & 1.307689 & -1.918935 \\
\hline 13 & 1 & 0 & -3.667615 & 1.667645 & -2.287257 \\
\hline 14 & 6 & 0 & -2.183750 & 0.105900 & -2.417301 \\
\hline 15 & 1 & 0 & -2.717405 & -0.488698 & -3.165839 \\
\hline 16 & 6 & 0 & -0.967137 & -0.312899 & -1.916110 \\
\hline 17 & 6 & 0 & 0.302511 & -1.292311 & 1.051033 \\
\hline 18 & 6 & 0 & -0.921139 & -1.999368 & 0.718811 \\
\hline 19 & 1 & 0 & -0.866011 & -2.929530 & 0.136867 \\
\hline 20 & 6 & 0 & -2.137895 & -1.435468 & 0.988822 \\
\hline 21 & 6 & 0 & -2.225010 & -0.268692 & 1.792856 \\
\hline 22 & 6 & 0 & -1.061117 & 0.252711 & 2.374278 \\
\hline 23 & 6 & 0 & 0.188009 & -0.260088 & 2.079148 \\
\hline 24 & 6 & 0 & 2.261510 & 0.107631 & -0.334863 \\
\hline 25 & 6 & 0 & 3.318869 & -0.750514 & 0.011674 \\
\hline 26 & 1 & 0 & 3.146783 & -1.831007 & 0.054419 \\
\hline 27 & 6 & 0 & 4.586684 & -0.244716 & 0.280166 \\
\hline 28 & 1 & 0 & 5.395140 & -0.933082 & 0.553087 \\
\hline 29 & 6 & 0 & 4.834199 & 1.125454 & 0.199691 \\
\hline 30 & 1 & 0 & 5.832989 & 1.521442 & 0.416535 \\
\hline 31 & 6 & 0 & 3.803839 & 1.983263 & -0.180026 \\
\hline 32 & 1 & 0 & 3.990077 & 3.059469 & -0.275543 \\
\hline 33 & 6 & 0 & 2.533648 & 1.479387 & -0.448808 \\
\hline 34 & 1 & 0 & 1.741482 & 2.164760 & -0.768729 \\
\hline 35 & 6 & 0 & -2.624211 & 3.302749 & -0.371015 \\
\hline 36 & 1 & 0 & -3.056481 & 3.086512 & 0.626858 \\
\hline 37 & 1 & 0 & -3.437022 & 3.702411 & -1.003494 \\
\hline 38 & 1 & 0 & -1.875080 & 4.104283 & -0.233728 \\
\hline 39 & 1 & 0 & -1.175763 & 1.076151 & 3.090995 \\
\hline 40 & 6 & 0 & 1.411214 & 0.255062 & 2.768101 \\
\hline 41 & 1 & 0 & 2.000175 & 0.944020 & 2.128956 \\
\hline 42 & 1 & 0 & 1.145289 & 0.801214 & 3.691087 \\
\hline 43 & 1 & 0 & 2.098060 & -0.569609 & 3.039488 \\
\hline 44 & 8 & 0 & -0.349801 & -1.489925 & -2.243609 \\
\hline 45 & 1 & 0 & 1.192058 & -1.937308 & 1.143393 \\
\hline
\end{tabular}

Cartesian coordinates (in Å) and energy of INT-II-C(5)-A $\mathrm{E}(\mathrm{UM} 06)=-1148.98075385 \mathrm{au}$

\begin{tabular}{lllcc} 
Center & Atomic & Atomic & \multicolumn{2}{c}{ Coordinates (Angstroms) } \\
number & number & type & $X$ & $Y$
\end{tabular}




\begin{tabular}{|c|c|c|c|c|c|}
\hline 1 & 8 & 0 & -2.022155 & -1.949532 & -1.959375 \\
\hline 2 & 8 & 0 & 1.330221 & 3.535646 & 0.254667 \\
\hline 3 & 1 & 0 & 0.487429 & 3.924712 & 0.538256 \\
\hline 4 & 8 & 0 & 3.502355 & 2.254675 & -0.574538 \\
\hline 5 & 1 & 0 & 3.360660 & 3.066106 & -0.052329 \\
\hline 6 & 6 & 0 & -1.217817 & -1.782167 & -1.095247 \\
\hline 7 & 6 & 0 & -0.856669 & -0.477345 & -0.371956 \\
\hline 8 & 6 & 0 & 0.234994 & -0.940236 & 0.547846 \\
\hline 9 & 6 & 0 & 1.078659 & -0.281223 & 1.431675 \\
\hline & 1 & 0 & 0.948809 & 0.789464 & 1.637350 \\
\hline & 6 & 0 & 2.132350 & -0.973974 & 2.047523 \\
\hline & 6 & 0 & 2.293894 & -2.337905 & 1.781202 \\
\hline & 1 & 0 & 3.116141 & -2.880249 & 2.264871 \\
\hline & 6 & 0 & 1.445722 & -3.025579 & 0.908421 \\
\hline & 1 & 0 & 1.574541 & -4.090088 & 0.689878 \\
\hline & 6 & 0 & 0.438864 & -2.296003 & 0.306868 \\
\hline 7 & 6 & 0 & -0.284988 & 0.579063 & -1.445097 \\
\hline & 6 & 0 & -0.124127 & 1.905063 & -0.775544 \\
\hline & 1 & 0 & -1.026272 & 2.433794 & -0.438217 \\
\hline & 6 & 0 & 1.103623 & 2.407156 & -0.476688 \\
\hline & 6 & 0 & 2.294761 & 1.767735 & -0.926214 \\
\hline & 6 & 0 & 2.190295 & 0.664041 & -1.790530 \\
\hline & 6 & 0 & 0.980740 & 0.097463 & -2.112135 \\
\hline & 6 & 0 & -2.110972 & 0.080536 & 0.285561 \\
\hline & 6 & 0 & -3.228913 & 0.394532 & -0.500664 \\
\hline & 1 & 0 & -3.215855 & 0.179186 & -1.574450 \\
\hline & 6 & 0 & -4.368765 & 0.950064 & 0.072902 \\
\hline & 1 & 0 & -5.232180 & 1.184538 & -0.560603 \\
\hline & 6 & 0 & -4.418178 & 1.200582 & 1.443919 \\
\hline & 1 & 0 & -5.316686 & 1.637862 & 1.894540 \\
\hline & 6 & 0 & -3.319539 & 0.878865 & 2.235401 \\
\hline 2 & 1 & 0 & -3.348356 & 1.056725 & 3.316885 \\
\hline 3 & 6 & 0 & -2.176757 & 0.321870 & 1.660946 \\
\hline 4 & 1 & 0 & -1.326772 & 0.062524 & 2.301479 \\
\hline 5 & 6 & 0 & 3.080167 & -0.228323 & 2.939720 \\
\hline 6 & 1 & 0 & 3.585803 & 0.583172 & 2.380241 \\
\hline 7 & 1 & 0 & 3.862841 & -0.889218 & 3.351970 \\
\hline 8 & 1 & 0 & 2.556319 & 0.244886 & 3.792320 \\
\hline 9 & 1 & 0 & 3.118902 & 0.280581 & -2.232424 \\
\hline 0 & 6 & 0 & 0.894582 & -1.004724 & -3.119167 \\
\hline 1 & 1 & 0 & 0.750357 & -2.005221 & -2.663389 \\
\hline 2 & 1 & 0 & 0.038831 & -0.857688 & -3.806493 \\
\hline 3 & 1 & 0 & 1.819121 & -1.060904 & -3.721105 \\
\hline 4 & 8 & 0 & -0.439720 & -2.801913 & -0.620866 \\
\hline 5 & 1 & 0 & -1.090807 & 0.631870 & -2.210329 \\
\hline
\end{tabular}

Cartesian coordinates (in A) and energy of INT-II-C(5)-B $\mathrm{E}(\mathrm{UM} 06)=-1148.97525867 \mathrm{au}$

\begin{tabular}{cccccc}
$\begin{array}{c}\text { Center } \\
\text { number }\end{array}$ & $\begin{array}{c}\text { Atomic } \\
\text { number }\end{array}$ & $\begin{array}{c}\text { Atomic } \\
\text { type }\end{array}$ & \multicolumn{3}{c}{ Cordinates (Angstroms) } \\
\hline 1 & 8 & 0 & 0.930048 & -1.121441 & Y \\
2 & 8 & 0 & 3.768005 & 1.860667 & -0.564745 \\
3 & 1 & 0 & 3.244900 & 2.667855 & -0.693252 \\
4 & 8 & 0 & 4.937662 & -0.458651 & -0.006966 \\
5 & 1 & 0 & 5.246233 & 0.466118 & 0.011830 \\
6 & 6 & 0 & 0.348047 & -0.243124 & 1.543022 \\
7 & 6 & 0 & -0.370434 & -0.281246 & 0.192019 \\
8 & 6 & 0 & -0.958764 & 1.103951 & 0.135763 \\
9 & 6 & 0 & -1.757007 & 1.758176 & -0.791006 \\
10 & 1 & 0 & -2.132420 & 1.234063 & -1.679821 \\
11 & 6 & 0 & -2.093450 & 3.107628 & -0.597641 \\
12 & 6 & 0 & -1.603744 & 3.770931 & 0.534823 \\
13 & 1 & 0 & -1.862071 & 4.827284 & 0.683464 \\
14 & 6 & 0 & -0.802749 & 3.127306 & 1.481377 \\
15 & 1 & 0 & -0.422433 & 3.642694 & 2.368894 \\
16 & 6 & 0 & -0.502339 & 1.797387 & 1.252372 \\
17 & 6 & 0 & 0.789164 & -0.444102 & -0.922530 \\
18 & 6 & 0 & 1.643457 & 0.785648 & -0.958050 \\
19 & 1 & 0 & 1.175221 & 1.741219 & -1.231812
\end{tabular}




\begin{tabular}{|c|c|c|c|c|c|}
\hline 20 & 6 & 0 & 2.960835 & 0.765696 & -0.618700 \\
\hline 21 & 6 & 0 & 3.631419 & -0.457951 & -0.333323 \\
\hline 22 & 6 & 0 & 2.927076 & -1.664268 & -0.469064 \\
\hline 23 & 6 & 0 & 1.588628 & -1.715017 & -0.777378 \\
\hline 24 & 6 & 0 & -1.442688 & -1.352723 & 0.107353 \\
\hline 25 & 6 & 0 & -1.832583 & -2.112606 & 1.216056 \\
\hline 26 & 1 & 0 & -1.330328 & -1.979769 & 2.179730 \\
\hline 27 & 6 & 0 & -2.839145 & -3.070537 & 1.107109 \\
\hline 28 & 1 & 0 & -3.119843 & -3.657752 & 1.989121 \\
\hline 29 & 6 & 0 & -3.480283 & -3.287134 & -0.109697 \\
\hline 30 & 1 & 0 & -4.267290 & -4.045125 & -0.194767 \\
\hline 31 & 6 & 0 & -3.113253 & -2.525522 & -1.217380 \\
\hline 32 & 1 & 0 & -3.611560 & -2.678937 & -2.181710 \\
\hline 33 & 6 & 0 & -2.109743 & -1.567230 & -1.106251 \\
\hline 34 & 1 & 0 & -1.832954 & -0.990057 & -1.997318 \\
\hline 35 & 6 & 0 & -2.989368 & 3.812394 & -1.573234 \\
\hline 36 & 1 & 0 & -2.817113 & 3.466802 & -2.608994 \\
\hline 37 & 1 & 0 & -2.837302 & 4.906450 & -1.550436 \\
\hline 38 & 1 & 0 & -4.058248 & 3.628452 & -1.346868 \\
\hline 39 & 1 & 0 & 3.495050 & -2.593146 & -0.333451 \\
\hline 40 & 6 & 0 & 0.915439 & -3.034437 & -0.970128 \\
\hline 41 & 1 & 0 & 0.258128 & -3.035739 & -1.862490 \\
\hline 42 & 1 & 0 & 1.662333 & -3.839868 & -1.089669 \\
\hline 43 & 1 & 0 & 0.269655 & -3.307820 & -0.111267 \\
\hline 44 & 8 & 0 & 0.274946 & 1.020880 & 2.069247 \\
\hline 45 & 1 & 0 & 0.203657 & -0.503682 & -1.869070 \\
\hline
\end{tabular}

Cartesian coordinates (in Å) and energy of INT-II-C(5)-C

$\mathrm{E}(\mathrm{UM} 06)=-1148.97203307 \mathrm{au}$

\begin{tabular}{|c|c|c|c|c|c|}
\hline \multirow{2}{*}{$\begin{array}{l}\text { Center } \\
\text { number }\end{array}$} & \multirow{2}{*}{$\begin{array}{l}\text { Atomic } \\
\text { number }\end{array}$} & \multirow{2}{*}{$\begin{array}{l}\text { Atomic } \\
\text { type }\end{array}$} & \multicolumn{3}{|c|}{ Coordinates (Angstroms) } \\
\hline & & & $\mathrm{X}$ & Y & $\mathrm{Z}$ \\
\hline 1 & 8 & 0 & -0.101096 & -2.832561 & -1.561524 \\
\hline 2 & 8 & 0 & -4.012572 & 0.122570 & -1.096443 \\
\hline 3 & 1 & 0 & -4.082694 & -0.829061 & -1.275654 \\
\hline 4 & 8 & 0 & -3.449991 & 2.446856 & 0.046112 \\
\hline 5 & 1 & 0 & -4.248285 & 1.885852 & 0.011868 \\
\hline 6 & 6 & 0 & 0.649132 & -2.055751 & -1.048727 \\
\hline 7 & 6 & 0 & 0.337497 & -0.765427 & -0.290263 \\
\hline 8 & 6 & 0 & 1.716693 & -0.319888 & 0.113361 \\
\hline 9 & 6 & 0 & 2.222864 & 0.729650 & 0.872484 \\
\hline 10 & 1 & 0 & 1.560259 & 1.454631 & 1.358598 \\
\hline 11 & 6 & 0 & 3.608472 & 0.914177 & 0.997097 \\
\hline 12 & 6 & 0 & 4.474898 & 0.026100 & 0.351120 \\
\hline 13 & 1 & 0 & 5.558102 & 0.172851 & 0.446332 \\
\hline 14 & 6 & 0 & 3.994461 & -1.044525 & -0.406427 \\
\hline 15 & 1 & 0 & 4.662264 & -1.752370 & -0.906941 \\
\hline 16 & 6 & 0 & 2.623580 & -1.184981 & -0.498120 \\
\hline 17 & 6 & 0 & -0.290458 & 0.191018 & -1.514567 \\
\hline 18 & 6 & 0 & -1.704975 & -0.214162 & -1.728625 \\
\hline 19 & 1 & 0 & -1.911967 & -1.192059 & -2.181725 \\
\hline 20 & 6 & 0 & -2.707403 & 0.507518 & -1.158685 \\
\hline 21 & 6 & 0 & -2.455858 & 1.782934 & -0.569468 \\
\hline 22 & 6 & 0 & -1.181463 & 2.360912 & -0.701174 \\
\hline 23 & 6 & 0 & -0.127291 & 1.655024 & -1.236080 \\
\hline 24 & 6 & 0 & -0.685135 & -0.913521 & 0.819793 \\
\hline 25 & 6 & 0 & -1.520258 & -2.035622 & 0.921428 \\
\hline 26 & 1 & 0 & -1.395002 & -2.872285 & 0.228179 \\
\hline 27 & 6 & 0 & -2.528113 & -2.095145 & 1.882181 \\
\hline 28 & 1 & 0 & -3.161664 & -2.988405 & 1.940809 \\
\hline 29 & 6 & 0 & -2.734025 & -1.033720 & 2.760312 \\
\hline 30 & 1 & 0 & -3.532536 & -1.079164 & 3.509948 \\
\hline 31 & 6 & 0 & -1.905721 & 0.082279 & 2.678229 \\
\hline 32 & 1 & 0 & -2.049172 & 0.928971 & 3.359436 \\
\hline 33 & 6 & 0 & -0.891349 & 0.134968 & 1.725174 \\
\hline 34 & 1 & 0 & -0.281491 & 1.039316 & 1.667023 \\
\hline 35 & 6 & 0 & 4.132683 & 2.068808 & 1.799462 \\
\hline 36 & 1 & 0 & 3.829651 & 3.038696 & 1.358694 \\
\hline 37 & 1 & 0 & 5.235449 & 2.060256 & 1.855320 \\
\hline
\end{tabular}




$\begin{array}{rrrrrr}38 & 1 & 0 & 3.744547 & 2.053728 & 2.835610 \\ 39 & 1 & 0 & -1.068004 & 3.416932 & -0.421013 \\ 40 & 6 & 0 & 1.126144 & 2.336756 & -1.684207 \\ 41 & 1 & 0 & 2.024420 & 1.699608 & -1.584778 \\ 42 & 1 & 0 & 1.052544 & 2.607746 & -2.759736 \\ 43 & 1 & 0 & 1.305296 & 3.275436 & -1.126277 \\ 44 & 8 & 0 & 2.000458 & -2.188364 & -1.191917 \\ 45 & 1 & 0 & 0.337654 & -0.073102 & -2.391310\end{array}$

Cartesian coordinates (in $\AA$ ) and energy of INT-II-C(5)-D $\mathrm{E}(\mathrm{UM} 06)=-1148.94668047 \mathrm{au}$

\begin{tabular}{|c|c|c|c|c|c|}
\hline \multirow{2}{*}{$\begin{array}{l}\text { Center } \\
\text { number }\end{array}$} & \multirow{2}{*}{$\begin{array}{l}\text { Atomic } \\
\text { number }\end{array}$} & \multirow{2}{*}{$\begin{array}{l}\text { Atomic } \\
\text { type }\end{array}$} & \multicolumn{3}{|c|}{ Coordinates (Angstroms) } \\
\hline & & & $\mathrm{X}$ & $\mathrm{Y}$ & $\mathrm{Z}$ \\
\hline 1 & 8 & 0 & -1.427406 & 0.060952 & 2.029272 \\
\hline 2 & 8 & 0 & -4.447390 & 0.420281 & -0.412359 \\
\hline 3 & 1 & 0 & -4.333070 & 1.383165 & -0.449414 \\
\hline 4 & 8 & 0 & -4.417809 & -2.180757 & 0.110950 \\
\hline 5 & 1 & 0 & -5.091324 & -1.482300 & 0.211838 \\
\hline 6 & 6 & 0 & -0.387819 & -0.196105 & 1.507255 \\
\hline 7 & 6 & 0 & 0.169557 & 0.307655 & 0.171166 \\
\hline 8 & 6 & 0 & 1.539524 & -0.323703 & 0.172261 \\
\hline 9 & 6 & 0 & 2.609035 & -0.265931 & -0.709839 \\
\hline 10 & 1 & 0 & 2.572015 & 0.365617 & -1.603743 \\
\hline 11 & 6 & 0 & 3.767962 & -1.015722 & -0.455729 \\
\hline 12 & 6 & 0 & 3.809146 & -1.822908 & 0.684819 \\
\hline 13 & 1 & 0 & 4.702997 & -2.423964 & 0.880447 \\
\hline 14 & 6 & 0 & 2.747967 & -1.884151 & 1.591940 \\
\hline 15 & 1 & 0 & 2.776699 & -2.514545 & 2.492596 \\
\hline 16 & 6 & 0 & 1.628536 & -1.118080 & 1.300479 \\
\hline 17 & 6 & 0 & -0.785803 & -0.297731 & -0.985367 \\
\hline 18 & 6 & 0 & -2.095017 & 0.421168 & -0.969340 \\
\hline 19 & 1 & 0 & -2.112847 & 1.496689 & -1.192083 \\
\hline 20 & 6 & 0 & -3.243356 & -0.188023 & -0.564810 \\
\hline 21 & 6 & 0 & -3.272359 & -1.587600 & -0.296782 \\
\hline 22 & 6 & 0 & -2.124367 & -2.357681 & -0.549480 \\
\hline 23 & 6 & 0 & -0.935940 & -1.800687 & -0.944825 \\
\hline 24 & 6 & 0 & 0.228763 & 1.814741 & 0.080380 \\
\hline 25 & 6 & 0 & -0.094866 & 2.659551 & 1.144679 \\
\hline 26 & 1 & 0 & -0.454587 & 2.231389 & 2.089153 \\
\hline 27 & 6 & 0 & -0.004714 & 4.046050 & 1.027316 \\
\hline 28 & 1 & 0 & -0.262124 & 4.677243 & 1.885897 \\
\hline 29 & 6 & 0 & 0.424000 & 4.617081 & -0.165686 \\
\hline 30 & 1 & 0 & 0.490437 & 5.706702 & -0.255816 \\
\hline 31 & 6 & 0 & 0.743218 & 3.796515 & -1.246564 \\
\hline 32 & 1 & 0 & 1.071278 & 4.236563 & -2.195543 \\
\hline 33 & 6 & 0 & 0.646831 & 2.413405 & -1.116842 \\
\hline 34 & 1 & 0 & 0.893757 & 1.791210 & -1.985889 \\
\hline 35 & 6 & 0 & 4.960958 & -0.937304 & -1.357860 \\
\hline 36 & 1 & 0 & 4.678372 & -0.277350 & -2.370021 \\
\hline 37 & 1 & 0 & 5.292831 & -2.094215 & -1.663772 \\
\hline 38 & 1 & 0 & 5.910081 & -0.390949 & -0.751746 \\
\hline 39 & 1 & 0 & -2.227031 & -3.447776 & -0.471981 \\
\hline 40 & 6 & 0 & 0.196618 & -2.664642 & -1.396632 \\
\hline 41 & 1 & 0 & 0.668224 & -2.268086 & -2.318767 \\
\hline 42 & 1 & 0 & 1.023191 & -2.754109 & -0.652786 \\
\hline 43 & 1 & 0 & -0.151394 & -3.687939 & -1.611486 \\
\hline 44 & 8 & 0 & 0.493923 & -1.076744 & 2.066353 \\
\hline 45 & 1 & 0 & -0.225301 & -0.032578 & -1.913849 \\
\hline
\end{tabular}

Cartesian coordinates (in $\AA$ ) and energy of INT-II-C(5)-E

$\mathrm{E}(\mathrm{UM} 06)=-1148.97013129 \mathrm{au}$

\begin{tabular}{cccccc}
$\begin{array}{l}\text { Center } \\
\text { number }\end{array}$ & $\begin{array}{c}\text { Atomic } \\
\text { number }\end{array}$ & $\begin{array}{c}\text { Atomic } \\
\text { type }\end{array}$ & $\mathrm{X}$ & \multicolumn{2}{c}{ Coordinates (Angstroms) } \\
-1 & 8 & 0 & 0.064215 & 2.428562 & 1.769101
\end{tabular}




$\begin{array}{rrrrrr}2 & 8 & 0 & 2.639055 & -1.883120 & -1.971507 \\ 3 & 1 & 0 & 1.963628 & -2.579734 & -1.963159 \\ 4 & 8 & 0 & 4.320748 & -0.026266 & -1.099835 \\ 5 & 1 & 0 & 4.414575 & -0.974245 & -1.312682 \\ 6 & 6 & 0 & -0.708408 & 1.712713 & 1.210053 \\ 7 & 6 & 0 & -0.446119 & 0.440073 & 0.401251 \\ 8 & 6 & 0 & -1.849202 & -0.027063 & 0.124486 \\ 9 & 6 & 0 & -2.396337 & -1.099523 & -0.575863 \\ 10 & 1 & 0 & -1.758216 & -1.887211 & -0.995463 \\ 11 & 6 & 0 & -3.783956 & -1.194335 & -0.765035 \\ 12 & 6 & 0 & -4.608030 & -0.181161 & -0.261972 \\ 13 & 1 & 0 & -5.692440 & -0.250891 & -0.415214 \\ 14 & 6 & 0 & -4.084427 & 0.918422 & 0.419360 \\ 15 & 1 & 0 & -4.718851 & 1.723137 & 0.803537 \\ 16 & 6 & 0 & -2.713445 & 0.963540 & 0.588487 \\ 17 & 6 & 0 & 0.220984 & 0.845416 & -1.079337 \\ 18 & 6 & 0 & 0.746300 & -0.403379 & -1.693490 \\ 19 & 1 & 0 & 0.044875 & -1.158004 & -2.074011 \\ 20 & 6 & 0 & 2.076275 & -0.689248 & -1.630894 \\ 21 & 6 & 0 & 3.020159 & 0.294360 & -1.213784 \\ 22 & 6 & 0 & 2.583641 & 1.611576 & -0.986731 \\ 23 & 6 & 0 & 1.247331 & 1.933689 & -0.995088 \\ 24 & 6 & 0 & 0.491538 & -0.497218 & 1.138162 \\ 25 & 6 & 0 & 1.683575 & -0.015887 & 1.697880 \\ 26 & 1 & 0 & 1.880525 & 1.059004 & 1.703814 \\ 27 & 6 & 0 & 2.623166 & -0.891296 & 2.237729 \\ 28 & 1 & 0 & 3.553086 & -0.488614 & 2.655844 \\ 29 & 6 & 0 & 2.388594 & -2.264318 & 2.247088 \\ 30 & 1 & 0 & 3.130774 & -2.951586 & 2.669807 \\ 31 & 6 & 0 & 1.188062 & -2.751545 & 1.735481 \\ 32 & 1 & 0 & 0.970685 & -3.826063 & 1.765643 \\ 33 & 6 & 0 & 0.250326 & -1.875682 & 1.191331 \\ 34 & 1 & 0 & -0.691774 & -2.283160 & 0.811746 \\ 35 & 6 & 0 & -4.371237 & -2.378589 & -1.475155 \\ 36 & 1 & 0 & -3.699155 & -2.753320 & -2.268761 \\ 37 & 1 & 0 & -5.341760 & -2.132798 & -1.942286 \\ 38 & 1 & 0 & -4.551138 & -3.222067 & -0.779481 \\ 39 & 1 & 0 & 3.347889 & 2.387094 & -0.845741 \\ 40 & 6 & 0 & 0.783196 & 3.354068 & -1.025677 \\ 41 & 1 & 0 & 1.482310 & 4.025889 & -0.495645 \\ 42 & 1 & 0 & 0.705401 & 3.713113 & -2.074071 \\ 43 & 1 & 0 & -0.217175 & 3.491169 & -0.575926 \\ 44 & 8 & 0 & -2.051201 & 1.988089 & 1.207655 \\ 45 & 1 & 0 & -0.657697 & 1.222498 & -1.640947 \\ --------------------------------------------------------------------\end{array}$

Cartesian coordinates (in $\AA$ ) and energy of INT-II-C(5)-F $\mathrm{E}(\mathrm{UM} 06)=-1148.98139631 \mathrm{au}$

\begin{tabular}{cccccc}
$\begin{array}{c}\text { Center } \\
\text { number }\end{array}$ & $\begin{array}{c}\text { Atomic } \\
\text { number }\end{array}$ & $\begin{array}{c}\text { Atomic } \\
\text { type }\end{array}$ & \multicolumn{3}{c}{ Coordinates (Angstroms) } \\
\hline 1 & 8 & 0 & -1.529225 & -0.152199 & Z \\
\hline 2 & 8 & 0 & 3.398906 & -1.595267 & 1.367332 \\
3 & 1 & 0 & 3.262630 & -1.411105 & 2.310496 \\
4 & 8 & 0 & 3.533001 & -1.709297 & -1.285575 \\
5 & 1 & 0 & 4.204286 & -1.604301 & -0.585380 \\
6 & 6 & 0 & -0.851101 & 0.350513 & 2.131641 \\
7 & 6 & 0 & -0.824272 & 0.046413 & 0.625704 \\
8 & 6 & 0 & 0.234867 & 0.997235 & 0.153911 \\
9 & 6 & 0 & 0.823574 & 1.220987 & -1.082613 \\
10 & 1 & 0 & 0.437241 & 0.722861 & -1.981417 \\
11 & 6 & 0 & 1.947987 & 2.055126 & -1.185954 \\
12 & 6 & 0 & 2.437417 & 2.676065 & -0.031442 \\
13 & 1 & 0 & 3.313122 & 3.332587 & -0.112534 \\
14 & 6 & 0 & 1.847146 & 2.481423 & 1.221113 \\
15 & 1 & 0 & 2.230070 & 2.963393 & 2.126051 \\
16 & 6 & 0 & 0.758318 & 1.633203 & 1.275672 \\
17 & 6 & 0 & -0.274464 & -1.473359 & 0.512056 \\
18 & 6 & 0 & 0.980869 & -1.607327 & 1.316420 \\
19 & 1 & 0 & 0.908595 & -1.592212 & 2.413277
\end{tabular}




$\begin{array}{lllrrr}20 & 6 & 0 & 2.202868 & -1.626083 & 0.717965 \\ 21 & 6 & 0 & 2.320467 & -1.738199 & -0.697993 \\ 22 & 6 & 0 & 1.162323 & -1.961432 & -1.464090 \\ 23 & 6 & 0 & -0.099361 & -1.903084 & -0.921581 \\ 24 & 6 & 0 & -2.214809 & 0.204699 & 0.041284 \\ 25 & 6 & 0 & -3.269282 & -0.585913 & 0.519789 \\ 26 & 1 & 0 & -3.098362 & -1.275913 & 1.353119 \\ 27 & 6 & 0 & -4.539651 & -0.491642 & -0.040042 \\ 28 & 1 & 0 & -5.348518 & -1.122519 & 0.346572 \\ 29 & 6 & 0 & -4.786538 & 0.401000 & -1.082585 \\ 30 & 1 & 0 & -5.786301 & 0.470178 & -1.526592 \\ 31 & 6 & 0 & -3.755342 & 1.216028 & -1.541456 \\ 32 & 1 & 0 & -3.939729 & 1.938274 & -2.345321 \\ 33 & 6 & 0 & -2.482665 & 1.122690 & -0.979585 \\ 34 & 1 & 0 & -1.686042 & 1.779043 & -1.346191 \\ 35 & 6 & 0 & 2.632866 & 2.215847 & -2.510575 \\ 36 & 1 & 0 & 3.154598 & 1.280655 & -2.796456 \\ 37 & 1 & 0 & 3.385482 & 3.023890 & -2.489378 \\ 38 & 1 & 0 & 1.913718 & 2.442988 & -3.319663 \\ 39 & 1 & 0 & 1.301331 & -2.214327 & -2.523675 \\ 40 & 6 & 0 & -1.301833 & -2.262023 & -1.734000 \\ 41 & 1 & 0 & -1.930758 & -1.385930 & -1.993897 \\ 42 & 1 & 0 & -1.006931 & -2.754258 & -2.678536 \\ 43 & 1 & 0 & -1.967285 & -2.954009 & -1.179357 \\ 44 & 8 & 0 & 0.102030 & 1.282068 & 2.430249 \\ 45 & 1 & 0 & -1.081147 & -2.074746 & 0.981651 \\ ---------------------------------------------------------------------------------\end{array}$

